\title{
REAL-TIME AUTONOMOUS OBSTACLE AVOIDANCE FOR UNMANNED AERIAL VEHICLES
}

\author{
by \\ Min Prasad Adhikari \\ Master of Applied Science, Ryerson University, (2015) \\ Bachelor of Engineering, Nanjing University of Aeronautics and Astronautics, (2012)
}

\author{
A dissertation \\ presented to Ryerson University \\ in partial fulfillment of the \\ requirements for the degree of \\ Doctor of Philosophy \\ in the program of \\ Aerospace Engineering
}

Toronto, Ontario, Canada, 2020

(C)Min Prasad Adhikari, 2020 


\section{AUTHOR'S DECLARATION FOR ELECTRONIC SUBMISSION OF A DISSERTATION}

I hereby declare that I am the sole author of this dissertation. This is a true copy of the dissertation, including any required final revisions, as accepted by my examiners.

I authorize Ryerson University to lend this dissertation to other institutions or individuals for the purpose of scholarly research.

I further authorize Ryerson University to reproduce this dissertation by photocopying or by other means, in total or in part, at the request of other institutions or individuals for the purpose of scholarly research.

I understand that my dissertation may be made electronically available to the public. 


\title{
REAL-TIME AUTONOMOUS OBSTACLE AVOIDANCE FOR UNMANNED AERIAL VEHICLES
}

\author{
Min Prasad Adhikari \\ Doctor of Philosophy, Aerospace Engineering, Ryerson University, Toronto (2020)
}

\begin{abstract}
In this dissertation, methods for real-time trajectory generation and autonomous obstacle avoidance for fixed-wing and quad-rotor unmanned aerial vehicles (UAV) are studied. A key challenge for such trajectory generation is the high computation time required to plan a new path to safely maneuver around obstacles instantaneously. Therefore, methods for rapid generation of obstacle avoidance trajectory are explored. The high computation time is a result of the computationally intensive algorithms used to generate trajectories for real-time object avoidance. Recent studies have shown that custom solvers have been developed that are able to solve the problem with a lower computation time however these designs are limited to small sized problems or are proprietary. Additionally, for a swarm problem, which is an area of high interest, as the number of agents increases the problem size increases and in turn creates further computational challenges. A solution to these challenges will allow for UAVs to be used in autonomous missions robust to environmental uncertainties.

In this study, a trajectory generation problem posed as an optimal control problem is solved using a sequential convex programming approach; a nonlinear programming algorithm, for which custom solver is used. First, a method for feasible trajectory generation for fast-paced obstacle-rich environments is presented for the case of fixed-wing UAVs. Next, a problem of trajectory generation for fixed-wing and quad-rotor UAVs is defined such that starting from an initial state a UAV moves forward along the direction of flight while avoiding obstacles and remaining close to a reference path. The problem is solved
\end{abstract}


within the framework of finite-horizon model predictive control. Finally, the problem of trajectory generation is extended to a swarm of quad-rotors where each UAV in a swarm has a reference path to fly along. Utilizing a centralized approach, a swarm scenario with moving targets is studied in two different cases in an attempt to lower the solution time; the first, solve the entire swarm problem at once, and the second, solve iteratively for a UAV in the swarm while considering trajectories of other UAVs as fixed.

Results show that a feasible trajectory for a fixed-wing UAV can be obtained within tens of milliseconds. Moreover, the obtained feasible trajectories can be used as initial guesses to the optimal solvers to speed up the solution of optimal trajectories. The methods explored demonstrated the ability for rapid feasible trajectory generation allowing for safe obstacle avoidance, which may be used in the case an optimal trajectory solution is not available. A comparative study between a dynamic and a kinematic model shows that the dynamic model provides better trajectories including aggressive trajectories around obstacles compared to the kinematic counterpart for fixed-wing UAVs, despite having approximately the same computational demands. Whereas, for the case of quad-rotor UAVs, the kinematic model takes almost half the solution time than with a reduced dynamic model, despite having approximately the similar range of values for the cost function. When extended to a swarm, solving the problem for each UAV is four to seven times computationally cheaper than solving the swarm as a whole. With the improved computation time for trajectory generation for a swarm of quad-rotors using centralized approach, the problem is now reasonably scalable, which opens up the possibility to increase the number of agents in a swarm using high-end computing machines for real-time applications. Overall, a custom solver jointly with a sequential convex programming approach solves an optimization problem in a low computation time. 


\section{ACKNOWLEDGEMENTS}

First of all, I would like to thank my thesis committee members, who managed their time during summer to view my dissertation draft and provide valuable suggestions which have improved the quality of this dissertation.

I am in debt to my supervisor Prof. Anton H. J. de Ruiter, who gave me an opportunity to pursue a doctoral program at Ryerson University. Without his support, the trajectory to pursue the dream would have been very different, or maybe a dream of a distant future. His guidance, support, and encouragement throughout the period is and will be admired. Moreover, it would have been impossible to prepare this thesis without his regular guidance.

I am grateful to my parents, and sisters for always supporting me to pursue my dream. Moreover, it would have been difficult without the patience of my wife, Sangita. Her encouragement and support all the time is a motivating factor. Also, my gratitude towards Deepak and Saru for their warm support.

And, it was always great to be around friends like Afshin, Alex, Amar, Dante, Emily, Frank, Kaustav, Micheal, Mike, Sobhan, Vasanth, Venky and more. 


\section{DEDICATION}

Dedicated to my parents, Mom and Dad. 


\section{TABLE OF CONTENTS}

Declaration .......................... ii

Abstract ............................... iii

Acknowledgements .....................

Dedication .............................. vi

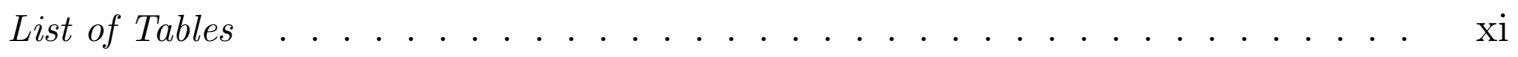

List of Figures . . . . . . . . . . . . . . . . . xiii

List of Appendices . . . . . . . . . . . . . . . . . . xvii

1 Introduction $\quad 1$

1.1 Literature Review . . . . . . . . . . . . . . . . . . . . . . . . 4

1.1.1 Reactive Obstacle Avoidance ................ . 4

1.1.2 Real-Time Trajectory Generation for UAVs . . . . . . . . . 10

1.2 Research Objectives. . . . . . . . . . . . . . . . . . . . . . . 14

1.2.1 Rapid Feasible Trajectory Generation for Collision Avoidance in Fixed-Wing UAVs . . . . . . . . . . . . . . . . . . . . 14

1.2.2 Real-Time Trajectory Generation for UAVs . . . . . . . . . . . 14

1.2.3 Real-Time Trajectory Generation for a Swarm of Quad-rotors . . 14

1.3 Dissertation Overview . . . . . . . . . . . . . . . . . 15

1.4 Dissertation Contributions . . . . . . . . . . . . . . . . . 20

2 Customization of Solver $\quad 22$

2.1 Introduction . . . . . . . . . . . . . . . . . . . . . 22

2.2 Sequential Convex Programming . . . . . . . . . . . . . . . . . . 24

2.2.1 Infeasible Start Newton Method . . . . . . . . . . . . . . 26

2.3 Customization of Solver . . . . . . . . . . . . . . . . . . . . . . . 29

2.4 Example of Customization . . . . . . . . . . . . . . . . . . 38 
2.4.1 Matrix-Vector Multiplication . . . . . . . . . . . . . . . . 39

2.4 .2 Custom KKT solver . . . . . . . . . . . . . . . . 40

2.5 Summary . . . . . . . . . . . . . . . . . . . . . 50

3 Rapid Feasible Trajectory Generation for Collision Avoidance in Fixedwing UAVs 51

3.1 Introduction . . . . . . . . . . . . . . . . . . . . 52

3.2 Problem Formulation . . . . . . . . . . . . . . . . . . . . . . 54

3.2 .1 Cost Function . . . . . . . . . . . . . . . . . 55

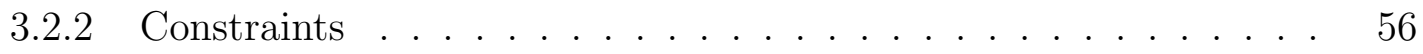

3.2 .3 Problem Definition . . . . . . . . . . . . . 57

3.2.4 Simplification of the System Dynamic Equation . . . . . . . 58

3.3 An Approach for a Feasible Trajectory Generation . . . . . . . . . . . 63

3.3 .1 Cost Function . . . . . . . . . . . . . . . 65

3.3 .2 Constraints . . . . . . . . . . . . . . . 66

3.3 .3 Problem Definition . . . . . . . . . . . . . . 68

3.4 Numerical Example . . . . . . . . . . . . . . . . . . . . . . . . 70

3.4 .1 Problem Scenario . . . . . . . . . . . . . . . . . 72

3.4 .2 Monte-Carlo Test . . . . . . . . . . . . . . . . . . . 76

3.4.3 Comparison Between Original and Simplified Problem . . . . . . . 83

3.4.4 Comparison with DIDO, ECOS and IPOPT . . . . . . . 83

3.4.5 Example: A Stationary and a Moving Obstacle . . . . . . . . 86

3.4.6 Example: Stationary Obstacles at the Centre of a Reference Path 96

3.5 Summary . . . . . . . . . . . . . . . . . . 106

4 Real-Time Trajectory Generation for Fixed-Wing UAVs 107

4.1 Introduction . . . . . . . . . . . . . . . . . . . . . . 108

4.2 Problem Formulation . . . . . . . . . . . . . . . . . . . . . . . 113

4.2.1 Dynamic Model for a Fixed-Wing UAV . . . . . . . . . . . . . 113

4.2.2 Kinematic Model for a Fixed-Wing UAV . . . . . . . . . . . . . 114

4.2 .3 Problem Definition . . . . . . . . . . . . . . . . . 116

4.2 .4 Problem Summary . . . . . . . . . . . . . . . . . . . . . 119

4.3 Finite Horizon Model Predictive Control Problem . . . . . . . . . . . . 120 
4.4 Numerical Examples . . . . . . . . . . . . . . . . . . . . . . . . . . . 124

4.4 .1 Scenario with Two Obstacles . . . . . . . . . . . . . . . . 124

4.4.2 Study on the Choice of Time Step, $\Delta t$ for Trajectory Generation . 134

4.4 .3 Monte-Carlo Test . . . . . . . . . . . . . . . . . 138

4.5 Summary . . . . . . . . . . . . . . . . . . . . . . 141

$5 \quad$ Real-Time Trajectory Generation for Quad-rotor UAVs 143

5.1 Introduction . . . . . . . . . . . . . . . . . . . . . . . . 144

5.2 Problem Formulation . . . . . . . . . . . . . . . . . . . . . . 145

5.2 .1 Quad-rotor System Equations . . . . . . . . . . . . . 145

5.2 .2 Problem Definition . . . . . . . . . . . . . . . 151

$5.3 \quad$ Numerical Examples . . . . . . . . . . . . . . . . . . . . . . 152

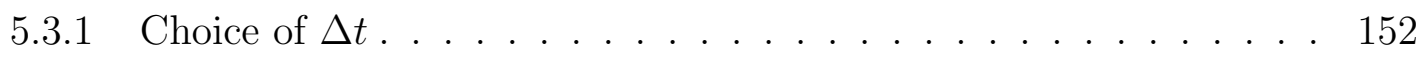

$5.3 .2 \quad$ Scenario with Obstacles . . . . . . . . . . . . 157

5.3 .3 Monte-Carlo Test . . . . . . . . . . . . . . . . . . . . . . . 162

5.4 Summary . . . . . . . . . . . . . . . . . . . . . 165

6 Quad-rotor Swarm Application 166

6.1 Introduction . . . . . . . . . . . . . . . . . . . 167

6.2 Problem Size for a Swarm . . . . . . . . . . . . . . . . 169

6.2.1 Problem Size Comparison ． . . . . . . . . . . . . 170

6.3 Swarm Problem with Reference Paths . . . . . . . . . . . . . . . . 173

6.3 .1 Problem Definition . . . . . . . . . . . . . . 174

6.3 .2 Numerical Example . . . . . . . . . . . . . . . . . . . . 175

6.4 Swarm Problem with Moving Targets . . . . . . . . . . . . . . . 180

6.4 .1 Problem Definition . . . . . . . . . . . . . . . 181

$6.4 .2 \quad$ Numerical Example . . . . . . . . . . . . . . . . . . . . 182

6.5 Iterative Trajectory Generation for a Swarm with Moving Targets . . . 186

6.5.1 Problem Definition . . . . . . . . . . . . . . 187

$6.5 .2 \quad$ Numerical Example . . . . . . . . . . . . . . . . . . . . . 188

6.5.3 Monte-Carlo Test . . . . . . . . . . . . . . . . . . . . . 193

6.5.4 Scalability of the Swarm Problem _. . . . . . . . . . . 193

6.6 Summary . . . . . . . . . . . . . . . . . . . 196 
7 Conclusion and Future Works 197

7.1 Conclusion . . . . . . . . . . . . . . . . . 197

7.2 Future Works . . . . . . . . . . . . . . . . 199

$\begin{array}{ll}\text { Appendices } & 200\end{array}$

References $\quad 234$ 


\section{List of Tables}

2.1 File sizes for the two approaches . . . . . . . . . . . . . . . . . . 48

3.1 Physical and aerodynamic features of the Ascent UAV [1]. . . . . . . 71

3.2 State constraints for the Ascent UAV . . . . . . . . . . . . . 71

3.3 Control constraints for the Ascent UAV . . . . . . . . . . . . . . . 72

3.4 Control constraints (3.24) for the simplified model (3.23) . . . . . . . 72

3.5 Parameter value for the problem $(3.40) \ldots \ldots \ldots \ldots$

3.6 Parameter value for the problem $(3.7) \ldots \ldots \ldots \ldots$

3.7 Comparison of computation time between the solvers . . . . . . . . 85

3.8 Iterations and solution time for feasible trajectory $\ldots \ldots \ldots \ldots$

4.1 Wind field for simulation . . . . . . . . . . . . . . . . . 125

4.2 Bounds on states and control variables for dynamic model. . . . . . . . . 125

4.3 Bounds on states and control variables for kinematic model. . . . . . . . 127

4.4 Penalty parameters to the cost function $(4.14) \ldots \ldots \ldots \ldots \ldots$

4.5 Comparison of the custom solver (CS) with IPOPT and ECOS: For trajectory with Kinematic model. . . . . . . . . . . . . . . . . . . . 133

4.6 Comparison of the custom solver (CS) with IPOPT and ECOS: For trajectory with Dynamic model . . . . . . . . . . . . . . . . 133

5.1 Problem parameters . . . . . . . . . . . . . . . . . . . . 153

5.2 Comparison of the custom solver (CS) with IPOPT and ECOS: For trajectory with Kinematic model. . . . . . . . . . . . . . . . . . 162

5.3 Comparison of the custom solver (CS) with IPOPT and ECOS: For trajectory with Dynamic model . . . . . . . . . . . . . . . 163

6.1 Size of the swarm problem using the UAV model (5.3) . . . . . . . . . 172 
6.2 Size of the swarm problem using the UAV model (5.4) . . . . . . . . . . . 172

6.3 Starting points and desired paths for quad-rotors . . . . . . . . . 176

6.4 Comparison of custom solver (CS) with IPOPT and ECOS. . . . . . . . 180

6.5 Starting points for quad-rotors (units in meter) . . . . . . . . . . . 183

6.6 Comparison of custom solver (CS) with IPOPT and ECOS. . . . . . . . 192

6.7 Swarm problems with their average solution time . . . . . . . . . 196 


\section{List of Figures}

1.1 A representation of a scenario for the GeoSurv II UAV. . . . . . . . . . . 3

1.2 Trajectory generation with RRT and Dual Tree RRT [2] . . . . . . . . . 6

1.3 Numerical approach for trajectory generation . . . . . . . . . . . . . 16

1.4 Use of custom solver in SCP method . . . . . . . . . . . . . . . 17

1.5 Control architecture for trajectory generation . . . . . . . . . . . 19

2.1 Sequential convex programming approach . . . . . . . . . . . . 28

2.2 Custom solver generation . . . . . . . . . . . . . . . . . . 37

2.3 General solver and custom solver comparison . . . . . . . . . . . . . . . . 49

$3.1 N$ discrete points in 3D space. . . . . . . . . . . . . . 64

3.2 Obstacle configuration for Monte-Carlo simulation . . . . . . . . . . . . 74

3.3 Cost comparison . . . . . . . . . . . . . . . . . . . . 79

3.4 Computation time comparison . . . . . . . . . . . . . . . . . 79

3.5 Iteration comparison . . . . . . . . . . . . . . . . 80

3.6 Cost comparison . . . . . . . . . . . . . . . . . 80

3.7 Computation time comparison . . . . . . . . . . . . . . . 81

3.8 Iteration comparison . . . . . . . . . . . . . . . . . 81

3.9 Computation time comparison between $C t_{1}$ and $C t_{2}+C t_{I G}$. . . . . . 82

3.10 Iteration comparison between $I t_{1}$ and $I t_{2}+I t_{I G} \ldots \ldots$

3.11 Cost comparison between the original and the simplified problem with Monte-Carlo test. . . . . . . . . . . . . . . . 83

3.12 Cost comparison between DIDO, IPOPT, ECOS and CS to solve simplified problem with feasible initial guess. . . . . . . . . . . . . . . 84

3.13 Computation time comparison between DIDO, IPOPT, ECOS and CS to solve simplified problem with feasible initial guess. . . . . . . . . . . 
3.14 Obstacle configuration for the example . . . . . . . . . . . . . 86

3.15 Acceleration $a \ldots \ldots \ldots \ldots$. . . . . . . . . . . . . . 87

3.16 Rate of change of flight path angle $\omega_{\gamma} \ldots \ldots$. . . . . . . . . 88

3.17 Rate of change of heading angle $\omega_{\psi} \ldots \ldots$. . . . . . . . . . . . . 88

3.18 Thrust $T$. . . . . . . . . . . . . . . . . . . . . . . . . . . 89

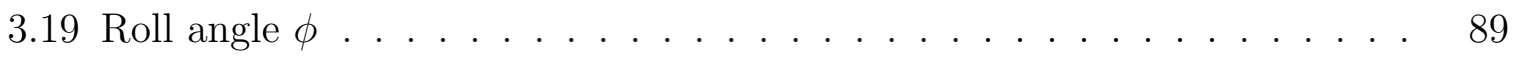

3.20 Angle of attack $\alpha \ldots \ldots$. . . . . . . . . . . . . . . . . . 90

3.21 Feasibility test of dynamic controls . . . . . . . . . . . . . . . . . . 91

$3.22 \mathrm{XZ}$ view of the obstacle avoidance scenario . . . . . . . . . . . . . . 91

$3.23 \mathrm{XY}$ view of the obstacle avoidance scenario . . . . . . . . . . . . . . 92

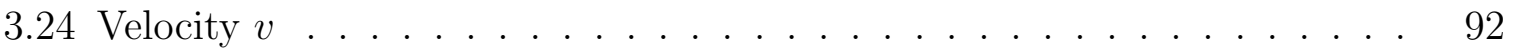

3.25 Heading angle $\psi \ldots \ldots$. . . . . . . . . . . . . . . . . . . . . . . . 93

3.26 Flight path angle $\gamma \ldots \ldots$. . . . . . . . . . . . . . . . . 93

3.27 Distance to moving obstacle . . . . . . . . . . . . . . . . . . . . . 94

3.28 The progress in the cost for 15 SCP iterations . . . . . . . . . . . . . . . 94

3.29 The computation time for $15 \mathrm{SCP}$ iterations . . . . . . . . . . . . . . 95

3.30 Solution time for Raspberry PI 3B (15 SCP iterations) . . . . . . . . . . 95

3.31 Stationary obstacles with various initial guesses, 3D view . . . . . . . . . 99

3.32 Stationary obstacles with various initial guesses, XY view . . . . . . . . . 99

3.33 Stationary obstacles with various initial guesses, XZ view . . . . . . . . . 100

3.34 Stationary obstacles with various initial guesses, YZ view . . . . . . . . . 101

3.35 Feasible trajectories for the initial guesses, 3D view . . . . . . . . . . . 102

3.36 Feasible trajectories for the initial guesses, XY view . . . . . . . . . . . . 103

3.37 The progress of slack (s) and eigenvalue for the Initial Guess 1 . . . . . . 104

3.38 The progress of slack (s) and eigenvalue for the Initial Guess 2 . . . . . . 104

3.39 The progress of slack (s) and eigenvalue for the Initial Guess 3 . . . . . . 105

3.40 The progress of slack (s) and eigenvalue for the Initial Guess 4 . . . . . . 105

4.1 The value of $g(t)$ for obstacle robustness factor . . . . . . . . . . . . . 118

4.2 Example scenario. . . . . . . . . . . . . . . . . . . . . . 125

4.3 Flight trajectory of the Fixed-Wing UAV, 3D view . . . . . . . . . . . . 128

4.4 Flight trajectory of the Fixed-Wing UAV, XY view . . . . . . . . . . . . 128 
4.5 Flight trajectory of the Fixed-Wing UAV; zoomed near the stationary obstacle. . . . . . . . . . . . . . . . . . . 129

4.6 Flight trajectory of the Fixed-Wing UAV; zoomed at the constant wind region. . . . . . . . . . . . . . . . . . . . 130

4.7 Computation time per instance (0.2 second) for online simulation using an Intel processor. . . . . . . . . . . . . . . . . . . . . . . 131

4.8 Computation time per instance for online simulation using an ARM processor. . . . . . . . . . . . . . . . . . . . . 132

4.9 Trajectories using the kinematic model for different value of $\Delta t \quad \ldots \ldots$

4.10 Trajectories using the dynamic model for different value of $\Delta t \ldots \ldots$. . 137

4.11 The total flight time per scenario . . . . . . . . . . . . . . . . . 140

4.12 Computation time per instant for all scenarios . . . . . . . . . . . . . 141

4.13 Total value of square of devation . . . . . . . . . . . . . . . . . 142

5.1 Quad-rotor model . . . . . . . . . . . . . . . . . . . . . . . . . . . 145

5.2 Trajectories using the dynamic model for different values of $\Delta t \ldots \ldots 155$

5.3 Trajectories using the kinematic model for different values of $\Delta t \ldots \ldots$

5.4 Trajectory comparison, 3D view . . . . . . . . . . . . . . . 158

5.5 Trajectory comparison, XY view . . . . . . . . . . . . . . . . . . 159

5.6 Trajectory comparison around moving obstacles, XZ view . . . . . . . . . 159

5.7 Computation time comparison . . . . . . . . . . . . . . . . . 160

5.8 Computation time comparison in Raspberry PI 3B . . . . . . . . . . 161

5.9 Thrust required for trajectories . . . . . . . . . . . . . . . . . 161

5.10 Computation time per instant for all scenarios . . . . . . . . . . . 164

5.11 Total value of square of deviation . . . . . . . . . . . . . . . 165

6.1 Problem size comparison between the kinematic and the dynamic model 173

6.2 Swarm problem $2 \ldots \ldots \ldots \ldots \ldots$

6.3 3D view for the swarm example . . . . . . . . . . . . . . 176

$6.4 \mathrm{XY}$ view for the swarm example . . . . . . . . . . . . . . . 177

$6.5 \mathrm{XZ}$ view for the swarm example . . . . . . . . . . . . . 177

6.6 The swarm scenario at $t=25$ seconds . . . . . . . . . . . . . 178

6.7 The swarm scenario at $t=26$ seconds . . . . . . . . . . . . . . . . 178 
6.8 XY view of the swarm scenario at $t=39.9$ seconds $\ldots \ldots \ldots$. . . . 178

$6.9 \mathrm{XZ}$ view of the swarm scenario at $t=39.9$ seconds . . . . . . . . . . 179

6.10 Solution time for the swarm problem at time $t$. . . . . . . . . . . 180

6.11 Swarm problem with moving targets . . . . . . . . . . . . . 181

$6.123 \mathrm{D}$ view of the swarm with moving targets . . . . . . . . . . . . 183

$6.13 \mathrm{XY}$ view of the swarm with moving targets . . . . . . . . . . . . . . . 184

$6.14 \mathrm{XZ}$ view of the swarm with moving targets . . . . . . . . . . . . . . . 184

6.15 The swarm at flight time $t=53.6$ seconds . . . . . . . . . . . . 185

6.16 The swarm at flight time $t=73.6$ seconds . . . . . . . . . . . . . 185

6.17 The swarm at flight time $t=93.6$ seconds $\ldots \ldots \ldots$. . . . . . 186

6.18 Solution time for a MPC time instant for the swarm problem with moving targets . . . . . . . . . . . . . . . . . 187

$6.193 \mathrm{D}$ view for the swarm with moving targets . . . . . . . . . . . . . 189

$6.203 \mathrm{D}$ view for the swarm with moving targets . . . . . . . . . . . . . . . 190

$6.21 \mathrm{XZ}$ view for the swarm with moving targets . . . . . . . . . . . . . . 190

6.22 Distance bewteen UAVs . . . . . . . . . . . . . . . . . . . . . 191

6.23 Solution time comparison between swarm problems with moving targets . 192

6.24 Computation time per instances a UAV . . . . . . . . . . . . . . . . 194

6.25 Computation time per instances for UAVs . . . . . . . . . . . . . . 194

6.26 Computation time for various swarm size . . . . . . . . . . . . 195 


\section{LIST OF APPENDICES}

1 Customization of Solver, Examples 201

1.1 Custom Factorization Example: First Approach . . . . . . . . . . . . . . 201

1.2 Custom Solve Example: First Approach . . . . . . . . . . . . . . . . . . 202

1.3 Custom Factorization Example: Second Approach . . . . . . . . . . . . . 203

1.4 Custom Solve Example: Second Approach . . . . . . . . . . . . . . . . . 205

2 Bounds on the Kinematic Constraints 207

3 A Brief Note on the Convexification 215

4 Quad-rotor Characteristics and Problems Parameters 218

4.1 Quad-rotor Characteristics . . . . . . . . . . . . . 218

4.2 Bounds on Optimization Variables . . . . . . . . . . . . . . . . . . 218

4.3 Bounds on Optimization Variables for Monte-Carlo Test . . . . . . . . . 219

4.4 Problem Scenario One . . . . . . . . . . . . . . . . . . . . 220

4.5 Problem Scenario Two . . . . . . . . . . . . . . . . . . . . 220 


\section{Chapter 1}

\section{Introduction}

In this dissertation, methods for real-time trajectory generation and autonomous obstacle avoidance for Unmanned Aerial Vehicles (UAVs) are studied. UAVs, also known as drones are flying machines without a human pilot on-board. Their increased ability to efficiently perform challenging missions compared to manned flight missions, and their relatively lower expense have gained them popularity. Moreover, the advancement in computational and electronics technologies are replacing manned flight missions with UAVs, and are opening new areas of application. Their use in disaster relief, conservation, national parks, scientific research, are some examples. Among such examples, the use of UAVs for performing geophysical surveys is one. Surveying poorly known large areas is a timeconsuming and expensive task for manned missions. Instead, a UAV flying over the terrain can do such a surveying mission efficiently. Thus, this dissertation supports such missions, to enhance the use of a UAV by increasing its autonomous ${ }^{1}$ capability. Increase in the autonomy of a UAV system for such a surveying mission not only reduces human effort but also improves team productivity.

The original motivation for the study was to support the GeoSurv II project, with the overall objective to stay close to a reference path for the purpose of developing geomagnetic survey maps. The GeoSurv II project was a part of a collaboration between Sander Geophysics Limited (SGL), Carleton University and Ryerson University. The aim of the project was to perform high-resolution geophysical surveys by flying a UAV at low altitude (usually 50 meter above ground level) in rural areas. The GeoSurv II UAV is

\footnotetext{
${ }^{1}$ Autonomy is namely the ability of a machine to perform without human intervention.
} 
a fixed-wing twin-boom pusher aircraft with a wingspan of 4.9 meters, which, without autonomy is remote controlled. The UAV with a radio-control has a limitation on the flight range, which is constrained by the range of the radio signals. This limits the range of such a mission if it is to be remotely controlled from a manned ground station. In addition to that, successful avoidance of an obstacle by a UAV depends on the ground operator's ability to detect and maneuver around an obstacle. The complexity increases further when a UAV has to fly fifty meters above ground level, where it is challenged to avoid obstacles such as power lines and towers, tall trees or even unknown flying objects. However, the scenario for the UAV is not expected to be an urban cluttered environment. Our aim was to aid the UAV project by adding a level of autonomy such that the UAV could do the geophysical survey missions autonomously. This could potentially extend the range of mission that the UAV could perform, and also could relieve effort on the human ground operator. To accomplish this, the UAV needs to have rapid sense and avoid capabilities. Such detection and avoidance system must perform successful maneuvers in the presence of stationary ${ }^{2}$ and moving obstacles ${ }^{3}$. Although autonomy requires both the detection and avoidance capabilities, this dissertation supports only obstacle avoidance (OA). Therefore, in this disseration, we do not consider the problem of obstacle detection. This was the task for another team to perform obstacle detection by using stereoscopic vision. Then, using some technique of feature extraction the team would provide approximate location, size and shape of obstacles. A summary of obstacle detection proposed for the UAV project is outlined in [3]. Since we do not deal with the detection part, in terms of an environment modeling we do not have a full map of the environment beforehand. However, we do need to assume something about the location and shape of each obstacle in order to generate a trajectory. Therefore, obstacles in this disseration are modeled by primitive shapes (sphere, ellipsoid, cuboid). The similar modelling of obstacles for urban environment have been used in [4]. Moreover, map gets updated on real time as more data is collected (the work of detection team) thus making the environment modeling more accurate. For the purpose of a geomagnetic survey of a poorly known terrain the area is traced out by connecting lines as a reference path but without a goal point. Figure 1.1 shows a model representation where black dashed lines shows a reference path without a goal point, and obstacles such as trees and power towers

\footnotetext{
2 Those obstacles that are fixed. Examples, trees, power lines, and towers, tall terrain or small hill

${ }^{3}$ Objects that are moving, for example, flying vehicles, birds obstacles scenarios.
} 
are modeled as circular (in 2D) structures (for rural environment). These obstacle types are well modelled using the obstacle shapes considered in this dissertation.

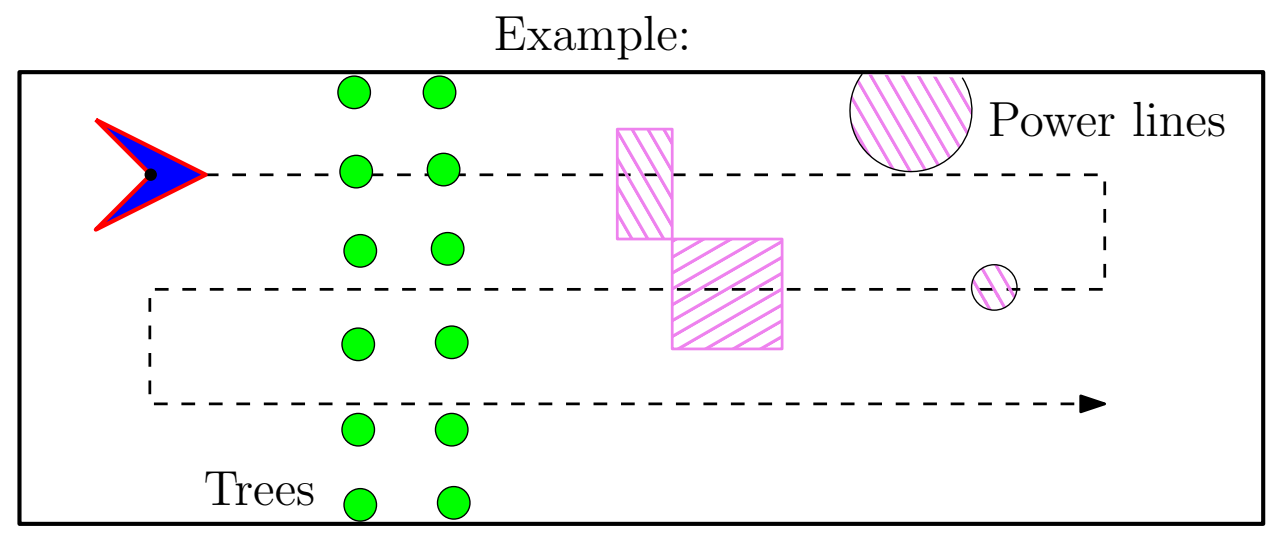

Figure 1.1: A representation of a scenario for the GeoSurv II UAV.

Thus, this dissertation studies the complementary technology that could have make the GeoSurv II UAV autonomous, such that the UAV could do autonomous avoidance in a poorly known terrain in the presence of stationary and moving obstacles. However, with the cancellation of GeoSurv II project the studies in this dissertation are more generalized to cover general UAVs to the application we consider, by also considering quad-rotors and its swarms.

In order to develop the capability of autonomous trajectory generation for obstacle avoidance onboard a UAV, the main constraint is the computation time taken by an onboard computer for a feasible (a flyable safe trajectory) trajectory generation. Typically on-board flight computers have much less computational capability compared to ground based computers, due to both size (payload capacity) and cost restrictions. However, the aim is the development of methods that can be used for real-time trajectory generation for UAVs with a wide range in size. And, methods that can employ algorithms with low memory footprints as an embedded system onboard a UAV with minimum hardware resources would be an asset. Thus, this dissertation studies methods that make rapid trajectory generation for UAVs possible. 


\subsection{Literature Review}

Trajectory generation for UAVs is a broad topic. Therefore, to limit ourselves to trajectory generation and autonomous obstacle avoidance methods that could be implemented in real-time, literature survey is presented in two different subsections. The first is on Reactive Obstacle Avoidance (ROA), and the second is on real-time trajectory generation for UAVs.

\subsubsection{Reactive Obstacle Avoidance}

Autonomous systems are becoming an integral part of our daily life equipment, making a complex system look simple and easy to use. The idea of autonomy might have existed for more than a century, but it started taking preliminary shape around the 1920s. Around the 1980s some university labs started showing the viability of autonomous system through experimental projects [5] and primitive examples [6]. These autonomous activities were limited only to ground vehicles under laboratory environments. Later around the 1990s when the technology started maturing, a need for higher autonomy urged around the globe. This became evident motivated not only engineers but also mathematicians, physicists, and even politicians. Recently, advanced autonomous UAVs may be within reach of modern technology. As an example, Reference [7] has shown that a quadcopter UAV can avoid obstacles autonomously. However, in the case of a fixedwing UAV, the problem is more complicated as a fixed-wing UAV must fly above its stall speed and has turning constraints. The nature of dynamics being different between fixed-wing and rotary wing UAVs, an approach used for an autonomous mission for a quad-rotor UAV cannot be directly applied to a fixed-wing UAV. However, the converse may be true. In the literature, algorithms for the avoidance of obstacles have been further divided into two categories, 1) trajectory planning or the global path planning and 2) local path planning or the Reactive Obstacle Avoidance (ROA).

When a complete map of the environment is taken into account for the path planning of a vehicle before the start of the mission, such a method is called trajectory planning. It is also known as global path planning. Whereas, when a map of the environment is updated during the flight with new obstacle information as it becomes available, such kind of method is known as the reactive obstacle avoidance or local path planning. For 
the global path-planning, there are many luxuries, such as computation resources and time are not of significant concern, since trajectories may be generated off-line prior to flight. On the other hand, reactive obstacle avoidance is performed onboard in realtime, with generally limited computational resources. In the literature, there are many algorithms for path planning, but few of them qualify for reactive obstacle avoidance. In the following, the literature survey summarizes advancements in the trajectory generation method used for reactive obstacle avoidance for UAVs.

\section{Rapidly-Exploring Random Tree}

Rapidly-Exploring Random Tree (RRT) is a probabilistic sampling-based search algorithm that can find a possible path in cluttered environments with search metric such as safety [8]. In this method, a 'tree' like structure is generated at the initial point which forms the root nodes. Then, the 'tree' is expanded adding nodes until it reaches the goal position. After that, a particular search algorithm is used to find a path between the initial and the target point that satisfies a given metric. Amin et. al. have used this method for global path planning in the presence of obstacles [9]. Various path planning applications using this approach have been shown in reference [10]. Report by Kuffner. et. al. have used an advanced version of this method, the Dual-RRT method, where a trajectory is integrated from both the start and the goal point together simultaneously at each iteration to reduce the computation time [11]. Frazzoli et al. have shown in the simulation results, that the RRT can be used to solve a difficult ground-based trajectory planning problem including the problem with moving obstacles. Figure 1.2 from [2] shows a comparison between RRT and Dual-RRT, where sub-figures (a) and (b) are the results of RRT at 1 and 30 seconds respectively, while sub-figures (c) and (d) are the results of Dual-RRT at 1 and 30 seconds respectively. In the figures, blue lines represent the workspace, state trees are represented by green lines, and the red line represent the selected trajectory. The bottleneck of the method is the computation time, which is in tens of seconds, and it increases exponentially as the number of obstacle increases (in particular with moving obstacles) [12]. 


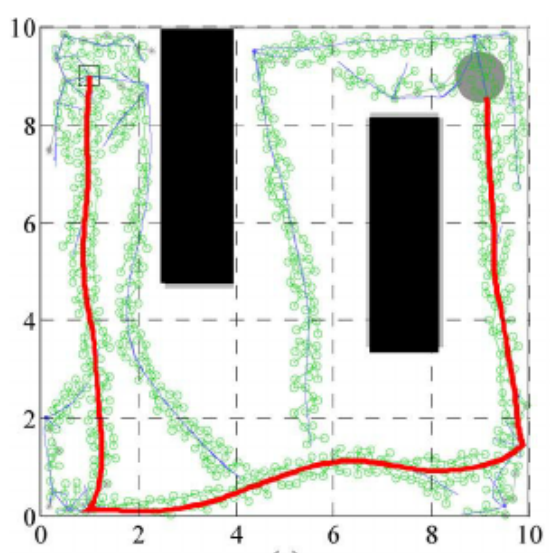

(a)

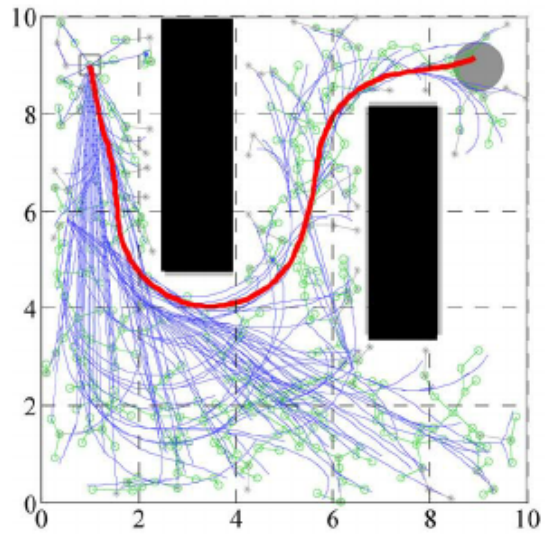

(c)

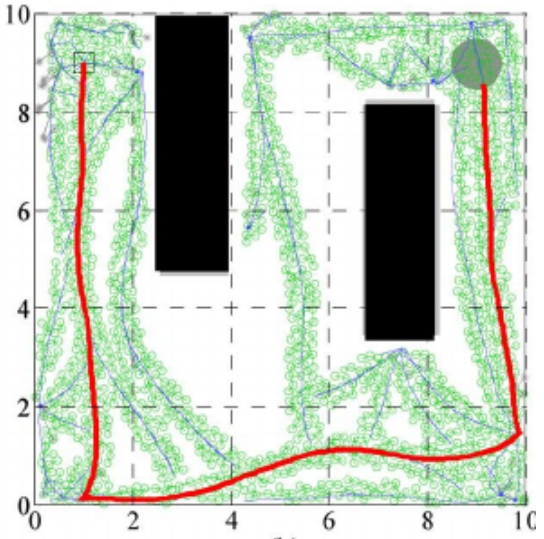

(b)

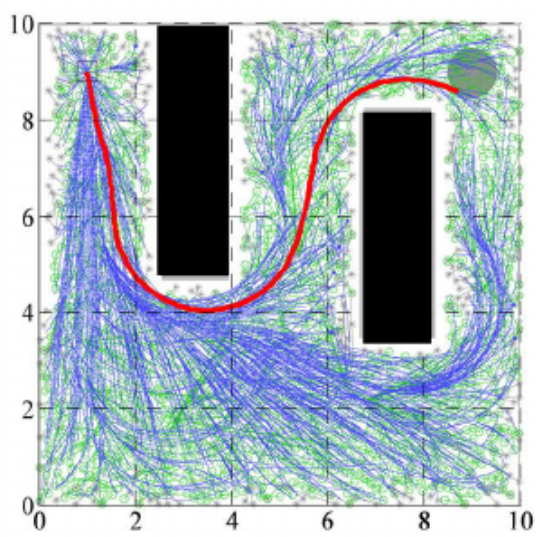

(d)

Figure 1.2: Trajectory generation with RRT and Dual Tree RRT [2]

\section{Artificial Potential Field Method}

The Artificial Potential Field method uses the concept of potential field, where the target is assigned an attractive potential, and an obstacle is assigned a repulsive potential. By this approach, an object is attracted towards the target location while being repelled by obstacles. The artificial driving force which acts as a control input to an object or a vehicle is the resultant of potential virtual forces, where these forces are computed as the negative gradient of the potential field. This approach is suitable for a real-time path generation where obstacles can be avoided easily by assigning repulsive potentials; nevertheless, it has several drawbacks. One of the drawbacks is, a UAV can get stuck in a local minimum point of the potential field. Other approaches have to be implemented to recover from such a situation. Other drawbacks presented in the Reference [13] are 
due to the location of potential fields. An example of this phenomenon when a target is under the influence of a high potential field obstacle, and a UAV is moving in a straight line towards the target such that the target is in between the UAV and the obstacle. In this case, the object moving towards the target also moves towards the obstacle. Thus, the UAV may not reach the target due to the repulsive force of the obstacle, even if there is no obstacle in-between the UAV and the target. Another example is when an obstacle is in-between a UAV and a target such that they are moving at the same speed in the straight line. Although they are moving, their relative position and velocity remain constant (virtually stationary). Thus, the constant relative position and the constant relative velocity would result in the constant potential force while in motion, which will never let the UAV reach the target. The Reference [13] also suggests some possible solutions for these types of situations.

One of the improvements done to the traditional potential field method to the problem of collision/obstacle avoidance is the introduction of gyroscopic control forces [14]. Details on theoretical development can be found in Reference [14], and citaions therein. This variant has advantages like, there are no local minima, and obstacles are avoided locally if they appear to be within the safety shell defined. Some practical aspects of potential fields are studied in [15], and it has shown a successful result for the case of Unmanned Ground Vehicles (UGVs) [16]. However, the method alone do not yield trajectories that are optimal with respect to some specified cost function. The potential field concept has also been adopted in optimal control problem for trajectory optimization.

\section{Optimal Control Methods}

The optimal control method could pose the problem of trajectory generation and autonomous obstacle avoidance for UAVs, however, there are various methods to solve an optimal control problem. Indirect methods being a branch of approaches to solving an optimal control problem, it is challenging to solve them by transforming into a two-point boundary value problem. However, the method had led a basis for trajectory optimization [17]. Some indirect methods (such as the indirect shooting method, near extreme method and other numerical methods) that are developed after the 1980s resulted in high sensitivity to initial starting points for the evaluation of a trajectory generation problem ([18], Chapter 7), and challenging for solving complex constraints conditions [19]. The 
next branch of approach is the direct iterative methods that involve defining a trajectory generation problem as an optimal control problem, performing some sort of discretization, and solving it as a parameter optimization problem. The direct methods have the advantage that they can accommodate a complex constraint condition into a single problem formulation. In general, there are two classes of approach for solving optimal control problems using gradient-based direct optimization techniques, such as [20] and [21]. The first is a discretize-linearize approach and the second is the linearize-discretize approach.

In the discretize-linearize approach, the problem is first discretized and then solved using a nonlinear programming (NLP) technique. For example, one can use a Pseudospectral method for the discretization of the problem [20], and then solve the discretized problem using a nonlinear programming algorithm such as SNOPT [22], where SNOPT linearizes the problem internally during the iterative procedure. By the use of pseudospectral discretization method, an optimal control problem goes through various intermediate steps such as the selection of a proper discretization scheme which determines the convergence and accuracy of the solution, state and control approximation, Ordinary Differential Equation (ODE) approximation, approximation of the cost function and finally, the scaling and balancing of the problem (details can be found in [23]). Then, the problem is finally posed as a discrete nonlinear parameter optimization problem and solved using a nonlinear programming solvers [22, 24]. Using this method, one has to solve a finite-dimensional nonlinear problem. Among various pseudospectral schemes, the Legendre-Gauss-Lobatto discretization has been used in conjunction with an optimal control method for real-time trajectory generation. Details on the discretization scheme can be found in the paper by Ross et al. [20]. Results from the literature show that the scheme has been used in simulations of real-time trajectory generation for Unmanned Ground Vehicle (UGV), Unmanned Aerial Vehicle (UAV) and also re-entry vehicles [25, 26, 27]. Reference [28] highlights the method's potential for real-time trajectory planning. The key to real-time trajectory generation or reactive obstacle avoidance using this method lies in solving the problem efficiently in terms of computation time.

The second approach, the linearize-discretize method involves a linearization of a problem (generally quadratic cost and linear constraints) and then discretization at specified intervals. For example, one can convexify the problem and then discretize with a fixed time-step. An example of such an approach is the Model Predictive Control [21] method. Model Predictive Control (MPC) is a control strategy where future controls 
for the plant model are predicted based on a certain performance index. At practical application, all the model predictive controls (MPC) are discrete-time. In discrete model predictive control (MPC), at each sampling instant, a finite horizon optimal control problem is solved based on the present plant state and control constraints which yield an optimal control sequence. Among the optimal control sequences, the first of its control sequence is applied to the plant model and latter are taken as predictions for future controls. Practically, the method is used as a repetitive decision-making process utilizing finite horizon optimal control. A model predictive control (MPC) law is feedback implicitly evaluated at the current state; however, prediction and optimized predictions are open-loop. Details on the model predictive control (MPC) and their practical applications can be found in references $[29,30]$. In this approach, one has to solve a discrete problem, usually a Quadratic Programming (QP) problem. Thus, this approach for trajectory generation allows using the method of convex optimization which is more mature than non-convex methods. One such method that utilizes a convex approximation of a nonlinear programming (NLP) problem is the Sequential Convex Programming (SCP) ([31], Page 123). In the sequential convex programming (SCP) method, a general nonlinear programming (NLP) problem is approximated by a convex problem around a given point to find a locally optimal solution, and the process of convexification and solving the convex problem continues until the solution to the original nonlinear programming problem (NLP) is reached. The reference [32] outlines a trust region based sequential convex programming (SCP) method to solve a nonlinear programming (NLP) problem, where the convergence guarantee of the sequential convex programming (SCP) with a trust region radius starting from a remote point to an optimal solution is available in the reference. However, by choosing the second approach, one no longer solves the originally posed optimal control problem, but rather uses it within a model predictive control (MPC) framework. This is particularly beneficial for real-time trajectory generation, where trajectories need to be updated once new environmental information is available. Using model predictive conrol (MPC), Shim et al. in [7] present result of their work on real-time path planning for the avoidance of obstacles in rotorcraft UAVs, and in [33] Shim et al. used model predictive control (MPC) where head-on collision with a hostile obstacle is avoided successfully in a helicopter. It has been shown that the method can account for moving obstacles including collision avoidance among UAVs. Likewise, Wang et al. in [34] used model predictive control (MPC) framework for cooperative UAV 
(helicopter) formation flying with obstacle/collision avoidance and suggested previewing mechanism of receding horizon control to be ideal for such application.

\subsubsection{Real-Time Trajectory Generation for UAVs}

UAVs can carry out various life-threatening tasks without significant risks. Their possibilities for further use have prompted extensive research in both academia and industry. Remote data collection is a challenging application of UAVs, as it requires a remote control, particularly in an obstacle-rich environment. When the UAV should be controlled from a longer distance owing to poor visibility, signal quality and lag control response issues are raised, as longer distance implies longer signal transfer ([35], Chapter 2). Lag could lead to a potential failure of a mission or could at least damage a UAV. This problem would be significantly mitigated if a UAV could operate autonomously, with a flight computer on board that observes a situation, understands the problem, decides a solution, and acts accordingly (this is also called observe-orient-decide-act in [36]) in real-time.

For real-time generation of a trajectory onboard a UAV, one major challenge is the computation capability onboard. This is mainly due to the use of nonlinear programming (NLP) algorithms to solve the trajectory generation problem, as nonlinear programming (NLP) methods are known for their time-consuming iterations which are not favorable for real-time application. In particular, for the case of fixed-wing UAVs, which have non-zero minimum speed, rapid calculation of controls and updating of environment (obstacles and terrain) information are required to fly safely. However, real-time trajectory generation onboard fixed-wing UAVs is still a topic of ongoing research [37]. While for the case of quad-rotors, although they have the luxury to stop mid-air and take time for a decision, they benefit from rapid trajectory generation.

Although the computational complexity of trajectory generation for a UAV depends on the number of constraints imposed, an optimal solution is more expensive in terms of computation amount and time compared to a feasible solution for the same problem. A feasible trajectory generation from an initial point to the target point so that obstacles are avoided, state and control constraints are satisfied, and mission objectives are met represents one aspect of the task, whereas searching for an optimal trajectory is another. Even though the optimal trajectory is obviously superior in terms of quality, a feasible 
trajectory that can be rapidly obtained is preferable so that a UAV may safely fly in an obstacle-rich environment, especially for fixed-wing UAVs. Furthermore, for the problem of obstacle avoidance when a UAV should fly above its stall velocity, a feasible trajectory can ensure safer flight among obstacles compared to the optimal trajectory, which requires considerable computation time.

Regarding the computability of a trajectory generation problem, a UAV model chosen may impact the solution time as well. For example, reference [38] used a six degrees of freedom UAV model for a study of path-planning, which complicates the problem compared to with a simpler kinematic model. However, the use of a dynamic model for a UAVs trajectory generation may allow for full utilization of UAV's capabilities, rather than conservatively specifying these constraints up-front as constants and then utilizing a simpler kinematic model. A dynamic model is particularly important when obstacles suddenly appear in front of a UAV, and an aggressive evasive maneuver is necessary, especially for fixed-wing UAVs which fly above stall speed with constrained turning radii. However, for the case of a quad-rotor UAV, a kinematic model might work well due to the flexibility in flight constraints of the UAV. In the literature there are various references that have used a dynamic model for quad-rotors in their study of trajectory generation like [39] and [40], but a simpler model for a quad-rotor UAV as similar to the one used in reference [41] and [42] may be sufficient for collision-free trajectory generation. Although, the use of a high fidelity model for a quad-rotor UAV may produce a steady trajectory; but, it may be computationally intensive when it comes to trajectory generation for a swarm of quad-rotors due to the increment in the number of variables with the UAVs. Regarding the computational aspects of swarm problems, references like $[43,44,45]$, have presented their work towards a trajectory generation for a swarm application and the associated computational time. Moreover, the outdoor swarm implementations currently seen in the literature implement trajectories that are generated offline $[46,47,44,48]$. With respect to the implementation of a generated trajectory, a common practice is to pass a trajectory to an autopilot which tracks the trajectory using a low-level flight controller [49].

With regards to advances in the continuous time planning or polynomial trajectory generation, the following papers present the work of various authors. For the study of trajectory planning Bry et al. in [50] have presented their work in which they used the combination of path search algorithm with polynomial trajectory optimization. In the 
approach, a new waypoint is added if a trajectory is found to intersect an obstacle, after which the polynominal trajectory optimization approach has to be repeated with the added waypoints but without directly incorporating dynamic or kinematic constraints. Finally, from the optimized polynomial trajectory dynamically feasible trajectories are computed from starting to goal point. Although the paper demonstrates an experimental results the trajectory utilized is computed offline with the global map. Likewise, Nuske et al. in [51] present motion planning for a quad-rotor UAV for navigating rivers using SPARTAN; a tool that utilizes an $A^{*}$ like approach for path searching in the presence of obstacles. Although the solution time shown in the paper is low, the paper addresses different application than ours. Moreover, the paper does not disclose the UAV model used. Similarly, Loianno et al. in [52] study planning for aggressive flight for a quadrotor. The paper presents the joint study of estimation, control and planning for quadrotor UAVs where the continuous time planning has be used for agressive maneuvers. The paper uses a dynamic model for the UAV for which only feasible trajectories are sought. Although experimental results have been shown, details on solution time are still undisclosed.

\section{An Extension to quad-rotor Swarm Problems}

At the present context when it comes to swarm application, the flexibility in speed and the complexity in a trajectory offered by a quad-rotor outweighs a high endurance mission from a fixed-wing UAV. Thus, this dissertation supports swarm application for quadrotors. In the literature, regarding the different aspects of quad-rotors swarm problems, Honig et al. used an approach that consists of three steps (road map generation, discrete planning, and continuous refinement to smooth trajectory) for trajectory planning for quad-rotor swarm [46], and it has no concern with real-time computation as it is a trajectory planning. Kushleyev et al. studied the trajectory generation and collision avoidance in a swarm with agile micro quad-rotors [47]. In [44], Luis et al. studied the offline trajectory generation method for a swarm using Distributed Model Predictive Control and argues the advantage against sequential convex programming (SCP) method in terms of computation time. In [53], Campobasso studied a trajectory generation for a swarm with a leader-follower approach. Similarly, the reference [54] studied a swarm of quad-rotor UAVs for collision-avoidance, path planning and challenges with 
communication using hardware currently available in the market. The reference [48] presented a study on communication method adopted for control of a swarm of quad-rotor for light shows. Similarly, in [55] Preiss et al. presented a study on control of a swarm of quad-rotor UAVs in a laboratory environment. Authors in [56] studied a distributed auction assignment method for control for a large swarm of agents. In addition, references $[56,48]$ have studied an approach for a swarm trajectory generation in which trajectory is generated for an agent at a time in a swarm. In [41], the authors have used the simplified model of quad-rotor for trajectory generation. The result from the reference shows that the computation time is low, which is also a motivating factor for us to study a simplified model for quad-rotors.

\section{Customization of solver}

A sequential convex programming (SCP) algorithm that solves for a convex approximation to a nonlinear programming (NLP) problem requires a repetitive solving of a standard quadratic programming (QP) problem, which may be computationally intensive. Thus, there are various work in the literature that aimed at lowering the solution time to such quadratic programming (QP) problems. One of the latest work done towards that is the development of custom code generation platform called CVXGEN [57] which generates custom solver to a standard quadratic programming problem. However, it is very inconvenient to define problems of any sparsity pattern using the platform. Moreover, the CVXGEN is targeted at solving problems of small size (problems with altogether 2000 coefficients in the constraints and objective) within milliseconds, and cannot guarantee a successful code generation once the number of non-zeros in a KarushKuhn-Tucker (KKT) matrix goes beyond 4000 [58]. Besides that, there are other numerically stable solvers for embedded application such as, ECOS [59] designed for secondorder cone problems (SOCP), FORCES targeted at solving quadratically constrained quadratic programming (QCQP) problems, quadratic programming (QP) problems, and linear programming (LPs) problem [60], and FiOrdOs designed at supporting general

convex problems [61], and some of them are proprietary. However, when it comes to a problem specific customization, there is room for improvement on speed, accuracy and memory allocation by customization at the code level. 


\section{$1.2 \quad$ Research Objectives}

Real-time generation of avoidance trajectory onboard UAVs provides an edge for UAVs autonomous missions, with various unforeseen economical advantages. Therefore, the following problem statements with their respective research objectives are studied in this dissertation.

\subsubsection{Rapid Feasible Trajectory Generation for Collision Avoid- ance in Fixed-Wing UAVs}

The objective here is to study methods that can be used for rapid generation of feasible trajectory with endpoints in an obstacle-rich environment for the case of fixed-wing UAVs. Since a trajectory generation problem for fixed-wing UAVs posed as an optimal control problem can be transformed as a nonlinear programming problem (NLP), our objective is to study methods for rapid solvability of the problem.

\subsubsection{Real-Time Trajectory Generation for UAVs}

The real-time trajectory generation problem without definite endpoint for fixed-wing and quad-rotor UAVs are studied under this heading. Thus, the objective is to study methods that look into the immediate window for trajectory generation and solves the problem for real-time application.

\subsubsection{Real-Time Trajectory Generation for a Swarm of Quad- rotors}

As the methods are studied for real-time trajectory generation for UAVs in the objective 1.2.2, the aim here is to build upon the successful approach from the previous objective for swarm application of quad-rotors. Since scalability of a swarm problem relies on the computational capability, our objective is to study approaches to further decrease the solution time to a swarm problem. 


\subsection{Dissertation Overview}

Given a trajectory generation problem with boundary conditions, kinematic or dynamic constraints, and obstacles avoidance conditions, the real-time solvability of the problem mainly depends on the computer processing power and the solution method used. Since, small UAV applications are constrained from the availability of powerful computers, our problem of real-time trajectory generation hangs around an algorithm for the solution of the problem. The trajectory generation problem can be posed as an optimal control problem, which can be transformed to a nonlinear programming (NLP) problem, and methods exist to solve it by approximating the problem with a sequence of quadratic programming (QP) problems, also known as sequential quadratic programming (SQP). Figure 1.3 shows a flowchart where a trajectory generation problem can be posed as an optimal control problem with cost function and constraints. Then, the optimal control problem can be transformed to a nonlinear programming (NLP) problem by using appropriate discretization methods. Although, methods such as sequential quadratic programming (SQP) exist for solving a nonlinear programming problem, motivated by the rapid solution of convex problems, convex approximations of nonlinear programming problems will be solved. Thus, sequential convex programming (SCP) will be utilized in this disseration.

Utilizing the sequential convex programming (SCP) approach, a nonlinear programming (NLP) problem will be approximated by a quadratic programming problem, which will then be solved using a quadratic programming algorithm. As shown in the flowchart in Figure 1.4, if the stopping criteria is not reached yet, then the nonlinear problem will be approximated at the solution of previous quadratic problem and then solved again using the quadratic programming algorithm. However, motivated by the improvement in solution time as seen in the literature, a program is developed in Chapter 2 that generates customized quadratic programming algorithms to speed up the computation time. Thus, generated customized quadratic programming algoritm is termed as custom solver, and it has a least memory footprint to solve a convex quadratic problem. The solver generation program can generate custom solvers for small to relatively large sized quadratic programming (QP) problems which will be applicable in the subsequent chapters in this dissertation.

Since small UAVs are constrained to the availability of small computers as an onboard 


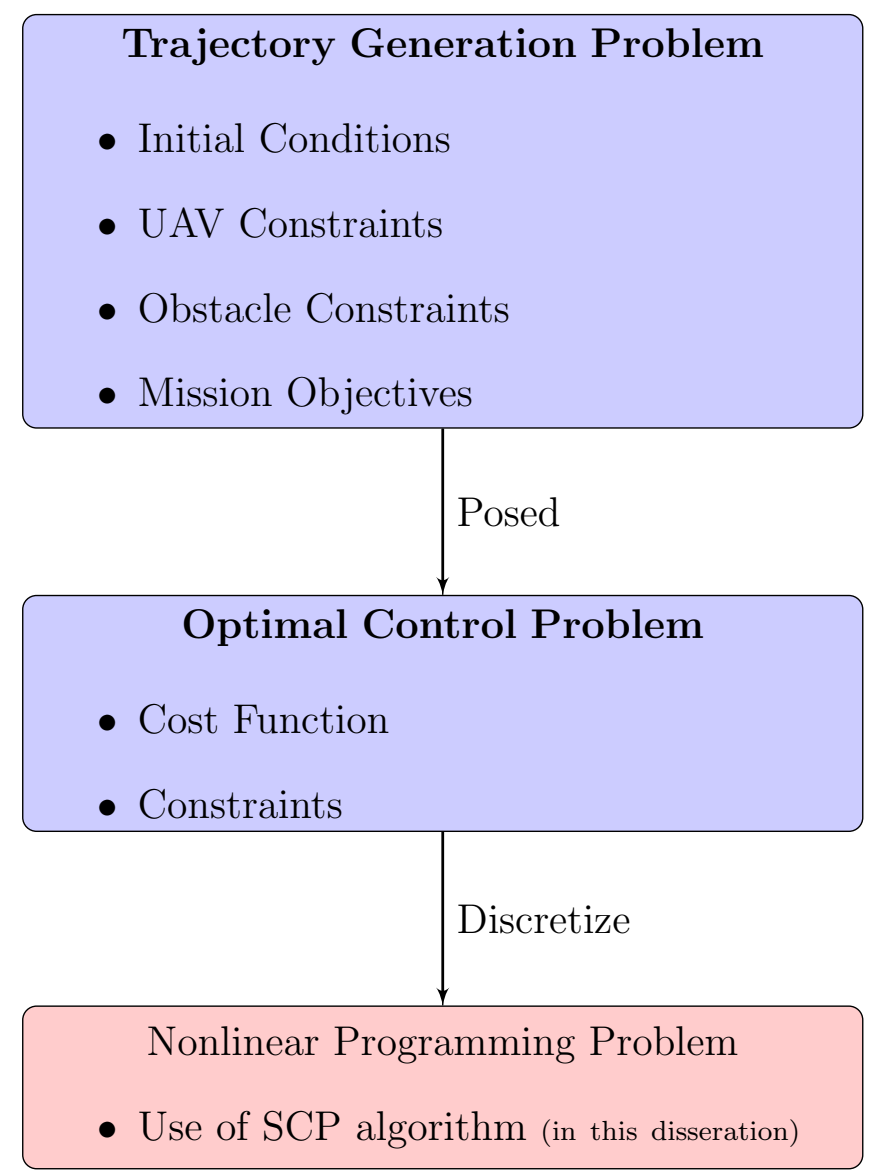

Figure 1.3: Numerical approach for trajectory generation

computer, the custom solver generated can be placed as an embedded application of the real-time trajectory generation system. The custom solvers developed are geared towards solution of convex quadratic programming (QP) problems with the Hessian of the overall cost function to the trajectory generation problem being positive semi-definite. However, a trajectory generation problem that includes obstacle avoidance, and robustness factor in the cost function may have indefinite overall cost Hessian. Therefore, to deal with the indefinite Hessian, an approach is summarized as similar to an Infeasible Start Newton method in Chapter 2.

As a trajectory generation problem with boundary conditions, kinematic or dynamic constraints, obstacle avoidance condition, that includes control saturation and a certain performance index can be transformed to a nonlinear programming (NLP) problem, its solution time cannot be approximately pre-determined. Thus, this uncertainty in the 


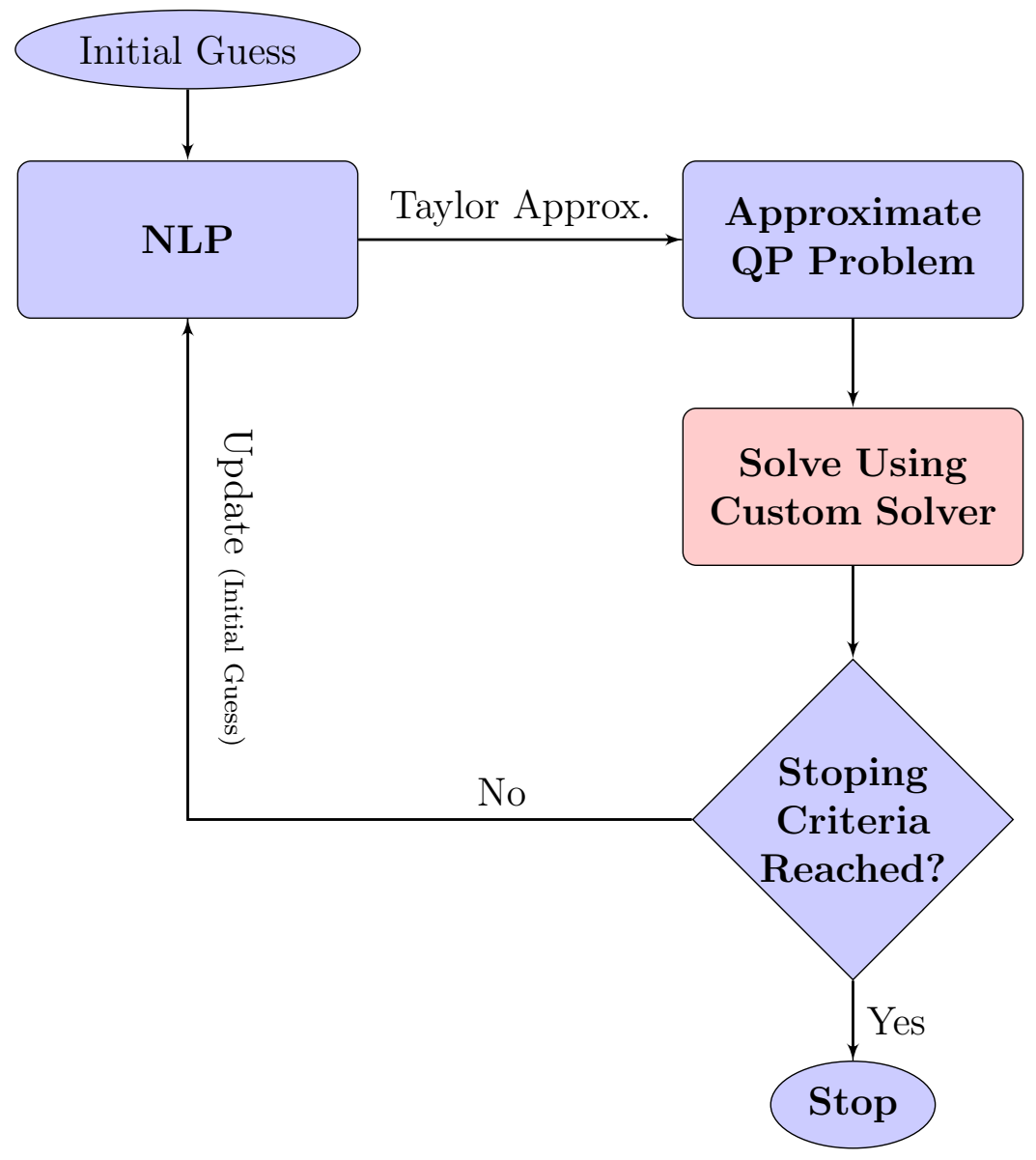

Figure 1.4: Use of custom solver in SCP method

solution time will impact the fixed-wing UAVs more than quad-rotors UAVs. Unlike a quad-rotor, which can hover at a point during a flight until it gets the update in desired trajectory, a fixed-wing UAV has to fly above its stall speed and cannot wait until the solution arrives. Therefore, a method for rapid feasible trajectory generation for fixed-wing UAVs is presented in Chapter 3 utilizing the sequential convex programming approach, and its purpose is to ensure feasible trajectory, so that a fixed-wing UAV may fly safely among obstacles even though an optimal solution, which is generally computation-intensive, may not be available. Moreover, a feasible trajectory can also be used as an initial guess to obtain an optimal solution with significantly reduced computation time. Although a rapid solution generation will aid quad-rotor UAVs for a quick update in an environment for obstacle avoidance, it is straight forward to generate 
a feasible trajectory for quad-rotors due to its flexibility in speed and turning radius.

Ideally, it is desirable to solve the trajectory generation problem posed as an optimal control problem. As stated before, using a general nonlinear programming (NLP) method to solve the problem is computationally costly; therefore an approach of using the sequential convex programming (SCP) method together with a custom solver was chosen. Regarding the implementation of the generated trajectory on a UAV, it is not necessary to have the entire trajectory available, instead just a portion of the trajectory over an immediate short window is enough. Besides, the appearance of any new obstacles during flight necessitates re-solving the trajectory generation problem. Therefore, a Finite Horizon Model Predictive Control approach is used, with the same constraints and the same cost function to the original problem, to solve the real-time trajectory generation problem for fixed-wing UAVs in Chapter 4 and for quad-rotor UAVs in Chapter 5. Moreover, these chapters examine the use of a kinematic and a dynamic model for both the UAVs for real-time application. Figure 1.5 shows a flowchart for trajectory generation where trajectory generation using model predictive control sends the generated trajectory to an autopilot, the autopilot sends controls to a UAV, and the UAV updates states and obstacles information to trajectory generation block at every sampling time. Note that this dissertation only deals with the trajectory generation aspect, not the full control system including autopilot. Thus Figure 1.5 is just to illustrate how the trajectory generation system studied in this dissertation would fit into an overall autonomous flight control system.

Moreover, regarding the UAVs application for autonomous obstacle avoidance, a high fidelity model which provides a more natural accommodation of the UAV constraints compared to a lower fidelity model, the ability to avoid collision improves. However, the use of a dynamic model for a UAV into trajectory generation complicates the solution procedure. Although a simpler formulation may serve the purpose of a feasibility study, it does not account for the dynamic behaviour of a UAV when an aggressive maneuver is required. Thus, an advantage of a kinematic model for trajectory generation is its simplicity for incorporation with collocation methods, while a disadvantage for a dynamic model may be a computational complexity. Hence, this dissertation presents a comparative study between a dynamic and its kinematic counterpart for real-time trajectory generation and autonomous obstacle avoidance for fixed-wing and quad-rotor UAVs.

Although fixed-wing UAVs are attractive for high endurance mission, their use in 


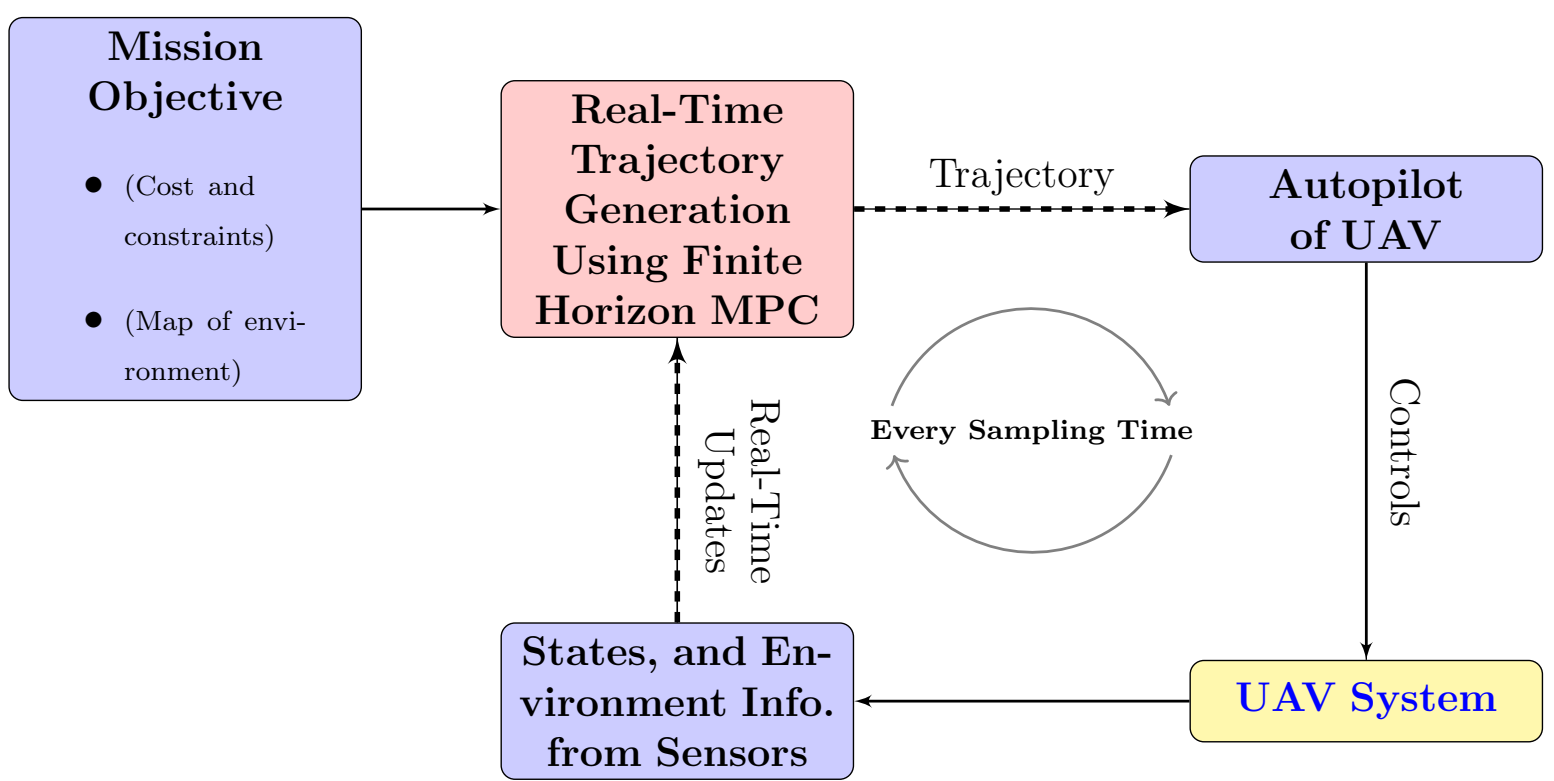

\section{UAV Systems: Fixed-Wing and Quad-rotor.}

Figure 1.5: Control architecture for trajectory generation

swarm application are less likely when compared with quad-rotor UAVs, due to the flexibility in speed and the complexity in a trajectory that quad-rotors could offer. Therefore in Chapter 6 the problem of real-time trajectory generation with an autonomous obstacle and collision avoidance for a swarm of quad-rotors is studied. In particular, a centralized approach is utilized, and dynamical, and control constraints are accommodated. The centralized approach has the benefit of utilizing all available information, in contrast to decentralized approaches. Therefore the problem has the potentiality to serve various important applications that demand reliability and safety in a swarm while addressing a quality task provided a central computer such as a ground station is available. However, computational complexity being one of the challenges that constrain the centralized approach for swarm scalability, the use of custom solver to lower the solution time for the swarm problem is presented. Besides, the Chapter includes a comparable study for a scenario between two different cases; the first, solve the swarm problem at once, and the second, solve for an agent in a swarm at a time.

Finally, Chapter 7 presents the conclusion of the dissertation and possible future 
works.

\subsection{Dissertation Contributions}

- A simplification approach for fixed-wing UAVs dynamics equation is introduced along with its control constraints to obtain a simpler kinematic equation for fixedwing UAVs that preserves the bounds on the original dynamic controls. Then, the controls of the original dynamic equation can be calculated from the controls of the simplified system equations. Thus utilizing the approach, a trajectory generation problem needs only four optimization parameter $(x, y, z, v)$ (which leads to a low solution time), compared to a trajectory generation problem (in $3 D$ space) which usually has its states and control inputs as optimization parameters, including $x, y, z$ points and angles as states, and as well as control inputs to the model.

- An optimal control formulation for trajectory generation for fixed-wing and quadrotor UAVs within a framework of Finite Horizon Model Predictive Control and in the presence of obstacles, with the goal of staying close to a desired flight path, is presented. A smooth penalty function associated with obstacle robustness function is introduced such that the penalty term only penalizes obstacles close to the desired flight path, and the obstacles far from the desired flight path have no influence over the generated trajectory.

- Using the proposed optimal control formulation implemented in a model predictive control framework, a comparative study between a kinematic and a dynamic model for rapid trajectory generation and autonomous obstacle avoidance for fixedwing UAVs are performed, where with similar computation time between the use of models for the UAV, the dynamic model shows better results in terms of avoiding obstacles and remaining close to the desired trajectory. The approach yields a computation time which is low enough for implementation, and at the same time does not require a trajectory library. And by contrast, for the case of quad-rotor on the same optimal control formulation implemented in the model predictive control framework, the comparative study shows that the kinematic model performs computationally better than the dynamic model over approximately the similar quality of trajectory. 
- The optimal control formulation within the model predictive control framework introduced is used to define quad-rotor swarm problems in which quad-rotors aim to reach moving targets, which in then solved in two ways, in an attempt to lower the solution time. In the first way, the optimal control problem within the model predictive control framework is solved by considering the entire swarm at once. In the second way, it is solved iteratively for a UAV while considering trajectories from other UAVs as fixed. A numerical example shows that the second approach is almost four to seven times computationally faster than solving the problem by the first approach using our custom solver. In addition, using the second approach, a swarm problem with $32 \mathrm{UAVs}$ is solvable in 194.24 milliseconds in a laptop computer with $1.8 \mathrm{GHz}$ processor (Intel Core i5) and 4 GB RAM (1600 MHz DDR3). 


\section{Chapter 2}

\section{Customization of Solver}

\section{$2.1 \quad$ Introduction}

In this chapter, a general trajectory generation problem is defined, and a custom solver is presented which will be used in subsequent chapters to solve this type of problem rapidly. Moreover, the customization approach is presented.

Let us define a model trajectory generation problem, where $X$ and $U$ represent state and control variables respectively, and let a system model be given by

$$
\dot{X}(\tau)=f(X(\tau), U(\tau))
$$

where $f$ is continuous and differentiable, and the bounds on state and control variables are given by $\underline{X}, \bar{X}$ and $\underline{U}, \bar{U}$, with under bar $(\cdot)$ and over bar $\overline{(\cdot)}$ representing the lower and the upper bounds respectively.

Let us consider a state-control constraint which can be described by

$$
g(X(\tau), U(\tau)) \leq 0
$$

where $g$ is continuous and differentiable. Furthermore, let us consider an initial and the possible goal point constraints by

$$
X\left(\tau_{0}\right)=X_{0}
$$


and

$$
X\left(\tau_{f}\right)=X_{f}
$$

where $\tau_{0}$, and $\tau_{f}$ represent the starting and final time respectively. Note that while the starting time is fixed, the final time is allowed to be free.

A performance index is considered to be minimized in the trajectory generation problem given by,

$$
J(X(\tau), U(\tau), \tau)=\int_{\tau_{0}}^{\tau_{f}} F(X(\tau), U(\tau)) d \tau
$$

where $F$ is continuous and at least twice differentiable. In addition $F$ can have a convex component related to the desired performance, and a possibly non-convex robustness function to assist with obstacle avoidance.

Now, a model trajectory generation problem as an optimal control problem is defined as follows, given an initial condition (2.3) and the final condition (2.4), find the controlstate pair $(X(\cdot), U(\cdot))$ that minimizes the cost $(2.5)$ subject to

$$
\text { System equation }(2.1)+
$$

Collision avoidance constraint $(2.2)+$

Initial condition (2.3), and the final condition (2.4)

$$
\begin{aligned}
& \underline{X} \leq X \leq \bar{X} \\
& \underline{U} \leq U \leq \bar{U}
\end{aligned}
$$

In this thesis, an optimal control problem as above is first transformed into nonlinear programming problem by performing some sort of discretization, which is then solved using a gradient-based nonlinear programming algorithm.

In general, nonlinear programming algorithms are computationally intensive, and the rate of convergence may be slow from a remote infeasible starting point to a feasible solution. Therefore, in this dissertation significant efforts have been made to lower the computational time by lowering the computational complexity and by using a nonlinear programming approach that solves an approximate convex problem to the original problem. By choosing a method that solves a convex problem to arrive at the solution of a nonlinear programming problem, a reliable convex algorithm that has been shown to provide rapid convergence within a small approximate number of iterations can be used. 
Moreover, the solution is always a global optimal to the convex sub-problem. One such method that utilizes a convex approximation of a nonlinear programming problem to arrive at a solution is the sequential convex programming (SCP) ([31], Page 123). In the sequential convex programming method, a general nonlinear programming problem is approximated by a convex problem around a given point to find a locally optimal solution, and the process of convexification and solving the convex problem continues until the solution to the original nonlinear problem is reached. In this dissertation the sequential convex programming method outlined in reference [32] is utilized, which provides a trust region based sequential convex programming to solve a nonlinear programming (NLP) problem (where the convergence guarantee of the sequential convex programming (SCP) approach with a trust region radius starting from a remote point to an optimal solution is available in [32]). Thus, in the remainder of this chapter, the method to transform a nonlinear programming (NLP) problem to a convex sub-problem in a trust region radius is described, and an approach to solve it using a convex solver (a standard quadratic programming (QP) solver) is shown.

\subsection{Sequential Convex Programming}

Sequential Convex Programming is an approach for solving a nonlinear programming (NLP) problem iteratively by approximating it with a sequence of convex sub-problems [31]. A type of convex programming also known as quadratic programming (QP) often solves a subproblem of the nonlinear programming (NLP) algorithm. Such nonlinear programming (NLP) algorithms that use a quadratic programming (QP) approach for a sequence of iterates are often called nonlinear programming (NLP) by sequential quadratic programming (SQP) [22]. An example is the trust-region-based interior-point method that uses sequential quadratic programming (SQP) by Byrd et al. [32], which is also used in this thesis. However, in this disseration the method is used within a convex frame work and it is called a sequential convex programming (SCP). Discretization of (2.6) results in an nonlinear programming (NLP) problem of the form: 


$$
\begin{array}{cc}
\text { Minimize } & f_{0}(x) \\
\text { Subject to: } & f_{i}(x) \leq 0 \\
& h_{j}(x)=0 \\
& i=1, \ldots, m \\
& j=1, \ldots, p
\end{array}
$$

where $x \in \mathbb{R}^{n}$. $f_{0}(x)$ and $f_{i}(x)$ can be non-convex, and $h_{j}(x)$ can be non-affine, with a convex component of $f_{0}(x)$ from a desired perfromance index and a possible non-convex component from robustness function for obstacle avoidance. In the case of non-convexity of the overall $f_{0}(x)$, the problem (2.7) is posed in a framework that resembles an infeasible start newton method summarized in Section 2.2.1. Introducing the slack variable $s$ associated with the inequality constraints, (2.7) becomes

$$
\begin{array}{cc}
\text { Minimize } & f_{0}(x) \\
\text { Subject to: } & f_{i}(x)+s_{i}=0 \\
& h_{j}(x)=0 \\
& s_{i} \geq 0 \\
& i=1, \ldots, m \\
& j=1, \ldots, p
\end{array}
$$

where $s_{i}$ is a non-negative slack variable associated with each inequality constraint. The problem (2.8) can now be approximated using an interior-point method as in [32]:

$$
\begin{array}{cc}
\text { Minimize } & f_{0}(x)-\mu \sum_{i=1}^{m} \ln \left(s_{i}\right) \\
\text { Subject to: } & f_{i}(x)+s_{i}=0 \\
& h_{j}(x)=0 \\
& s_{i}>0, \mu>0 \\
& i=1, \ldots, m \\
& j=1, \ldots, p
\end{array}
$$

where $\mu$ is a positive penalty term, and the slack variables $s_{i}(i=1, \ldots, m)$ are assumed to be positive. The nonlinear programming (NLP) problem (2.9) can be solved by using a quadratic programming (QP) approximation at each iteration. However, the convergence of the problem depends on the starting point [32]. Therefore, to ensure convergence from 
a remote starting point, and to handle the case of non-positive-definite Hessian of the quadratic approximation to the cost function of (2.8), Byrd et al. in [32] proposed a trust region for the quadratic programming (QP) approximation. Moreover, the completeness of the algorithm can be followed from [32].

Let $f(x, s)$ represent the cost function and $c(x, s)=0$ represent the equality constraints of problem (2.9). Let $w=(x, s)^{T}$; then [32] proposed solving a quadratic approximation to the problem (2.9) at a given point $\bar{w}$ within a trust region $\left(\|d\|_{T} \leq \triangle\right)$, where $d=\left(\begin{array}{c}d_{x} \\ d_{s}\end{array}\right)^{T}, d_{x}=x-\bar{x}$ and $d_{s}=s-\bar{s}$ are small increments in $x$ and $s$, respectively, $\triangle$ is a positive radius also known as the trust region radius, and $\|\cdot\|$ is a norm. In the present case, the convex approximation to the problem type (2.9) at $\bar{w}$ is solved, with the trust region radius in terms of the Euclidean norm, $\left(\|d\|_{2} \leq \triangle\right)$, as follows:

$$
\begin{array}{cc}
\text { Minimize } & \nabla f(\bar{w})^{T} d+\frac{1}{2} d^{T} \nabla^{2} f(\bar{w}) d \\
\text { Subject to: } & \nabla c(\bar{w})^{T} d+c(\bar{w})=0 \\
& \|d\|_{2} \leq \triangle
\end{array}
$$

where the quadratic cost and the constraints are the second- and the first-order Taylor approximations to the cost and the constraints from the problem type (2.9), respectively. The details on obtaining a suitable trust region radius $(\triangle)$ and its update can be found in [32].

\subsubsection{Infeasible Start Newton Method}

Although the use of a convex solver has been stated in the introduction of this chapter, our overall cost function such as in (4.14) may not be convex. Therefore, we may not be strictly solving a convex sub-problem always, which as shown in later chapters may be an issue near an obstacle boundaries. Besides, the starting point for the UAV may not satisfy the dynamics and obstacle constraints. Thus, to deal with the cases when the cost function is non-convex, a method that is similar to the infeasible start Newton method ([62], Chapter 10.3) is used to arrive at a feasible solution. Also, the infeasibility due to obstacles and dynamics constraints is not an issue for convergence, which will be apparent later during the solution procedure.

In order to find the solution to (2.8), the approach solves a quadratic programming 
(QP) approximation to (2.8) with a trust region radius. Since, the approach solves for a quadratic programming (QP) approximation, but not the convex approximation, there is a possibility that the Hessian of the overall cost function may turn be indefinte, and in that case an indefinite matrix factorization may be used to solve the quadratic programming (QP) problem. However, for the case of an indefinite Hessian the customization of the quadratic programming (QP) solver may not be possible, or at least we have no knowledge of such a custom quadratic programming (QP) solver that deals with the indefinite Hessian, as the pivoting required during matrix factorization may not be fixed. Therefore, to avoid such an indefinite Hessian case, the quadratic approximation to (2.11) is solved until the slack variable $(s)$ is zero.

$$
\begin{array}{cc}
\text { Minimize } & \overline{f_{0}}(x, s)+\zeta s^{2} \\
\text { Subject to: } \quad \epsilon_{s}+f_{i}(x)-s \leq 0 \\
\\
h_{j}(x)=0 \\
i=1, \ldots, m \\
j=1, \ldots, p
\end{array}
$$

where $\overline{f_{0}}(x, s)$ is continuous, twice differentiable and convex. The function $f_{0}(x)$ in $(2.7)$ is modified with the addition of slack variable to form $\overline{f_{0}}(x, s)$, which are convex around the region of interest. For the nature of problems considered in this thesis, $\overline{f_{0}}(x, s)$ represents a function which is made convex by the choice of slack variable $s$ such that a custom QP solver could be used. $\zeta>0$ is a penalty term associated with the slack variable, $s \geq 0, \epsilon_{s}>0$ is a constant used for efficient numerical solving. This approach of solving (2.11) instead of (2.7) in the case of an obstacle infeasibility also resembles the infeasible start Newton method. The advantage of solving (2.11) is, the overall cost Hessian to the problem can be made positive semidefinite (by choice of the slack variable). The reason to solve a convex problem is, the possibility of customization of a quadratic programming solver for the convex problem which will be apparent in the next section. The aim of solving (2.11) is to find a feasible starting point for (2.7) in the case of obstacle infeasibility at the initialization which is the main source of an indefinite Hessian for our trajectory generation problems. Since the overall cost Hessian to (2.11) for our trajectory generation problems goes very close to convex even inside an obstacle (due to the slack variable), it always converges to a locally optimal solution. Moreover, 
once (2.11) is solved, the overall cost Hessian to (2.7) becomes positive semidefinite as the obstacle constraint becomes feasible and the second order Taylor approximation to the robustness function becomes positive semidefinite outside an obstacle boundary, then the regular procedure of solving (2.8) to get an optimal solution can be followed. In the third chapter of this dissertation where a feasible trajectory generation problem is solved, an example case is solved where initialization was done exactly at the centre of obstacles. In the example, even if the problem was started with initial guesses within obstacles (initialization is given inside obstacles boundary), (2.11) solves for a feasible trajectory. Here, still a convex problem is solved. At precisely the center of an obstacle, the quadratic programming (QP) problem is not convex, but the eigenvalue of the Hessian is so small that it does not affect the solver $\left(-1.694 \times 10^{-10}\right)$.

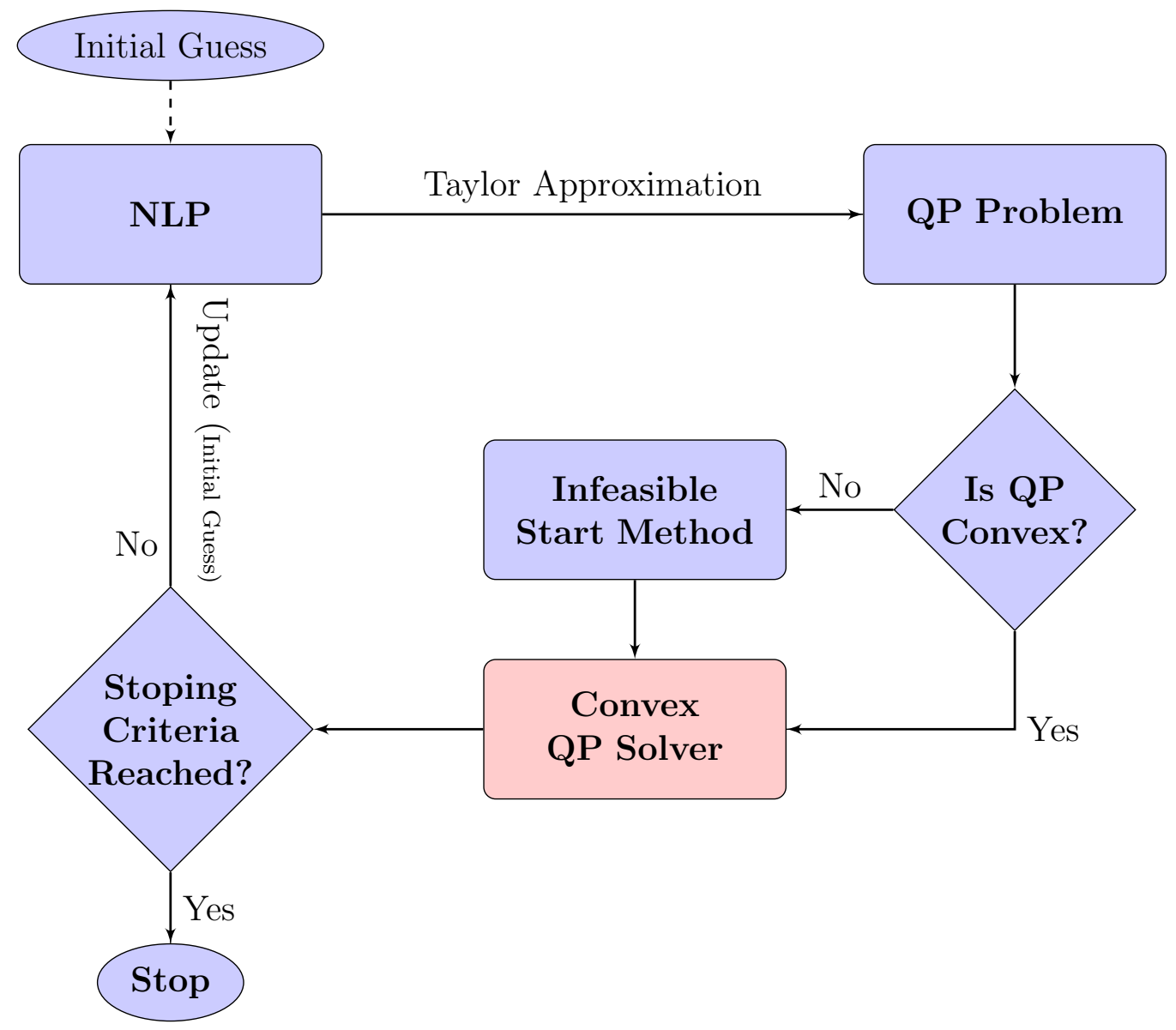

Figure 2.1: Sequential convex programming approach 
Figure 2.1 shows a flowchart for the sequential convex programming approach, where a nonlinear programming problem approximated by a quadratic problem, which is solved using a convex quadratic programming algorithm until a stopping criterion is reached. In case of non-convexity of the quadratic problem, the nonlinear problem utilizes an infeasible start Newton-like method which forges an approximate quadratic problem to a convex problem, and after this a convex quadratic programming algorithm is utilized as usual.

In the approach of sequential convex programming that we utilize, the first goal is to find a feasible solution. Once a feasible solution is found, then the solution progresses towards optimal value at each SCP iterate. Therefore, if the solver is interrupted before reaching the optimal value, a feasible solution should be expected. Moreover, to lower the computation time for the algorithm a custom solver is used.

\subsection{Customization of Solver}

In order to solve a standard quadratic programming (QP) problem, various gradientbased algorithms are available (for example, SQIC [63], QPIP [32], first-order method and Active-set method [64]). Moreover, there are some quadratic programming (QP) solvers readily available such as ECOS [59] designed for second-order cone problems (SOCP), FORCES targeted at solving Quadratically Constrained QP (QCQP) problems, quadratic programming (QP) problems, and Linear Programming (LPs) problems [60], and FiOrdOs designed at supporting general convex problems [61]. These solvers are numerically stable and are suitable for embedded systems. However, there is room for improvement on speed, accuracy and memory allocation by customization for a specific problem at the code level. Besides these, CVXGEN is a platform developed to generate custom solvers for small to medium sized quadratic problems (with up to 100s of optimization variables) [57]. However, we cannot directly use the platform as our problem sizes are larger than CVXGEN is designed to handle. Moreover, it is not straightforward to define problems of any sparsity pattern using the platform (especially for quadratic programming (QP) problems with hundreds of optimization variables). The platform is targeted at solving

problems of small to relatively medium size (problems with altogether 2000 coefficients in the constraints and objective) within milliseconds, and cannot guarantee a successful 
code generation once the number of non-zeros in a Karush-Kuhn-Tucker (KKT) matrix goes beyond 4000 [58]. Our problems of trajectory generation are usually large with the total number of non-zeros greater than 4000 in the Karush-Kuhn-Tucker (KKT) matrices. In one of the example in this thesis from Chapter 4 in Section 4.4.1 the number of non-zeros in the Karush-Kuhn-Tucker (KKT) matrices is a little over 7000, and the number of non-zeros in the Karush-Kuhn-Tucker (KKT) matrices for the an example in Section 4.4.3 is a little over 11000. However, CVXGEN is one of the examples that shows the possibility of a significant reduction in solution time of quadratic programming (QP) problems by customization of quadratic programming (QP) solver at the code level. The reference ([57] Section 5.2) suggests that the primal-barrier method in a primal-dual algorithm with Mehrotra Predictor-Corrector tends to have consistent performance over a range of problems. Additionally, Boyd et al. suggest that a convex problem with an interior point method can converge reliably within 25 iterations with reasonable accuracy [62]. Therefore, the algorithm by Vandenberghe [65] was chosen based on a primal-dual interior-point method with Mehrotra Predictor-Corrector to solve general quadratic programming (QP) problems, using a numerical solution strategy similar to [57] (static and dynamic regularizations). Then, a program is developed in the Matlab environment to generate a custom quadratic programming (QP) solver (usable for an embedded application) for a quadratic programming $(\mathrm{QP})$ problem from small to relatively large size (so far the largest quadratic programming (QP) problem solved using the custom code generated from the program has 1028 optimization variables with 36570 non-zeros in the Karush-Kuhn-Tucker (KKT) matrices). Our code generation program takes in the Hessian, Gradient and the Jacobians of a quadratic programming (QP) problem directly, thus it takes record of the sparsity patterns of the problem and generates a custom code accordingly. While the aim of CVXGEN seems to be able to solve small and relatively medium sized quadratic programming (QP) problems within milliseconds, the focus of our customization is to lower the computation time for large problems (namely trajectory generation with obstacle avoidance) using a similar algorithm. The solver thus generated is free from external libraries which is ready for an embedded platform. The reason behind using a library-free code is to avoid constant calling of library functions such as the function to perform permutation, symbolic factorization, matrix conversions, and other supporting sub-functions, which consumes significant computation time. Moreover, the custom solver written in $\mathrm{C}$ uses a set of customized optimization parameters (which can 
be defined before or after the code generation) and has expressions to solve a quadratic programming (QP) with only four mathematical operators $\left(+,-,{ }^{*}, /\right)$.

The quadratic approximation (2.10) may resemble a quadratic programming (QP) problem of the following form [65] where $\mathrm{Q}$ is positive semi-definite (assuming you have a nice feasible point, based on experience).

$$
\begin{array}{cc}
\text { Min } & \frac{1}{2} Z^{T} Q Z+f^{T} Z \\
\text { S.t } & E Z=b \\
& L Z \leq h \\
& \underline{Z} \leq Z \leq \bar{Z}
\end{array}
$$

Although there are various quadratic programming (QP) algorithms, those that can be easily customized for an onboard application for a UAV with minimal programming code are of interest. Such an algorithm would have an advantage of implementation in computational platforms which require no human intervention (embedded application) with less computational capability. One such algorithm is the Primal Barrier Method in the Primal-Dual algorithm with Mehrotra Predictor-Corrector by Vandenberghe [65]. It has been shown in CVXGEN [57], that with enough customization the algorithm is suitable to converge rapidly for small problems. However, our goal is to develop a custom solver generation program that generates custom solver to solve the quadratic programming (QP) problem in order to reduce computation time for our problem at hand (which are usually large quadratic programming (QP) problems). The following section presents a summary of the customization adapted to make a custom solver generation program.

For the problem (2.12), let $Z \in \mathbb{R}^{m}$, and let us introduce slack variable $s \in \mathbb{R}^{j}$ to the inequality constraint and rearrange the problem as,

$$
\begin{array}{cc}
\text { Minimize } & \frac{1}{2} Z^{T} Q Z+f^{T} Z \\
\text { S.t } & E Z=b \\
& \bar{L} Z+s=\bar{h} \\
& s \geq 0
\end{array}
$$


where $\bar{L}=\left[\begin{array}{c}L \\ I \\ -I\end{array}\right], \bar{h}=\left[\begin{array}{c}h \\ \bar{Z} \\ \underline{\underline{Z}}\end{array}\right]$.

Assigning dual variables $p \in \mathbb{R}^{k}$ and $q \in \mathbb{R}^{j}$ associated with the equality and inequality constraints respectively, the Karush-Kuhn-Tucker (KKT) system of equations for problem (2.13) are,

$$
\begin{array}{cc}
\bar{L} Z+s=\bar{h}, & s \geq 0 \\
E Z=b, & \\
Q Z+f^{T}+\bar{L}^{T} q+E^{T} p=0, & q \geq 0 \\
q_{i} s_{i}=0, & i=1, . ., j
\end{array}
$$

From [65], the quadratic programming (QP) algorithm requires two solves with a Karush-Kuhn-Tucker (KKT) system (the linearized KKT system (2.18)) per quadratic programming (QP) iteration besides the initialization at the very beginning of the algorithm that requires an approximate solution to the Karush-Kuhn-Tucker (KKT) system. Thus, the majority of the computational effort in solving problem (2.13) goes towards finding the solution of the Karush-Kuhn-Tucker (KKT) system (2.18). Hence, only the steps taken to design the custom solver to solve the Karush-Kuhn-Tucker (KKT) system are described. First, the initialization is discussed which provides a good starting point to solve the Karush-Kuhn-Tucker (KKT) equations (2.14). The initialization is followed from ([65] Section 5.3) as follows. Although $\hat{Z}$ may come from a preprocessed data supplied by an user, it may not satisfy the equality constraint $E \hat{Z}=b$. Also, $\hat{s}$ obtained from $\bar{L} \hat{Z}+\hat{s}=\bar{h}$ (one of the KKT conditions) may result in the slack variable $\hat{s}$ being negative in case of an infeasible obstacle constraint. Moreover, the dual starting points $[\hat{q}, \hat{p}]$ are unknown. Therefore, in order to have a feasible starting point for the Karush-Kuhn-Tucker (KKT) equations it is suggested to solve (2.15) such that $\hat{Z}, \hat{p}$ and $\hat{q}$ are obtained.

$$
\left[\begin{array}{ccc}
Q & E^{T} & \bar{L}^{T} \\
E & 0 & 0 \\
\bar{L} & 0 & -I
\end{array}\right]\left[\begin{array}{c}
\hat{Z} \\
\hat{p} \\
\hat{q}
\end{array}\right]=\left[\begin{array}{c}
-f^{T} \\
b \\
\bar{h}
\end{array}\right]
$$

Thus, $\hat{Z}, \hat{p}$ and $\hat{q}$ become initial estimates for the Karush-Kuhn-Tucker (KKT) system of 
equations. However, the initialization is not complete yet. In (2.15) $\bar{L} \hat{Z}-\hat{q}=\bar{h}$ is solved instead of $\bar{L} \hat{Z}+\hat{s}=\bar{h}$. This does not affect the nature of solution as it is done only for initialization. A method for solving (2.15) can be obtained from ([65] Section 10). After $[\hat{Z}, \hat{q}, \hat{p}]$ are obtained, $\hat{s}$ and $\hat{q}$ are initialized as,

$$
\hat{s}= \begin{cases}(\bar{h}-\bar{L} \hat{Z}), & \text { if } \alpha_{p}<0 \\ (\bar{h}-\bar{L} \hat{Z})+\left(1+\alpha_{p} \boldsymbol{e}\right), & \text { otherwise }\end{cases}
$$

where, $\alpha_{p}=\inf \{\alpha \mid(\bar{h}-\bar{L} \hat{Z})+\alpha \boldsymbol{e} \geq 0\}, \boldsymbol{e}$ is a column vector that contains all ones with length $j$, and

$$
\hat{q}= \begin{cases}(\bar{L} \hat{Z}-\bar{h}), & \text { if } \alpha_{d}<0 \\ \bar{L} \hat{Z}-\bar{h})+\left(1+\alpha_{d} \boldsymbol{e}\right), & \text { otherwise }\end{cases}
$$

where, $\alpha_{d}=\inf \{\alpha \mid(\bar{L} \hat{Z}-\bar{h})+\alpha \boldsymbol{e} \geq 0\}$.

From the initialization it can be seen that $\hat{q}$ and $\hat{s}$ are both chosen such that $\hat{q}>0$ and $\hat{s}>0$, which means $\hat{q}$ and $\hat{s}$ satisfy the Karush-Kuhn-Tucker (KKT) conditions. Also, there is an advantage in doing so. Let us consider a case when a point in $\hat{Z}$ leads to one of the inequality constraint in (2.12) to become $\bar{L}_{i} Z \geq h_{i}$. If the slack variable is computed in such a case, then it becomes $s_{i}<0$. Therefore, by choosing the initialization shown above, slack variable is set $s_{i}>0$ and then solve for $\hat{Z}$ together with slack and dual variables using Karush-Kuhn-Tucker (KKT) conditions (2.14), so that the new point in $\hat{Z}$ at least leads to the boundary of the inequality, and it does satisfy the inequality as $\bar{L}_{i} Z \leq h_{i}$. Thus, if points in $\hat{Z}$ happen to be defined such that they cause inequality to becomes infeasible, then by the initialization adopted, the primal and dual are set such that the algorithm finds a feasible solution. In addition, the initial estimates $(\hat{Z}, \hat{s}, \hat{p}, \hat{q})$ are improved during quadratic programming (QP) iterations.

After the initialization of the primal and dual variables, the algorithm precisely as shown in Section 5.1 of [65], with Mehrotra correction is followed. Since the aim to develop a custom solver generation program is to accommodate general quadratic programming (QP) problems of relatively larger size (compared to CVXGEN) in order to lower the solution time, the fine details of the algorithm are not discussed, instead the steps adopted for solving the Karush-Kuhn-Tucker (KKT) system of equations in the custom solver are 
presented. A similar treatment for customization of the Karush-Kuhn-Tucker (KKT) solver can be found in $[57,59]$.

In order to make the algorithm applicable for an embedded application, it has to be customized such that the solver does not run into memory overflow and computational instability issues, such as division by zero. These problems especially arise during matrix factorization $\left(L D L^{T}\right)$ and the solving of the Karush-Kuhn-Tucker (KKT) equations. Therefore, during the customization, operations that require division during factorization are protected from going towards zero, at the same time, all the variables that go into the algorithm are predefined (size and data type) and mathematical expressions are hard-coded, thus protecting memory overflow. Once an initial estimate are available as $[\hat{Z}, \hat{s}, \hat{q}, \hat{p}]$, the iteration in the algorithm starts in which the Karush-Kuhn-Tucker (KKT) equations (2.14) are linearized at the initial estimate and solved for an update. Upon linearization around the initial estimates, the Karush-Kuhn-Tucker (KKT) system of equations takes the following form,

$$
\left[\begin{array}{cccc}
Q & 0 & \bar{L}^{T} & E^{T} \\
0 & \operatorname{diag}(\hat{q}) & \operatorname{diag}(\hat{s}) & 0 \\
\bar{L} & I & 0 & 0 \\
E & 0 & 0 & 0
\end{array}\right]\left[\begin{array}{c}
\Delta Z \\
\Delta s \\
\Delta q \\
\Delta p
\end{array}\right]=\left[\begin{array}{c}
-Q \hat{Z}-\bar{L}^{T} \hat{q}-E^{T} \hat{p}-f^{T} \\
-\hat{q} \hat{s} \\
-\bar{L} \hat{Z}-\hat{s}+\bar{h} \\
-E \hat{Z}+b
\end{array}\right]
$$

where $\Delta Z=Z-\hat{Z}, \Delta s=s-\hat{s}, \Delta q=q-\hat{q}$, and $\Delta p=p-\hat{p}$.

Suppose $K l=r$ represents equation (2.18), where $K$ is the Karush-Kuhn-Tucker (KKT) matrix, $l$ and $r$ represent the left hand side vector and the right hand side of the linearized Karush-Kuhn-Tucker (KKT) equations respectively. The Karush-KuhnTucker (KKT) matrix represented by $K$ is symmetrized by multiplying the second row of equation (2.18) with $(\operatorname{diag}(\hat{s}))^{-1}(\hat{s}$ is initialized as greater than zero), which makes the $K$ block $2 \times 2$ symmetric matrix as [57],

$$
\left[\begin{array}{cc|cc}
Q & 0 & \bar{L}^{T} & E^{T} \\
0 & \operatorname{diag}\left(\hat{s}^{-1} \hat{q}\right) & I & 0 \\
\hline \bar{L} & I & 0 & 0 \\
E & 0 & 0 & 0
\end{array}\right]\left[\begin{array}{c}
\Delta Z \\
\Delta s \\
\Delta q \\
\Delta p
\end{array}\right]=\left[\begin{array}{c}
r_{x} \\
r_{s} \\
r_{q} \\
r_{p}
\end{array}\right]
$$


where $r_{x}, r_{s}, r_{q}, r_{p}$, represent the respective right-hand side components of the KarushKuhn-Tucker (KKT) system of equations from (2.18) after the $K$ matrix is symmetrized. The symmetric $K$ matrix is quasisemidefinite (with $(1,1)$ block diagonal being the positive semidefinite and $(2,2)$ block diagonal being the negative semi-definite) in nature therefore the $K$ may not always be factorable. Hence, the $K$ matrix is regularized such that a small number $\epsilon_{k}>0$ is added to the diagonal entries of the $(1,1)$ block, and similarly the same number $\epsilon_{k}>0$ is subtracted from the diagonal entries of the $(2,2)$ block thus making the regularized $K$ matrix as quasidefinite (with $(1,1)$ block diagonal being the positive definite and $(2,2)$ block diagonal being the negative definite), for which the $L D L^{T}$ factorization exists for any permutation matrix [66]. This process of regularization is called static regularization in [57], and can be expressed as follows,

$$
\hat{D}_{i i}= \begin{cases}D_{i i}+\epsilon_{k} & \text { if } i i \in(1,1) \text { block diagonal } \\ D_{i i}-\epsilon_{k} & \text { if } i i \in(2,2) \text { block diagonal }\end{cases}
$$

where $D_{i i}$ and $\hat{D}_{i i}$ represent the current $i^{\text {th }}$ diagonal entry and the updated $i^{\text {th }}$ diagonal entry of the Karush-Kuhn-Tucker (KKT) matrix respectively. The choice of $\epsilon_{k}$ is generic. However, it has been shown that the value of $\epsilon_{k}$ plays a vital role in convergence [57].

Let the regularized $K$ matrix be denoted by $\tilde{K}$. A suitable permutation of $\tilde{K}$ is computed using the Approximate Minimum Degree (AMD) heuristic [67]. The Approximate Minimum Degree (AMD) heuristic algorithm computes a permutation matrix such that the permutation matrix minimizes the additional non-zero entries in the lower-triangular matrix $L$ during permuted- $L D L^{T}$ factorization, thus lowering the floating point operations required. The permuted- $L D L^{T}$ factorization of $\tilde{K}$ can be represented as follows,

$$
\tilde{K}=P L D L^{T} P^{T}
$$

where $P$ is a suitable permutation matrix, $L$ is a lower-triangular matrix, $D$ is a diagonal matrix, and $L$ and $D$ are the $L D L^{T}$ factors of $\tilde{K}$.

The permuted- $L D L^{T}$ factorization of the $\tilde{K}$ may be prone to division by zero operations during factorization when diagonal entries of the $D$ matrix approach near zero which may occur due to matrix scaling issues. Therefore, to avoid the diagonal entries approaching zero during factorization, the following regularization is adopted, also known as dynamic regularization in $[57,59]$. The dynamic regularization does a similar task 
as compared to static regularization. However, it is performed during the factorization process as follows,

$$
\hat{D}_{i i}=S_{i} \delta \text { if } S_{i} D_{i i} \leq \delta
$$

where $D_{i i}$ and $\hat{D}_{i i}$ represent the current $i^{\text {th }}$ diagonal entry and the updated $i^{\text {th }}$ diagonal entry of $D$ matrix during factorization, $S_{i}$ is the sign of $i^{\text {th }}$ diagonal entry $\left(D_{i i}\right)$, and $\delta>0$.

Given the original Karush-Kuhn-Tucker (KKT) system of equations written as $K l=$ $r$, after regularizations $\tilde{K} l=r$ is solved. Now to recover the solution to the original system $K l=r$, where the factorization of $K$ is not available, $\tilde{K} l=r$ is written as $(K+\triangle K) l=r$. Then, the error equation $\triangle K l=(r-K l)$ is obtained. Since the inverse of $\tilde{K}$ is available from the $L D L^{T}$ factorization, $\tilde{K} \delta l=\triangle K l=(r-K l)$ is used instead to solve for $\delta l$, where $l$ is updated iteratively. More details on the iterative refinement process can be found in Duff et al ([68], Section 12.8).

The iterative refinement process is summarized in the following algorithm.

1. solve: $\tilde{K} l=r$ (solution obtained from above)

2. solve for $\delta l: \delta l=\tilde{K}^{-1}(r-K l)$

3. Update: $l=l+\delta l$

4. Repeat steps 2 and 3 until $\|K l-r\| \leq \tilde{\epsilon}$ becomes sufficiently small for $\tilde{\epsilon}>0$.

The refinement precision can be set during the code generation process, and the convergence of the iterative refinement with this particular $\tilde{K}$ to $K$ can be found in reference ([68], Section 12.8). (At each quadratic programming (QP) iteration KarushKuhn-Tucker (KKT) equations are re-linearized.)

For a custom solver generation, the structure of $K$ in (2.18) should be known in advance. In fact, as the components of $K$ in (2.18) come from the Jacobians, Hessian and the gradient of problem (2.12), which are calculated by the first and the second order Taylor approximation of the constraints and cost function respectively, the locations of the non-zero terms in $K$ are known in advance and are fixed for a given problem. Also, the location of non-zeros due to static regularization of the Karush-Kuhn-Tucker (KKT) system from (2.20) are known as well. Thus, with the fixed known structure of $\tilde{K}$ (after static regularization on $K$ ) a permutation $P$ (to $\tilde{K}$ ) is computed using Approximate Minimum Degree (AMD) heuristics. The relative magnitudes of the non-zero terms on $\tilde{K}$ 


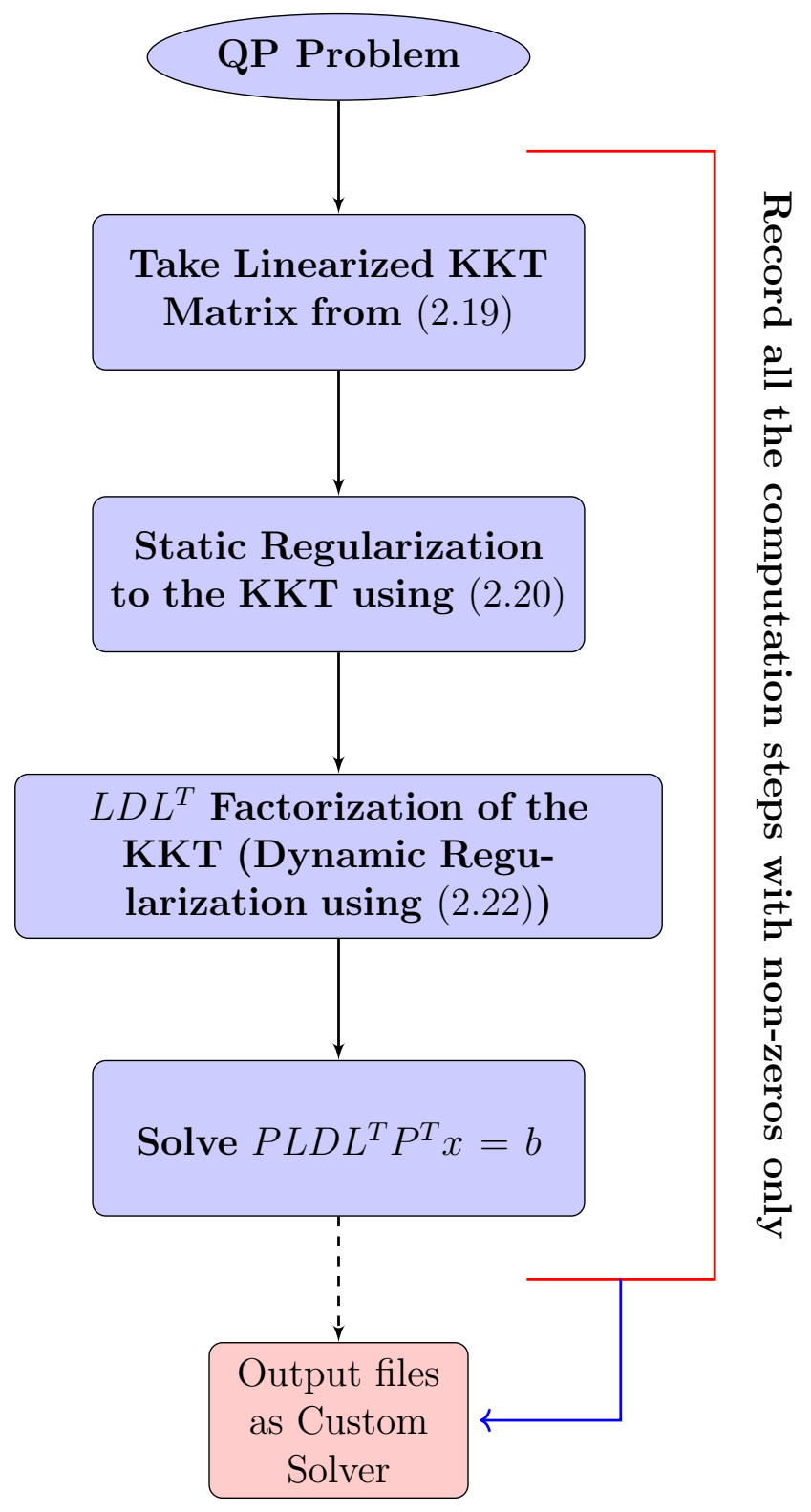

Figure 2.2: Custom solver generation

may not be the same, but it does not affect the outcome (factors $D$ and $L$ ) resulting from the use of a permutation matrix. Moreover, the use of the permutation matrix maintains the structure of the $L D L^{T}$ factorization. Now, with a fixed and known $P$ and $\tilde{K}$, expressions for $L$ and $D$ are found using $L D L^{T}$ factorization (in a reverse manner). Also, to prevent the division by zero during the factorization the dynamic regularization (2.22) 
for each component of the factor $D$ (of $\tilde{K}$ ) is hard-coded. The flowchart in Figure 2.2 shows the steps summarized in this paragraph for custom Karush-Kuhn-Tucker (KKT) solver generation. Since, the location of non-zeros in $(\tilde{K}, L$ and $D)$ are recorded at each step during the code generation, and the expressions are identified that have operations with non-zeros, the custom Karush-Kuhn-Tucker (KKT) solver for a quadratic programming (QP) problem with any sparsity pattern can be generated. A resulting compiled custom solver has fixed known expressions to find factors $L$ and $D$, fixed expressions for matrix-vector and matrix-matrix operations, and fixed expressions for the solve process. Therefore, with fixed-known expressions at every step, the approximate solution time can be fairly reliably known.

Thus using the quadratic cone algorithm from [65], and customization to solve KarushKuhn-Tucker (KKT) equations from [57, 59] (the static and the dynamic regularization

), a custom solver is generated, with fixed known memory, hardcoded in C programming language and free from programming overhead or library functions, to solve the problem (2.13) until the Karush-Kuhn-Tucker (KKT) system of equations (2.14) are solved to the desired accuracy.

\subsection{Example of Customization}

In the following an example of the method of customization is shown. Let us consider a matrix $\mathrm{A}$ as the following,

$$
A=\left[\begin{array}{llll}
a & b & 0 & 0 \\
b & c & d & 0 \\
0 & d & 0 & e \\
0 & 0 & e & f
\end{array}\right]
$$

The matrix A is taken as symmetric, and for the purpose of customization let us suppose it to be positive semi-definite. When the Karush-Kuhn-Tucker (KKT) system of equations in (2.18) is observed, the system of equations is of the form $K l=r$. First, the right hand side $r$ contains matrix-vector operations, and the matrices are usually sparse in nature. Therefore, a customization is needed with matrix-vector operation to avoid unnecessary computation with zeros. Second, the system $K l=r$ itself has to be 
solved, for which the permuted $L D L^{T}$ factorization is used. As stated earlier in this chapter two factorizations per quadratic programming (QP) iteration are required which are computationally expensive. Therefore, customization with the solution of the system $K l=r$ is required.

\subsubsection{Matrix-Vector Multiplication}

First, a customization for matrix-vector operation is shown. For that, the non-zeros of $\mathrm{A}$ are written in a row vector but the components are listed column-wise as,

$$
A_{c}=\left[\begin{array}{lllllllll}
a & b & b & c & d & d & e & e & f
\end{array}\right]
$$

Let $\mathrm{x}$ and $\mathrm{y}$ be vectors of length 4 , then a custom matrix-vector operation $y=A_{c} x$ is written as,

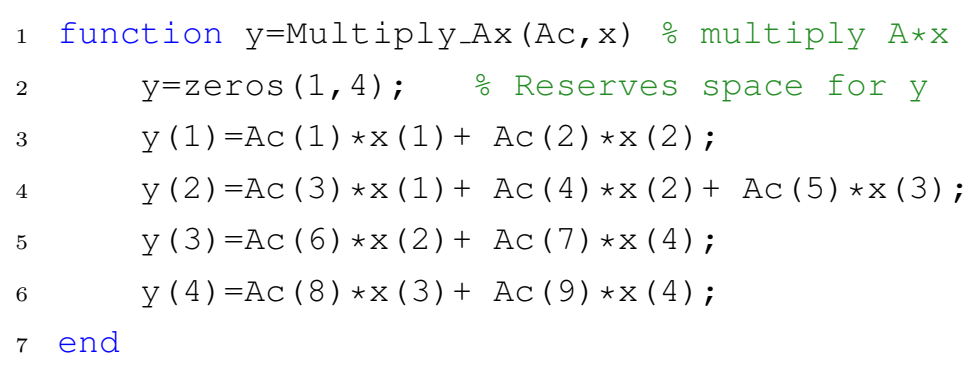

The above matrix-vector operation eliminates the operation with zeros from the matrix A. Also, the memory for storing $A_{c}$ is lower than storing $\mathrm{A}$, and for the case of a symmetric matrix only the lower-triangular non-zeros are needed to be stored. This customization is significant for large sparse matrices, especially in an embedded system where memory and processing capabilities are limited. Thus, for the above example case $A_{s}$ can be stored as,

$$
A_{s}=\left[\begin{array}{llllll}
a & b & c & d & e & f
\end{array}\right]
$$

And, write a custom matrix-vector operation $y=A_{s} x$ as,

1 function $y=\operatorname{Multiply\_ Ax}(\mathrm{As}, \mathrm{x}) \div \operatorname{multiply} \mathrm{A} * \mathrm{x}$ 


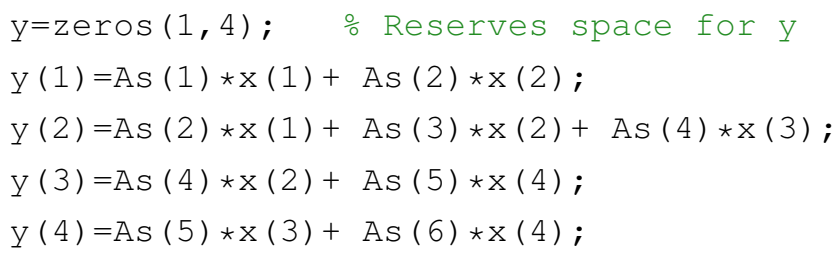

Although the functions in the above examples due to (2.24) and (2.25) may require the similar sized memory storage but it takes less memory to store $A_{s}$ in $(2.25)$ compared to $A_{c}$ in $(2.24)$.

\subsubsection{Custom KKT solver}

In order to build a custom Karush-Kuhn-Tucker (KKT) solver, a custom $L D L^{T}$ factorization of the Karush-Kuhn-Tucker (KKT) matrix, and then a custom solve of the Karush-Kuhn-Tucker (KKT) system equation are required.

Given the matrix A in (2.23), a step called static regularization is performed by adding $\delta>0$ in the diagonal entries of $\mathrm{A}[57,59]$.

$$
\tilde{A}=\left[\begin{array}{cccc}
a+\delta & b & 0 & 0 \\
b & c+\delta & d & 0 \\
0 & d & 0+\delta & e \\
0 & 0 & e & f+\delta
\end{array}\right]
$$

Then, the Approximate Minimum Degree (AMD) heuristics is used to find a permutation $P$ [67]. The Approximate Minimum Degree (AMD) heuristics finds a permutation $\mathrm{P}$ such that it would result in the minimum number of non-zeros in the lower-triangular factor $L$, which will subsequently minimize the memory requirement, and the solution time using custom solver. Thus, using the Approximate Minimum Degree (AMD) heuristics for the above $\tilde{A}, P=[4,3,1,2]$ is found, which results in minimum number of non-zeros components in the factor $L$ (resulting in 3 non-zeros, detailed below). The permuted $\tilde{A}$ 
matrix becomes,

$$
P \tilde{A} P^{\prime}=\left[\begin{array}{cccc}
f+\delta & e & 0 & 0 \\
e & \delta & 0 & d \\
0 & 0 & a+\delta & b \\
0 & d & b & c+\delta
\end{array}\right]
$$

The permuted- $\tilde{A}$ is written in a row vector but the components listed column-wise as,

$$
A_{x n s}=\left[\begin{array}{llllllllll}
f+\delta & e & e & \delta & d & a+\delta & b & d & b & c+\delta
\end{array}\right]
$$

However, $A_{x}$ is stored for custom solver as,

$$
A_{x}=\left[\begin{array}{lllllll}
f+\delta & e & \delta & d & a+\delta & b & c+\delta
\end{array}\right]
$$

Since, the permuted- $L D L^{T}$ factorization is used, we can write

$$
P \tilde{A} P^{T}=L D L^{T}
$$

which is equivalent to equation $(2.21), L$ is a lower triangular matrix with unit diagonal and $D$ is a diagonal matrix, and $L$ and $D$ are the factors of the permuted- $\tilde{A}$.

Let us suppose the factors $L$ and $D$ as,

$$
\begin{gathered}
L=\left[\begin{array}{cccc}
1 & 0 & 0 & 0 \\
L_{(2,1)} & 1 & 0 & 0 \\
L_{(3,1)} & L_{(3,2)} & 1 & 0 \\
L_{(4,1)} & L_{(4,2)} & L_{(4,3)} & 1
\end{array}\right] \\
D=\left[\begin{array}{cccc}
D_{(1,1)} & 0 & 0 & 0 \\
0 & D_{(2,2)} & 0 & 0 \\
0 & 0 & D_{(3,3)} & 0 \\
0 & 0 & 0 & D_{(4,4)}
\end{array}\right]
\end{gathered}
$$

To find expression for all the components of $L$ and $D$, fist the components of $L D L^{T}$ are found and then equated with $P \tilde{A} P^{T}$. To concise, let us represent $L_{(x, y)}$ with $L_{x y}$, and 
$D_{(x, x)}$ as $D_{x}$. Thus, with $L$ and $D$ above, we get

$$
\begin{aligned}
& L D L^{\prime}=\left[\begin{array}{llll}
D_{1} & D_{1} L_{21} & D_{1} L_{31} & \ldots \\
D_{1} L_{21} & D_{1} L_{21}^{2}+D_{2} & D_{2} L_{32}+D_{1} L_{21} L_{31} & \ldots \\
D_{1} L_{31} & D_{2} L_{32}+D_{1} L_{21} L_{31} & D_{1} L_{31}^{2}+D_{2} L_{32}^{2}+D_{3} & \ldots \\
D_{1} L_{41} & D_{2} L_{42}+D_{1} L_{21} L_{41} & D_{3} L_{43}+D_{1} L_{31} L_{41}+D_{2} L_{32} L_{42} & \ldots
\end{array}\right. \\
& \left.\begin{array}{l}
D_{1} L_{41} \\
D_{2} L_{42}+D_{1} L_{21} L_{41} \\
D_{3} L_{43}+D_{1} L_{31} L_{41}+D_{2} L_{32} L_{42} \\
D_{1} L_{41}^{2}+D_{2} L_{42}^{2}+D_{3} L_{43}^{2}+D_{4}
\end{array}\right]
\end{aligned}
$$

Now equating $P \tilde{A} P^{T}=L D L^{T}$, the components of $L$ and $D$ in terms of the components of $P \tilde{A} P^{T}$ are,

$$
\begin{gathered}
D_{1}=f+\delta \\
L_{21}=\frac{e}{D_{1}} \\
L_{31}=0 \\
L_{41}=0 \\
D_{2}=\delta-L_{21}^{2} D_{1} \\
L_{32}=0 \\
L_{42}=\frac{d}{D_{2}} \\
D_{3}=a+\delta
\end{gathered}
$$




$$
\begin{gathered}
L_{43}=\frac{b}{D_{3}} \\
D_{4}=c+\delta-D_{2} L_{42}^{2}-D_{3} L_{43}^{2}
\end{gathered}
$$

Although the expression seems straight-forward and simple, there may be cases when the system may not be solvable if the components of $\mathrm{D}$ matrix are close to zero. When the value of $D_{i}$ are closer to or lower than a machine precision, in that case $D_{i}$ may be referred to as zero. In this case, all the components that require the division by $D_{i}$ become Infinite. For example, if $f$ and $e$ are numbers such that they would result $D_{2}$ to be zero, then, all the components of $L$ that requires division by $D_{2}$ turns to be Infinite, such as $L_{42}$. Therefore, to prevent such cases during factorization (which also helps during forward and backward solve with $L D L^{T}$ system), a step called dynamic regularization $[57,59]$ is applied, which prevents the diagonal entries $D_{i}$ getting close to zero. Therefore, after computing a diagonal element (during factorization) there is a check if $D_{i}$ is less than a certain value. If $D_{i}$ turns out to be smaller than a certain value, a number $\delta$ is added which is greater than zero, thus preventing $D_{i}$ getting close to zero. The following condition is inserted after each computation with $D_{i}$.

$$
\begin{aligned}
& \text { if } D_{i} * D_{i} \leq \delta * \delta \\
& \text { if } D_{i}<0 \\
& D_{i}=-\delta \\
& \text { else } \\
& D_{i}=\delta \\
& \text { end } \\
& \text { end }
\end{aligned}
$$

From the above calculation, the factors $L$ and $D$ with their non-zeros entries can be written as,

$$
L=\left[\begin{array}{cccc}
1 & 0 & 0 & 0 \\
L_{21} & 1 & 0 & 0 \\
0 & 0 & 1 & 0 \\
0 & L_{42} & L_{43} & 1
\end{array}\right]
$$




$$
D=\left[\begin{array}{cccc}
D_{1} & 0 & 0 & 0 \\
0 & D_{2} & 0 & 0 \\
0 & 0 & D_{3} & 0 \\
0 & 0 & 0 & D_{4}
\end{array}\right]
$$

We can observe that there are only three non-zero entries besides the diagonal unit entries in $L$, and four entries in $D$. Thus, only the components for non-zeros from the $L$ and $D$ matrix are stored column-wise in row vectors as follows,

$$
\begin{aligned}
& L_{x}=\left[L_{21}, L_{42}, L_{43}\right] \\
& D_{x}=\left[D_{1}, D_{2}, D_{3}, D_{4}\right]
\end{aligned}
$$

where expressions for the components are given above. Then with $\delta=10^{-7}$, a function is written that computes $L D L^{T}$ factorization of permuted- $\tilde{A}$ as factor, shown in Appendix 1.1.

This function factor computes factors $L$ and $D$ for the permuted- $\tilde{A}$ with the minimum floating point operations required. In the function, $L_{x}$ reserves the space for three variables which align with our expression for $L_{x}$. Similarly, $D_{x}$ reserves space for four variables as it has four components. In the function, we observe $D_{x}(1)=A_{x}(1)$ which is equal to $D_{x}(1)=f+\delta$ as shown in (2.27a). Similarly, it can be observed that our expressions to compute the components of $L$ and $D$ in the function factor matches with expressions in equation (2.27). Likewise, in the function the if-condition from (2.28) is inserted after each $D_{i}$ is computed.

Once the factors $L_{x}$ and $D_{x}$ are obtained, then the system like $A x=b_{b}$ can be solved, where $b_{b}$ is a column vector of similar length to the vector $x$. As $P A P^{T}=L D L^{T}$ can be written as $A=P L D L^{T} P^{T}$, then $A x=b_{b}$ is written as $P L D L^{T} P^{T} x=b_{b}$ and solved in the following manner ([62], Appendix C.3),

1. Permutation Solve: $P v_{1}=b_{b}$

Given $\mathrm{P}, v_{1}$ is found as, 


$$
\begin{aligned}
& v_{1}(1)=b_{b}(4) \\
& v_{1}(2)=b_{b}(3) \\
& v_{1}(3)=b_{b}(1) \\
& v_{1}(4)=b_{b}(2)
\end{aligned}
$$

2. Forward Substitution Solve: $L v_{2}=v_{1}$

Given L from (2.29) and $v_{1}$ from above, $v_{2}$ is solved by forward substitution,

$$
\begin{aligned}
& v_{2}(1)=v_{1}(1) \\
& v_{2}(2)=v_{1}(2)-L_{21} v_{2}(1)=v_{1}(2)-L x(1) v_{2}(1) \\
& v_{2}(3)=v_{1}(3) \\
& v_{2}(4)=v_{1}(4)-L_{42} v_{2}(2)-L_{43} v_{2}(3)=v_{1}(4)-L x(2) v_{2}(2)-L x(3) v_{2}(3)
\end{aligned}
$$

3. Diagonal Solve: $D v_{3}=v_{2}$

Given D from (2.30) and $v_{2}$ from above, $v_{3}$ is found as,

$$
\begin{aligned}
& v_{3}(1)=v_{2}(1) / D_{1}=v_{2}(1) / D x(1) \\
& v_{3}(2)=v_{2}(2) / D_{2}=v_{2}(2) / D x(2) \\
& v_{3}(3)=v_{2}(3) / D_{3}=v_{2}(3) / D x(3) \\
& v_{3}(4)=v_{2}(4) / D_{4}=v_{2}(4) / D x(4)
\end{aligned}
$$

4. Backward Substitution Solve: $L^{T} v_{4}=v_{3}$

Given L from (2.29) and $v_{3}$ from above, $v_{4}$ is found as,

$$
\begin{aligned}
& v_{4}(4)=v_{3}(4) \\
& v_{4}(3)=v_{3}(3)-v_{4}(4) L_{43}=v_{3}(3)-v_{4}(4) L x(3) \\
& v_{4}(2)=v_{3}(2)-v_{4}(4) L_{42}=v_{3}(2)-v_{4}(4) L x(2) \\
& v_{4}(1)=v_{3}(1)-v_{4}(2) L_{21}=v_{3}(1)-v_{4}(2) L x(1)
\end{aligned}
$$

5. Permutation Transpose Solve: $P^{T} x=v_{4}$ 
Finally, given $\mathrm{P}, x$ is found as,

$$
\begin{aligned}
& x(4)=v_{4}(1) \\
& x(3)=v_{4}(2) \\
& x(1)=v_{4}(3) \\
& x(2)=v_{4}(4)
\end{aligned}
$$

Although the steps shown above are the operations required to solve the system $A x=b_{b}$, the expressions can be simplified and written as custom function to perform all those operations, and the function for the example is solve, which is shown in Appendix 1.2. In the function solve, all the intermediate variables $v_{1}, v_{2}, v_{3}$ and $v_{4}$ as seen in the above 5 -solve steps are simplified, and then the concise expressions with only non-zero are written.

Although there are many sub-steps for the solution of the system $A x=b_{b}$, we keep a record of all the non-zeros and the expressions to compute them during the code generation process and finally write a custom function that requires minimal memory and the least floating point operations. However, when it comes to a large quadratic programming (QP) problem, one important thing that was noted during the course of customization is the memory footprint of the custom function (program or code) which increases with the size of quadratic problem. Although, it is inevitable and reasonable to expect an increase in file size with an increasing quadratic programming (QP) size, this also increases the function loading time. Therefore, in order to lower the file size, the second approach of customization is presented for solving large Karush-Kuhn-Tucker (KKT) system of equations.

In the first approach, a direct expression for each component of the factors and the solution steps were written. While in the second approach, instead their locations are stored in column-wise form and then use that to shorten the number of operations required to solve a system equation. In order to do that the locations of all the non-zeros are stored in column form in a row vector (as in Davis et. al [69]). Since the locations of the non-zeros of a Karush-Kuhn-Tucker (KKT) matrix are known, the locations of all relevant non-zeros required for the solution of the Karush-Kuhn-Tucker (KKT) system can be found. Therefore, from the locations of the non-zeros of the Karush-Kuhn-Tucker 
(KKT) matrix the permutation $P$ is found that gives the minimum number of non-zeros in the lower-triangular factor $L$ (for $L D L^{T}$ factorization) using Approximate Minimum Degree (AMD) heuristics [67]. Once the locations of the non-zeros in the lower-triangular factor are known then the algorithm is followed for factorization and solve, from Davis [69]. In order to generate a custom factorization and solve steps for a Karush-KuhnTucker (KKT) system, the locations of the non-zeros of the Karush-Kuhn-Tucker (KKT) matrix and its lower-triangular factor are stored which remains consistent for a given problem formulation. Thus, by storing the locations of the non-zeros, we avoid the repetitive computation of the permutation $P$, and the locations of the non-zeros for the factor $L$. Moreover, we can reserve a static memory for these respective vectors, and then pre-determine the solution steps related to them (as similar in the custom function, factor, in the first approach where non-zeros of $L_{x}$ and $D_{x}$ are only utilized).

In the second case, once the permutation for a Karush-Kuhn-Tucker (KKT) system is found, then the custom function is generated, which stores the locations of the non-zeros for the Karush-Kuhn-Tucker (KKT) system as in (2.24). In this case all the non-zeros of a Karush-Kuhn-Tucker (KKT) system needs to be stored despite the symmetricity which only requires storing a the lower-triangular part [69]. This is because, during the factorization process upper triangular part of permuted Karush-Kuhn-Tucker (KKT) matrix are considered, however the elements at the permuted Karush-Kuhn-Tucker (KKT) matrix may come from the lower triangular part of the Karush-Kuhn-Tucker (KKT) system. Therefore, to ensure the factorization of a correct matrix, all the non-zeros of a Karush-Kuhn-Tucker (KKT) matrix are stored. Likewise, the locations of non-zeros for the lower-triangular factor $L$ are stored. As the diagonal factor $D$ contains non-zeros only in the leading diagonal with all being non-zeros, we do not have to store their locations. An example for solving $A x=b_{b}$ is discussed below with the second approach of customization.

In order to solve $A x=b_{b}$, first the factors $L$ and $D$ of $A$ are computed using the custom factorization function in Appendix 1.3. The custom factorization function is generated using the second approach of customization where vectors that are associated with the location of non-zeros of the Karush-Kuhn-Tucker (KKT) system and the lowertriangular factor $L$ are stored. Unlike the first approach where the lines of code increase with the number of non-zeros (which causes an increase in the memory footprint of the function file), in the second approach only the size of row vectors increase (such as Axp, 
Axi, Lxp, Root, Pinv, and P including intermediate vectors and factors shown in the function factor 2 in Appendix 1.3) and this does not drastically increase the memory footprint of the function file for a large quadratic programming problem.

After the factorization comes the solve step, where a custom solve function solve2, shown in Appendix 1.4 is used. As similar to the factorization function, the solve function (solve2) keeps a record of the non-zeros associated with the lower-triangular factor $L$ and the permutation $P$. Therefore, besides the size of output vector, intermediate variable, permutation matrix, and the locations of non-zeros of the $L$ and $A$, the code length remains the same from step 1 to 5 of solve for any size of quadratic programming (QP). Figure 2.3 shows a flowchart for solving a quadratic programming problem using the quadratic programming algorithm from [65] on left hand side. In the algorithm, it requires four solves with the Karush-Kuhn-Tucker (KKT) system at every iteration. If the algorithm is to be used without customization then a significant computational effort would go on computing permutation, factorization and solve steps for the full KarushKuhn-Tucker (KKT) system of equations. From the experience of solving numerical examples considered in this dissertation, a quadratic problem (considered in this dissertation) took more than ten iterations of the quadratic programming algorithm, which means using a customized solver we eliminate the computation of the permutation at least ten times. Moreover, fixed expressions for factorization and solve steps are used, thus significantly reducing solution time.

In the following a comparison between the above two approaches (for custom KarushKuhn-Tucker (KKT) solver generation) are made, in file size which arise in the trajectory generation problem for quad-rotor UAVs as similar to the one that will be solved in Chapter 5.

Table 2.1: File sizes for the two approaches

\begin{tabular}{ccccc}
\hline \hline Problems & KKT Size & Non-zeros & Approach 1 (KB) & Approach 2 (KB) \\
\hline 1 & 536 & 1936 & 287 & 82 \\
2 & 1541 & 5611 & 954 & 251 \\
3 & 2613 & 9531 & 1676 & 435 \\
4 & 9983 & 36481 & 6127 & 1651 \\
5 & 16683 & 60981 & 10620 & 2866 \\
\hline \hline
\end{tabular}

Table 2.1 shows the comparison in the file size of the custom Karush-Kuhn-Tucker 


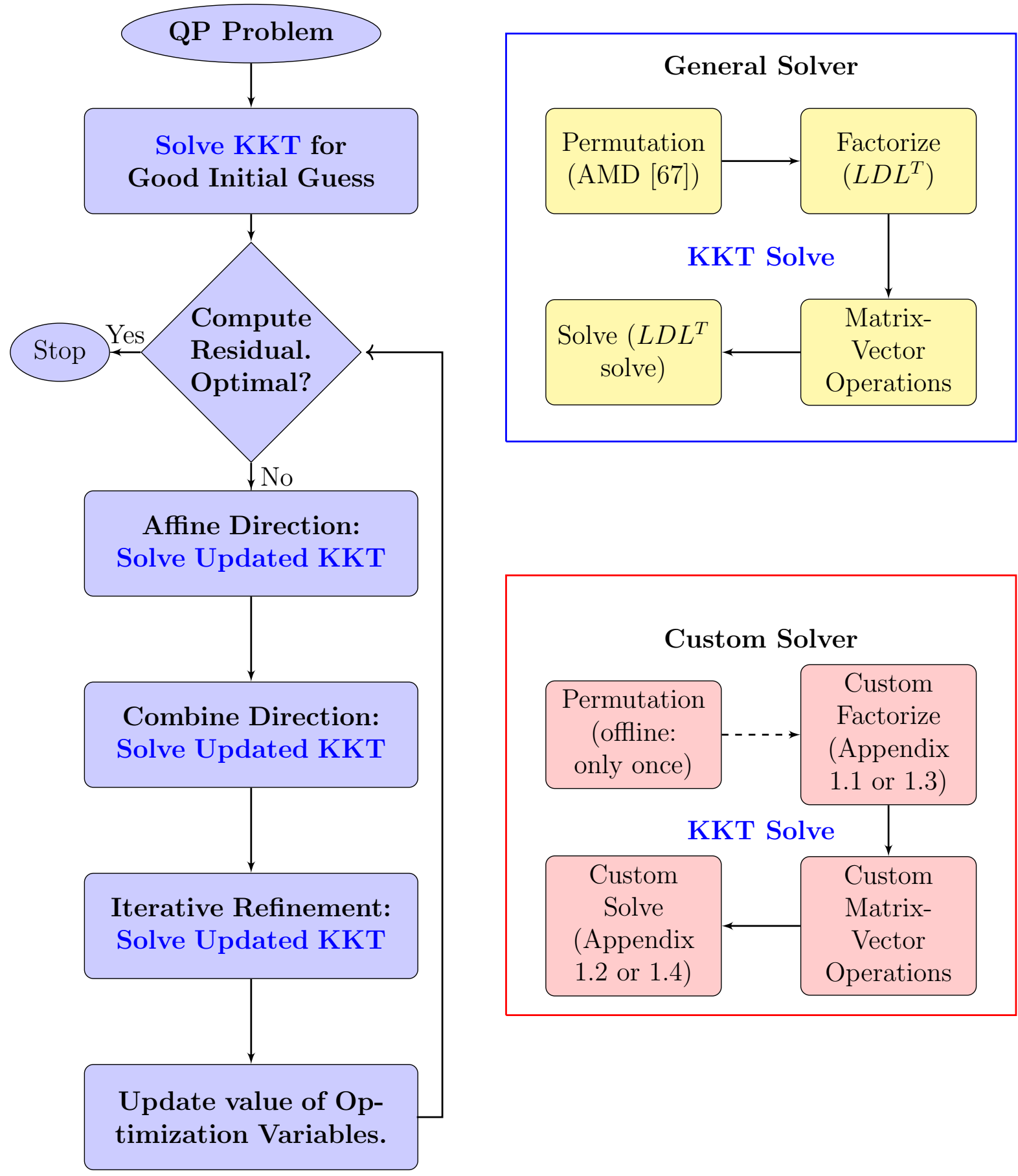

Figure 2.3: General solver and custom solver comparison 
(KKT) solver between the first and the second approach of customization. In the Table 2.1, the file size of the custom solver function using the first approach is at least more than three times the file size with the second approach. Although the file size of the custom solver function depends on the size of a Karush-Kuhn-Tucker (KKT) matrix and the non-zeros associated with it, in our experience the location of non-zeros in the Karush-Kuhn-Tucker (KKT) matrix away from diagonal entries affects the file size the most (increases the file size). In a comparison between two Karush-Kuhn-Tucker (KKT) systems with the same size, it was found that the function file size of a custom solver for the Karush-Kuhn-Tucker (KKT) with non-zeros away from diagonal entires is higher compared to the one with non-zeros entries close to the diagonal.

Therefore, a Karush-Kuhn-Tucker (KKT) system with most of the non-diagonal elements close to the leading diagonal gets benefited from the first approach of customization. While the second approach is a general approach for custom code generation for large Karush-Kuhn-Tucker (KKT) system with any sparsity pattern.

\subsection{Summary}

This chapter presented two approaches of customization of a Karush-Kuhn-Tucker (KKT) solver for a quadratic programming (QP) problem with the positive semi-definite Hessian. The main computational effort during the custom solver generation goes in storing intermediate expressions which have to be recorded and finally written in a file as a function. In our experience, a large Karush-Kuhn-Tucker (KKT) system with non-zeros away from the diagonal entries takes much computational time and large function file size using the first approach of customization compared to with the second approach. Although the second approach of customization offers less memory requirement for a large Karush-Kuhn-Tucker (KKT) system, the first approach instead stores the direct expressions for computing the factors and the solve steps to a Karush-Kuhn-Tucker (KKT) system. Therefore, the first approach is beneficial for small sized quadratic programming (QP) problems. However, due to the size of the problems considered in this thesis, which are large, the second approach of customization is used. 


\section{Chapter 3}

\section{Rapid Feasible Trajectory Generation for Collision Avoidance in Fixed-wing UAVs}

In Chapter 2, the methods of customization and approaches for custom solver generation are shown. The custom solver thus generated eliminates the need for dynamic memory and the use of library functions thus speeding the solution process. Thus, an aim is to use a custom solver generated from the approach detailed in Chapter 2. Next, for the case of fixed-wing unmanned aerial vehicles (UAVs), which have a minimum speed greater than zero, rapid trajectory generation and updating of environment (obstacles and terrain) information are required to fly safely. Therefore, in this chapter, a method for feasible trajectory generation for fast-paced environments is proposed. The purpose of rapid feasible trajectory generation disregarding optimality is to ensure a feasible trajectory, so that a UAV may fly safely among obstacles even though an optimal solution, which is generally computation-intensive, may not be available. In the proposed approach, dynamic aircraft constraints are first simplified and then defined as functions of points in three-dimensional space and velocity, using which a nonlinear programming problem is defined to obtain a feasible set of state-control pairs. The sequential convex programming (SCP) method utilizing the custom solver presented in Chapter 2 is then used to solve this nonlinear programming problem. Numerical results from the approach show that the use of the custom solver reduces the computation time to tens of milliseconds, 
making the approach applicable for rapid feasible trajectory generation. Moreover, a feasible trajectory can also be used as an initial guess for an optimal trajectory generation problem, to obtain an optimal solution with significantly reduced computation time, compared to solving it directly.

\subsection{Introduction}

Onboard trajectory generation for autonomous obstacle avoidance is one of the sought after capabilities for fixed-wing UAVs. However, the computational demand for obstacle free trajectory generation varies with methods, and the computationally demanding methods are less likely to be placed onboard micro UAVs. There are several studies concerned with trajectory generation for UAVs, and survey are presented in [70, 71, 72]. Most trajectory generation methods for the constrained UAV problem adopt either an optimization approach or a certain type of iterative approach $[72,73,41,74]$. In particular, the cell decomposition method [75], the road map method [76], the artificial potential field method [77, 78, 13, 14], the rapidly exploring random tree method [79, 80, 39, 81], and transcription methods $[19,27,28,21,34,82]$. Other methods include linear interpolation [83] and B-spline parameterization [84]. Most of these studies do not address the computation time issue, and some of the methods are overly computationally intensive for real-time application. As far as this thesis is concerned, a feasible path generation method for fixed-wing aircraft was proposed by Bakolas et al. [85], where a geometric approach is used for defining a flight path that is then time parameterized to form a trajectory by solving an nonlinear programming (NLP) problem. As that study was not aimed at online trajectory generation, rapid solvability of the problem was not a concern. Regarding the solution time for feasible trajectory generation, a custom solver to reduce computation time appeared in [86], where CVXGEN [58] was used to generate the solver. However, the results in the reference for real-time usability cannot be compared as the approach is for trajectory planning for swarm application.

As this chapter presents an approach for rapid feasible trajectory generation for fixedwing UAVs, the problem of trajectory generation for a fixed-wing UAV is defined as, given an initial point the UAV has to fly through an obstacle-rich environment where it has the goal point to reach by avoiding obstacles, satisfying state and control constraints, 
and fulfilling mission objectives. The problem is posed as an optimal control problem which is then simplified so that the simple problem can be solved more rapidly than the original problem. The simplified version of the trajectory generation problem is defined as functions of the $x, y, z$ points in $3 \mathrm{D}$ space, along with velocity, $v$. The definition of a feasible trajectory generation problem with four optimization parameters per point in space leads to a low solution time compared to the original problem with more than four optimization parameters for each point in space. A somewhat similar idea also appears in [85], where a semi-analytical method for initial guess generation was proposed. It was then used in time optimal trajectories generation. In that study, the process of initial guess generation was divided into two steps. In the first step, path primitives (Dubins patterns) were used to generate a path by solving the path planning problem in vertical and horizontal planes separately, resulting in a geometric path. In the second step, the path is then time parametrized using inverse dynamics, where flight parameters such as velocity and flight angles are functions of the position in $3 \mathrm{D}$ space. The goal of the second step in the paper was to find a velocity profile such that the aircraft could follow the path without violating control constraints. By contrast, in this chapter, states, controls, and constraints are parameterized as functions of position and velocity in space and are solved as a single problem to generate time parameterized feasible trajectories in the presence of obstacles with some objectives. In the proposed approach for obstacle avoidance, a geometric relation (a relation based on the position of points in space) is considered between the UAV and an obstacle location. Reference [85] solves the problem of an initial guess generation to be used for landing trajectory generation for an aircraft in two stages where the problem does not have obstacles to consider, and the reference does not discuss the real-time usability of the approach. Whereas, we deal with a problem of a feasible trajectory generation for a UAV from an initial point to a goal point, avoiding stationary and moving obstacles, while remaining close to a reference path and satisfying control saturation. Thus, the present differs in terms of problem definition and our expected application (real-time usability). Also, in the present study the problem of a feasible trajectory generation is solved in a single stage, with its capability of real-time usability.

The remainder of this chapter is organized as follows. In Section 3.2, the problem is stated and the simplification of a dynamic equation for a fixed-wing UAV is presented. Section 3.3 presents the method for feasible trajectory generation, and in Section 3.4, a Monte-Carlo test and a numerical example are presented. Finally, Section 3.5 summarizes 
the chapter.

\subsection{Problem Formulation}

In this section, the problem of trajectory generation and autonomous collision avoidance for a fixed-wing UAV is stated. Let $X=(x, y, z, v, \gamma, \psi)$ represent the state variables, where $(x, y, z)$ refers to position in space, $v$ denotes the ground speed (with the assumption of still air), $\psi$ denotes the yaw angle, and $\gamma$ denotes the flight path angle. Let $(\alpha, \phi, T)$ be the control parameters, where $\alpha$ is the angle of attack, $\phi$ is the bank angle, and $T$ is the thrust of the aircraft. For the considered trajectory generation, only the motion of the vehicle is of interest. Therefore, as suggested by Feldman ([87], Chapter 2 ), the dynamic model with assumptions that a fixed-wing UAV is a point mass model, in symmetric flight, with no any lateral force and no sideslip, and without regard to wind field from $[88,89,90]$ is adopted as follows,

$$
\left[\begin{array}{c}
\dot{x} \\
\dot{y} \\
\dot{z} \\
\dot{v} \\
\dot{\gamma} \\
\dot{\psi}
\end{array}\right]=\left[\begin{array}{c}
v \cos (\psi) \cos (\gamma) \\
v \sin (\psi) \cos (\gamma) \\
v \sin (\gamma) \\
\frac{1}{M}[-D+T \cos (\alpha)-M g \sin (\gamma)] \\
\frac{1}{M v}[L \cos (\phi)+T \sin (\alpha) \cos (\phi)-M g \cos (\gamma)] \\
\frac{1}{M v \cos (\gamma)}[L \sin (\phi)+T \sin (\alpha) \sin (\phi)]
\end{array}\right]
$$

where $M$ is the mass of the aircraft, $L$ (lift force) $=\frac{1}{2} \rho v^{2} S C_{L}, D$ (Drag force) $=$ $\frac{1}{2} \rho v^{2} S C_{D}, g$ is gravitational acceleration $\left(9.81 \mathrm{~m} / \mathrm{s}^{2}\right), C_{D}=C_{D_{0}}+K C_{L}^{2}$ is the drag coefficient, $C_{L}=C_{L_{\alpha}} \alpha$ is the lift coefficient, and $K=\frac{1}{\pi A e}$. The wing surface area is $S, A$ is the aircraft's aspect ratio given by $A=\frac{b^{2}}{S}$, where $b$ is the wing span, $e$ is the Oswald efficiency, and $\rho$ is air density. In Equation (3.1), the effect of thrust $(T)$ has been added to the model derived in ([89], Chapter 3). In (3.1) the thrust line is assumed to be in line with the zero-lift line $(\alpha=0)$ of the aircraft. However, this is not the case for the aircraft that use thrust-augmented lift.

Given the vehicle dynamics above, the problem can now be stated. It is assumed that a fixed-wing UAV starts at time $t_{0}$ (initial time) from the initial position $\left(x_{0}, y_{0}, z_{0}\right)$ with 
velocity, flight path angle and heading angle as $v_{0}, \gamma_{0}$ and $\psi_{0}$ respectively, and at the final time $t_{f}$ (free final time) it should reach the target point $\left(x_{f}, y_{f}, z_{f}\right)$ while flying low among obstacles. Moreover, there is a desired path that the UAV should closely follow throughout the flight. This path is represented by a straight line at a certain height $z_{d}$. Its choice is generic, as it may represent a line connecting any two points, and the intersection of any two planes represents a line in space. As the UAV should fly among obstacles, reliability in collision avoidance is the foremost priority. Thus, the problem of collision avoidance with a suitable performance index will form an optimal control problem (OCP) for which only a feasible solution is sought. However, for comparison, the optimal solution will also be calculated using a custom solver. The optimal control problem consists of the following.

\subsubsection{Cost Function}

The performance index for maintaining close distance to the desired flight path along with the reliability in the avoidance of obstacles are now defined in as the cost function. Let $y=0$ and $z=z_{d}$ be two planes whose intersection determines a desired path (a straight line), where $t \in\left[t_{0}, t_{f}\right]$. Then, the expression $\left[y(t)^{2}+\left(z(t)-z_{d}\right)^{2}\right]$ represents the square of the deviation of the flight trajectory from the desired path. For obstacle avoidance, a robustness function similar to that in [25] is adopted, that is,

$$
r(X(\cdot))=\sum_{j=1}^{n}\left[e^{e^{\left(-h_{j}(x, y, z)\right)}}-1\right]
$$

where the robustness function is defined such that the value of the function becomes infinite at the center of an obstacle, thereby a UAV never goes to the center, $n$ is the number of obstacles, and $h_{j}(x, y, z)$ defines the obstacle shape, size, and location as in ([4], Chapter 4), that is,

$$
h_{j}(x, y, z)=\ln \left[\left|\left(\frac{x-x_{c j(t)}}{a_{j}}\right)\right|^{p_{x_{j}}}+\left|\left(\frac{y-y_{c j(t)}}{b_{j}}\right)\right|^{p_{y_{j}}}+\left|\left(\frac{z-z_{c j(t)}}{c_{j}}\right)\right|^{p_{z_{j}}}\right] \geq \epsilon_{s}>0
$$

where the inequality represents the obstacle avoidance condition, and the terms inside the logarithm should amount to be greater than one. In the inequality, the subscript $c j(t)$ 
represents the location of the center of an obstacle $j$ at time $t$. In the case of a moving obstacle, the location of its center changes. In the obstacle constraint, $\left\{p_{x_{j}}, p_{y_{j}}, p_{z_{j}}\right\} \geq 2$ are indexes associated with the axes $x, y, z$, respectively, and $\left\{a_{j}, b_{j}, c_{j}\right\}$ refers to the distance of the obstacle boundary from its center position $\left(x_{c j(t)}, y_{c j(t)}, z_{c j(t)}\right)$. If $\left\{p_{x_{j}}, p_{y_{j}}, p_{z_{j}}\right\}=2$ and $\left(a_{j}=b_{j}=c_{j}\right)$, then the inequality (3.3) represents the avoidance condition around a sphere, and if $\left\{p_{x_{j}}, p_{y_{j}}, p_{z_{j}}\right\} \rightarrow \infty$ with $\left(a_{j}=b_{j}=c_{j}\right)$, then the inequality is the avoidance condition around a cube-shaped obstacle. The constant $\epsilon_{s}$ is used for efficient numerical calculations and is such that $0<\epsilon_{s}<<1$.

The overall objective function is completed by adding penalty terms to each objective, as follows:

$$
J(X(\cdot), U(\cdot))=\int_{t_{0}}^{t_{f}}\left[\beta y(t)^{2}+\eta\left(z(t)-z_{d}\right)^{2}+r(X(\cdot))\right] d t
$$

where the penalty terms $\beta>0$ and $\eta>0$ are associated with the planes $y=0$ and $z=z_{d}$, respectively.

\subsubsection{Constraints}

To solve the computational problem, upper and lower limits (resp., $\overline{(\cdot)}$ and $\underline{(\cdot)}$ ) are defined on the states and the saturation in controls. There are other constraints as well: the obstacle avoidance constraints (3.3) and the state constraints (endpoint position constraints) at time $t_{0}$ and $t_{f}$. The state conditions at time $t_{0}$ and $t_{f}$ are

$$
\begin{aligned}
x\left(t_{0}\right) & =x_{0} \\
y\left(t_{0}\right) & =y_{0} \\
z\left(t_{0}\right) & =z_{0} \\
v\left(t_{0}\right) & =v_{0} \\
\gamma\left(t_{0}\right) & =\gamma_{0} \\
\psi\left(t_{0}\right) & =\psi_{0}
\end{aligned}
$$

where $x_{0}, y_{0}$, and $z_{0}$ refer to the position of the UAV at time $t_{0}$.

$$
\begin{aligned}
& x\left(t_{f}\right)=x_{f} \\
& y\left(t_{f}\right)=y_{f} \\
& z\left(t_{f}\right)=z_{f}
\end{aligned}
$$


where $x_{f}, y_{f}$, and $z_{f}$ refer to the position of the UAV at time $t_{f}$.

\subsubsection{Problem Definition}

The following model trajectory generation problem is considered: given an initial condition (3.5) and the final condition (3.6), find the control-state pair $(X(t), U(t))$ that minimizes the cost (3.4) subject to

dynamic equations $(3.1)+$

constraints (3.3), (3.5) and (3.6)

$$
\begin{gathered}
X=(x, y, z, v, \psi, \gamma) \\
U=(\alpha, \phi, T)
\end{gathered}
$$

The optimal control problem summarized in (3.7) is the one that we would ultimately like an optimal solution for. However, as stated in the introduction, the direct solution of (3.7) may be computationally expensive, in particular due to a lack of a good feasible initial guess. In what follows, a method is presented to rapidly generate a feasible initial guess for (3.7). This feasible initial guess may either be used as the flight trajectory in its own right, or may be used as initial guess to solve (3.7). The benefits of both the possibilities shall be demonstrated in Section 4.4.

A two-stage approach is followed. In the first step, in Section 3.2.4, the optimal control problem in (3.7), which uses a dynamic problem, is transformed to an optimal control problem with a simplified kinematic model, while preserving the bounds on the control constraints of the dynamic model. Then, in the second step, in Section 3.3, the optimal control problem with the new kinematic model is then transformed into a simplified geometric problem with fewer optimization parameters, which will be used to obtain the feasible initial guess. Section 3.3.3 details how to recover the feasible initial guess to problem (3.7) from the solution of the simplified geometric problem. Finally, the approach outlined in Chapter 2 will be used to rapidly and reliably solve the geometric problem. 


\subsubsection{Simplification of the System Dynamic Equation}

The problem (3.7) is a nonlinear optimal control problem, and a method for rapid feasible solution generation will now be provided. To this end, the last three equations of the dynamic equation (3.1) are simplified, and this simpler system will still produce a feasible solution to the dynamic model.

In Equation (3.1), in the expressions for $\dot{v}, \dot{\gamma}$, and $\dot{\psi}$, the control input $\alpha$ affects the lift, the drag, and the thrust direction. The stall angle of the wing determines the effectiveness of the $\alpha$ angle, beyond which the wing loses its lift. Here, an assumption is made that the range of the angle of attack $(\alpha)$ lies in a small angle region such that $\sin ( \pm \alpha) \approx \pm \alpha$ and $\cos ( \pm \alpha) \approx 1$. This leads to the following expressions for $\dot{v}, \dot{\gamma}$ and $\dot{\psi}$ :

$$
\begin{aligned}
\dot{v} & =\frac{1}{M}\left[-\frac{1}{2} \rho v^{2} S\left(C_{D_{0}}+K C_{L_{\alpha}}^{2} \alpha^{2}\right)+T \cos (\alpha)-M g \sin (\gamma)\right] \\
& \approx \frac{1}{M}\left[-\frac{1}{2} \rho v^{2} S\left(C_{D_{0}}+K C_{L_{\alpha}}^{2} \alpha^{2}\right)+T-M g \sin (\gamma)\right] \\
\dot{\gamma} & =\frac{1}{M v}\left[\left(\frac{1}{2} \rho v^{2} S C_{L_{\alpha}} \alpha\right) \cos (\phi)+T \sin (\alpha) \cos (\phi)-M g \cos (\gamma)\right] \\
\approx & \frac{1}{M v}\left[\left(\frac{1}{2} \rho v^{2} S C_{L_{\alpha}} \alpha\right) \cos (\phi)+T \alpha \cos (\phi)-M g \cos (\gamma)\right] \\
\dot{\psi} & =\frac{1}{M v \cos (\gamma)}\left[\left(\frac{1}{2} \rho v^{2} S C_{L_{\alpha}} \alpha\right) \sin (\phi)+T \sin (\alpha) \sin (\phi)\right] \\
& \approx \frac{1}{M v \cos (\gamma)}\left[\left(\frac{1}{2} \rho v^{2} S C_{L_{\alpha}} \alpha\right) \sin (\phi)+T \alpha \sin (\phi)\right]
\end{aligned}
$$

To simplify further, a new set of controls $U_{s}=\left(a, \omega_{\gamma}, \omega_{\psi}\right)$ is defined: acceleration $(a)$, rate of change of the flight path angle $\left(\omega_{\gamma}\right)$, and rate of change of the heading angle $\left(\omega_{\psi}\right)$. That is, 


$$
\begin{aligned}
a & =\frac{1}{M}\left[-\frac{1}{2} \rho v^{2} S\left(C_{D_{0}}+K C_{L_{\alpha}}^{2} \alpha^{2}\right)+T-M g \sin (\gamma)\right] \\
\dot{v} & =a \\
\omega_{\gamma} & =\frac{1}{M v}\left[\left(\frac{1}{2} \rho v^{2} S C_{L_{\alpha}} \alpha\right) \cos (\phi)+T \alpha \cos (\phi)-M g \cos (\gamma)\right] \\
\dot{\gamma} & =\omega_{\gamma} \\
\omega_{\psi} & =\frac{1}{M v \cos (\gamma)}\left[\left(\frac{1}{2} \rho v^{2} S C_{L_{\alpha}} \alpha\right) \sin (\phi)+T \alpha \sin (\phi)\right] \\
\dot{\psi} & =\omega_{\psi}
\end{aligned}
$$

Conversely, from $\left(a, \omega_{\gamma}, \omega_{\psi}\right)$, the expression for $\alpha, \phi, T$ can be obtained using (3.11)(3.13), which are the controls to the dynamic equation (3.1), as follows:

$$
\begin{gathered}
T=M a+\frac{1}{2} \rho v^{2} S\left(C_{D_{0}}+K C_{L_{\alpha}}^{2} \alpha^{2}\right)+M g \sin (\gamma) \\
\phi=\arctan \left(\frac{v \cos (\gamma) \omega_{\psi}}{v \omega_{\gamma}+g \cos (\gamma)}\right) \\
\alpha=\left(\frac{M v \omega_{\gamma}+M g \cos (\gamma)}{\cos (\phi)\left(\frac{1}{2} \rho v^{2} S C_{L_{\alpha}}+T\right)}\right)
\end{gathered}
$$

Given the constraints for the controls $(\alpha: \underline{\alpha} \leq \alpha \leq \bar{\alpha}, \phi: \underline{\phi} \leq \phi \leq \bar{\phi}, T: \underline{T} \leq T \leq$ $\bar{T})$, the limits on $U_{s}=\left(a, \omega_{\gamma}, \omega_{\psi}\right)$ are obtained as follows. The derivation is shown in Appendix 2. Assuming that the mass $(M)$ is constant for a small amount of time, and at any given instant the values of state parameters $(X)$ are known, then the rate of change of the states $\dot{v}, \dot{\psi}, \dot{\gamma}$ can be calculated along with their possible upper and lower limits at that instant using Equations (3.8)-(3.10). These are reasonable assumptions, as the change in mass is negligible for a short period of time. As the states are $X=(x, y, z, v, \gamma, \psi)$, 
the limits for the controls $U_{s}$ can be written as

Given the bounds on the controls $(\alpha, \phi, T)$, the bounds on $U_{s}=\left(a, \omega_{\gamma}, \omega_{\psi}\right)$ are obtained so that (3.14), (3.15), and (3.16) are satisfied.

Assumptions:

1. $\underline{T} \geq 0 N, \underline{\phi}=-\bar{\phi}, 0<\bar{\phi}<\frac{\pi}{2} \mathrm{rad}$, and $\bar{\alpha}>0 \mathrm{rad}$.

2. $v>0 \mathrm{~m} / \mathrm{s}, \cos \gamma>0 \Longrightarrow\left(|\gamma|<\frac{\pi}{2} \mathrm{rad}\right.$ ), and $\cos \phi>0$ (by assumption 1).

\section{- Bounds on a:}

$$
\begin{gathered}
\underline{a}=\left\{\begin{array}{cc}
\frac{1}{M}\left[\underline{T}-\frac{1}{2} \rho v^{2} S C_{D_{0}}-M g \sin (\gamma)\right], & \underline{\alpha} \leq 0 \\
\frac{1}{M}\left[\underline{T}-\frac{1}{2} \rho v^{2} S\left(C_{D_{0}}+K C_{L_{\alpha}}^{2} \underline{\alpha}^{2}\right)-M g \sin (\gamma)\right] & \underline{\alpha}>0
\end{array}\right. \\
\bar{a}=\frac{1}{M}\left[\bar{T}-\frac{1}{2} \rho v^{2} S\left(C_{D_{0}}+K C_{L_{\alpha}}^{2} \bar{\alpha}^{2}\right)-M g \sin (\gamma)\right] .
\end{gathered}
$$

These ensure that $\underline{a} \leq a \leq \bar{a} \Longrightarrow \underline{T} \leq T \leq \bar{T}$

\section{- Bounds on $\omega_{\gamma}$ :}

For $\underline{\omega}_{\gamma}$, by assumption 1, it is required that $|\phi| \leq \frac{\pi}{2}$ rad. Therefore, by (3.15), it is required that $\omega_{\gamma}>-\frac{g \cos (\gamma)}{v}$, and this is satisfied if $\underline{\omega_{\gamma}}=-\frac{g \cos (\gamma)}{v}+\epsilon_{c}$ for any $\epsilon_{c}>0$. Expressions for the bounds on $\omega_{\gamma}$ and $\omega_{\psi}$ can be similarly obtained.

$$
\begin{gathered}
\underline{\omega_{\gamma}}=\left\{\begin{array}{cc}
-\frac{g \cos (\gamma)}{v}+\epsilon_{c}, & \underline{\alpha} \leq 0 \\
\frac{\alpha\left(\frac{1}{2} \rho v^{2} S C_{L_{\alpha}}+\bar{T}\right)}{M v}-\frac{g \cos (\gamma)}{v} & \underline{\alpha}>0
\end{array} \text { where } \epsilon_{c}>0\right. \\
\bar{\omega}_{\gamma}=\frac{\bar{\alpha} \cos (\bar{\phi})\left(\frac{1}{2} \rho v^{2} S C_{L_{\alpha}}+\underline{T}\right)-M g \cos (\gamma)}{M v}
\end{gathered}
$$

These ensure that $\underline{\omega_{\gamma}} \leq \omega_{\gamma} \leq \overline{\omega_{\gamma}} \Longrightarrow \underline{\alpha} \leq \alpha \leq \bar{\alpha}$.

Considering the case $\underline{\omega_{\gamma}}$ when $\underline{\alpha}>0$, by (3.16), it should hold that $\underline{\alpha} \leq \frac{M v \omega_{\gamma}+M g \cos (\gamma)}{A}$, where $\left.A=\cos (\phi) \overline{\left(\frac{1}{2} \rho\right.} v^{2} S C_{l_{\alpha}}+T\right)>0$ by assumptions 1 and 2 . Therefore, it should hold that $\frac{\underline{\alpha} A-M g \cos (\gamma)}{M v} \leq \omega_{\gamma}$. 


$$
\begin{gathered}
\text { Since, } \quad \frac{\underline{\alpha} A-M g \cos (\gamma)}{M v} \leq \frac{\underline{\alpha}\left(\frac{1}{2} \rho v^{2} S C_{L_{\alpha}}+\bar{T}\right)-M g \cos (\gamma)}{M v}=\underline{\omega_{\gamma}} \leq \omega_{\gamma} \\
\Longrightarrow \underline{\alpha} \leq \frac{M v \omega_{\gamma}+M g \cos (\gamma)}{A}=\alpha \text {, as required. }
\end{gathered}
$$

\section{- Bounds on $\omega_{\psi}$ :}

$$
\begin{aligned}
\underline{\omega_{\psi}} & =\frac{\tan (\underline{\phi})\left(v \underline{\omega_{\gamma}}+g \cos (\gamma)\right)}{v \cos (\gamma)} \\
\bar{\omega}_{\psi} & =\frac{\tan (\bar{\phi})\left(v \underline{\omega_{\gamma}}+g \cos (\gamma)\right)}{v \cos (\gamma)}
\end{aligned}
$$

Note: By assumption 1, $\underline{\phi}=-\bar{\phi}$; thus, $\underline{\omega_{\psi}}=-\bar{\omega}_{\psi}$, which is correct. These ensure that $\underline{\omega_{\psi}} \leq \omega_{\psi} \leq \overline{\omega_{\psi}} \Longrightarrow \underline{\phi} \leq \phi \leq \bar{\phi}$, which may be proved as in the case of $\omega_{\gamma}$ above.

Thus, the simplified system equation for a fixed-wing UAV can be written as in [91]

$$
\left[\begin{array}{c}
\dot{x} \\
\dot{y} \\
\dot{z} \\
\dot{v} \\
\dot{\gamma} \\
\dot{\psi}
\end{array}\right]=\left[\begin{array}{c}
v \cos \gamma \cos \psi \\
v \cos \gamma \sin \psi \\
v \sin \gamma \\
a \\
\omega_{\gamma} \\
\omega_{\psi}
\end{array}\right]
$$

The limits on the new control parameters are

$$
U_{s}=\left\{\begin{array}{cc}
a: & \underline{a} \leq a \leq \bar{a} \\
\omega_{\gamma}: & \underline{\omega_{\gamma}} \leq \omega_{\gamma} \leq \overline{\omega_{\gamma}} \\
\omega_{\psi}: & \underline{\omega_{\psi}} \leq \omega_{\psi} \leq \overline{\omega_{\psi}}
\end{array}\right\} \subset \mathbb{R}^{3}
$$

where the expressions for the bounds can be deduced from (3.17)-(3.22).

The problem formulation with the simplified equation (3.23) that yields an optimal trajectory for the original problem is the following, given an initial condition (3.5) and the final condition (3.6), find the control-state pair $\left(X(t), U_{s}(t)\right)$ that minimizes the cost (3.4) subject to 


$$
\begin{gathered}
\text { dynamic equations }(3.23)+ \\
\text { constraints }(3.3) \text {, and }(3.24) \\
X=(x, y, z, v, \psi, \gamma) \\
U_{s}=\left(a, \omega_{\gamma}, \omega_{\psi}\right)
\end{gathered}
$$

Once problem (3.25) has been solved, a set of optimal controls $U_{s}\left(a, \omega_{\gamma}\right.$ and $\left.\omega_{\psi}\right)$ is obtained. Using Equations (3.14)-(3.16), the set of controls $\alpha, \phi$, and $T$ is obtained for the problem (3.7). Henceforth, Equation (3.23) will be used as a fixed-wing UAV system equation, with its control bounds (3.24) for trajectory generation. Although, there is no guarantee that the solution to (3.25) is locally optimal for (3.7), but instead we get a suboptimal feasible solution, extensive numerical examples show that the solution to (3.25) yields cost very similar to the solution to (3.7) (see Section 3.4.3).

As stated in the Introduction of this chapter, rapid optimal trajectory generation may be computationally intensive owing to the nature of nonlinear programming (NLP) algorithms. Nevertheless, a feasible trajectory for the problem is also a challenge, as it cannot be easily obtained by simple expressions. The nature of the constraints (stationary and moving obstacles) and the objective of the problem are such that the use of an iterative algorithm is inevitable (because the initial point is unknown). However, to reduce computation time or to increase the rate of feasible solution generation, the following methods are used.

1. The problem (3.25) is transformed to an optimization problem whose parameters are the location of the points in $3 D$ space $(x, y, z)$ and the associated velocity $(v)$. Thereby, the number of optimization parameters is reduced. Furthermore, since $(v)$ is an optimization parameter, dynamic elements such as moving obstacles can be handled.

2. The resulting problem is nonlinear programming (NLP), and thus it cannot be rapidly solved. Therefore, a convex approximation is performed, and a convex solver is then used. Subsequently, re-convexification is performed at the new solution point, and thus a feasible solution is obtained. As the convex solver is fast, with an approximately known computation time and number of iterations [62], it is suitable for rapid feasible solution generation. 
3. To solve convex problems rapidly, a custom quadratic programming (QP) solver is developed. It involves only four types of mathematical operators $\left(+,-,{ }^{*}, /\right)$ and reduces programming overhead by eliminating the use of external libraries, including computation with zeros. This increases computation speed approximately $0 \sim 50$ times depending on the size of the problem on laptop computers (compared to general solvers).

\subsection{An Approach for a Feasible Trajectory Genera- tion}

A feasible trajectory for a fixed-wing UAV in a cluttered environment is one that the UAV can fly safely among obstacles utilizing controls within saturation limits. However, it is not necessarily optimal with respect to the given cost function. In order to do so, $N$ points are introduced between an initial and the goal point in the $x-y-z$ space so that the initial and the goal point of a UAV are $\left(x_{0}, y_{0}, z_{0}\right)$ and $\left(x_{N+1}, y_{N+1}, z_{N+1}\right)$, respectively. The link between the initial and the goal point connected by the set of the $N$ intermediate points should be determined to obtained a flyable trajectory (with obstacle avoidance). In order that the points arranged in the space be a feasible path, they should satisfy the UAV's state and control constraints. Thus, in the following, expressions are developed that define the relationships between points in space and the UAV's state and control constraints. Figure 3.1 shows the distribution of $N$ points between an initial and the goal point.

To relate the kinematic equation (3.23) of a fixed-wing UAV to the points in $x-y-z$ space, it is assumed that the UAV moves in a straight line between two consecutive free points. In Figure 3.1, $\left(x_{0}, y_{0}, z_{0}\right)$ is an initial point, $\psi_{0}$ is an initial heading angle, and $\gamma_{0}$ is an initial flight path angle. Similarly, $\left(x_{N}, y_{N}, z_{N}\right)$ is the free point before $\left(x_{N+1}, y_{N+1}, z_{N+1}\right)$, where $\psi_{N}$ and $\gamma_{N}$ are the heading angle and the flight path angle between the points $N$ and $N+1$, respectively. It is assumed the direction of the heading angle and the flight path angle at the points $N$ and $N+1$ are the same, as the direction is measured between the points $N$ and $N+1$. In the following, the detailed procedure will be presented in which a trajectory generation problem is stated so that the UAV's constraints (for example, bounds on thrust and bank angle), obstacle constraints, and 


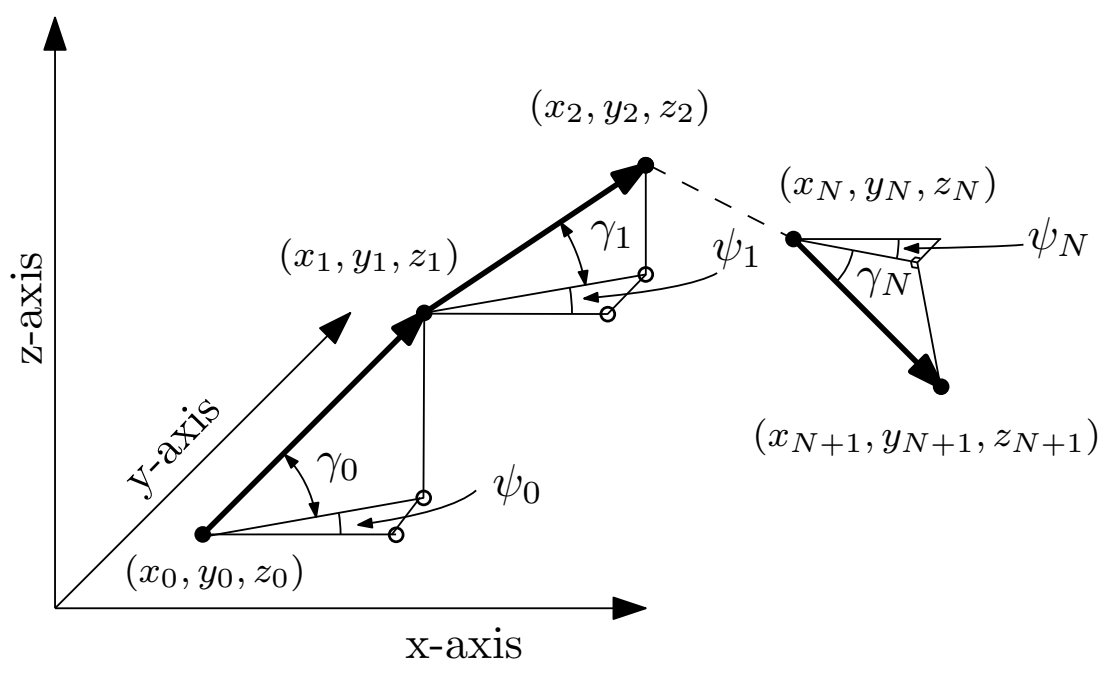

Figure 3.1: $N$ discrete points in 3D space.

end-point conditions are preserved.

Let $\left(x_{i}, y_{i}, z_{i}\right)$ represent the location of the $i^{\text {th }}$ point in $x-y-z$ space, let $v_{i}$ be the velocity at that point, and it is assumed that the velocity between the points $i$ and $i+1$ is the average of $v_{i}$ and $v_{i+1} . v_{i}$ represents UAV's velocity at $i$. Accordingly, the acceleration profile between the points is constant. $\delta s_{i}$ is defined to be the displacement covered in time $\delta t_{i}$ in 3D space, so that $\delta s_{i}$ and $\delta t_{i}$ are given by

$$
\begin{gathered}
\delta s_{i}=\sqrt{\delta x_{i}^{2}+\delta y_{i}^{2}+\delta z_{i}^{2}} \\
\delta t_{i}=\frac{2 \delta s_{i}}{v_{i}+v_{i-1}}
\end{gathered}
$$

where $\delta x_{i}=x_{i}-x_{i-1}, \delta y_{i}=y_{i}-y_{i-1}$, and $\delta z_{i}=z_{i}-z_{i-1}(i=1, \ldots, N+1)$ are the displacements between two consecutive points in the $x, y$, and $z$ axes, respectively, at the $i^{t h}$ instant, and $\delta t_{i}$ is the time between two consecutive points, which is measured by the average velocity of consecutive points.

As $\psi$ is the heading angle measured from positive the $x$-axis to the positive $y$-axis, and $\gamma$ is the flight path angle measured from the $x-y$ plane to the flight path, the expression for $\psi_{i}$ and $\gamma_{i}$ between $(i-1)^{t h}$ and $i^{t h}$ can be written as 


$$
\begin{gathered}
\psi_{i}=\arctan \left(\frac{\delta y_{i}}{\delta x_{i}}\right) \\
\gamma_{i}=\arctan \left(\frac{\delta z_{i}}{\sqrt{\delta x_{i}^{2}+\delta y_{i}^{2}}}\right)
\end{gathered}
$$

where $i=1, \ldots, N+1, \psi_{N+1}=\psi_{N}$, and $\gamma_{N+1}=\gamma_{N}$

The control constraints in (3.24) are transformed as follows:

- For $\dot{v}=a$, using the approximation $\dot{v} \approx \frac{\delta v}{\delta t}$, one has $\frac{\delta v}{\delta t}=a$. Using $\delta v=v_{i}-v_{i-1}$, the average acceleration between two consecutive points becomes $\frac{v_{i}-v_{i-1}}{\delta t_{i}}=\frac{v_{i}^{2}-v_{i-1}^{2}}{2 \delta s_{i}}=a$. Thus,

$$
a=\frac{v_{i}^{2}-v_{i-1}^{2}}{2 \delta s_{i}}
$$

- For $\dot{\gamma}=\omega_{\gamma}$, one has $\frac{\delta \gamma}{\delta t}=\omega_{\gamma}$. Using $\delta \gamma=\gamma_{i}-\gamma_{i-1}$, the average rate of change of the flight path angle between two consecutive points becomes $\frac{\gamma_{i}-\gamma_{i-1}}{\delta t_{i}}=$ $\frac{\left(\gamma_{i}-\gamma_{i-1}\right)\left(v_{i}+v_{i-1}\right)}{2 \delta s_{i}}=\omega_{\gamma}$. Thus,

$$
\omega_{\gamma}=\frac{\left(\gamma_{i}-\gamma_{i-1}\right)\left(v_{i}+v_{i-1}\right)}{2 \delta s_{i}}
$$

- Similarly, for $\dot{\psi}=\omega_{\psi}$, one has $\frac{\delta \psi}{\delta t}=\omega_{\psi}$. Using $\delta \psi=\psi_{i}-\psi_{i-1}$, the average rate of change of the heading angle between two consecutive points becomes $\frac{\psi_{i}-\psi_{i-1}}{\delta t_{i}}=$ $\frac{\left(\psi_{i}-\psi_{i-1}\right)\left(v_{i}+v_{i-1}\right)}{2 \delta s_{i}}=\omega_{\psi}$. Thus,

$$
\omega_{\psi}=\frac{\left(\psi_{i}-\psi_{i-1}\right)\left(v_{i}+v_{i-1}\right)}{2 \delta s_{i}}
$$

\subsubsection{Cost Function}

As the desired path is given by the intersection of two planes, $y=y_{a}, z=z_{a}$, it may be assumed that $y_{a}=z_{a}=0$ for simplicity. Then, the cost function is defined to arrange $N+2$ points in space ( $N$ free points, and two fixed endpoints) so that they may remain close to the intersection of the planes $y=0, z=0$. A cost function is added to minimize 
the sum of the squares of the distances between adjacent points so that equidistant point distributions are obtained, but this also leads to the shortest path and limits the path curvature (the path should be as straight as possible). Finally, the robustness function in (3.2) is added to the cost function to obtain

$$
\begin{aligned}
J_{G}(\cdot)= & \sum_{i=1}^{N}\left[\beta y_{i}^{2}+\eta z_{i}^{2}+r\left(x_{i}, y_{i}, z_{i}\right)\right] \\
& +\kappa \sum_{i=1}^{N+1}\left[\left(x_{i}-x_{i-1}\right)^{2}+\left(y_{i}-y_{i-1}\right)^{2}+\left(z_{i}-z_{i-1}\right)^{2}\right] \\
\text { where, } \quad & r\left(x_{i}, y_{i}, z_{i}\right)=\sum_{j=1}^{n}\left[e^{\left.e^{\left(-h_{j}\left(x_{i}, y_{i}, z_{i}\right)\right)}-1\right]}\right.
\end{aligned}
$$

where $\kappa>0 . \beta>0$, and $\eta>0$ are penalty parameters associated with the planes $y=0$ and $z=0$, respectively. $\left(x_{i}, y_{i}, z_{i}\right)$ are the coordinates of free points, where $i=1 \ldots N$. The terms $\eta z_{i}^{2}$ and $\beta y_{i}^{2}$ locate the free points that are close to the line represented by the intersection of the planes $y=0, z=0$.

\subsubsection{Constraints}

\section{Equality Constraint}

In the cost function (3.33), a term was added to minimize the sum of the squares of the distances between adjacent points. The rationale behind that was to have a sequential point distribution (equidistant). Although the addition of other terms in the cost function does not ensure equidistant adjacent points, it serves the purpose of minimizing the relative cost owing to that term. However, to obtain a sequential point distribution between two points, it is added as a constraint that the distances between consecutive points should be equal. The reason for this equality constraint is that it helps finding a good starting point for the custom solver iterations. It follows from [65] Section 5.3 that the initialization process requires an equality constraint along with other Karush-KuhnTucker conditions to find a good starting point irrespective of the user-supplied initial guess. Therefore, the constraint is added as follows. Let $d_{i}$ be the distance between the points $i-1$ and $i$, and $d_{i+1}$ be the distance between the points $i$ and $i+1$, then

$$
d_{i}=d_{i+1}
$$

which upon squaring both the sides yields, 


$$
\begin{gathered}
\left(x_{i+1}-x_{i}\right)^{2}+\left(y_{i+1}-y_{i}\right)^{2}+\left(z_{i+1}-z_{i}\right)^{2}=\left(x_{i}-x_{i-1}\right)^{2}+\left(y_{i}-y_{i-1}\right)^{2}+\left(z_{i}-z_{i-1}\right)^{2} \\
\forall i=1, \ldots, N
\end{gathered}
$$

\section{Obstacle Constraint}

The obstacle constraint defined in (3.3) is used as it is, where $\left(x_{i}, y_{i}, z_{i}\right)$ is the location of points in space. As the obstacle avoidance criteria are functions of the position of the UAV and the location of the center of an obstacle in $x y z$, the equation can be directly added into the problem definition, as follows:

$$
h_{j}(x, y, z)=\ln \left[\left|\left(\frac{x_{i}-x_{c j(t)}}{a_{j}}\right)\right|^{p_{x_{j}}}+\left|\left(\frac{y_{i}-y_{c j(t)}}{b_{j}}\right)\right|^{p_{y_{j}}}+\left|\left(\frac{z_{i}-z_{c j(t)}}{c_{j}}\right)\right|^{p_{z_{j}}}\right] \geq \epsilon_{s}>0
$$

where the parameters are as in (3.3).

\section{State Constraint}

The state constraints for the problem (3.7) are transformed directly to the problem defined above with the current formulation, as follows:

$$
\begin{aligned}
x_{i}: & \underline{x} \leq x_{i} \leq \bar{x} \\
y_{i}: & \underline{y} \leq y_{i} \leq \bar{y} \\
z_{i}: & \underline{z} \leq z_{i} \leq \bar{z} \\
v_{i}: & \underline{v} \leq v_{i} \leq \bar{v} \\
\gamma_{i}: & \underline{\gamma} \leq \gamma_{i} \leq \bar{\gamma} \\
\psi_{i}: & \underline{\psi} \leq \psi_{i} \leq \bar{\psi}
\end{aligned}
$$

where $\psi_{i}$ and $\gamma_{i}$ are defined as in (3.28) and (3.29), respectively.

\section{Control Constraint}

The control constraints from (3.24) is transformed as follows:

- By (3.24), $a$ has bounds $\underline{a} \leq a \leq \bar{a}$. Using $a$ from (3.30), one obtains 


$$
\underline{a} \leq \frac{v_{i}^{2}-v_{i-1}^{2}}{2 \delta s_{i}} \leq \bar{a}
$$

where $\underline{a}$ and $\bar{a}$ are defined in (3.17) and (3.18), respectively.

- Again by (3.24), $\omega_{\gamma}$ has bounds $\underline{\omega_{\gamma}} \leq \omega_{\gamma} \leq \overline{\omega_{\gamma}}$. Using $\omega_{\gamma}$ from (3.31), one obtains

$$
\underline{\omega_{\gamma}} \leq \frac{\left(\gamma_{i}-\gamma_{i-1}\right)\left(v_{i}+v_{i-1}\right)}{2 \delta s_{i}} \leq \overline{\omega_{\gamma}}
$$

where $\underline{\omega_{\gamma}}$ and $\overline{\omega_{\gamma}}$ are defined in (3.19) and (3.20), respectively.

- Similarly, by (3.24), $\omega_{\psi}$ has bounds as, $\underline{\omega_{\psi}} \leq \omega_{\psi} \leq \overline{\omega_{\psi}}$. Using $\omega_{\psi}$ from (3.32) we get

$$
\underline{\omega_{\psi}} \leq \frac{\left(\psi_{i}-\psi_{i-1}\right)\left(v_{i}+v_{i-1}\right)}{2 \delta s_{i}} \leq \overline{\omega_{\psi}}
$$

where $\underline{\omega_{\psi}}$ and $\overline{\omega_{\psi}}$ are defined in (3.21) and (3.22), respectively.

\subsubsection{Problem Definition}

A problem is now defined whose solution provides a feasible set of controls to fly around obstacles along with state and end-point constraints. Given an initial condition $\left(x_{0}, y_{0}, z_{0}, v_{0}, \gamma_{0}, \psi_{0}\right)$, and the final condition $\left(x_{N+1}, y_{N+1}, z_{N+1}\right)$, find the parameters $\left(x_{i}, y_{i}, z_{i}, v_{i} \mid i=1, \ldots, N\right)$ to minimize the cost (3.33) subject to

equality constraint (3.34)

obstacle constraint (3.35)

control constraints (3.37), (3.38), (3.39), and

bounds on optimization variables (3.36)

The problem (3.40) is an nonlinear programming (NLP) problem with $4 N$ variables $\left(x_{i}, y_{i}, z_{i}, v_{i}\right)$. To solve this problem, an initial starting point is required. Given the initial and the goal points, the following is used as a set of starting points for (3.40):

$x_{i}=$ equispaced points from $x_{0}$ to $x_{N+1}$

$y_{i}=$ equispaced points along the desired reference path from $y_{0}$ to $y_{N+1}$

$z_{i}=$ equispaced points along the desired reference path from $z_{0}$ to $z_{N+1}$ 
$v_{i}=$ minimum $v_{i}$ such that $\underline{v} \leq v_{i} \leq \bar{v}, \forall i=1, \ldots, N$.

This initialization implies that the problem may have infeasible constraints. That is, when these starting points are provided without considering obstacle locations, there is a possibility that these points in 3D space may be inside an obstacle, which will cause the obstacle constraint to be infeasible at the very beginning of the solution procedure. However, using the quadratic programming (QP) algorithm from [65], an obstacle-free feasible solution can be obtained, regardless of the solution procedure. As discussed in Section 3.3.2, the answer to this lies in the initialization procedure of the algorithm presented in Section 5.3 of [65], where the equality constraints are solved together with inequality constraints, and then slack variables are introduced so that the inequality constraints are satisfied for the quadratic programming (QP) problem as well. Accordingly, the algorithm solves a feasible quadratic programming (QP) problem at each sequential convex programming (SCP) iteration. In addition to the infeasibility owing to obstacles, infeasibility issues could also arise when the quadratic programming (QP) problem is solved using sequential convex programming (SCP). Specifically, since we deal with the cost function which is not convex (due to the presence of the robustness function (3.2)), its Hessian may not be positive semi-definite, particularly for points near or inside an obstacle boundary. In such case, the quadratic programming (QP) problem is first solved using the method that resembles the infeasible start Newton method ([62], Chapter 10.3) to arrive at a feasible solution free from obstacles, and then the regular procedure as stated in [32] is applied to solve the problem. Thus, strictly speaking, we are not doing sequential convex programming (SCP), because non-convexity of the cost function means that the Hessian is not guaranteed to be convex. However, in all numerical examples tried, the minimum eigenvalue of the Hessian of the overall cost function was very small in magnitude, and the custom convex quadratic programming (QP) solver still worked very well even in worst case scenarios when initial guesses were infeasible and passed through the obstacles (see Section 3.4.6).

Once problem (3.40) has been solved to a required accuracy, a feasible trajectory for the control problem (3.25) can be obtained as follows. To recover the remaining states, Equations (3.26) and (3.27) are first used to compute the time associated with the node points, and then Equations (3.28) and (3.29) are used to compute the heading angle $(\psi)$ and the flight path angle $(\gamma)$, respectively. To recover the controls, Equation (3.30) is used for acceleration $(a),(3.31)$ for $\omega_{\gamma}$, and (3.32) for $\omega_{\psi}$ between consecutive node 
points. Then, the original controls for problem (3.7) are recovered using Equation (3.14) for thrust, (3.15) for $\phi$, and (3.16) for $\alpha$. However, the solutions by (3.14), (3.15), and (3.16) are for small angle approximation to $\alpha$. Therefore, to recover the exact controls when $\alpha$ is not a small angle approximation, the following steps are performed. In the first step, the base values for thrust, $\phi$, and $\alpha$ are obtained using Equations (3.14), (3.15), and (3.16), respectively. Then, in the second step, an iterative approach (such as Newton's Method) over the last three equations of (3.1) is used to obtain the controls for the problem (3.7).

\subsection{Numerical Example}

The UAV parameters for which a trajectory among obstacles will be generated are first introduced. For the numerical test, parameters such as mass, wing surface area, and wingspan of a fixed-wing electric-powered UAV (Ascent [1]) are considered. Table 3.1 shows the UAV parameters. As shown in the table, the mass $(M)$ of the UAV is 0.553 $\mathrm{kg}$, the wing surface area is $0.2135 \mathrm{~m}^{2}$, and the wing span $(b)$ is $1.37 \mathrm{~m}$. Thus, the aspect ratio $(A)$ is 8.79 . In the table, values for the minimum drag coefficient $\left(C_{D_{0}}\right)$, the lift coefficient per angle of attack $C_{L_{\alpha}}$, and the thrust to weight ratio $(T / W)$ are set for the numerical example. To use a reasonable $C_{L_{\alpha}}$ for the UAV, the expression for $C_{L_{\alpha}}$ from ([92], Chapter 3) is used as follows,

$$
C_{L_{\alpha}}=C_{l_{\alpha}} \frac{A}{2+\sqrt{4+A^{2}}}
$$

where $A$ is the Aspect Ratio. Assuming $C_{l_{\alpha}}=2 \pi$ and $A=8.79$ from Table 3.1, we get $C_{L_{\alpha}}=5.01 / \mathrm{rad}$. The thrust to weight ratio $(T / W)$ is set to 1 . Since, $C_{D_{0}}$ is not available, it is assumed to be 0.03 with respect to [1]. Also, the Oswald efficiency $(e)$ for the UAV is not available therefore it is assumed with respect to the results from ([93] for wings with taper ratio of 1 ) as 0.3 . In order to obtain the constant $K$ the expression $K=\frac{1}{\pi A e}$ is used, which gives us its value as $K=0.1207$.

The state and control limits for the model UAV are now defined. Although the $3 D$ space for numerical results could be set as wide as possible, it is constrained for presenting the numerical example, so that the space along the $x$-axis ranges from -1 to $110 \mathrm{~m}$, along 
Table 3.1: Physical and aerodynamic features of the Ascent UAV [1].

\begin{tabular}{lc}
\hline \hline Ascent UAVs parameters & Value \\
\hline Mass $(M)$ & $0.553 \mathrm{~kg}$ \\
Density of Air $(\rho)$ & $1.225 \mathrm{~kg} / \mathrm{m}^{3}$ \\
Wing Surface Area $(S)$ & $0.2135 \mathrm{~m}^{2}$ \\
Acceleration due to gravity $(g)$ & $9.81 \mathrm{~m} / \mathrm{s}^{2}$ \\
Minimum Drag Coefficient $\left(C_{D_{0}}\right)$ & 0.03 (assumed from $[1])$ \\
Wing Span $(b)$ & $1.37 \mathrm{~m}$ \\
Aspect Ratio $(A)$ & $\frac{b^{2}}{S}=8.79$ \\
Oswald Efficiency $(e)$ & 0.3 (assumed from $[93])$ \\
Constant $(K)$ & $\frac{1}{\pi A e}=0.1207$ \\
$C_{L_{\alpha}}$ & $5.01 / \mathrm{rad}$ \\
Thrust to weight ratio $\left(\frac{T}{W}\right)$ & 1 (assumed) \\
\hline \hline
\end{tabular}

the $y$-axis ranges from -50 to $50 \mathrm{~m}$, and along the $z$-axis ranges from 0 to $50 \mathrm{~m}(z=0$ $\mathrm{m}$ is the ground). The bounds (minimum and maximum) on the flight path angle $(\gamma)$ are set to $\pm \frac{\pi}{4} \mathrm{rad}$, and the bounds on the heading angle $(\psi)$ are set to $\pm \pi \mathrm{rad}$. The minimum velocity of the UAV is set to $8 \mathrm{~m} / \mathrm{s}$, and the maximum velocity to $12 \mathrm{~m} / \mathrm{s}$. All these state limits are shown in Table 3.2.

Table 3.2: State constraints for the Ascent UAV

\begin{tabular}{ccc}
\hline \hline State & Lower Bound (unit) & Upper Bound (unit) \\
\hline & & \\
$x$ & $-1 \mathrm{~m}$ & $110 \mathrm{~m}$ \\
$y$ & $-50 \mathrm{~m}$ & $50 \mathrm{~m}$ \\
$z$ & $0 \mathrm{~m}$ & $50 \mathrm{~m}$ \\
$\gamma$ & $-\frac{\pi}{4} \mathrm{rad}$ & $\frac{\pi}{4} \mathrm{rad}$ \\
$\psi$ & $-\pi \mathrm{rad}$ & $\pi \mathrm{rad}$ \\
$v$ & $8 \mathrm{~m} / \mathrm{s}$ & $12 \mathrm{~m} / \mathrm{s}$ \\
\hline \hline
\end{tabular}

The maximum value for the angle of attack was set to $\bar{\alpha}=\frac{10 \pi}{180} \mathrm{rad}$, and the minimum to $\underline{\alpha}=0 \mathrm{rad}$. The maximum and the minimum values for the roll angle were set to $\frac{\pi}{4} \mathrm{rad}$ and $-\frac{\pi}{4} \mathrm{rad}$, respectively. As the UAV was electric-powered, the minimum thrust $\underline{T}$ was set to $0 \mathrm{~N}$. To define the maximum thrust, the load factor $T / W$ was used. As $T / W=1$, we get $T=W=M g$, which in terms of force yields $T=1 \cdot 0.553 \cdot 9.81=5.425 \mathrm{~N}$. Table 3.3 shows the limits on the controls. 
Table 3.3: Control constraints for the Ascent UAV

\begin{tabular}{ccc}
\hline \hline Control & Lower Bound (unit) & Upper Bound (unit) \\
\hline & & \\
$\alpha$ & $0 \mathrm{rad}$ & {$\left[\frac{10 \pi}{180}\right] \mathrm{rad}$} \\
$\phi$ & {$\left[-\frac{\pi}{4}\right] \mathrm{rad}$} & {$\left[\frac{\pi}{4}\right] \mathrm{rad}$} \\
$T$ & $0 \mathrm{~N}$ & $5.425 \mathrm{~N}$ \\
\hline \hline
\end{tabular}

As the simplified model is used, the state and control limits should be defined according to (3.23). The states and the state limits for the problems (3.7) and (3.25) are the same. Therefore, only the control limits (3.24) for the problem (3.25) are presented in Table 3.4, which comes from Section 3.2.4 along with control limits from Table 3.3.

Table 3.4: Control constraints (3.24) for the simplified model (3.23)

\begin{tabular}{ccc}
\hline \hline Parameters & Lower Bound & Upper Bound \\
\hline$a\left(\mathrm{~m} / \mathrm{s}^{2}\right)$ & $-\frac{1}{M}\left[\frac{1}{2} \rho v^{2} S C_{D_{0}}+M g \sin (\gamma)\right]$ & $\frac{1}{M}\left[\bar{T}-\frac{1}{2} \rho v^{2} S\left(C_{D_{0}}+K C_{L_{\alpha}}^{2} \bar{\alpha}^{2}\right)-M g \sin (\gamma)\right]$ \\
$\omega_{\gamma}(\mathrm{rad} / \mathrm{s})$ & {$\left[-\frac{g \cos (\gamma)}{v}+\epsilon_{c}\right]$} & {$\left[\frac{3 \pi \rho v S C_{L_{\alpha}}}{72 \sqrt{2} M}-\frac{g \cos (\gamma)}{v}\right]$} \\
$\omega_{\psi}(\mathrm{rad} / \mathrm{s})$ & {$\left[-\left(\epsilon_{c}\right) /(\cos (\gamma))\right]$} & {$\left[\left(\epsilon_{c}\right) /(\cos (\gamma))\right]$} \\
\hline \hline
\end{tabular}

\subsubsection{Problem Scenario}

A Monte-Carlo test with various obstacle configurations will be performed, and detailed results will be presented for an example case. To demonstrate the benefit of the proposed approach, UAV trajectories will be generated by three different approaches. First, the original problem in (3.25) is solved directly, without finding a good feasible initial guess first. Second, the simplified problem in (3.40) will be solved to obtain a feasible trajectory, and third, again the original problem in (3.25) is solved, but this time first solving (3.40) to obtain a feasible initial guess for (3.25). Then, the methods are compared in terms of computational speed, number of iterations, and cost. Since the aim of this comparison between (3.40) with the original problem is to demonstrate the usefulness of the method for rapid feasible trajectory generation, (3.40) is compared with 
the solution of (3.25) instead of (3.7), as (3.25) is the close approximation to problem (3.7). As will be demonstrated, the second approach provides the most rapid trajectory generation, while the third approach is still significantly quicker than the first. It will also be demonstrated that the feasible trajectories obtained from the solution of (3.40) are reasonable compared to the optimal solutions of (3.25). The problem (3.25) is solved by the method of pseudospectral discretization [20]. In what follows, the criteria for exiting the iterations will be discussed. Furthermore, the obstacle scenarios and the comparison criteria will be presented for the problems discussed above.

For the Monte-Carlo test, 100 scenarios will be randomly designed as follows. In any of the problem scenario the initial point at $(0,0,10)$ meter and the goal point at $(100,0,10)$ meter are fixed. The $3 D$ space for the simulation test, which is also bounded by the upper and the lower bounds on the $x, y, z$ states are presented in Table 3.2. In all scenarios, the aim is to find the trajectory that avoids obstacles and remains close to the pre-defined reference path defined by the intersection of two planes $y_{a}=0$ meter and $z_{a}=10$ meter. Each scenario is initialized with state-control pairs (as given in Section 3.3.3) and obstacle location (random initialization by a uniform distribution over the $3 D$ space). The obstacle locations are defined so that they are uniformly distributed over the range $40 \sim 80$ meter along the positive $x$-axis (to avoid obstacles near the initial and the goal point), $-40 \sim 40$ meter along the $y$-axis, and $0 \sim 30$ meter along the $z$-axis. The space along the $y$ and $z$-axes were chosen so that obstacles may fall in or along the desired flight path, and thus an avoidance trajectory may be seen. In Equation (3.35), its center distances (along each axis) $a_{i}, b_{i}$, and $c_{i}$ range from $5 \sim 10$ meter, and the exponent terms $p_{x}, p_{y}$, and $p_{z}$ can range from $2 \sim 10$. Figure 3.2 shows the $3 D$ space where obstacles will be initialized. Three obstacles along the $x$ - axis can be seen as follows, a cylindrical obstacle at $x=30$ meter, a cube-shaped obstacle at $x=60$ meter, and a spherical obstacle at $x=80$ meter. The cylindrical and cube-shaped obstacles are stationary, but with different shape and size, whereas the spherical body represents a moving obstacle. The centers of stationary obstacles are fixed, whereas the position of the center of moving obstacles change. The change in position is defined as follows. Let $x_{o}, y_{o}, z_{o}$ be the position, $v_{o}$ be the velocity, and $\psi_{o}$ and $\gamma_{o}$ be the heading angle and the velocity path angle, respectively, for a moving obstacle; then, the update in the position of a moving obstacle is given by 


$$
\begin{aligned}
& \dot{x}_{o}=v_{o} \cos \left(\psi_{o}\right) \cos \left(\gamma_{o}\right) \\
& \dot{y}_{o}=v_{o} \sin \left(\psi_{o}\right) \cos \left(\gamma_{o}\right) \\
& \dot{z}_{o}=v_{o} \sin \left(\gamma_{o}\right)
\end{aligned}
$$

where $v_{o}$ is in the range $1 \sim 5 \mathrm{~m} / \mathrm{s}$ (such that it is slower than the UAV), $\psi_{o}$ in the range $-\pi \sim \pi \mathrm{rad}$, and $\gamma_{o}$ in $-\frac{\pi}{2} \sim \frac{\pi}{2} \mathrm{rad}$; furthermore, these values are randomly (uniformly) initialized in the Monte-Carlo test. Figure 3.2 shows $v_{o}$ along $\overrightarrow{O C}, \gamma_{o}(\angle B O C)$, and $\psi_{o}(\angle A O B)$ for a moving obstacle (spherical). Although a moving obstacle is shown as sphere in the figure, their shape and size are randomly (uniform distribution) initialized, as in the case of stationary obstacles.

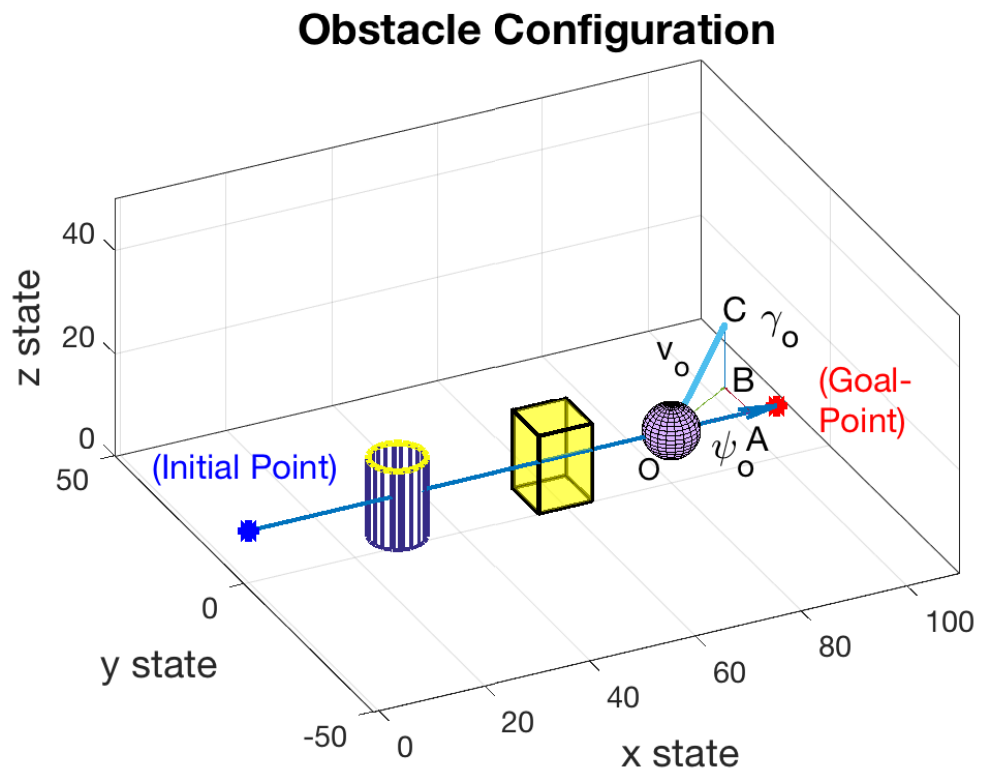

Figure 3.2: Obstacle configuration for Monte-Carlo simulation

There should be no limitation in the number of obstacles in a real application; however, the number of obstacles in the simulation is limited to 10 (the number of obstacles are randomly selected in between $4 \sim 10$ ). Among them, half of the obstacles are moving and the rest are stationary. The penalty parameters in (3.33) are now set. The penalty parameter associated with the planes $(y=0)$, which is $\beta$, and $(z=0)$, which is $\eta$, are both set to 0.1 . The parameter $\kappa$ is set to 0.7 , and the weight assigned to an obstacle $w$ is set to 0.1 . In terms of percentage, obstacle robustness has been set to $10 \%$; likewise, 
the penalty for not remaining close to the desired path has been set to $10 \%$ (for $(y=0)$ and $(z=0))$. The cost to minimize the sum of the square of distances between points has been set to $70 \%$. The value of $\epsilon_{s}$ in (3.35) has been set to 0.7 , and $\epsilon_{c}$ in Table 3.4 has been set to 0.4. Although $\epsilon_{c}$ in Table 3.4 could be a small number, the particular value was chosen for the following reasons. To change the flight path angle $(\gamma)$ in the trajectory (i.e., increase or decrease the $\gamma$ angle), bounds $\omega_{\gamma}$ and $\overline{\omega_{\gamma}}$ are required such that $\underline{\omega_{\gamma}}<0, \underline{\omega_{\gamma}}<\overline{\omega_{\gamma}}$, and $\overline{\omega_{\gamma}}>0$. From $\underline{\omega_{\gamma}}<0$, the condition for $\epsilon_{c}$ is obtained as follows:

$$
\epsilon_{c}<\frac{g \cos (\gamma)}{v}
$$

To find a maximum value of $\epsilon_{c}$ satisfying both condition (3.42) and the bounds of $\gamma$ and $v$, the right-hand side of $(3.42)$ is minimized. This yields $\epsilon_{c}<0.5781$. However, the lower and the upper bounds of $\omega_{\psi}$ in Table 3.4 depend directly on $\epsilon_{c}$. Therefore, for a reasonable rate of change of the heading angle, $\epsilon_{c}$ is set slightly lower than the allowable limit.

For all scenarios in the Monte-Carlo test, the problem is discretized using $N=16$ points, where $N$ is from (3.33). The table below presents a summary of the penalty parameters.

Table 3.5: Parameter value for the problem (3.40)

\begin{tabular}{cccc}
\hline \hline Parameters & Value & Parameter & Value \\
\hline$\kappa$ & 0.7 & $N$ & 16 \\
$\beta$ & 0.1 & $\epsilon_{s}$ & 0.7 \\
$\eta$ & 0.1 & $\epsilon_{c}$ & 0.4 \\
$w$ & 0.1 & & \\
\hline \hline
\end{tabular}

For all tests, the convergence of the sequential convex programming (SCP) algorithm to a feasible solution will be investigated starting from the initial nominal points discussed in Subsection 3.3.3. In each sequential convex programming (SCP) iteration, a convex quadratic programming (QP) problem is solved in up to 25 iterations, otherwise will be stated. As stated in Subsection 3.3.3, it many not strictly be a sequential convex programming (SCP) due to the non-convex nature of the cost function. However, the 
custom convex quadratic programming (QP) solver still works well as it is found that the minimum eigenvalue of the Hessian of the overall cost function was very small in magnitude in the case it was negative, even for the worst case scenario when initial guesses were infeasible. For example, in the case when initial guesses passed through precisely centred ten stationary obstacles along the reference path which is the worst case among Monte-Carlo, the minimum eigenvalue of the overall cost Hessian for the problem turns out to be $-1.694 \times 10^{-10}$. Thus, by contrast, the custom convex quadratic programming (QP) solver incorporates both static and dynamic regularization used in $[57,59]$ to ensure positive-definiteness of the Hessian, and prevent division by zero during the solution process, with parameters of order $10^{-7}$ in both cases. While the solvers in $[57,59]$ were developed for the case of a positive semi-definite Hessian, it is found that the algorithm works well in all cases considered in this chapter as well. The example solution is computed in a Macbook Air laptop with $1.8 \mathrm{GHz}$ processor and $4 \mathrm{~GB}$ of RAM, unless otherwise stated. For each problem, after the sequential convex programming (SCP) iteration scheme converges, the cost of the trajectory is measured using the cost function (3.4) at pseudospectral time nodes. Although the problem (3.40) has a different cost function compared to the original problem, the cost is still computed using (3.4) so that the progress in the value of cost can be compared when the original problem starts with and without the feasible starting point.

\subsubsection{Monte-Carlo Test}

Monte-Carlo test provides an estimate of the performance of the method for feasible trajectory generation under various scenarios, particularly with random obstacle definition. Thus, in this section, the cost, the number of iterations, and the computation time from (3.25) will be compared in the cases with and without a feasible starting point. The computation time required by the custom solver to obtain a feasible trajectory (problem (3.40)) will also be considered.

For all tests, the sequential convex programming (SCP) algorithm starts with $\mu=10$ and $\Delta=10$ (refer to Section 2.2 for details). The iterations are terminated once all the constraints in the problem have been satisfied (with a tolerance of $1 e-5$ ), and with the additional conditions that will be discussed below. As the decrease in the value of the cost is one of the motivating factors for proceeding further with the sequential convex 
programming (SCP) iterations, the iterations for the problem (3.25) are terminated once the absolute decrease in the value of the cost between two consecutive sequential convex programming (SCP) iteration has dropped below $\epsilon_{\tau}$. Let $C s_{i}$ and $C s_{i-1}$ be the value of the cost for the problem (3.25) at $i^{t h}$ and $(i-1)^{t h}$ iterations, respectively. Then the stopping criterion is

$$
C s_{i}-C s_{i-1} \leq \epsilon_{\tau}
$$

Furthermore, as iterations should not continue indefinitely, their number is limited to 100. In the following, for the Monte-Carlo test are summarized.

- Step 1: The problem (3.25) is solved, the algorithm stops once the stopping criterion (3.43) is satisfied. The $\operatorname{cost} C_{1}$ from (3.4), the computation time $C t_{1}$ required to solve the problem, and the number of iterations $I t_{1}$ are then measured.

- Step 2: The problem (3.40) is solved by the approach described above and the algorithm stops once all the constraints in the problem have been satisfied (with a tolerance of $1 e-5)$ and $\mu \leq 0.1$ or $\Delta \leq 0.1$ (refer to the Section 2.2 for details). Then, the states and the controls for (3.25) are extracted, as described in the last paragraph of Section 3.3.3. Finally, the method of pseudospectral discretization [20] is used to discretize the states and the controls at the pseudospectral time nodes. Then, the cost $C_{I G}$ from (3.4), the computation time $C t_{I G}$ required to solve the problem, and the number of iterations $I t_{I G}$ are measured. These states and controls now act as the starting point for the problem (3.25).

- Step 3: The problem (3.25) is solved again using the starting point from Step 2, with the same stopping criteria (3.43). Then the cost is measured as $C_{2}$ from (3.4), the computation time required to solve the problem as $C t_{2}$, and the number of iterations taken as $I t_{2}$.

In the above points, two different stopping criteria are defined for problem (3.40) and (3.25). Such stopping criteria are chosen because the aim here is only a feasible trajectory, whereas the goal of (3.25) is an optimal trajectory. Moreover, the stopping criteria for the problem (3.25) are set by equation (3.43), and $\epsilon_{\tau}=0.01$. This value is chosen because a further decrease in $\epsilon_{\tau}$ does not yield substantially better results compared to the current 
value of $\epsilon_{\tau}$; rather, it may increase the computation time and the number of sequential convex programming (SCP) iterations.

In the following, the results are discussed and compared. The table below shows the values of penalty parameters for solving the problem (3.25). Although, all the scenarios in the Monte-Carlo test converge and thus produce feasible/optimal trajectories, however it may not always be such case if obstacles are initiated near the UAV such that its flight constraint does not allow for a safe maneuver, due to its near proximity. Also, for the approach to work, the initial and the goal points should remain outside of all obstacles.

Table 3.6: Parameter value for the problem (3.7)

\begin{tabular}{cccc}
\hline \hline Parameters & Value & Parameters & Value \\
\hline$w$ & 0.5 & $\eta$ & 2.0 \\
$\beta$ & 2.0 & $\epsilon_{\tau}$ & 0.01 \\
\hline \hline
\end{tabular}

First, the problem (3.25) without a feasible starting point is compared with the problem (3.40). Figure 3.3 shows the comparison of the cost between $C_{1}$ and $C_{I G}$. The blue line shows the value of the cost using the solution of the problem (3.40), calculated at pseudospectral node points. Similarly, the value of the cost $C_{1}$ is calculated from the solution of the problem (3.25) at pseudospectral time nodes as well. The values of the cost of feasible problems are relatively higher (approximately 10 times as high) compared to $C_{1}$.

Figure 3.4 compares $C t_{1}$ and $C t_{I G}$. The results are expected owing to the stopping criteria and the nature of the problem. Similarly, Figure 3.5 shows the comparison of $I t_{1}$ and $I t_{I G}$, which is as straightforward as above. Moreover, it is interesting to note that the stopping criteria for (3.40) are satisfied after two sequential convex programming (SCP) iterations.

Subsequently, the results of solving the problem (3.25) with a feasible starting point are compared with those without a feasible starting point. Figure 3.6 shows the comparison of $C_{1}$ and $C_{2}$. As for both cases the stopping criteria are the same, the cost does not vary significantly.

Figure 3.7 shows the comparison between $C t_{1}$ and $C t_{2}$, where for almost all instances $C t_{2}<C t_{1}$. The outcome in the computation time is as expected because $C t_{2}$ has the advantage of starting with a feasible starting point. Similarly, Figure 3.8 compares $I t_{1}$ 


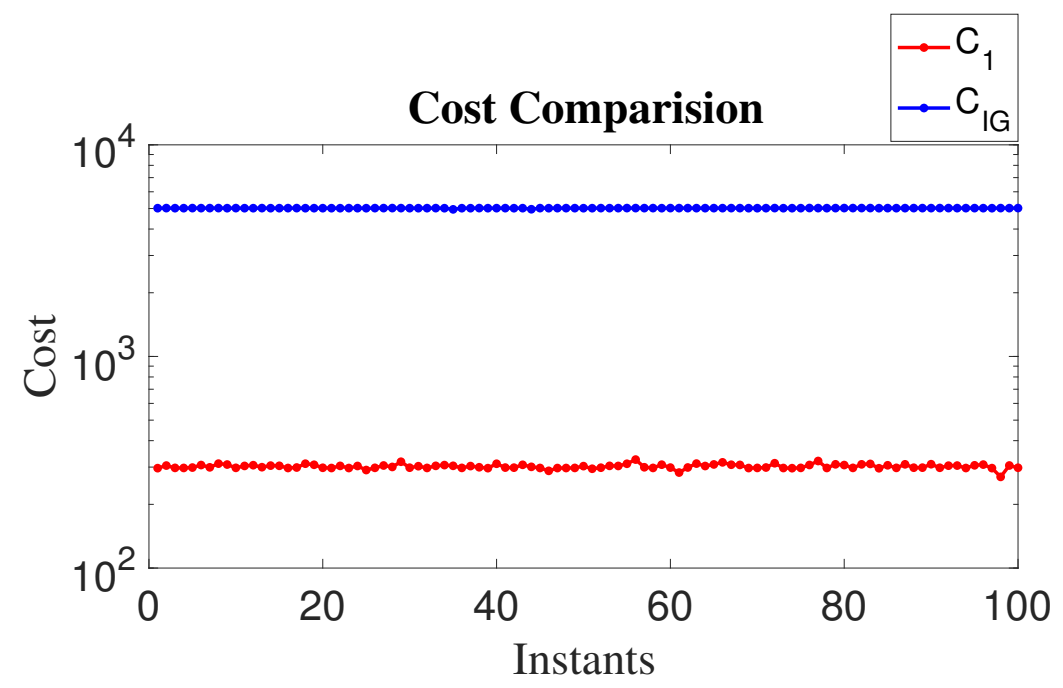

Figure 3.3: Cost comparison

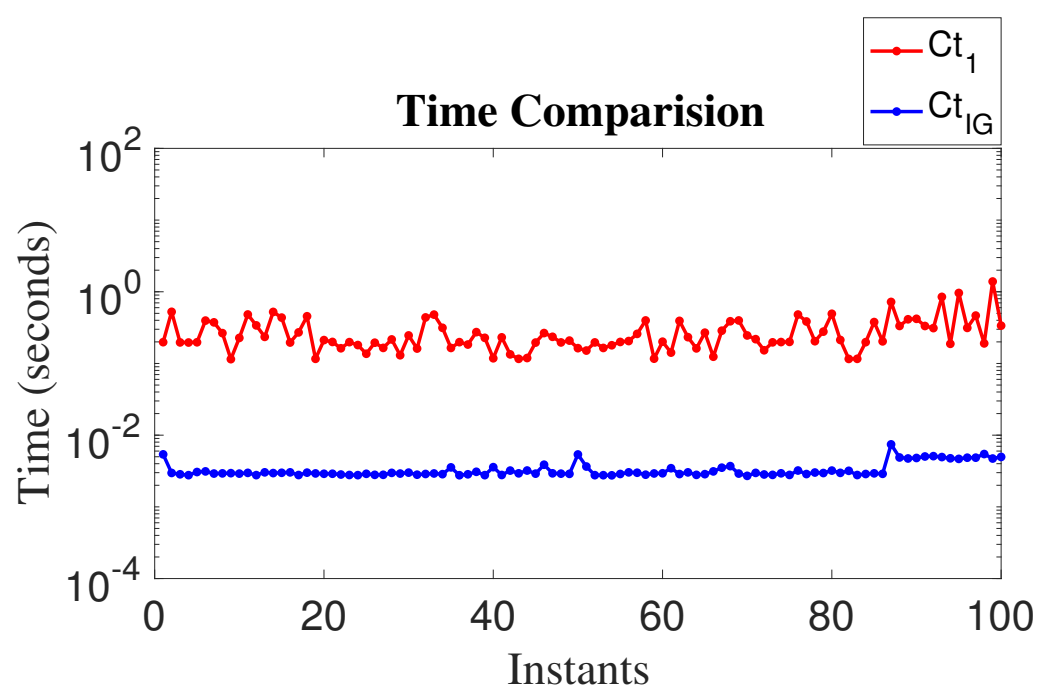

Figure 3.4: Computation time comparison 


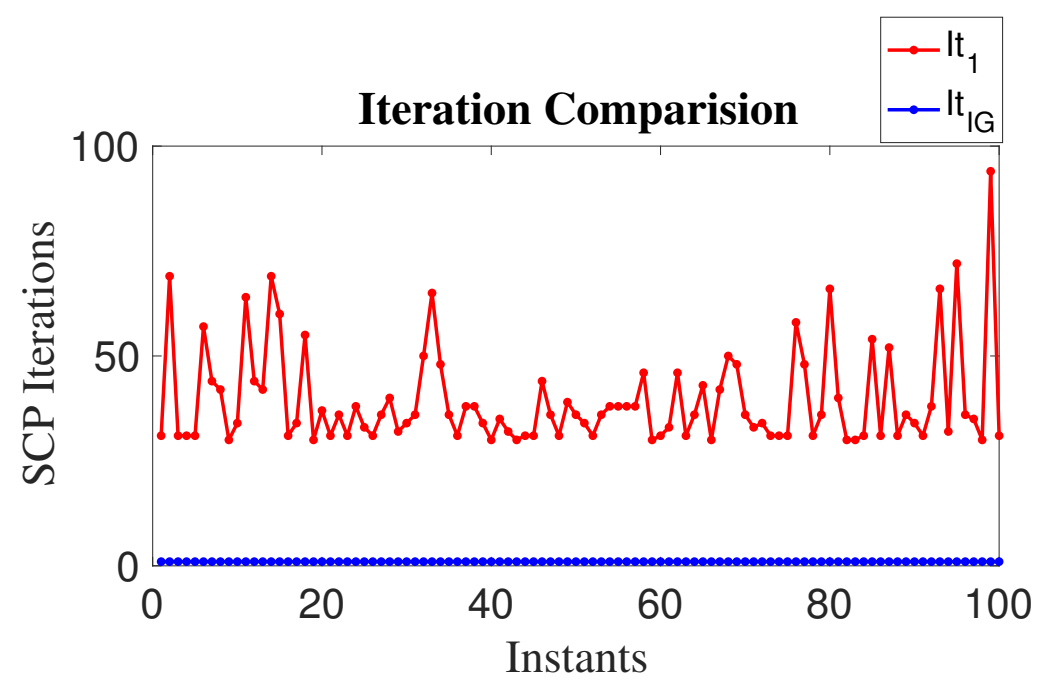

Figure 3.5: Iteration comparison

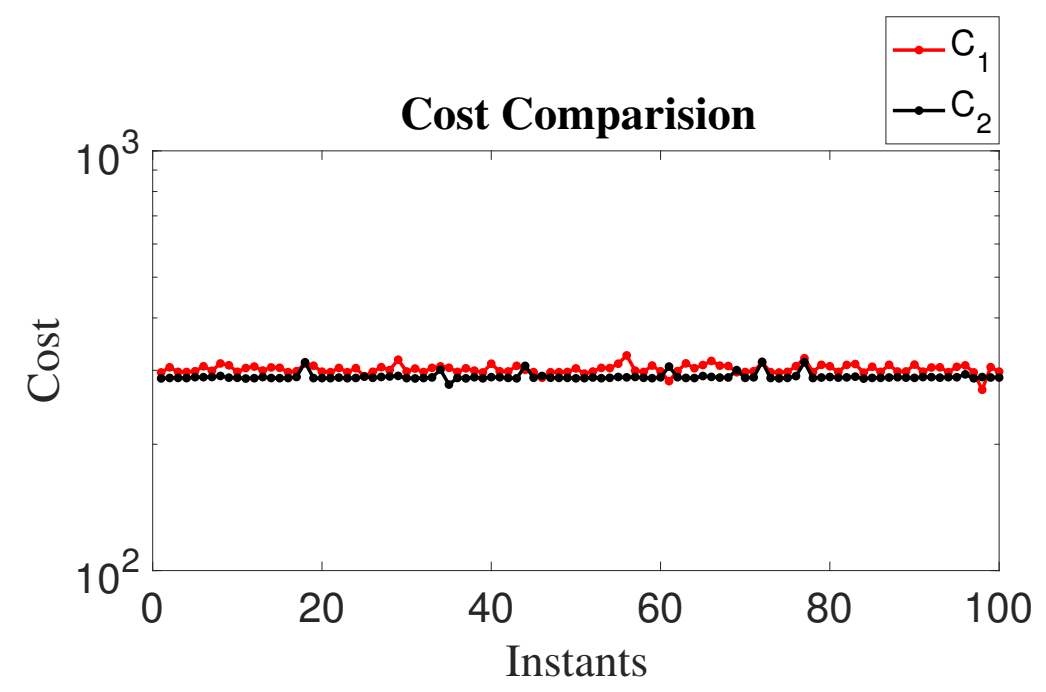

Figure 3.6: Cost comparison 
with $I t_{2}$. As the computation time is directly proportional to the number of iterations, a similar trend is observed for the number of sequential convex programming (SCP) iterations.

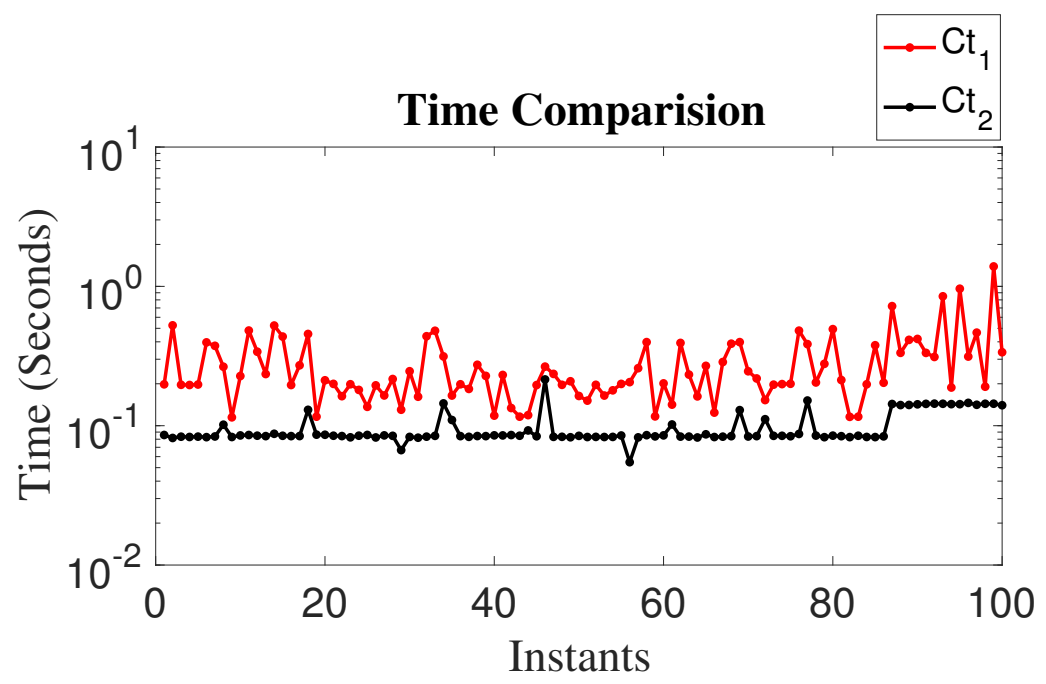

Figure 3.7: Computation time comparison

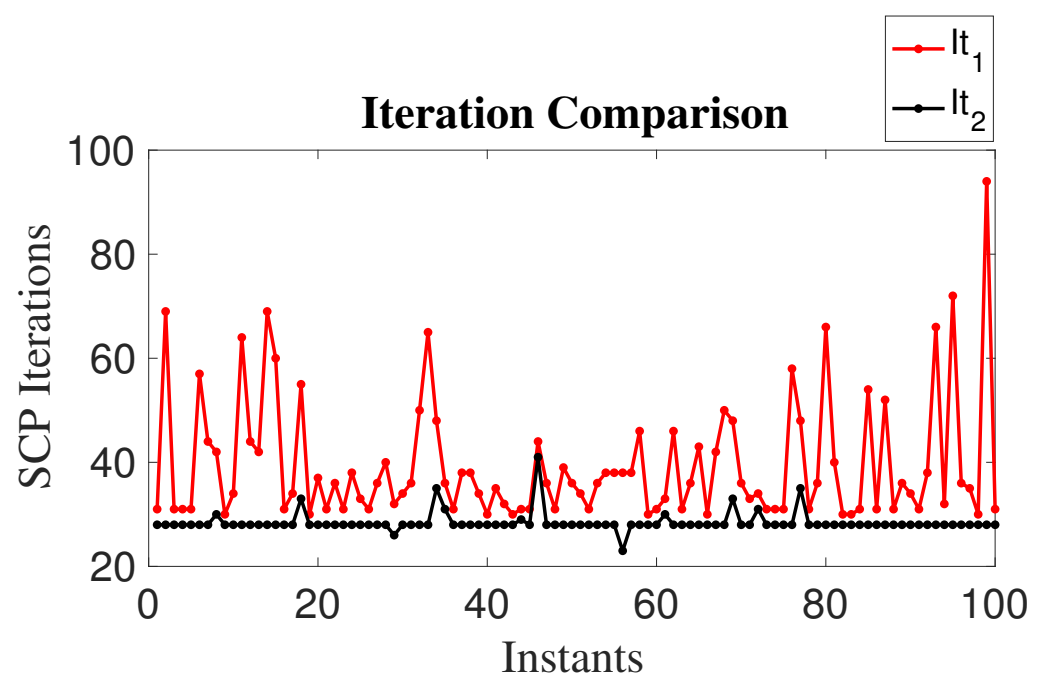

Figure 3.8: Iteration comparison

The computation time and the iteration required for solving the problem (3.25) without a feasible starting point are compared with the total computation time and the total number of iterations for solving the problem (3.40) + (3.25) with a feasible starting point, 
i.e., $C t_{1}$ versus $C t_{2}+C t_{I G}$, and $I t_{1}$ versus $I t_{2}+I t_{I G}$. Figures 3.9 and 3.10 show these comparisons. It can be seen that for all instances $C t_{1}>C t_{2}+C t_{I G}$ and $I t_{1}>I t_{2}+I t_{I G}$. Therefore, in terms of the overall improvement in the computation time, and the improvement in the number of sequential convex programming (SCP) iterations, it is beneficial to solve (3.40) with a feasible starting point and then solve (3.25) starting with the feasible trajectory.

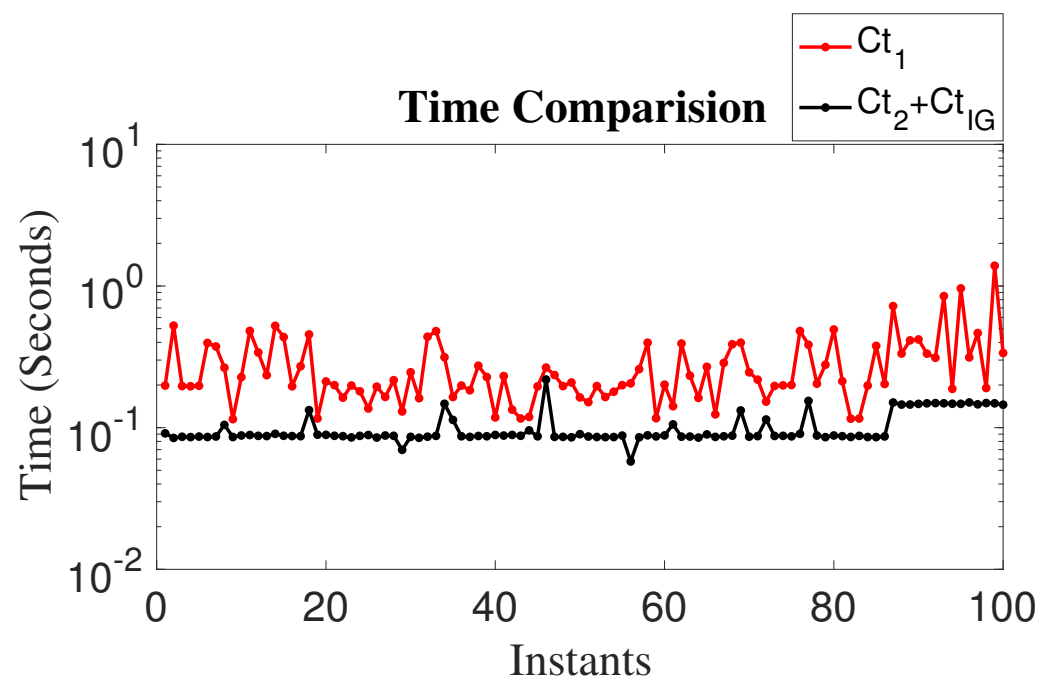

Figure 3.9: Computation time comparison between $C t_{1}$ and $C t_{2}+C t_{I G}$

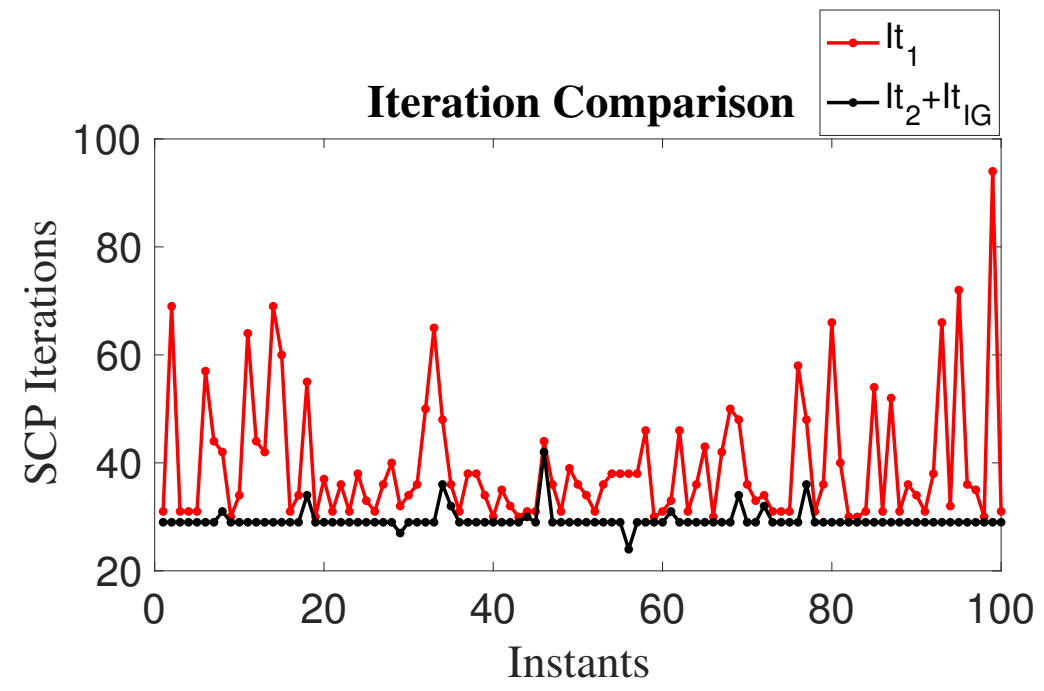

Figure 3.10: Iteration comparison between $I t_{1}$ and $I t_{2}+I t_{I G}$ 


\subsubsection{Comparison Between Original and Simplified Problem}

As stated in Section 3.2.4, there is no guarantee that the solution to the simplified problem (3.25) is locally optimal for the original problem (3.7), but instead we get a suboptimal feasible solution. Thus to demonstrate that, in Figure 3.11 Monte-Carlo comparison between the problems (3.25), simplified problem and (3.7), original problem are presented in terms of the value of the cost. The comparison shows that the solution to (3.25) yields cost very similar to the solution to (3.7) when both of the problems start with the same feasible starting point.

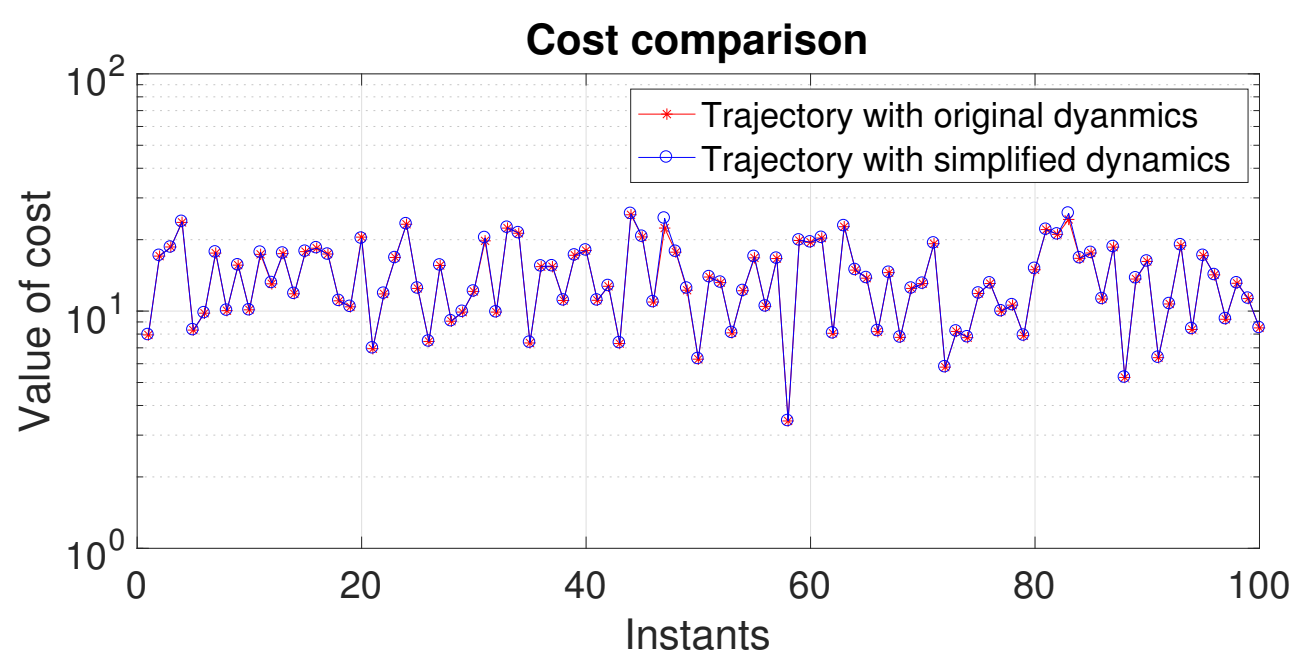

Figure 3.11: Cost comparison between the original and the simplified problem with Monte-Carlo test.

\subsubsection{Comparison with DIDO, ECOS and IPOPT}

In order to present the gain that could be made with the custom solver (referred to as CS), the Monte-Carlo test is performed using DIDO, ECOS, IPOPT and CS under the same conditions, and are compared in terms of the value of cost, and the computation time. For the Monte-Carlo simulations, one hundred test scenarios are performed with 16 node using a Macbook Pro with 2.9 Ghz processor and 8 GB RAM. The scenarios for the Monte-Carlo test are initialized as in Section 3.4.2.

Although there are various NLP solvers to solve the problem of our type we choose IPOPT with ma57 as linear solver, as this solver is also used for trajectory optimization 
[94]. DIDO is chosen, since it uses the SNOPT as its solver to solve optimal control problem with Pseudospectral discretization [20], and its been often used for robotics applications. Finally, ECOS which solves second order cone problem is chosen, as it can be adjusted to solve QP problems (similar to those in this thesis) and the solver is designed for embedded application. The comparison between the solvers uses the same steps of solving as in Section 3.4.2. First, the simplified problem is solved without a feasible initial guess. Then, the problem for feasible trajectory is solved, where the solution is considered as a feasible initial guess. Finally, the simplified problem is solved with the feasible initial guess. Apart from DIDO which uses SNOPT internally, we use ECOS, IPOPT and CS within the SCP framework for a fair comparison where $M E X$ (in Matlab environment) versions of the solvers were used.

In Figure 3.12 the value of the cost obtained after solving the simplified problem with feasible initial guess are compared between the use of solvers; DIDO, IPOPT, ECOS and CS. In the comparison, the value of the cost for all the solvers are either exactly the same (within second decimal place) or are very close.

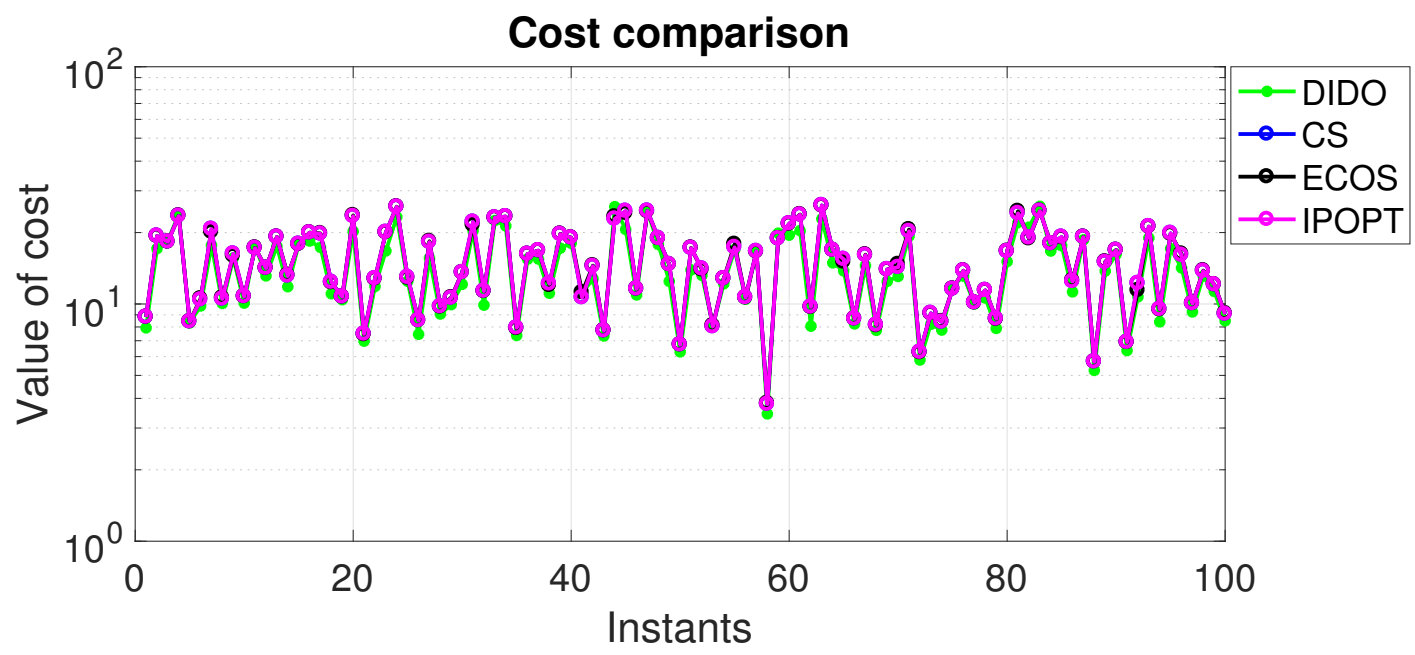

Figure 3.12: Cost comparison between DIDO, IPOPT, ECOS and CS to solve simplified problem with feasible initial guess.

In Figure 3.13 the computation time used by the solvers to solve the simplified problem with feasible initial guess are compared. In all the cases the computation time with CS is the lowest while it is highest with DIDO. Since DIDO uses the SNOPT (a general solver) as its solver to solve the discrete (Pseudospectral) optimization problem it is slower as 
expected. IPOPT, while it is also a general solver, a $M E X$ version of the solver was used within the SCP framework. ECOS, designed to perform in embedded system was used in $M E X$ form to speed up the computation. Finally, the custom solver designed to operate with only non-zero terms was used in $M E X$ form, which significantly lowered the solution time.

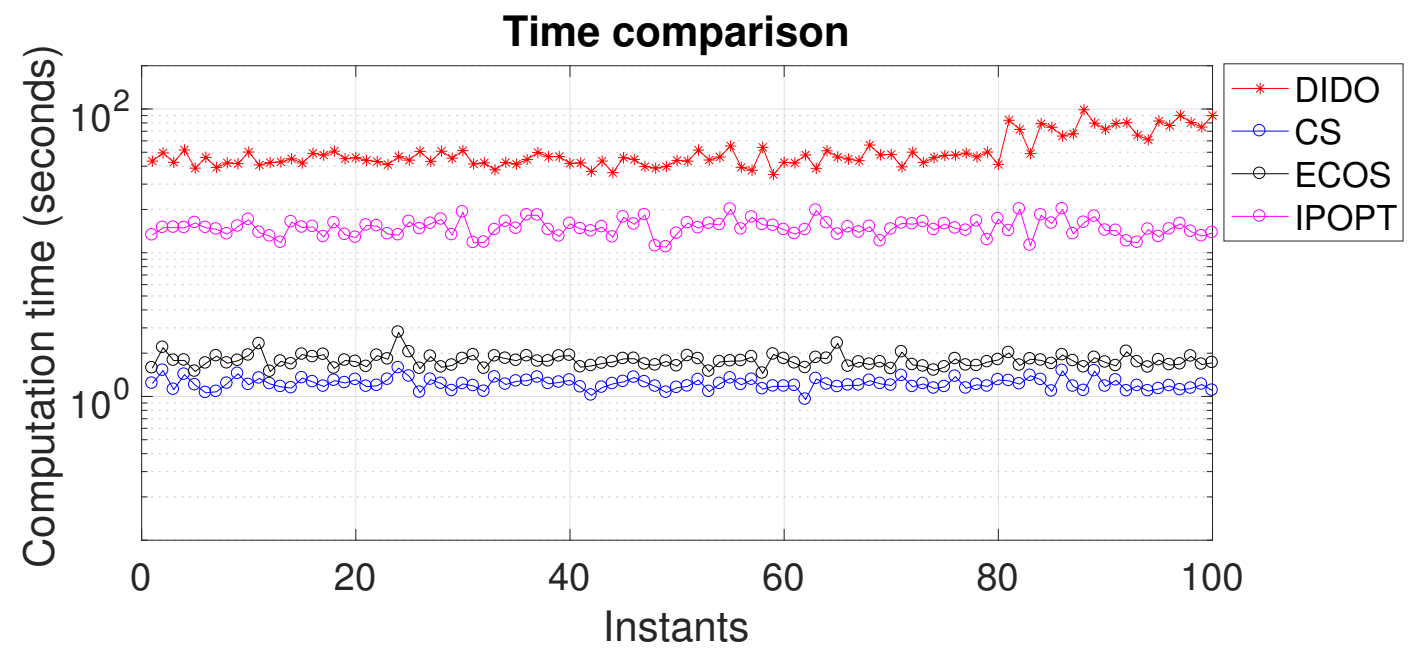

Figure 3.13: Computation time comparison between DIDO, IPOPT, ECOS and CS to solve simplified problem with feasible initial guess.

Table 3.7 presents the maximum and the average computation time comparison. As expected, the CS has the lowest computation time while the DIDO has the highest. In terms of computation time CS and ECOS are close, while CS is more than five time faster than IPOPT and more than forty time faster than DIDO.

Table 3.7: Comparison of computation time between the solvers

\begin{tabular}{ccc}
\hline \hline & Average computation time $(\mathrm{s})$ & Max computation time $(\mathrm{s})$ \\
\hline $\mathrm{CS}$ & 1.22 & 1.58 \\
\hline ECOS & 1.77 & 2.79 \\
\hline IPOPT & 14.95 & 20.10 \\
\hline DIDO & 51.02 & 98.74 \\
\hline \hline
\end{tabular}




\subsubsection{Example: A Stationary and a Moving Obstacle}

An example with two obstacles is now considered. One is a spherical stationary obstacle at $(40,1,10)$ meter with a radius of 8 meter. The other is a moving spherical obstacle with a radius of 8 meter. The moving obstacle starts at time $t=0$ second from $(60,40,10)$ and move along the $x$-axis at a velocity of $4.5 \mathrm{~m} / \mathrm{s}$ with the heading angle of $-\frac{\pi}{2}$ radian and a flight path angle of 0 radian. Figure 3.14 shows the obstacle configuration for this example. A UAV should start from the initial point at $(0,0,10)$ meter with velocity of $10 \mathrm{~m} / \mathrm{s}$, flight path angle and heading angle of 0 radians, and should reach the target point at $(100,0,10)$ meter, while avoiding the stationary and the moving obstacles.

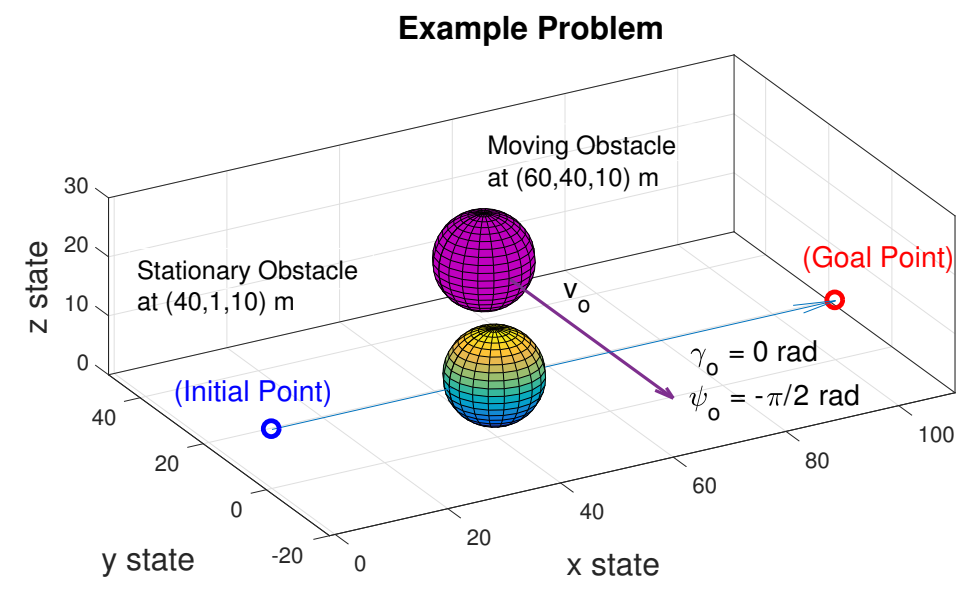

Figure 3.14: Obstacle configuration for the example

This example problem of feasible trajectory generation is solved using the model problem (3.40) with $N=25$ node points. The related parameters are presented in Table 3.1 (and Table 3.5 where applicable). sequential convex programming (SCP) is applied, and the iterations terminate once all the constraints have been satisfied (with a tolerance of $1 e-5)$ and $\mu \leq 0.1$ or $\Delta \leq 0.1$ (refer to the Section 2.2 for details). Three iterations are required to satisfy the stopping criteria. A further discussion on the stopping criteria by the use of infeasible start newton method is present in Section 3.4.6. The output is the position of the points $(X, Y, Z)$ and the corresponding velocity $V$. Initially, time is 
obtained using Equation (3.27), as follows:

$$
\begin{gathered}
t_{1}=0 \\
t_{i}=t_{i-1}+\delta t_{i-1}
\end{gathered}
$$

where $i=2, \ldots N-1$, and $\delta t_{i-1}$ is given by Equation (3.27).

Then, the acceleration $a$, the rate of change of the flight path angle $\omega_{\gamma}$, and the rate of change of the heading angle $\omega_{\psi}$, which are the controls for the kinematic model (3.23), are obtained using Equations (3.30), (3.31), and (3.32), respectively. Figures 3.15-3.17 show the plot for these kinematic controls with their respective bounds, where the expression for bounds are summarized in Table 3.4.

Figure 3.15 shows the acceleration, which lies within the upper and the lower bounds, as shown in the figure. The lower and the upper bounds on the acceleration are computed from (3.17) and (3.18) respectively. Figure 3.16 shows $\omega_{\gamma}$ between its upper and the lower bounds, which are computed from (3.19) and (3.20), respectively. Similarly, Figure 3.17 shows $\omega_{\psi}$, lying within its upper and the lower limits, which are computed from (3.21) and (3.22), respectively.

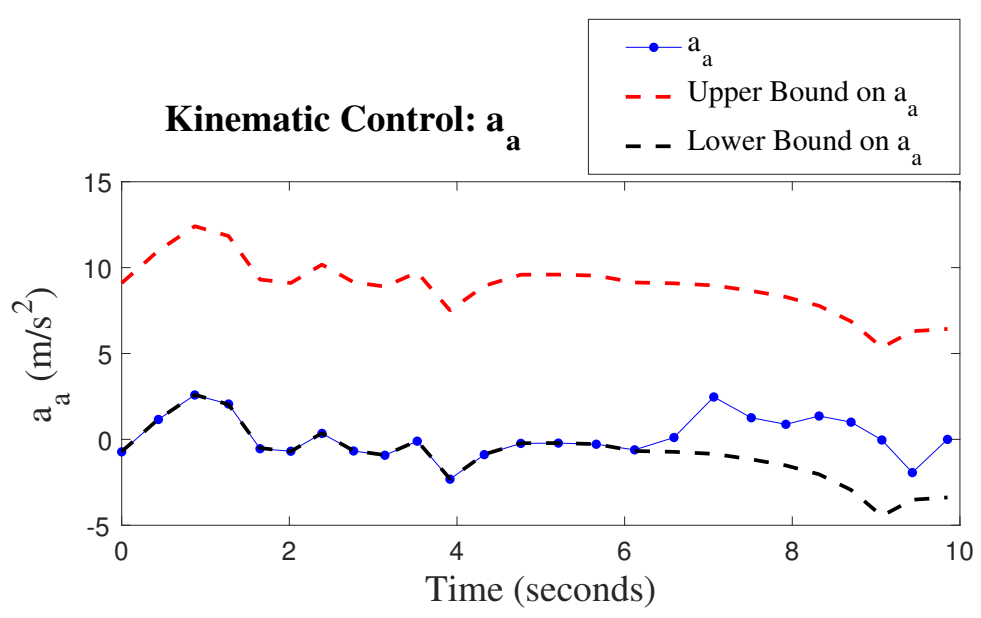

Figure 3.15: Acceleration $a$

Once the kinematic controls have been computed, Equations (3.14)-(3.16) are used to compute the controls for the dynamic model (3.1): thrust, $\phi$, and $\alpha$. However, the values from (3.14)-(3.16) are taken as a starting point, and Equations (3.8)-(3.10) are iteratively solved before the approximations to obtain the final values of the controls. 


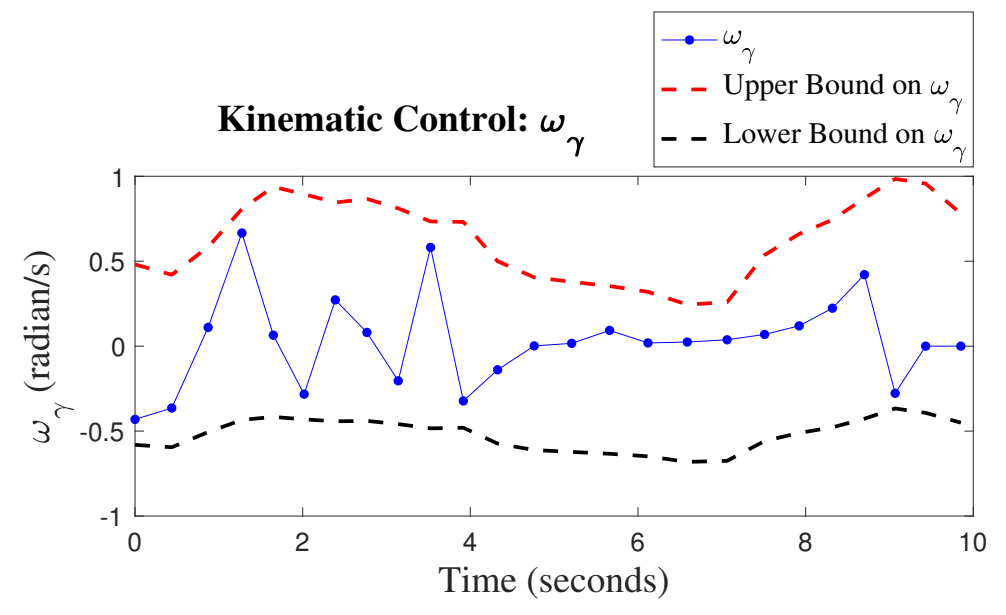

Figure 3.16: Rate of change of flight path angle $\omega_{\gamma}$

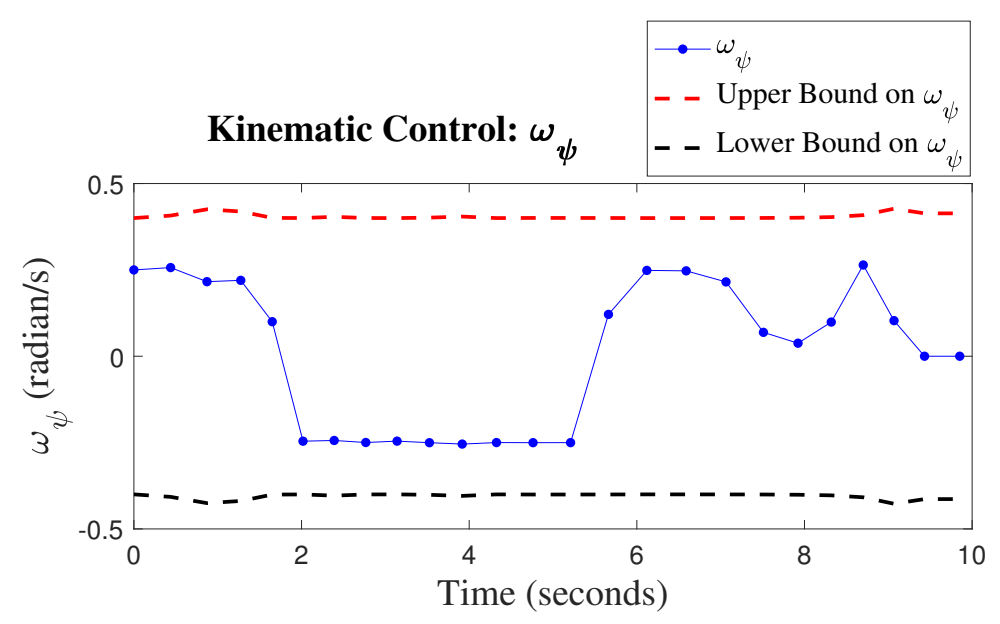

Figure 3.17: Rate of change of heading angle $\omega_{\psi}$

Figures 3.18, 3.19, and 3.20 show the thrust, the roll angle $\phi$, and the angle of attack $\alpha$, respectively, where all the controls for the dynamics model (3.1) lie within their upper and the lower bounds (if they do not lie within their bounds then the controls are infeasible and the UAV cannot achieve the trajectory). The limits for the controls of the dynamic model are given in Table 3.3. Figures 3.18-3.20 verify that solving the feasibility problem (3.40) can still generate feasible controls for the dynamic model (3.1).

The results of the integration of dynamic controls into the dynamic model (3.1) are now presented. Figures 3.21-3.23 show the arrangement of points (blue line with dots), which are the solution from the solver, and the red colored line, which is the output of 


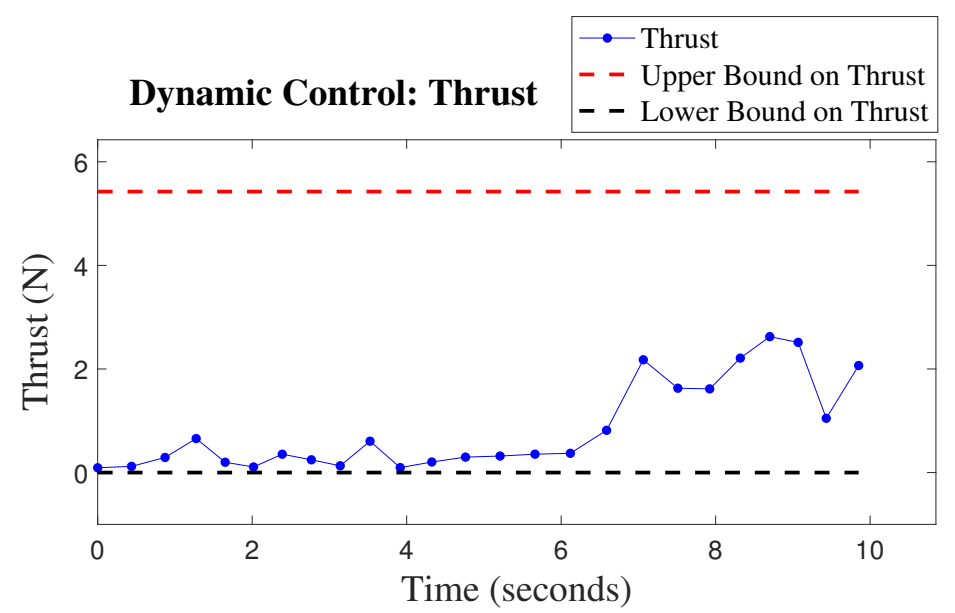

Figure 3.18: Thrust $T$

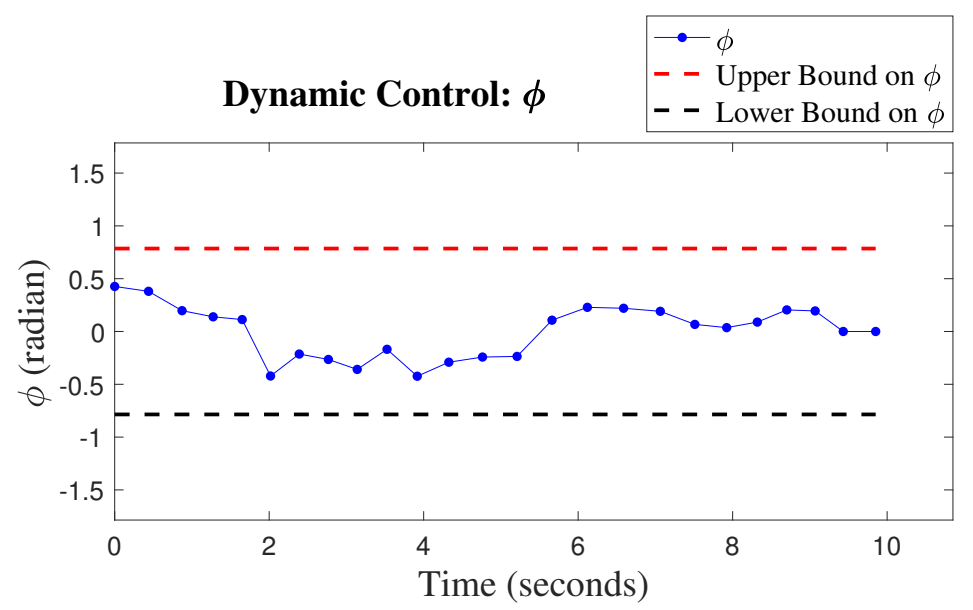

Figure 3.19: Roll angle $\phi$ 


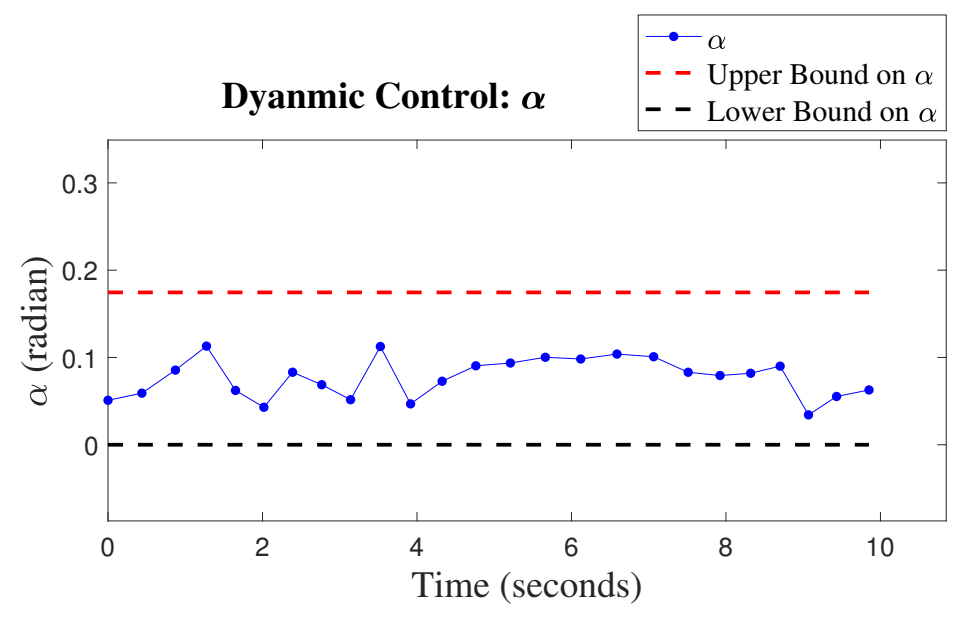

Figure 3.20: Angle of attack $\alpha$

the Runge-Kutta integration of the dynamic controls into (3.1).

Figure 3.21 shows a $3 D$ view of the example scenario, including the initial and the goal point. The black line joining the black dot represents the trajectory of the moving obstacle. To understand the location of an obstacle and the UAV in the scenario, the green and magenta lines are drawn. They show the location of the UAV and an obstacle at a time instant. The green colored lines are used to indicate that the UAV has not yet reached the line $y=60$ meters, whereas the magenta lines indicate that the UAV has crossed the line. The moving obstacle starting from $(60,40,10)$ moves towards the postive $x$-axis to intercept the UAV, which, however, passes underneath the moving obstacle to avoid it, and takes slightly longer time to reach the intersection point.

Figures 3.22 and 3.23 show the $\mathrm{XZ}$ and $\mathrm{XY}$ views of Figure 3.21. In Figure 3.22, it is seen that that the UAV avoids the obstacle from underneath, whereas Figure 3.23 shows that the UAV deviates more from the $x$-axis. In addition, the red line in Figures 3.213.23 shows the feasibility of the dynamic controls. Feasibility implies that the dynamic controls, when integrated into Equation (3.1), provide approximately the same position as the solution from the solver. It can be observed that the trajectory from the custom solver deviates more from the desired reference planes $y=0$ and $z=10$ owing to the choice of the penalty parameters given in Table 3.5, and to the avoidance condition for the moving obstacle. However, it is seen in Figure 3.23 that the path tends to bend toward the plane $y=0$ after $x=70$ meters, after the avoidance of the moving obstacle. 


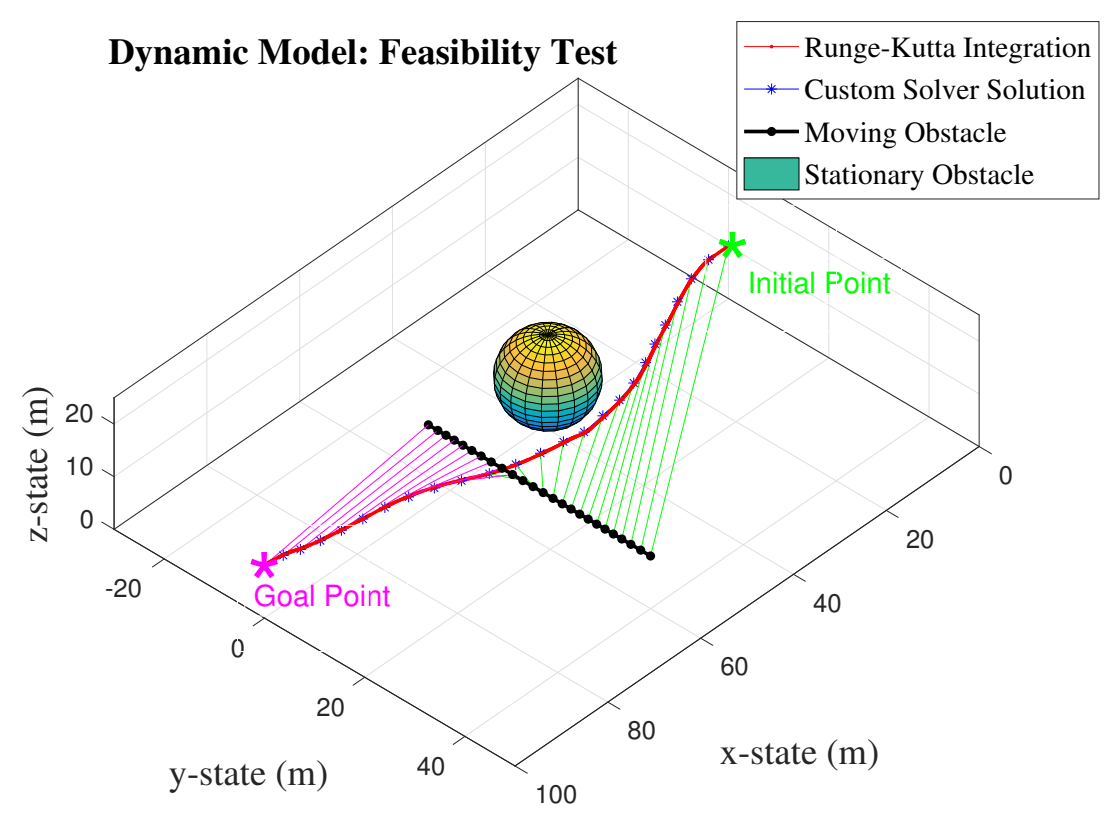

Figure 3.21: Feasibility test of dynamic controls

Similarly, in Figure 3.22, the UAV tends to bend toward $z=10$ after $x=70$ meters, which is not notable until $x=90$ meters.
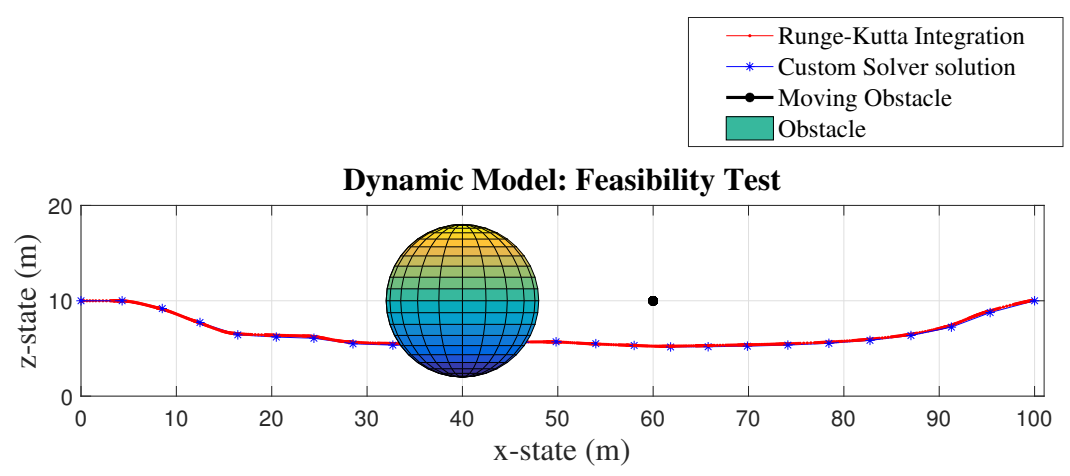

Figure 3.22: $\mathrm{XZ}$ view of the obstacle avoidance scenario

Figures $3.24,3.25$, and 3.26 show the velocity, the heading angle, and the flight path angle, respectively, in the red-colored line, which is the outcome of the dynamic controls integration, and the blue colored line with a dot representing the solver solution. It can be verified that velocity, heading angle, and flight path angle lie within their upper and the lower bounds, which are shown in Table 3.2. 


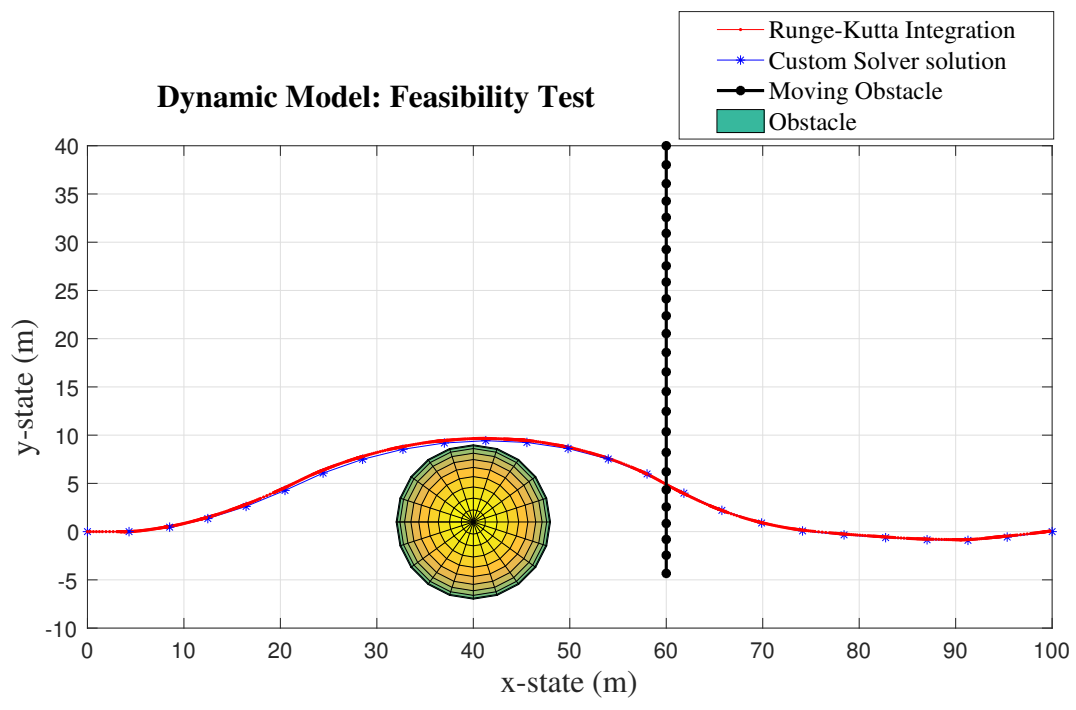

Figure 3.23: XY view of the obstacle avoidance scenario
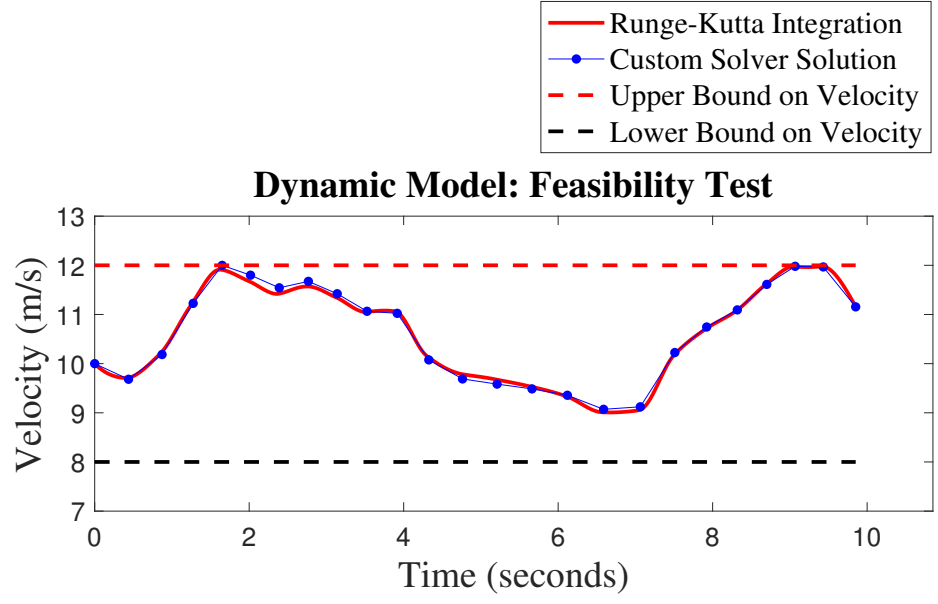

Figure 3.24: Velocity $v$ 


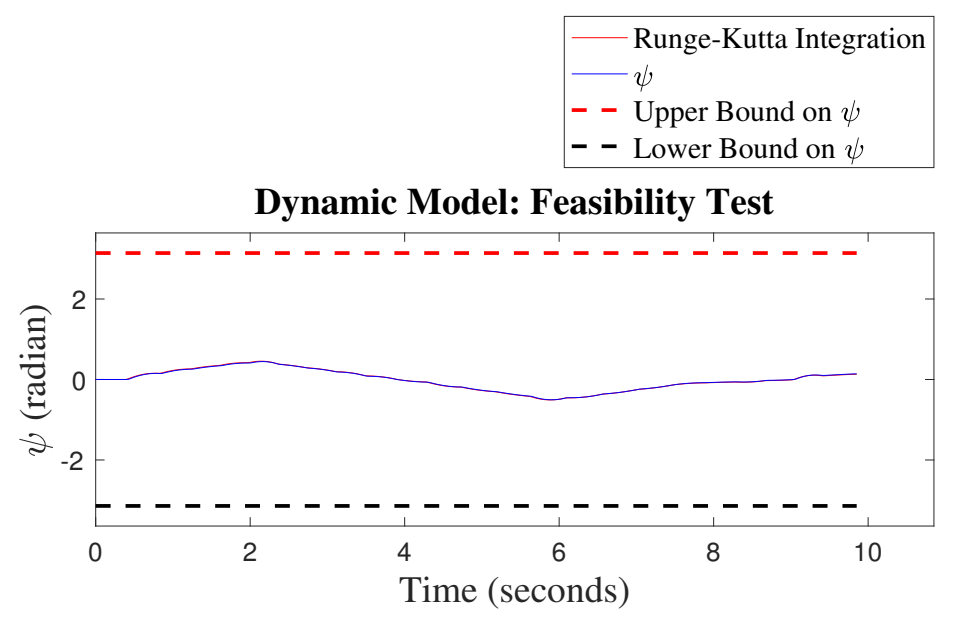

Figure 3.25: Heading angle $\psi$

Figure 3.27 shows that the UAV successfully avoids the moving obstacle at the node points. The red line represents the radius of the moving obstacle, and the blue line represents the distance between the UAV and the moving obstacle. The UAV reaches as close as 9.9 meters to the moving obstacle at 5.7 and 6.1 seconds during the flight.
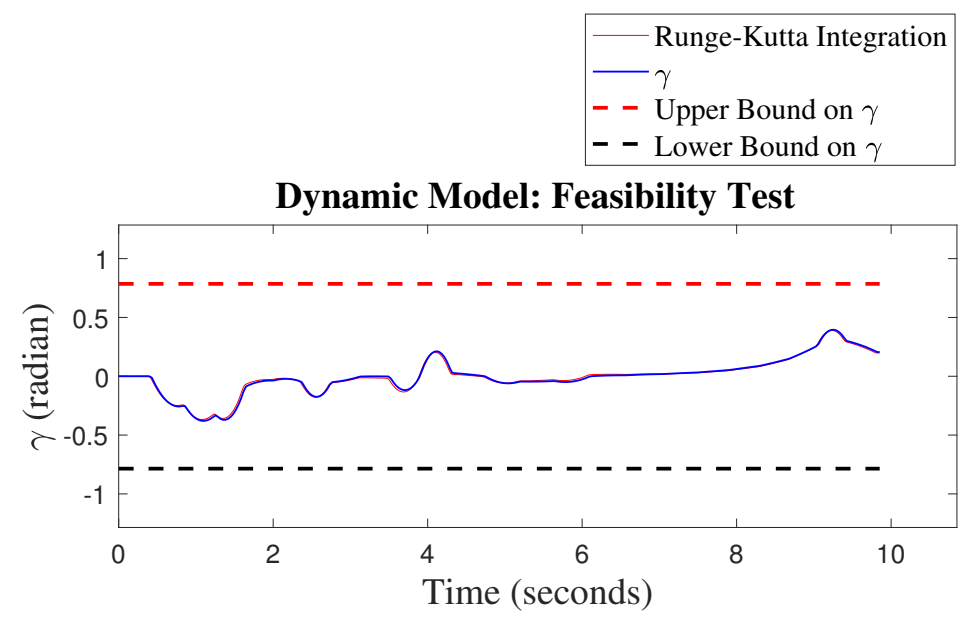

Figure 3.26: Flight path angle $\gamma$

Finally, Figure 3.28 shows the value of the cost function from (3.40) at the end of each sequential convex programming (SCP) iteration. There are two types of lines: a bluecolored line that overlaps with the red-colored lines but stops after the third iteration, and a red-colored line that does not stop until iteration 15 . The red colored line shows how 


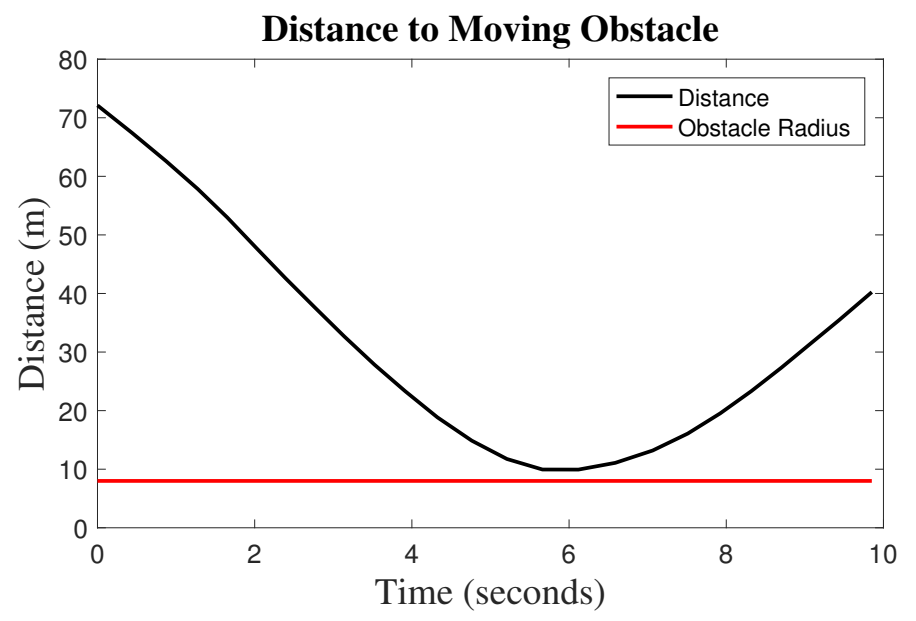

Figure 3.27: Distance to moving obstacle

the sequential convex programming (SCP) method reduces the cost over the iterations.

Figure 3.29 shows the computation time in milliseconds for solving the problem (3.40) on a laptop (Intel $\mathrm{CPU}$ at $1.8 \mathrm{GHz}$ and $4 \mathrm{~GB}$ of RAM). The computation time shown is the time required by the custom solver during each sequential convex programming (SCP) iteration. As the sequential convex programming (SCP) method stops after the third iteration (shown by the blue-colored line), 27.25 milliseconds are required for a feasible trajectory. The red-colored line shows the computation time for all 15 sequential convex programming (SCP) iterations.

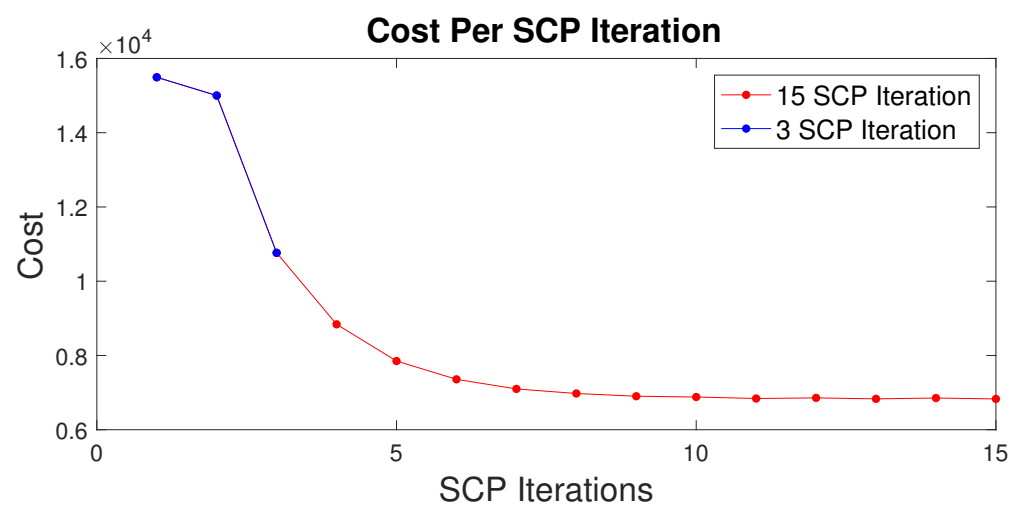

Figure 3.28: The progress in the cost for 15 SCP iterations

Further, the computation time is presented for a credit-card sized computer with an ARM (Advanced RISC Machines, RISC: reduced instruction set computer)-based proces- 


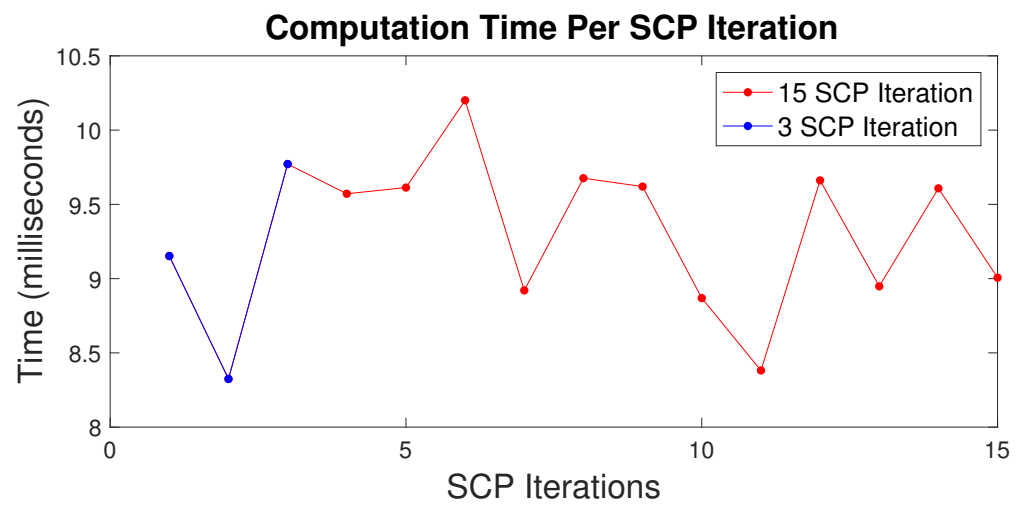

Figure 3.29: The computation time for 15 SCP iterations

sor (which has the potential of being an onboard computer for UAVs). The computation time (in milliseconds) shown in Figure 3.30 is for the above example scenario in a Raspberry PI 3B computer [95] (1.2GHz Broadcom CPU and 1GB RAM). The ability of such computers for feasible trajectory generation is demonstrated. However, there exist other ARM (Advanced RISC Machines)-based computers with similar physical size but higher computing power. Therefore, based on Figure 3.29, where a feasible trajectory is generated after three sequential convex programming (SCP) iterations, approximately 667.8 milliseconds (solution time for three iterations are added) are required for the Raspberry PI 3B computer to obtain a solution.

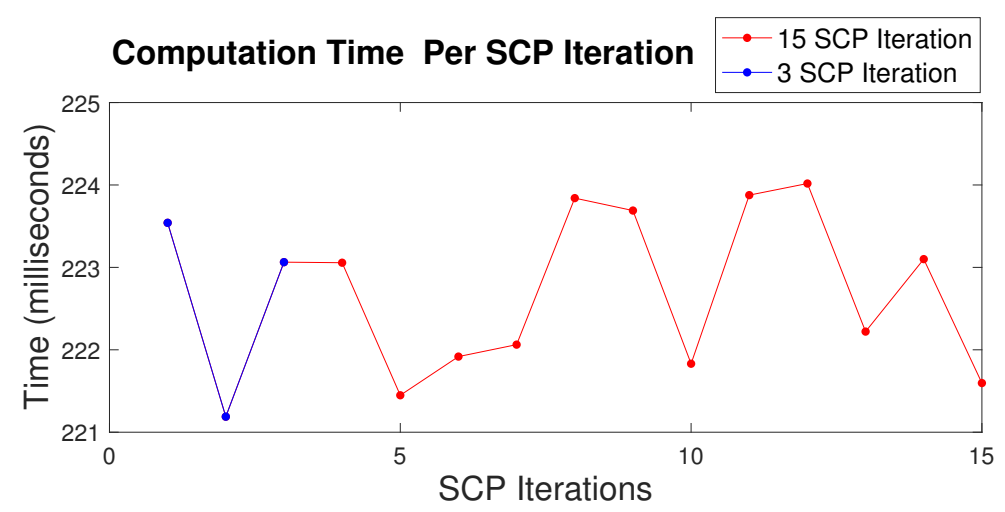

Figure 3.30: Solution time for Raspberry PI 3B (15 SCP iterations) 


\subsubsection{Example: Stationary Obstacles at the Centre of a Refer- ence Path}

In this subsection, some of the challenging scenarios are considered in which obstacles are centered exactly at the center of a reference path that a UAV is supposed to take. The numerical examples are undertaken to demonstrate that, even if initial guesses are within or at the boundary of obstacles (initialization is given inside obstacles boundary), the infeasible start method in 2.2.1 solves for a feasible trajectory. As similar to the example in Section 3.4.5, a UAVs initial point should be $(0,0,10)$ meters but with velocity, flight path angle and heading angles as $8 \mathrm{~m} / \mathrm{s}$, and 0 radians respectively. Likewise, the target point should be at $(100,0,10)$ meter. While the aim of the UAV is to remain close to a reference line, there are three spherical obstacles centered exactly at the reference line at the center distance of 20 meters apart starting from $x=40$ to $x=80$ meters, with their spherical radii all being 10 meters. Obstacles are distributed starting 40 meters away from the initial point, because of the initial conditions the UAV needs maneuvering space to avoid the obstacles. This scenario is solved using the problem (3.40) with $N=16$ node points, where problem parameters are as shown in Table 3.1 (and Table 3.5 where applicable), with a starting value of $\zeta=2$ as in (2.11). As in Subsection 3.4.5, the sequential convex programming ( $\mathrm{SCP}$ ) method is used to solve the problem with exactly the same terminating parameters. As the approach to obtain controls (the kinematic controls and subsequently the dynamic controls) from the optimization parameters like $(X, Y, Z, V)$ has been shown in the earlier example including solution time and the number of iterations required, in this example, such details are not sought. Rather, feasible trajectories starting from different initial guesses are presented such that the Infeasible Start Newton Method as stated in 2.2.1 is required. This study will show the usefulness of Infeasible Start Newton Method as in 2.2.1, for feasible trajectory generation where a resulting quadratic programming $(\mathrm{QP})$ problem is non-convex. In order to study that, four sub-cases for problem (3.40) based on initial guesses provided are formed as follows,

\section{- Initial Guess 1:}

In the first case, the initial guess to problem (3.40) is located very close to obstacles with few points at the boundaries of the obstacles, as shown in Figure 3.31 with the following values, 
$x$-axis : Equispaced points from origin to the goal point.

$y$-axis : Points at $y=10$, besides the origin and the goal point with $y=0$.

$z$-axis : Points at $z=10$.

Figure 3.32 shows the initial guesses with their respective legends. From this example case, a feasible trajectory is obtained to compare it with other cases.

\section{- Initial Guess 2:}

In the second case, the initial guess to problem (3.40) is again provided very close to obstacles with some points at the boundaries of the obstacles, but this time the points are above obstacles, as shown in Figure 3.31 as follows,

$x$-axis : Equispaced points from origin to the goal point.

$y-$ axis : Points at $y=0$.

$z$-axis : Points at $z=20$, besides the origin and the goal point with $z=10$.

Figures 3.33, and 3.34 clearly show the initial guesses with their respective legends. In this case, the initial guess points are arranged such that a fixed-wing UAV has to take a large flight path angle to fly over obstacles. However, such flight trajectory may not be possible due to the given UAV parameters. Thus, the output trajectory from this example will show a truly feasible trajectory for the UAV despite the given initial guess.

- Initial Guess 3:

The third case is the mirror case to the first case where the initial guess to the model problem is provided as follows,

$x$-axis : Equispaced points from origin to the goal point.

$y-$ axis : Points at $y=-10$, besides the origin and the goal point with $y=0$.

$z$-axis: Points at $z=10$.

Again, in this case, some points in the initial guess are at obstacles' boundaries. Figure 3.32 shows the arrangement of points for this case. The aim of this example is to compare the output trajectory with the first case.

- Initial Guess 4:

Finally, for the fourth case, the initial guess to the problem is provided such that the points lie inside or exactly at the center of obstacles as follows, 
$x$-axis : Equispaced points from origin to the goal point.

$y-$ axis : Points at $y=0$.

$z$-axis : Points at $z=10$.

This is one of the challenging cases where some points in the initial guess and obstacles' centers are the same. Figures 3.31, 3.32, and 3.33 show the points distribution where legend indicates Initial Guess 4. For this case, since all the points in the initial guess lie exactly on the reference path with some points at the center of obstacles, a resulting feasible trajectory may choose any side outside of obstacles. However, numerically the problem is not solvable when a point in an initial guess and an obstacle center are exactly the same. This is mainly due to the obstacle constraint which becomes unbounded below when the natural logarithm goes to zero (refer to equation (3.45)). Therefore, to solve the problem a number $\epsilon_{I D}=(2 * \operatorname{rand}(1,1)-1) * 10^{-7}$ is added (similar to the order of static regularization (2.20) performed in Chapter 2, which is $10^{-7}$ ) to an obstacle constraint as follows,

$$
\begin{aligned}
h_{j}(x, y, z)= & \ln \left[\left|\left(\frac{x-x_{c}+\epsilon_{I D}}{a_{j}}\right)\right|^{p_{x_{j}}}+\left|\left(\frac{y-y_{c}+\epsilon_{I D}}{b_{j}}\right)\right|^{p_{y_{j}}}+\right. \\
& \left.\left|\left(\frac{z-z_{c}+\epsilon_{I D}}{c_{j}}\right)\right|^{p_{z_{j}}}\right] \geq \epsilon_{s}>0
\end{aligned}
$$

where all the parameters related to the expression have been defined in (3.3). Although it may make more practical sense to perturb the initial points given to the example problems rather than to perturb obstacle location (which are the actual locations of obstacles), these perturbation in the obstacles' center are very small. Also, the same obstacle constraint is used for all the four example scenarios in this subsection.

Figure 3.31 shows the distribution of points for the four cases of initial guesses. The initial and goal point has been indicated in the figure. In among the initial guesses, the first guess is distributed at the left side of obstacles, while the second is above the obstacles. The third guess is at the right side of obstacles, while the fourth passes through the obstacles.

Figure 3.32 shows the XY view of the arrangement of the obstacles. For a UAV that goes from the initial to the goal point, the Initial Guess 1 and the Initial Guess 3 are 


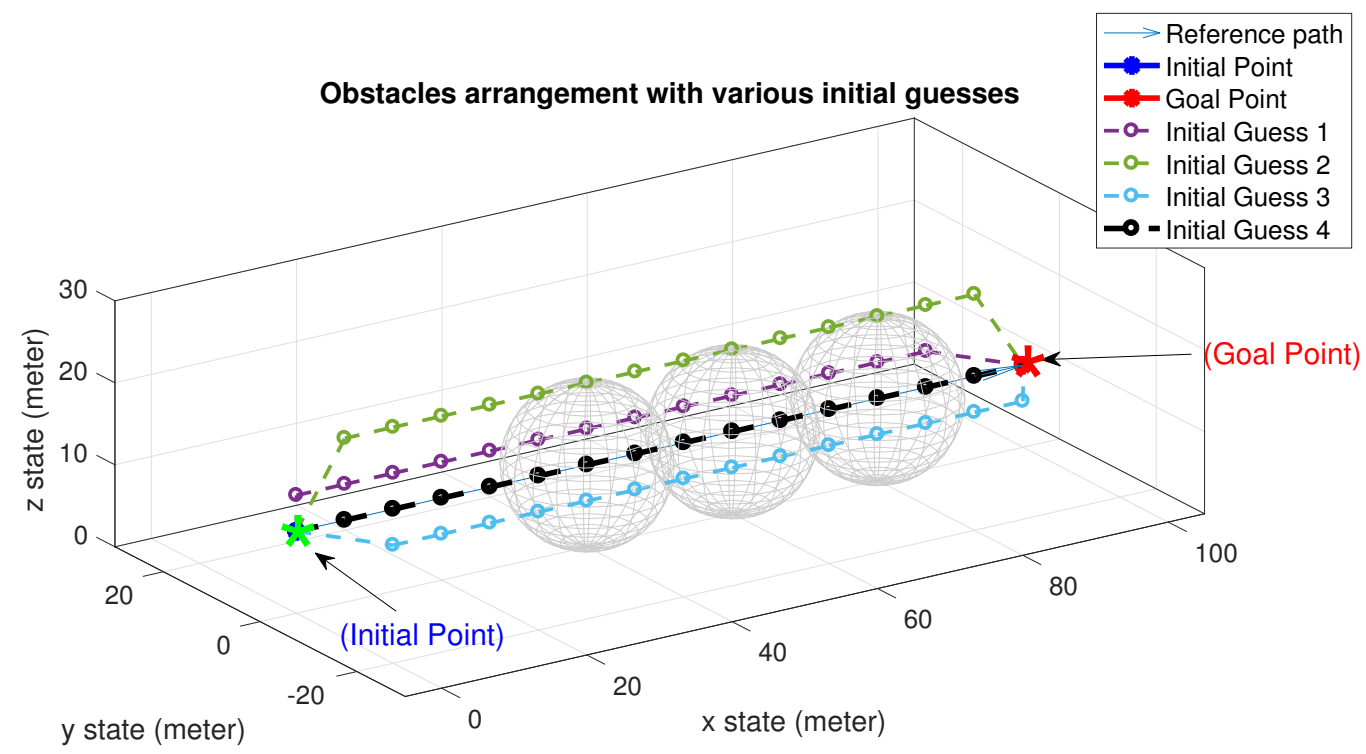

Figure 3.31: Stationary obstacles with various initial guesses, 3D view

distributed on either side of obstacles, where the points are at the distance of 10 meters away from the reference path and some points are at the boundary of obstacles.

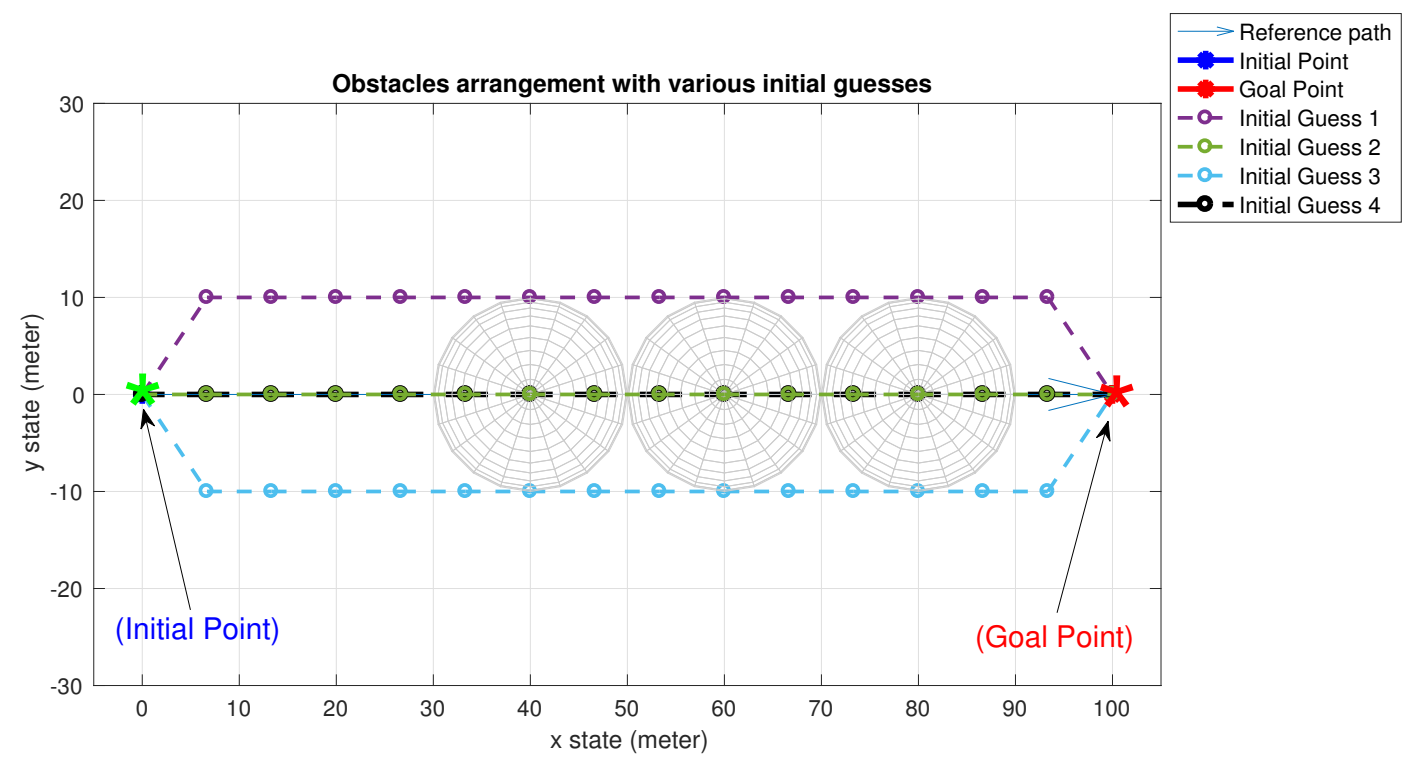

Figure 3.32: Stationary obstacles with various initial guesses, XY view

Similarly, Figure 3.33 shows the $\mathrm{XZ}$ view. The figure shows the initial guesses distri- 
bution where the Initial Guess 3 is distributed 10 meters above the obstacles. Likewise, Initial Guesses 1,2 and 4 are distributed at the height of 10 meters.

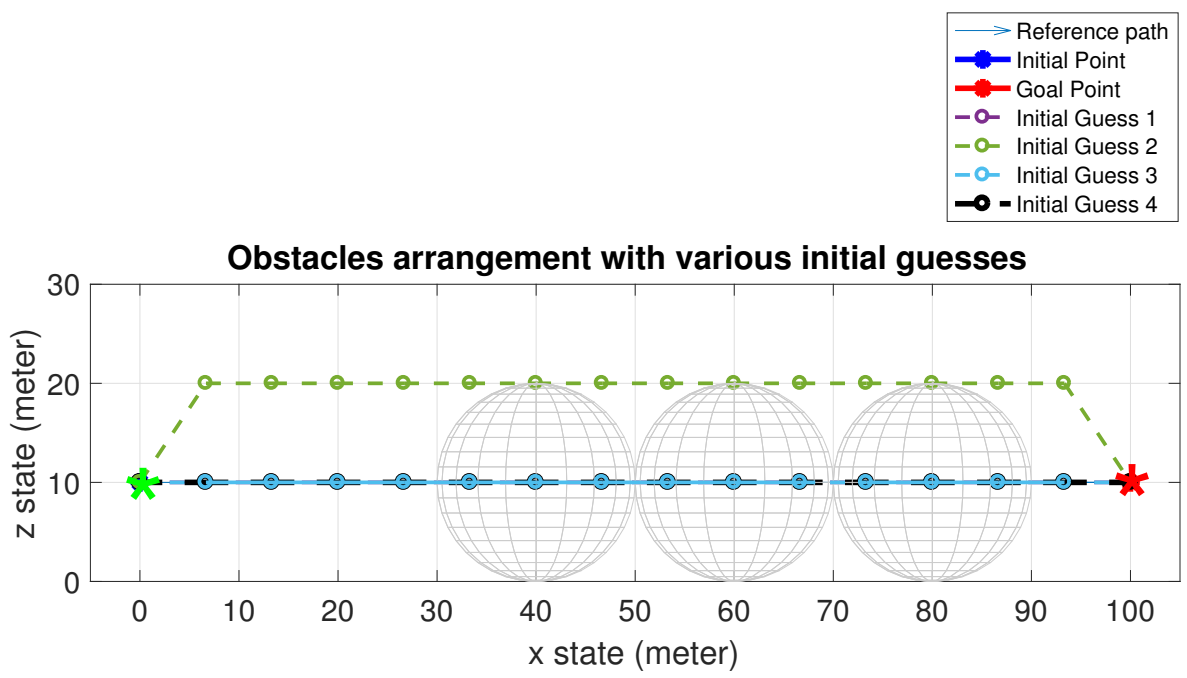

Figure 3.33: Stationary obstacles with various initial guesses, XZ view

Finally, Figure 3.34 shows the arrangement of points in the initial guess with respect to obstacles in $\mathrm{YZ}$ view. As shown in the figure, in all the initial guesses at least some points lie at the boundary of obstacles or inside obstacles boundary.

The infeasible start method (as in 2.2.1) is used to solve the above problems because all the initial guesses have at least a point at the boundary or inside an obstacle. The method is initialized with the value of slack variable as $s=40$, and $\zeta=2$. Then, the solution process of the problems begin, and the sequential convex programming (SCP) iterations are stopped once the value of $s$ goes below $1 \times 10^{-4}$. Since, as similar to examples in this section the problems are initialized with $\epsilon_{s}=0.7$, which means if an sequential convex programming (SCP) iteration solves problem (2.11) and results in the value of $s<0.7$, the solution of the sequential convex programming (SCP) iteration signifies that the trajectory points are outside of an obstacle. Therefore, the sequential convex programming (SCP) iterations could be stopped once $s$ goes below 0.7. Thus, the stopping criteria is chosen as $s=1 \times 10^{-4}$. Moreover, to lower the value of $s$ at each sequential convex programming (SCP) iterations the value of penalty parameter $\zeta$ is increased with multiples of different number and found 2.5 to be the best. Therefore, 


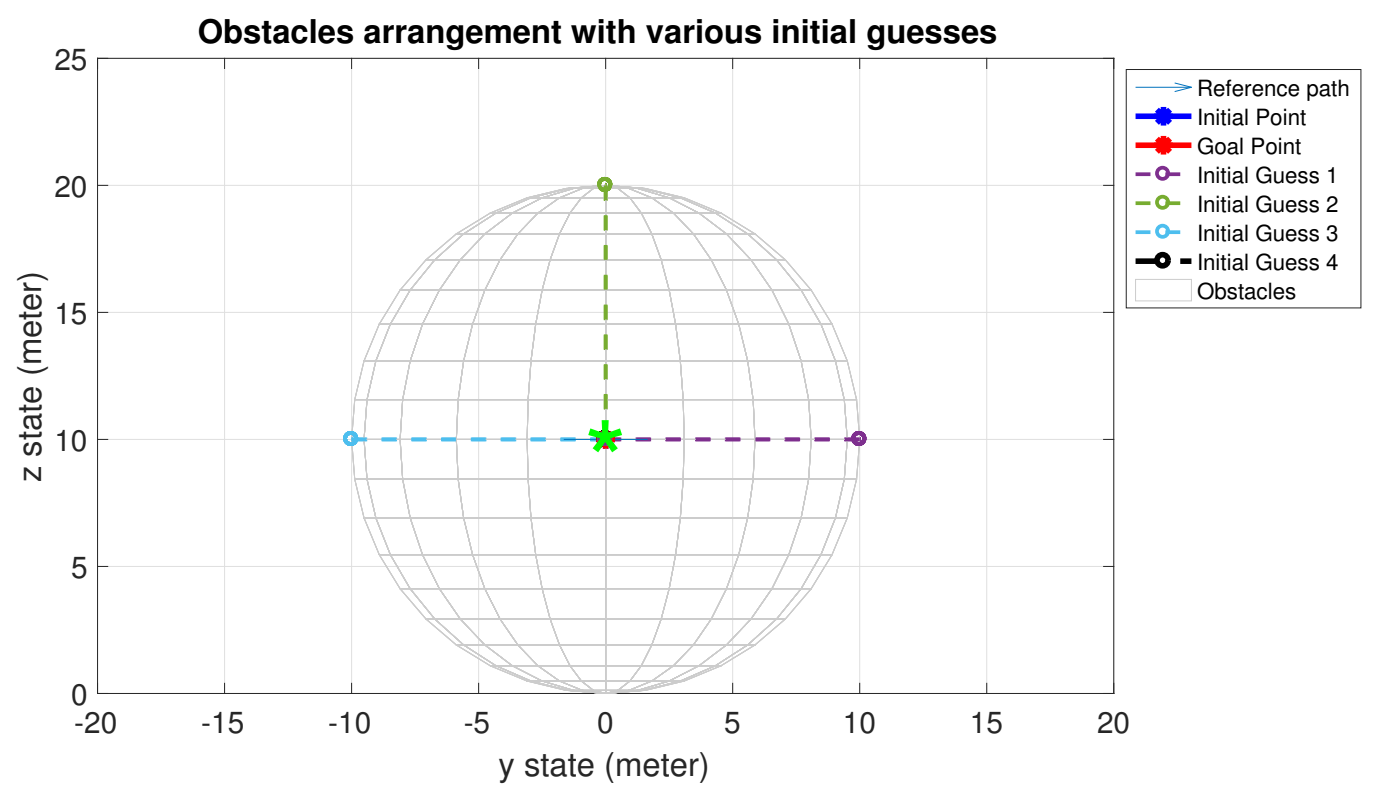

Figure 3.34: Stationary obstacles with various initial guesses, YZ view

the value of $\zeta$ was increased as multiples of 2.5 at each sequential convex programming (SCP) iteration (refer to (2.11)).

Thus, the problems are solved with the above initial guesses and obtain the feasible trajectories as shown in Figure 3.35. The figure shows the feasible trajectories for all the initial guesses with the initial and the goal point, along with a reference path as indicated in the legend. Among the trajectories, it is straight forward to expect the trajectory of Initial Guess 1 to be the left side of the obstacles. However, for the Initial Guess 2, it had equal chances along both the sides, but the solver takes it to the left. It is due to the value of $\epsilon_{I D}$ in (3.45). Likewise, the Initial Guess 4 had equal chances to be on either side of obstacles distribution. However, the Initial Guess 4 takes the right side, which again is due to the value of $\epsilon_{I D}$ which is randomly chosen.

Figure 3.36 shows the $\mathrm{XY}$ view of the resulting feasible trajectories due to initial guesses. It could be observed that the Initial Guesses 1 and 3 are almost symmetric from the reference path. Moreover, both of them take the same number of sequential convex programming (SCP) iterations to arrive at the feasible trajectory, which is shown in Table 3.8. Although feasible trajectories with Initial Guesses 2 and 4 may seem slightly symmetric (they are not), the Initial Guess 2 takes 6 sequential convex programming (SCP) iterations while the Initial Guess 4 takes 4 sequential convex programming (SCP) 


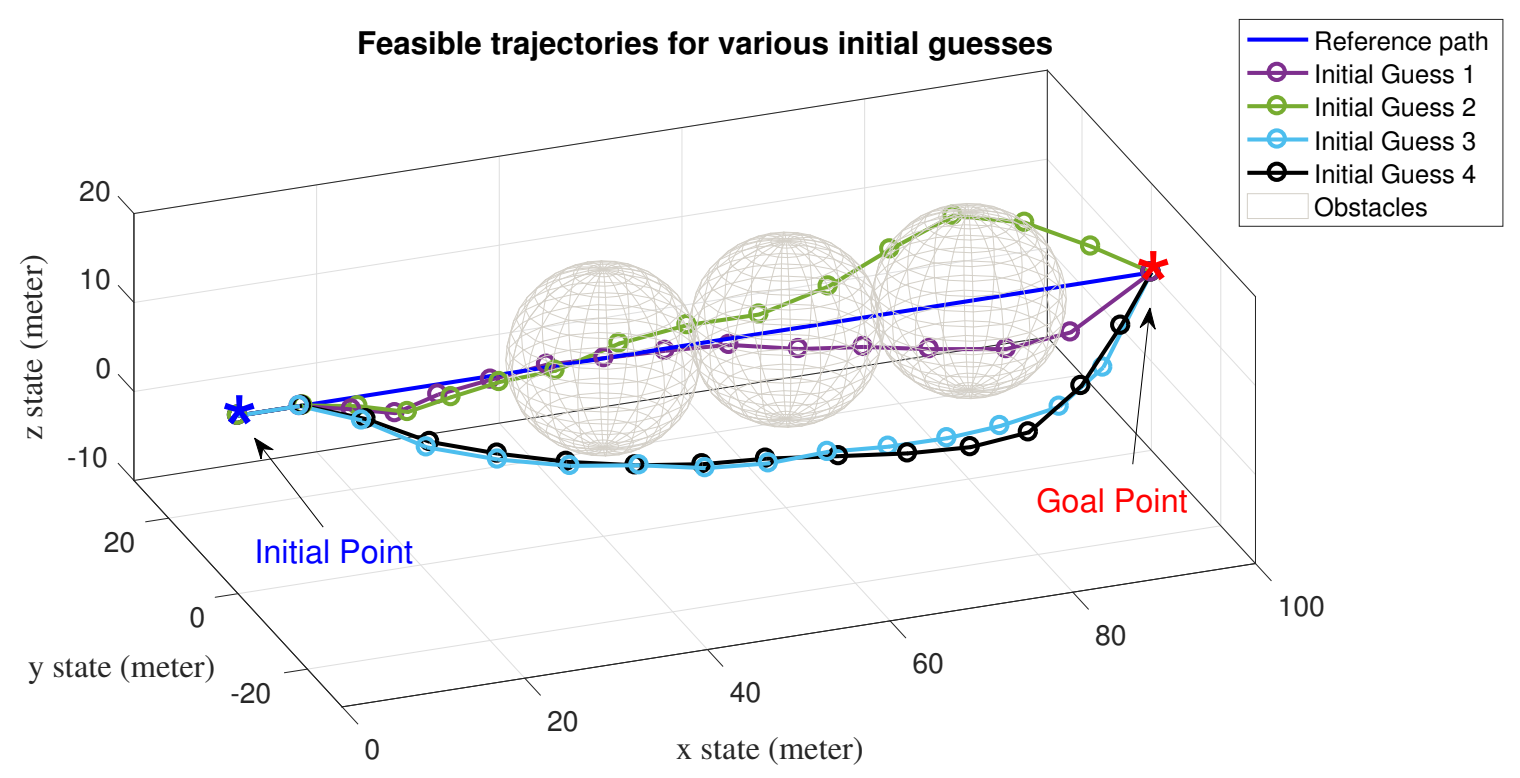

Figure 3.35: Feasible trajectories for the initial guesses, 3D view

iterations to arrive at the feasible trajectory. As the solution procedure is terminated once a feasible solution is obtained (not the optimal solution), the trajectories for the cases 1 to 4 do not have the same value of cost. Therefore, they appear different from each other.

Figures $3.37,3.38$ show the progress of slack variable $(s)$, and the minimum eigenvalue of the overall cost Hessian to a quadratic programming (QP) problem at each sequential convex programming (SCP) iteration for Initial Guesses 1 and 2 respectively. At the beginning of the sequential convex programming (SCP) iteration, $s$ is initialized at 40. In both the figures, the values of $s$ and the minimum eigenvalue of the overall Hessian associated with the sequential convex programming (SCP) at the end of each sequential convex programming $(\mathrm{SCP})$ iteration are presented. It can be seen that for both the cases, the minimum eigenvalue remains almost close to zero while the value of $s$ eventually goes to the terminating value which is $1 \times 10^{-4}$. It takes 3 sequential convex programming (SCP) iterations for $s$ with the Initial Guess 1, while it takes 9 sequential convex programming (SCP) iterations for $s$ with the Initial Guess 2 to reach the terminating value. 


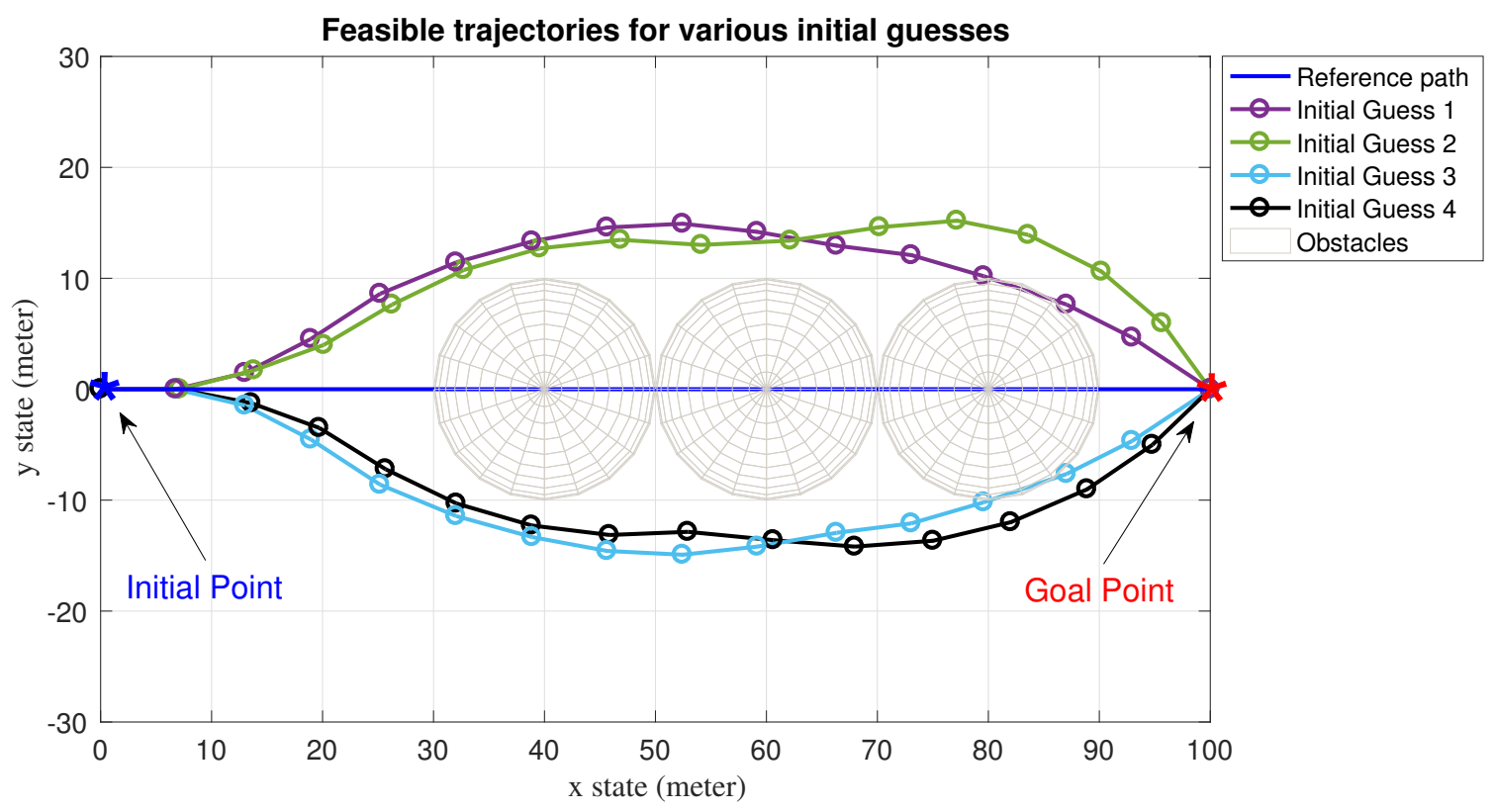

Figure 3.36: Feasible trajectories for the initial guesses, XY view

Similarly, Figures 3.39 and 3.40 show the progress of $s$ and the minimum eigenvalue of the overall Hessian at each sequential convex programming (SCP) iteration for the case of Initial Guesses 3 and 4 respectively. In both the figures, a straight line for the minimum eigenvalue could be observed which is close to zero. Moreover, it takes 3 sequential convex programming (SCP) iterations with the Initial Guess 3, and 4 sequential convex programming (SCP) iterations with the Initial Guess 4 for $s$ to reach the terminating value.

Table 3.8 presents the sequential convex programming (SCP) iterations, the minimum eigenvalue in the iterations, and the solution time for the model problem with the initial guesses. As the custom solver that we use requires that the Hessian of the cost function to the quadratic problem be positive semidefinite, the minimum eigenvalue for the overall cost Hessian of the quadratic problem is a deciding factor. In among the cases, the Initial Guess 2 has the minimum eigenvalue of $-1.694 \times 10^{-10}$, while the Initial Guess 3 has the least solution time with 3 sequential convex programming (SCP) iterations. Whereas, the Initial Guess 2 takes the maximum sequential convex programming (SCP) iteration and the maximum solution time, which are directly related. The minimum eigenvalue 


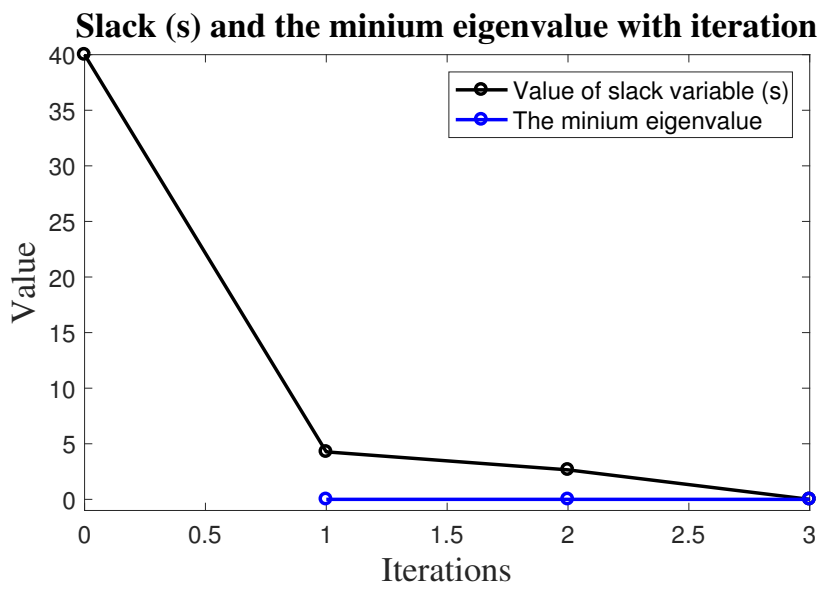

Figure 3.37: The progress of slack (s) and eigenvalue for the Initial Guess 1

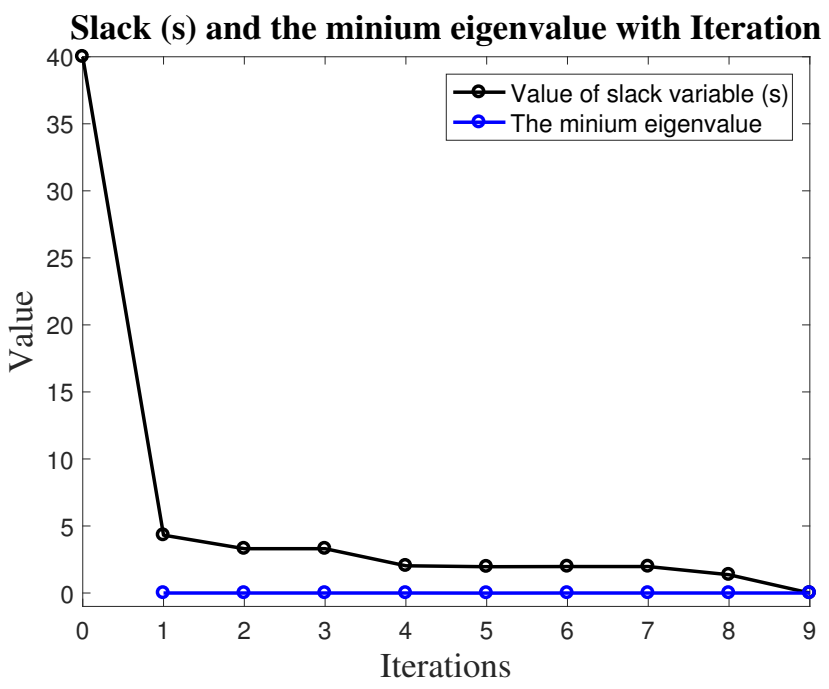

Figure 3.38: The progress of slack (s) and eigenvalue for the Initial Guess 2 


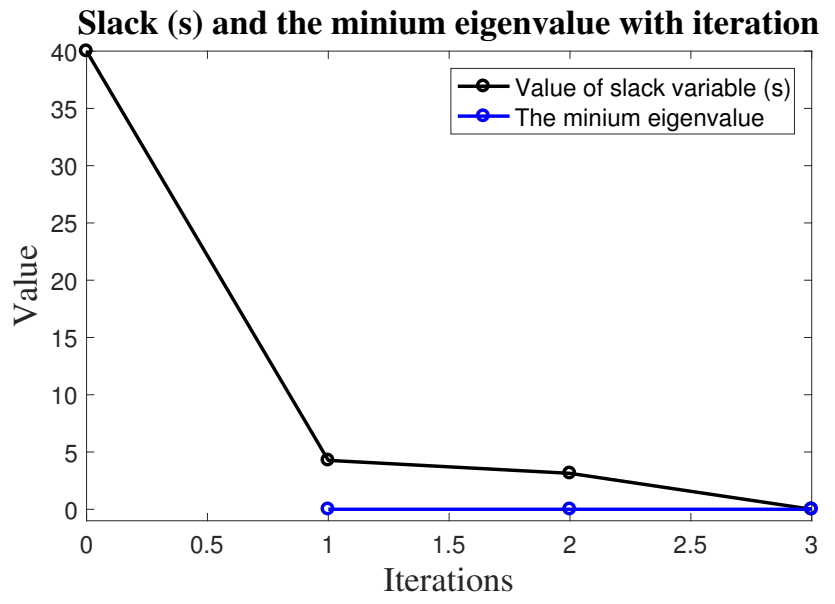

Figure 3.39: The progress of slack (s) and eigenvalue for the Initial Guess 3

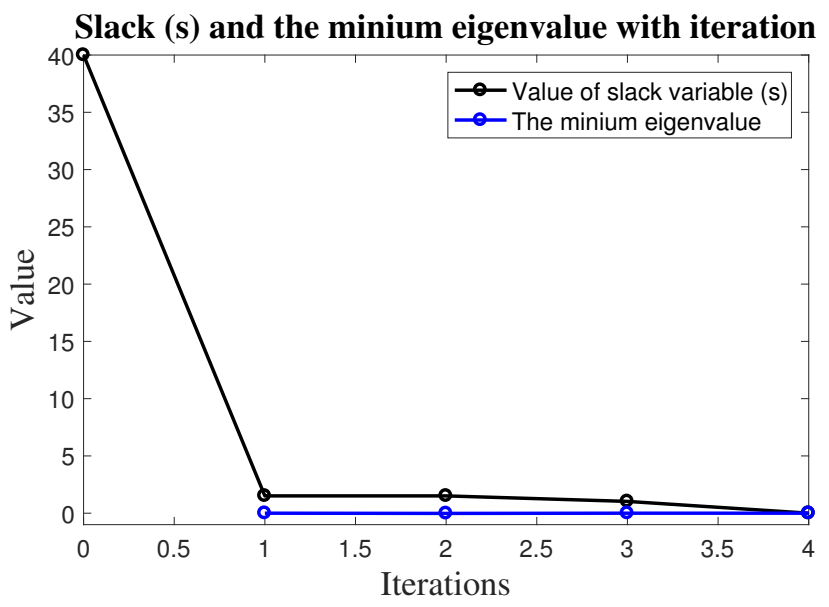

Figure 3.40: The progress of slack (s) and eigenvalue for the Initial Guess 4 
is considered relatively small when compared to with the static regularization (2.20) performed during customization in Chapter 2 which is at the order of $10^{-7}$.

Table 3.8: Iterations and solution time for feasible trajectory

\begin{tabular}{cccc}
\hline \hline Initial guess & SCP iterations & Minimum eigenvalue & Solution time (milliseconds) \\
\hline & & & \\
1 & 3 & $-5.995 \times 10^{-11}$ & 14.25 \\
2 & 9 & $-1.694 \times 10^{-10}$ & 49.90 \\
3 & 3 & $-6.876 \times 10^{-13}$ & 14.14 \\
4 & 4 & $-2.078 \times 10^{-11}$ & 19.28 \\
\hline \hline
\end{tabular}

Hence, even if initial guesses to the trajectory generation problem are provided inside obstacles boundaries, still a feasible trajectory could be found using the approach outlined above. Furthermore, the custom solver could be used for a low solution time.

\subsection{Summary}

An approach for rapid feasible trajectory generation was presented whereby the dynamic equation of a UAV is transformed into a simplified equation from which the bounds on the simplified controls are derived. Then, the states $\gamma, \psi$ and the controls $\omega_{\gamma}, \omega_{\psi}, a$ are transformed as functions of $x, y, z$ points and the velocity $v$. Thus, the transformed problem can be solved iteratively using the sequential convex programming (SCP) algorithm and a custom solver for the quadratic programming (QP) problem. This method applies to the case where the dynamic equation can be simplified to a kinematic model, as shown in Section 3.2.4. The use of a custom solver reduces the computation time to tens of milliseconds for a laptop computer, making the approach applicable for rapid feasible trajectory generation. In addition, the method can be used to find a feasible starting point for solving the original optimal trajectory generation problem, thus reducing the total computation time. 


\section{Chapter 4}

\section{Real-Time Trajectory Generation for Fixed-Wing UAVs}

The Chapter 3 presented a method for rapid generation of feasible trajectory for the case of fixed-wing UAVs with a given desired endpoint. It was observed that the decrease in the optimization variable per point in $3 \mathrm{D}$ space, along with the use of custom solver lowers the solution time of the method to tens of milliseconds. Whereas, this chapter focuses on problems with only a desired flight path, without a goal point, however with an objective to progress forwards along the desired direction of flight. As for real-time implementation, it makes sense to use an model predictive control (MPC) approach, since it allows one to periodically recalculate the trajectory when new information becomes available. Although the approach from the previous chapter could be used within an model predictive control (MPC) framework, the approach requires a desired endpoint. However, in the case considered in this chapter, there is no desired endpoint, so another approach is needed. Next, the typical approaches for trajectory generation use kinematic models, which lead to less capability for performing aggressive obstacle avoidance maneuvers, due to conservatism in bounds on the kinematic parameters (e.g. turning radius), which could be derived from the control bounds in a dynamic model as in chapter 3, or by other means, as in Owlia's thesis [96]. It is therefore desirable to use a dynamic model, and in this chapter, investigation will be made, both in terms of the quality of trajectory obtained, as well as the computational effort required. Therefore, this chapter presents an approach for rapid trajectory generation and autonomous obstacle avoidance for a 
fixed-wing UAV using a dynamic model, and a custom solver to speed up the solution time, and its comparison with a kinematic counterpart. Numerical results show using the proposed method, the dynamic model provides better trajectories compared to the kinematic counterpart, despite having approximately the same computational demands. Also, the method offers low computational requirement for onboard trajectory generation in an embedded platform.

\subsection{Introduction}

Fixed-wing UAVs are preferred for their long endurance ability and low thrust to weight ratio. However, their limits in the minimum turning and pull-up or pull-down radii, and the minimum stall speed constrain them from aggressive maneuvers. These constraints are the result of the UAVs physical design and its dynamics. Moreover, these constraints vary across the flight envelope. Therefore, the use of a dynamic model for a UAVs trajectory generation allows for full utilization of UAV's capabilities, rather than conservatively specifying these constraints up-front as constants and then utilizing a simpler kinematic model. A dynamic model is particularly important when obstacles suddenly appear in front of a UAV, and an aggressive evasive maneuver is necessary. Thus, being able to utilize a dynamic model for a trajectory generation is an asset.

Although a dynamic model with the six degrees of freedom considering a rigid body aircraft incorporates forces, moments, rotation and rotational rates, the equations are complex and may cause a singularity and scaling issues in a numerical solution [26]. Therefore, a reduced-order point mass vehicle model consisting only of a translational equation is often used for the study of real-time trajectory planning [97, 98, 99, 85, 25]. Also, some references use a simple UAV model for path-planning such as in $[100,101,102]$. However, various references was found in the literature that use a dynamic model of a fixed-wing UAV for the study of real-time trajectory generation and obstacle avoidance. References [27, 4] use a dynamic model but lack real-time implementation ability due to large computation time. Reference [38] used a six degrees of freedom UAV model for a study of path-planning using a genetic algorithm. Jian et al. present an analytical method of trajectory planning for fixed-wing vehicles in a dynamic environment [103]. In the method, the family of feasible trajectories are found among the trajectories which 
are parameterized by three polynomials that satisfy boundary conditions, kinematic and the collision avoidance constraints. Then, using a certain performance index a trajectory and its associated steering controls are analytically computed. However, the reference does not explicitly discuss the solution time for the method. Bollino used a high fidelity model for the study of a re-entry vehicle trajectory generation with single digit computation time in seconds [26]; using Pseudospectral discretization and a Nonlinear Programming (NLP) solver [22], and Zhou et al. used a dynamic model to study timeoptimal parameterization of geometric paths [104] and time-optimal path following for fixed-wing UAVs [105]. All of the above references are either focused on offline trajectory generation or do not include detailed discussions on the real-time implementability of the proposed method and the computational complexity associated with the given approach. In [73], Weiran et al. study a multi-stage approach for trajectory generation with rendezvous for UAVs. The stages in the approach consist of, estimation of the path utilizing local $\mathrm{A}^{*}$ algorithm, planning of the path using global $\mathrm{A}^{*}$ algorithm, generation of the trajectory that is flyable using cubic B-spline and finally the modification of the trajectory for rendezvous. Although the reference addresses a different problem than ours the solution time for the method shown uses a desktop computer where only an average solution time is presented. Whereas, for real-time application, the maximum solution time of a method is of interest. Reference [106] studies an interception problem by utilizing the full dynamics of the interceptor. The problem in the reference is posted as a receding horizon control problem and solved directly using a direct optimization technique, where the combination of differential flatness, b-spline curves, and nonlinear programming are used. The maximum solution time for an example using the method shown in the reference is one hundred and sixty three milliseconds using the desktop computer for ten nodes points (with the receding horizon of 2 seconds and the sampling time of 0.2 seconds). Zhao et al. present a nonlinear guidance algorithm for approach and landing of a reusable launch vehicle using the technique of time-varying sliding mode control [107]. Although the reference states the online trajectory generation as a feature of the guidance algorithm, it does not explicitly discuss the solution time associated with the method. Zardashti et al. present a study on trajectory planning for UAVs with a nonlinear model for a multiobjective mission with time-dependent terrain following and threat avoidance [108]. In the reference, Zardashti et al. use a grid-based discrete scheme along with Digital Terrain Elevation Data for the trajectory generation. Although the 
reference attempts to lower the solution time of the overall method, real-time usability of the proposed approach seems remote (in comparison to real-time application for rapid obstacle avoidance) as the solution time for an example shown is 15 seconds. Reference [109] presents the study on rapid path planning for zero-propellant maneuvers of the space station where a solution of a nonlinear programming problem is used to reconstruct a continuous-time feasible solution. Using the method proposed, the computation time for the three different cases shown in the example is in hundreds of milliseconds whereas the computation time for an optimal solution is little over 49 seconds which is far from real-time usable for our case. Although the solution time may be reasonable for the proposed problem, it does not still serve our purpose of real-time trajectory generation and obstacle avoidance. So far, in [49], the author used a custom dynamic model for the study of real-time obstacle avoidance for a fixed-wing UAV. In that reference, the author used a model-based control system that selects a trajectory from a trajectory library in real-time once a UAV detects an obstacle based on the stereo vision technique. The author then uses the Time-Varying Linear Quadratic Regulator to track the selected trajectory in a closed-loop manner. However, a trajectory library requires it to be stored in a memory and accessed repetitively which could consume significant computational resources. Besides, a trajectory library may not contain trajectories for all scenarios.

In order to present a trajectory generation method that is usable for real-time application, we need a context for its usability. A real-time program or a code is one that requires or provides a response within a timing constraint, where a subprogram within the program may keep track of the real-time clock (usually from a hardware independent of processor time) and responds accordingly. Although such details on measuring the time from a real-time clock to assure the real-time usability will not be investigated, the solution time for a problem, and in two different platforms will be provided. The first, is a Macbook laptop with a $1.8 \mathrm{GHz}$ processor and $4 G B$ of $R A M$ and, the second is a Raspberry PI-3B computer [95]. The first platform utilizes an Intel processor whereas the second has an ARM (Advanced RISC Machines, RISC: reduced instruction set computer) processor. Tese two platforms are chosen for the following reasons. The first, based on the size and power consumption Raspberry PI-3B is easily conceivable to be placed onboard a UAV compared to a laptop. The second, a laptop is still the platform for general computation which benefits from low solution time. Kiffmeier presents a brief discussion on real-time hardware-software requirements in [110]. 
The problem of trajectory generation and autonomous obstacle avoidance for a fixedwing UAV is posed as an optimal control problem and then explore methods to approximately solve it. Although contributions by researchers towards indirect methods in the 1950s to the optimal control theory laid a basis for trajectory optimization [17], the use of the minimum principle to solve an optimal control problem by transforming into a two-point boundary value problem is challenging. Likewise, the indirect methods (such as the indirect shooting method, near extreme method and other numerical methods) developed after the 1980s resulted in high sensitivity to initial starting points for the evaluation of a trajectory generation problem ([18], Chapter 7), and challenging for solving complex constraints conditions [19]. Consequently, researchers diverted their attention to the direct iterative approach for solving trajectory optimization problems. Direct methods of trajectory generation involve defining a trajectory generation problem as an optimal control problem, performing some sort of discretization, and solving it as a parameter optimization problem. Thus, to solve our trajectory generation problem, an optimal control problem is transformed to an nonlinear programming (NLP) problem, which is then solved iteratively using a gradient-based nonlinear programming (NLP) algorithm. There are two general classes of approach for solving nonlinear optimal control problems using gradient-based direct optimization techniques, such as [20] and [21]. The first is to discretize the problem and then solve using an nonlinear programming (NLP) technique. It is a discretize-linearize approach, for example, one can use a Pseudospectral Legendre-Gauss-Lobatto method for discretization of the problem [20], and then solve the discretized problem using an nonlinear programming (NLP) algorithm such as SNOPT [22], where SNOPT linearizes the problem internally during the iterative procedure. Using this method, one has to solve a finite-dimensional nonlinear problem. The second method is to convexify the problem and then discretize at specified intervals. For example, one can convexify the problem and then discretize with a fixed time-step. An example of such an approach is the Linear Time-Varying Model Predictive Control [21]. In the second method, one has to solve a discrete convex problem, usually a Quadratic Programming (QP) problem. In this dissertation the second approach (the approach of Model Predictive Control) for trajectory generation is used. It should be noted that the originally posed optimal control problem will no longer be solved, but rather it would be used within an model predictive control (MPC) framework. This is particularly beneficial for real-time trajectory generation, where trajectories need to be updated once 
new environmental information is available. By the use of a gradient-based nonlinear programming (NLP) algorithm, the problem could start with a remote starting point and progress toward an optimal solution. In general, nonlinear programming (NLP) algorithms are computationally intensive, and the rate of convergence may be slow from a remote infeasible starting point to a feasible solution. Therefore, significant efforts are made to lower the computational time by lowering the computational complexity and by using an nonlinear programming (NLP) approach that solves a convex problem to the original problem. One such method that utilizes a convex approximation of an nonlinear programming (NLP) problem to arrive at a solution is the Sequential Convex Programming (SCP) ([31], Page 123). In the sequential convex programming (SCP) method, a general nonlinear programming (NLP) problem is approximated by a convex problem around a given point to find a locally optimal solution, and the process of convexification and solving the convex problem continues until the solution to the original nonlinear programming (NLP) is reached. In this study the sequential convex programming (SCP) method outlined in reference [32] is used, which provides a trust region based sequential convex programming (SCP) to solve an nonlinear programming (NLP) problem (where the convergence guarantee of the sequential convex programming (SCP) with a trust region radius starting from a remote point to an optimal solution is available in [32]). Finally, to solve a sequential convex programming (SCP) problem (a standard quadratic programming (QP) problem) a custom solver is used as discussed in Chapter 2 of this thesis.

Hence, first a trajectory generation problem for a fixed-wing UAV is defined using a dynamic and a kinematic model with an objective to remain close to a desired path while staying away from obstacles. The desired paths are taken to be straight lines, without goal points. Such problems arise in geophysical surveys of a large area, where the area is traced out by a grid of straight lines at a low height for a high-resolution survey. Then, the problem is solved using a Model Predictive Control (MPC) approach [111], where a custom solver is used to solve the sub-problem within the approach.

The remainder of this chapter is arranged as follows. Section 4.2 presents the problem formulation. Section 4.3 details the approach of Finite Horizon Nonlinear Model Predictive Control adopted for our case. Finally, Section 4.4 presents results that include Monte-Carlo test and Section 4.5 summarizes the chapter. 


\subsection{Problem Formulation}

\subsubsection{Dynamic Model for a Fixed-Wing UAV}

In this subsection, a dynamic model for a fixed-wing UAV that includes wind disturbance is introduced. The next subsection presents its kinematic counterpart for comparison purposes. Usually, a high fidelity rigid body model of an aircraft is used in stability and control analysis. However, Feldman [87] suggested that the most detailed model needed in a flight performance problem is a point mass model, as only the motion of a vehicle as a whole is of interest. For our case of comparison between a dynamic and a kinematic model for online trajectory generation, the point mass model of an aircraft is sufficient, since the interest of this comparison lies in understanding the motion (position) of a vehicle for obstacle avoidance at a high level. Therefore, by adding the effect of thrust, a dynamic model of an aircraft derived in references $[88,89]$ and [90] in the presence of a wind field assuming a point mass model, is written as

$$
\left[\begin{array}{c}
\dot{x} \\
\dot{y} \\
\dot{z} \\
\dot{v} \\
\dot{\gamma} \\
\dot{\psi}
\end{array}\right]=\left[\begin{array}{c}
v \cos (\gamma) \cos (\psi)+W_{x}(x, y, z, t) \\
v \cos (\gamma) \sin (\psi)+W_{y}(x, y, z, t) \\
v \sin (\gamma)+W_{z}(x, y, z, t) \\
\frac{1}{M}[T \cos (\alpha)-D-M g \sin (\gamma)]-\dot{W}_{x} \\
\frac{1}{v}\left[\frac{L}{M} \cos (\phi)+\frac{T}{M} \sin (\alpha) \cos (\phi)-g \cos (\gamma)+\dot{W}_{z} \cos (\phi)+\dot{W}_{y} \sin (\phi)\right] \\
\frac{1}{v \cos (\gamma)}\left[\frac{T}{M} \sin (\alpha) \sin (\phi)+\frac{L}{M} \sin (\phi)-\dot{W}_{z} \sin (\phi)-\dot{W}_{y} \cos (\phi)\right]
\end{array}\right]
$$

where $(x, y, z)$ refers to the aircraft position in an inertial frame, $v$ is airspeed, angles $\gamma$ and $\psi$ are the flight path angle and the heading angle of the velocity vector relative to the air. Thrust $(T)$, angle of attack $(\alpha)$, and roll angle $(\phi)$ are treated as control inputs, $\left(W_{x}, W_{y}, W_{z}\right)$ represent wind fields along the $x, y$ and $z$ axes respectively, $M$ is mass of the aircraft, $L$ (lift force) $=\frac{1}{2} \rho v^{2} S C_{L}, D$ (Drag force) $=\frac{1}{2} \rho v^{2} S C_{D}, g$ is an acceleration due to gravity $\left(9.81 \mathrm{~m} / \mathrm{s}^{2}\right)$, the coefficient of drag, $C_{D}=C_{D_{0}}+K C_{L}^{2}$, coefficient of lift, $C_{L}=C_{L_{\alpha}} \alpha$, and $K=\frac{1}{\pi A e}$. The wing surface area is $S, A$ is aircrafts aspect ratio given by $A=\frac{b^{2}}{S}$, where $b$ is the wing span, $e$ is an Oswald efficiency, and $\rho$ is air density.

While $\alpha, \phi$ and $T$ are treated as control inputs to the dynamic model (4.1), using 
them directly in a parameter optimization problem would allow these controls to jump between their limits. Therefore, it is necessary to be able to limit their rates of change. However, these do not act as control inputs to a UAV, but rather a trajectory generated with these control constraints is send to an autopilot for tracking the trajectory (see in Figure 1.5). Thus, they $(\alpha, \phi, T)$ are augmented along with the state vector, treating their rates as control inputs. Hence new terms are introduced, the rate of change of angle of attack as $\omega_{\alpha}$, the rate of change of roll angle as $\omega_{\phi}$, and the rate of change of Thrust as $T_{r}$, such that

$$
\left[\begin{array}{c}
\dot{\alpha} \\
\dot{\phi} \\
\dot{T}
\end{array}\right]=\left[\begin{array}{c}
\omega_{\alpha} \\
\omega_{\phi} \\
T_{r}
\end{array}\right]
$$

After augmenting the relation (4.2) to the equation (4.1), we have new state variables as $(x, y, z, \gamma, \psi, v, \alpha, \phi, T)$, and control as $\left(\omega_{\alpha}, \omega_{\phi}, T_{r}\right)$. For a fixed-wing UAV, there are limits on the state and the control variables within which a UAV can perform. For example, $v$ is limited from below by a positive stall speed, and from above by the maximum speed the UAV's structure can withstand. Thus, the limits on the states and the controls are represented by over and under bars respectively given by

$$
\underline{q} \leq q \leq \bar{q},
$$

where $q$ represents each state and control variable.

\subsubsection{Kinematic Model for a Fixed-Wing UAV}

To be consistent with the dynamic model in Section 4.2.1, a kinematic model used for the path planning purpose in [91] is taken and then appended it with a wind field model as follows. 


$$
\left[\begin{array}{c}
\dot{x} \\
\dot{y} \\
\dot{z} \\
\dot{\gamma} \\
\dot{\psi} \\
\dot{v}
\end{array}\right]=\left[\begin{array}{c}
v \cos (\gamma) \cos (\psi)+W_{x}(x, y, z, t) \\
v \cos (\gamma) \sin (\psi)+W_{y}(x, y, z, t) \\
v \sin (\gamma)+W_{z}(x, y, z, t) \\
\omega_{\gamma} \\
\omega_{\psi} \\
a
\end{array}\right]
$$

where $\omega_{\gamma}$ (rate of change of flight path angle), $\omega_{\psi}$ (rate of change of heading angle) and $a$ (acceleration) are treated as controls. The dynamic trajectory constraints resulting from constraining thrust $(T)$ carries over directly as a constraint on $a$ (acceleration), but the constraints to $\alpha$ and $\phi$ cannot be directly carried over to the kinematic model. Instead, constraints are made on the turning and pull-up radii, which translate to constraints on the turning rates. Therefore, constraints on the control variables take the form [3],

$$
C=\left\{\begin{array}{cc}
\omega_{\gamma}: & -\frac{1}{R_{\min }^{P}} \leq \frac{\omega_{\gamma}}{v} \leq \frac{1}{R_{\min }^{P}} \\
\omega_{\psi}: & -\frac{1}{R_{\min }^{T}} \leq \frac{\omega_{\psi}}{v} \leq \frac{1}{R_{\min }^{T}} \\
a: & \underline{a} \leq a \leq \bar{a}
\end{array}\right\} \subset \mathbb{R}^{3}
$$

where $R_{\min }^{P}$ is the minimum pull-up or pull-down radius, $R_{\min }^{T}$ is the minimum turning radius, and $\underline{a}=\underline{\mathrm{T}} / M$, and $\bar{a}=\bar{T} / M$ are the minimum and the maximum allowable acceleration $(a)$ for an aircraft respectively.

The minimum pull-up or pull-down radius is calculated a-priori using equation $R_{\min }^{P}=$ $\frac{\bar{v}^{2}}{g(n-1)}$ [112]. Similarly, the minimum turning radius is calculated using equation $R_{\text {min }}^{T}=$ $\frac{\bar{v}^{2}}{g \sqrt{n^{2}-1}}$, where $n=\frac{L}{W}$ is the load factor of an aircraft defined as the ratio of lift $(L)$ to weight $(W)$. For the purpose of trajectory generation, while conservative, it is computationally convenient to have a constant $R_{\min }^{T}$, which requires a constant load factor $n$. We can use either the maximum load factor from the $L / W$ ratio or the maximum allowable load factor during roll. In the case of turning flight, $n=\frac{1}{\cos (\phi)}$ for calculating the load factor [3], where $\phi$ is the roll angle. Thus to calculate the maximum load factor the maximum allowable roll angle is taken, which is $\bar{n}=\frac{1}{\cos (\bar{\phi})}$. Also, the maximum load factor is calculated from the relation $\bar{n}=\frac{\bar{L}}{W}$, where $\bar{L}$ can be calculated from the maximum allowable velocity $\bar{v}$. Finally, the load factor that is the minimum between these two options is chosen, so as not to exceed the limit of load factor from one crite- 
rion due to the other option during maneuver, thus $\bar{n}_{s}=\min \left\{\frac{1}{\cos (\bar{\phi})}, \quad \frac{\bar{L}}{W}\right\}$, and hence calculate the minimum turning and pull-up radii as $R_{\min }^{T}=\frac{\bar{v}^{2}}{g \sqrt{\bar{n}_{s}^{2}-1}}$ and $R_{\min }^{P}=\frac{\bar{v}^{2}}{g\left(\bar{n}_{s}-1\right)}$ respectively.

Moreover, for the same reason of adding (4.2) to model (4.1), the kinematic model (4.4) is augmented with control rates, so as to limit the rate of change of controls $\left(\omega_{\gamma}, \omega_{\psi}, a\right)$ for a trajectory.

Thus,

$$
\left[\begin{array}{c}
\dot{\omega}_{\gamma} \\
\dot{\omega}_{\psi} \\
\dot{a}
\end{array}\right]=\left[\begin{array}{c}
\omega_{\gamma_{r}} \\
\omega_{\psi_{r}} \\
a_{r}
\end{array}\right]
$$

where $\omega_{\gamma_{r}}, \omega_{\psi_{r}}, a_{r}$ are the rates of change of $\omega_{\gamma}, \omega_{\psi}, a$ respectively. After augmenting equation (4.4) with (4.6), we now have new state variables $\left(x, y, z, \psi, \gamma, v, \omega_{\gamma}, \omega_{\psi}, a\right)$, and control inputs as $\left(\omega_{\gamma_{r}}, \omega_{\psi_{r}}, a_{r}\right)$ for the kinematic model.

As in Section 4.2.1, there are limits on the state variables and saturation in the control as in (4.3). Also, as the turning and pull-up radii constraints on the kinematic model are inherently conservative, so it is expected that the dynamic model will be able to generate more aggressive trajectories compared to the kinematic model.

\subsubsection{Problem Definition}

A trajectory generation problem for a fixed-wing UAV is considered in which the UAV has to avoid obstacles while remaining close to a desired reference path. The desired reference path is chosen such that it extends from the origin of a suitably defined reference frame along the positive $x$-axis, at a desired height $z_{d}$. It should be noted that many practical reference paths are made up of connected straight line segments, and any straight horizontal path can be mapped into this line by simple translation and rotation of coordinates. Then, the trajectory generation problem is posed as an optimal control problem where an objective is to minimize a cost function subject to a kinematic or a dynamic constraint, obstacle constraint, and state and control saturations. In order to remain close to the positive $x$ - axis at a desired height a running cost that minimizes the square of the state $y(t)$ and the term $\left(z(t)-z_{d}\right)^{2}$ are used, where $z_{d}$ is the desired height along the $z$-axis. In order to progress along the $x$-axis, the term $\left(x(t)-x_{a}\right)^{2}$ is 
added to the cost function where the term minimizes the square of the difference between an arbitrary point $x_{a}$ and the state $x(t)$ of a UAV. The point $x_{a}$ is defined far enough such that the final state of a UAV can never reach the point, within a given final time $t_{f}$ (is fixed). Since, the problem does not have a goal point, $x_{a}$ is introduced far away along the desired direction of flight, such that the cost associated with it remains high, and it is a significant factor driving the UAV in the desired direction of flight. In order to avoid obstacles, the obstacle model is taken from [4], where an avoidance condition corresponding to the $i^{\text {th }}$ obstacle is represented by

$$
h_{i}(x, y, z)=\ln \left[\left|\left(\frac{x-x_{c i(t)}}{a_{i}}\right)\right|^{p_{x_{i}}}+\left|\left(\frac{y-y_{c i(t)}}{b_{i}}\right)\right|^{p_{y_{i}}}+\left|\left(\frac{z-z_{c i(t)}}{c_{i}}\right)\right|^{p_{z_{i}}}\right] \geq \epsilon>0
$$

where $x_{c i(t)}, y_{c i(t)}, z_{c i(t)}$ represent the inertial coordinates of the centre of obstacle $i$, for $i=1, \ldots, n$, where $n$ is the number of obstacles, $p_{x_{i}}, p_{y_{i}}, p_{z_{i}} \geq 2$ are exponent terms determining the shape of the obstacle, and $a_{i}, b_{i}, c_{i}$ represent the obstacle's radius from its centre along the $x, y, z$ axes respectively. In the case of a moving obstacle, the location of the centre is time-dependent. The constant $\epsilon$ is used to ensure that the feasible region for the UAV is closed. However $\epsilon$ can also be viewed as imposing a buffer distance between a UAV and an obstacle. To ensure the UAV's safety from an obstacle and to increase the robustness in obstacle avoidance, a robustness function is augmented to the cost, similar to reference [25], and write as,

$$
\bar{r}(x, y, z)=\sum_{i=1}^{n} w_{i}(x, y, z)\left[e^{e^{\left(\epsilon-\overline{h_{i}}(x, y, z)\right)}}-1\right]
$$

where

$$
\begin{gathered}
w_{i}(x, y, z)=q \cdot g\left(\left[\left(\frac{x-x_{c i(t)}}{r_{i}}\right)^{2}+\left(\frac{y-y_{c i(t)}}{r_{i}}\right)^{2}+\left(\frac{z-z_{c i(t)}}{r_{i}}\right)^{2}\right]\right) \\
h_{i}(x, y, z)=\ln \left[\left(\frac{x-x_{c i(t)}}{r_{i}}\right)^{2}+\left(\frac{y-y_{c i(t)}}{r_{i}}\right)^{2}+\left(\frac{z-z_{c i(t)}}{r_{i}}\right)^{2}\right]
\end{gathered}
$$

where $r_{i}=\max \left(a_{i}, b_{i}, c_{i}\right)$. In equation $(4.9) q>0$, and $g(\cdot)$ is defined as, 


$$
g\left(t_{d}\right)=\frac{f\left(r_{b}-t_{d}\right)}{f\left(r_{b}-t_{d}\right)+f\left(t_{d}-r_{a}\right)}
$$

for suitably chosen $r_{a}<r_{b}, t_{d} \in(-\infty, \infty)$, and $f\left(t_{d}\right)$ is defined as,

$$
f\left(t_{d}\right)= \begin{cases}0 & t_{d} \in(-\infty, 0] \\ \exp \left(-1 / t_{d}\right), & t_{d} \in(0, \infty)\end{cases}
$$

This function is $\mathcal{C}^{\infty}$, and its derivative is given by

$$
d f\left(t_{d}\right) / d t_{d}= \begin{cases}0 & t_{d} \in(-\infty, 0] \\ \left(1 / t_{d}^{2}\right) \exp \left(-1 / t_{d}\right), & t_{d} \in(0, \infty)\end{cases}
$$

$g\left(t_{d}\right)=1$ is obtained for $t_{d} \leq r_{a}$, and $g\left(t_{d}\right)=0$ is obtained for $t_{d} \geq r_{b}$. Since for $t_{d}<1$ corresponds to points inside an obstacle, we require $1<r_{a}<r_{b}$, to ensure the weight is non-zero in a region outside the obstacle.

Figure 4.1 shows the function $g\left(t_{d}\right)$ with $r_{a}=1$ and $r_{b}=2$ while $t_{d} \in[0,3]$. Since $t_{d}$ in this case is the distance between an obstacle and the UAV, the weight on robustness function goes to maximum $(q \cdot 1=q)$ when the UAV approaches the obstacle, and the weight goes to the minimum $(q \cdot 0=0)$ when an obstacle is far than $r_{b}$ in distance. Thus, the definition of the weight (in equation (4.9)) ensures that the obstacles far $\left(>r_{b}\right)$ from the UAV do not affect the resulting flight path. This is a major difference from the robustness function in [25].

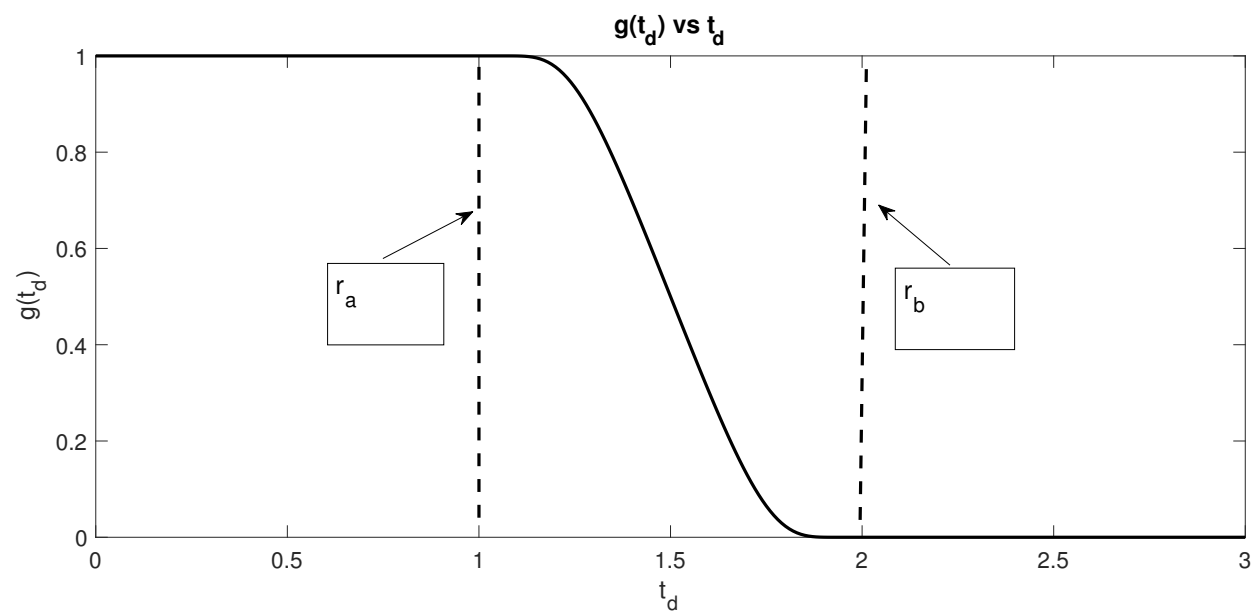

Figure 4.1: The value of $g(t)$ for obstacle robustness factor 
Hence, by introducing penalty terms associated with different cost (that we have chosen), the overall cost function over the time interval $\left[t_{0}, t_{f}\right]$ is written as

$$
J(X(\cdot))=\int_{t_{0}}^{t_{f}}\left[\eta\left(x(t)-x_{a}\right)^{2}+\alpha y(t)^{2}+\beta\left(z(t)-z_{d}\right)^{2}+\bar{r}(x, y, z)\right] d t
$$

where $\alpha>0, \beta>0$ and $\eta>0$ are penalty parameters.

Although it has been stated to use a convex solver in the introduction of this chapter, the overall cost function (4.14) is non-convex. Therefore, we may not be strictly solving a convex problem always, especially near an obstacle boundary (due to the effect of the robustness function the Hessian of the cost function may turns out to be indefinite). Besides, the starting point for the UAV may not satisfy the dynamics and obstacle constraints. Thus, to deal with the cases when the cost function is non-convex near or inside an obstacle boundary, a method that is similar to the infeasible start Newton method ([62], Chapter 10.3) is used to arrive at a feasible solution. Also, the infeasibility due to obstacles and dynamics constraints is not an issue for convergence, which will be apparent later during the solution procedure. For subsequent solves of the trajectory generation problem, our model predictive control method uses the tail end of the previous optimal trajectory as part of the initial guess for the next trajectory generation solution. It is reasonable to expect that all initial guesses therefore remain at least close to optimal, with the cost function remaining locally convex. Indeed, in all iterations of all cases tried in the Numerical example section 4.4, the minimum eigenvalue of the cost function Hessian was a very small positive number. As such, the sequential convex programming (SCP) approach works very well for the trajectory generation problem as posed in this chapter.

\subsubsection{Problem Summary}

The trajectory generation problem for obstacle avoidance posed as an optimal control problem for a fixed-wing UAV using both the dynamic and the kinematic models are as follows.

Given initial condition $X_{0}$, find the control-state pair $(X(t), U(t))$ to minimize cost (4.14) subject to 


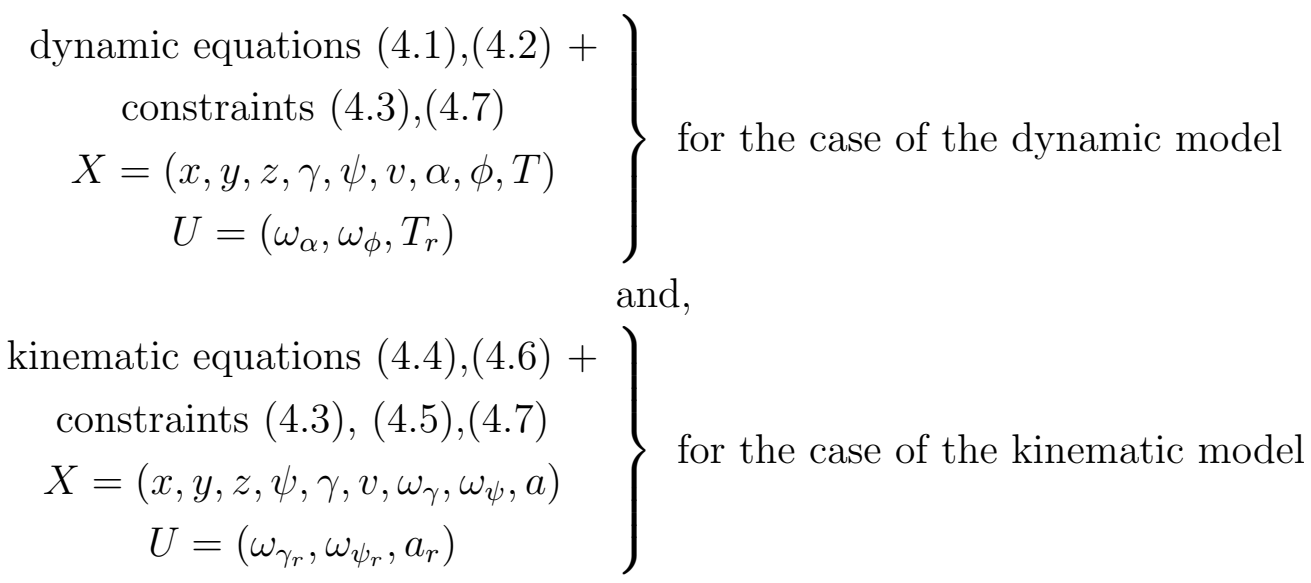

It should be noted that the wind field in (4.1) and (4.4) is not included in the above optimal control problem formulation, since it is considered a disturbance and therefore not known by the on-board flight computer. Although equations (4.1) and (4.4) contain the wind velocity terms, these are not used in solving the optimal control problem but are used to evaluate the real trajectory resulting from integrating the generated optimal controls to the real systems in (4.1) and (4.4) respectively.

\subsection{Finite Horizon Model Predictive Control Prob- lem}

Ideally, it was desired to solve the optimal control problem (4.15). As stated in the introduction of this chapter, using a general nonlinear programming (NLP) method to solve the problem (4.15) is computationally costly; therefore an approach of using the sequential convex programming (SCP) method together with a custom solver was chosen to solve the convex problem obtained in a sequential convex programming (SCP) iteration. Regarding the implementation of the generated controls on a UAV, it is not necessary to have the entire trajectory available, instead just a portion of the trajectory over an immediate short window is enough. Besides, the appearance of any new obstacles during flight necessitates re-solving the trajectory generation problem. Therefore, a Finite Horizon Model Predictive Control approach [21] is used, with the same constraints and the same cost function as defined in Section 4.2. To this end, the formulated optimal control 
problem in (4.15) is utilized within the following Nonlinear Model Predictive Control (NMPC) framework.

$$
\begin{array}{cc}
\text { Minimize } & J(Z(\cdot))=\int_{t}^{t+H_{p}} F(X(\tau), U(\tau)) d \tau \\
\text { subject to } & \dot{X}(\tau)=f(X(\tau), U(\tau)) \\
g(X(\tau), \tau) \geq 0 \\
X(t)=X_{t} \\
\underline{X} \leq X(\tau) \leq \bar{X} \\
\underline{U} \leq U(\tau) \leq \bar{U} \\
\tau \in\left[t, t+H_{p}\right]
\end{array}
$$

where $H_{p}$ is a fixed finite horizon, $t$ is the current time, and $Z(\cdot)=(X(\cdot), U(\cdot))$. In comparison with problem (4.15), $F(X(\tau), U(\tau))$ represents the running cost as in (4.14), $\dot{X}(\tau)=f(X(\tau), U(\tau))$ represents the kinematic or the dynamic equation, and $g(X(\tau), \tau)$ represents the inequality constraint due to obstacles as in (4.7). The bounds on the states and the controls remain as they are, given by the over and under bars on states and controls respectively. Let $U(\tau)^{*}$ represent an optimal control solution for finite horizon problem (4.16), then at each instant $(t)$, the finite horizon nonlinear model predictive control (MPC) controller implements $U(\tau)=U(\tau)^{*}$ up to a control horizon $t+t_{c}$, where $0<t_{c}<H_{p}$, and the remaining unimplemented controls are used as nominal controls for the next nonlinear model predictive control (MPC) time window. In terms of nonlinear model predictive control (MPC) stability, since there is no end-point cost or constraint, the standard methods of establishing closed-loop stability do not apply [30]. However, Marcus Reble [113] demonstrated that under certain controllability conditions, provided the prediction horizon is long enough compared to the control window, the model predictive control (MPC) trajectory converges even without an endpoint cost. More recently, Alexander Joos has presented a case-specific example by calculating a sufficient finite horizon yielding asymptotic stability of Nonlinear model predictive control (MPC) problem for a fixed-wing UAV [114]. However, the focus of this chapter is on the computational aspects of trajectory generation, thus a theoretical stability of the stated nonlinear model predictive control (MPC) problem will not be investigated, but will be demonstrated numerically.

The problem (4.16) is a finite horizon non-convex problem which will be solved using 
the sequential convex programming (SCP) approach. A summary on the transformation from a Nonlinear Finite Horizon optimal control problems to convex parameter optimization problems can be found in various references, such as $[115,116]$. The problem (4.16) is convexified by taking a first-order Taylor approximation of the nonlinear UAVs system equations and constraints, and a second-order Taylor approximation of the cost function about a nominal state-control pair. A brief discussion on the nominal state-control pair is presented below. After the approximation, the problem becomes a finite horizon convex problem with a quadratic cost function. Then, the convexified problem is discretized at fixed-time steps up to a finite horizon $t+H_{p}$, where zero-order hold is assumed for the controls in-between sample times. Finally, a discrete convex parameter optimization problem is obtained, where the collection of all the states and controls at discrete points are optimization parameters. The resulting convex optimization problem takes the form

$$
\begin{gathered}
\tilde{J}(\tilde{Z})=\frac{1}{2} \tilde{Z}^{T} H \tilde{Z}+R^{T} \tilde{Z} \\
\text { Minimize } \\
X_{\tau+\Delta t}=A_{d}^{\tau} X_{\tau}+B_{d}^{\tau} U_{\tau}+g_{d}^{\tau}, \quad(\tau \in\{t, t+\Delta t, \ldots, t+N \Delta t\}) \\
g_{x}^{\tau} X_{\tau} \geq g_{c}^{\tau} \\
X(t)=X_{t} \\
\underline{X} \leq X(\tau) \leq \bar{X} \\
\underline{U} \leq U(\tau) \leq \bar{U} \\
\tilde{Z}=\left(X_{t}, U_{t}, X_{t+\Delta t}, U_{t+\Delta t}, \ldots . ., U_{t+(N-1) \Delta t}, X_{t+N \Delta t}\right)
\end{gathered}
$$

where $H$ is the Hessian, $R$ is gradient, $A_{d}^{\tau}$ and $B_{d}^{\tau}$ are the Jacobians of UAVs system equations with respect to the states and the controls respectively, $g_{d}^{\tau}$ is constant term of UAVs system equations after the first-order Taylor approximation, $g_{x}^{\tau}$ is the Jacobian of the obstacle constraint with respect to the states, and $g_{c}^{\tau}$ is a constant term for obstacle constraint at time instant $\tau$ after the first-order Taylor approximation, $t$ is the current time, $X_{t}$ is the current state, $U_{t}$ is the control at the current time $t, N$ is the number of steps (or discrete node points) and $\Delta t$ is the length of each time step. The Appendix 3 provides a brief note on the convexification.

At the first time instant $(t=0)$, nominal states are defined as the current state $\left(X_{\tau}=X_{0}\right)$ for $\tau \in\{0, \Delta t, \ldots, N \Delta t\}$, and nominal control as zeros $\left(U_{\tau}=0\right)$ for $\tau \in$ $\{0, \Delta t, \ldots,(N-1) \Delta t\}$ but at the subsequent time instants $(t>0)$ the predicted state- 
control pair is defined as the nominal pair. In the beginning, $t=0$, the nominal statecontrol pairs taken may not satisfy the dynamics and obstacle constraints. Thus, the initial nominal pair may not be feasible. However, it is apparent from Chapter 2 that this is not an issue for convergence to a feasible solution. Once nominal points are available at $t=0$, the problem (4.17) can be obtained. Then, the sequential convex programming (SCP) method from [32] is used to solve the problem (4.16), where at each sequential convex programming (SCP) iteration the problem (4.17) is solved. Such infeasibility arising from obstacle constraint is handled by the quadratic programming (QP) solution strategy, as discussed in Section 2.3. However, in case the problem (4.16) turns out to have an indefinite Hessian due to the effect of the robustness function, a method similar to the infeasible start Newton method ([62], Chapter 10.3) is used to arrive at an obstacle free solution, also summarized in Section 2.2.1.

In the following the solution procedure is summarized. At the time $(t=0)$, the model predictive control (MPC) algorithm is initialized by setting $\left\{X_{0}, X_{1}, X_{2}, \ldots, X_{N}\right\}$ and $\left\{U_{0}, U_{1}, \ldots, U_{N-1}\right\}$ as the sets of nominal states and controls, respectively, where at $t=0, X_{i}=X_{0}$ for $i=1, \ldots N$ and $U_{j}=0$ for $j=1, . . N-1$. Then, the problem (4.17) is solved to obtain controls. Let the controls which are the solutions to the problem (4.17) be $U_{0}^{*}, U_{1}^{*}, \ldots, U_{N-1}^{*}$, and the predicted states be $X_{0}^{*}, X_{1}^{*}, X_{2}^{*}, \ldots, X_{N}^{*}$. Subsequently the controls $U_{0}^{*}, \ldots, U_{k}^{*}$ are implemented into the continuous system through zero-order hold, where $0 \leq k<N$, is the discrete control horizon. Thus, implementing controls $U_{0}^{*}, . . U_{k}^{*}$, the UAV reaches state $\bar{X}_{k+1}$, then $\bar{X}_{k+1}$ is updated as a current state for the next model predictive control (MPC) iterate. Now the unimplemented controls and the predicted states $\left\{U_{k+1}^{*}, \ldots, U_{N-1}^{*}\right\}$, and $\left\{X_{k+2}^{*}, \ldots, X_{N}^{*}\right\}$ respectively act as the nominal state-control pair for the next model predictive control (MPC) iteration, with the addition of new nominal controls as $\left\{U_{N}, \ldots, U_{(N+k-1)}\right\}=\{0\}$ (to adjust the number of nodes) and the new nominal states corresponding to these newly added controls obtained by integrating the newly added nominal controls to the system model starting from $X_{N}^{*}$.

Then at $t>0$, problem (4.17) is solved with the nominal state-control pair that gets updated at each model predictive control (MPC) iteration as stated above, and the process repeats. 


\subsection{Numerical Examples}

\subsubsection{Scenario with Two Obstacles}

To illustrate the proposed method, an example is considered that has a stationary and a moving obstacle. To add a disturbance to the problem of remaining close to a reference path a wind effect is added along the negative $y$-axis that is perpendicular to the desired reference path. The stationary obstacle is represented by a cylindrical shaped structure situated at $(300,20)$ meter with radius of 50 meter and the height of 100 meter. The constant wind field has a speed of $20 \mathrm{~km} / \mathrm{h}$ or approximately $5.55 \mathrm{~m} / \mathrm{s}$ and acts between $x=700$ meter to $x=1200$ meter. A moving obstacle moves towards the negative $y$-axis with its centre starting at $(900,300,50)$ meter such that the obstacle starts moving when the UAV reaches $x=600$ meter. The moving obstacle is represented by a spherical structure of radius 30 meter. The moving obstacle moves such that the $y$ coordinate of the center of the obstacle changes according to the center of the $x$ coordinate of the UAV, given by

$$
y_{o b s}^{m, t}=y_{o b s}^{m, 0}+600-x_{u a v}^{t}, \text { if } x_{u a v}^{t} \geq 600
$$

where, $y_{o b s}^{m, t}$ is the centre of the $y$ coordinate of the moving obstacle at time $t, y_{o b s}^{m, 0}=300$ meter is the starting point of the moving obstacle, and $x_{u a v}^{t}$ is the location of the UAV at time $t$. With this definition, the UAV will collide with the moving obstacle unless an evasive maneuver is performed. Figure 4.2 shows the problem scenario which includes obstacles, the reference path, and the wind field. The moving obstacle moves along the broken line at $x=900$ meter. In the same figure, there are two arrows, one at $x=700$ meter and next at $x=1200$ meter, they represent the region in-between which the UAV will experience the wind effect. It is to be noted that the effect of wind is not known for the purposes of trajectory generation but is experienced as a disturbance. Table 4.1 shows the nature of wind under consideration.

The UAV starts from $(x=0, y=0, z=50)$ meter with velocity $(v=10 \mathrm{~m} / \mathrm{s})$ along the positive $x$-axis, and the desired reference path extends to $x=1200$ meter along the $x$-axis at the height of $z_{d}=50$ meter. For numerical examples, the parameters of a fixed-wing electric powered UAV (Ascent [1]) have been used from the Table 3.1. Table 4.2 presents the lower and upper bounds on the state and the control variables associated 
Table 4.1: Wind field for simulation

\begin{tabular}{lc}
\hline \hline Wind along axes & Wind derivative along axes \\
\hline$W_{x}=0$ & $\dot{W}_{x}=0$ \\
$W_{y}=-5.55 \mathrm{~m} / \mathrm{s}$ & $\dot{W}_{y}=0$ \\
$W_{z}=0$ & $\dot{W}_{z}=0$ \\
\hline \hline
\end{tabular}

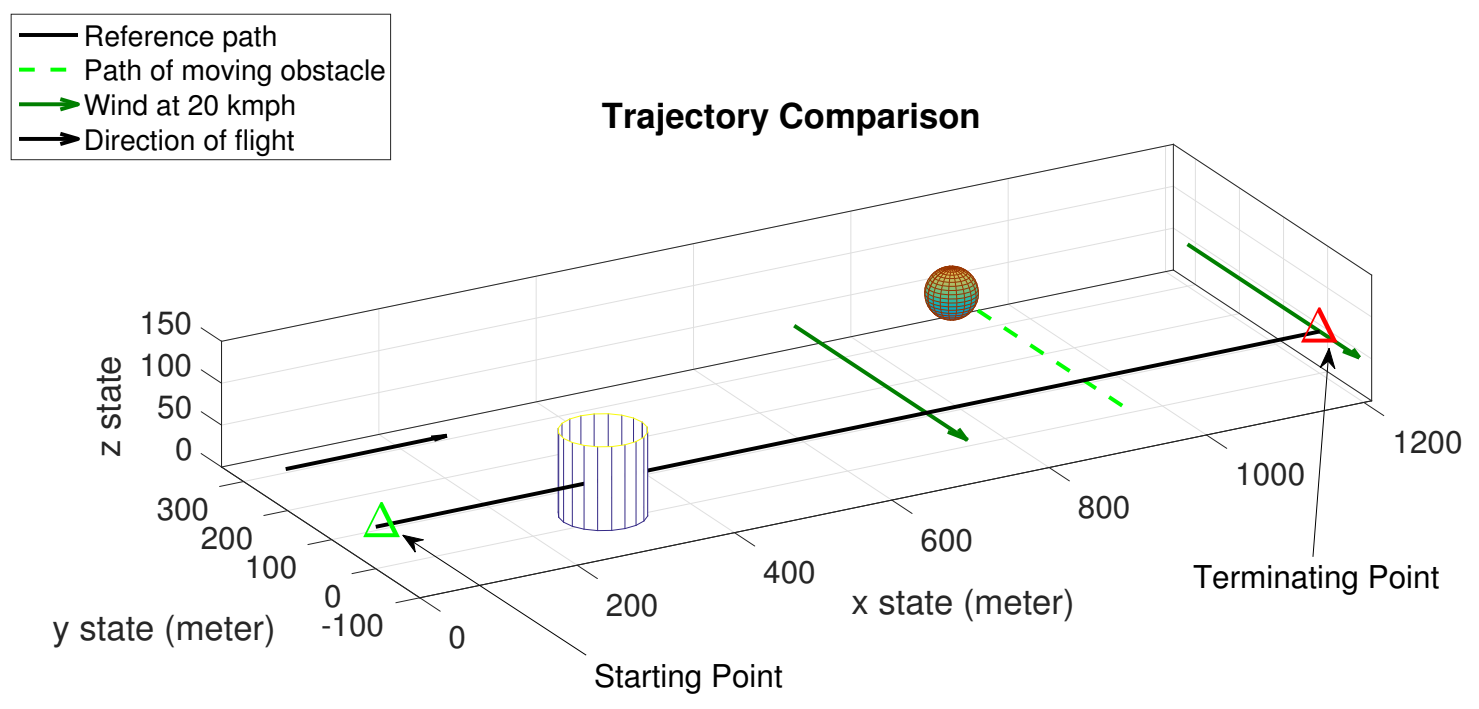

Figure 4.2: Example scenario.

with the dynamic model for the example simulation.

Table 4.2: Bounds on states and control variables for dynamic model.

\begin{tabular}{lcc}
\hline \hline States/Controls & Lower Bound (unit) & Upper Bound (unit) \\
\hline Flight path angle $(\gamma)$ & $-\frac{\pi}{4} \mathrm{rad}$ & $\frac{\pi}{4} \mathrm{rad}$ \\
Yaw angle $(\psi)$ & $-\pi \mathrm{rad}$ & $\pi \mathrm{rad}$ \\
Velocity $(v)$ & $5 \mathrm{~m} / \mathrm{s}$ & $12 \mathrm{~m} / \mathrm{s}$ \\
Angle of attack $(\alpha)$ & $0 \mathrm{rad}$ & $\frac{\pi}{18} \mathrm{rad}$ \\
Roll angle $(\phi)$ & $-\frac{\pi}{4} \mathrm{rad}$ & $\frac{\pi}{4} \mathrm{rad}$ \\
Thrust $(T)$ & $0 \mathrm{~N}$ & $5.4249 \mathrm{~N}$ \\
$\omega_{\alpha}$ (Assume $\left.0.5^{*} \alpha\right)$ & $-\frac{\pi}{36} \mathrm{rad} / \mathrm{s}$ & $\frac{\pi}{36} \mathrm{rad} / \mathrm{s}$ \\
$\omega_{\phi}\left(\right.$ Assume $\left.0.5^{*} \phi\right)$ & $-\frac{\pi}{8} \mathrm{rad} / \mathrm{s}$ & $\frac{\pi}{8} \mathrm{rad} / \mathrm{s}$ \\
$T_{r}\left(\right.$ Assume $\left.0.5^{*} T\right)$ & $-2.71 \mathrm{~N} / \mathrm{s}$ & $2.71 \mathrm{~N} / \mathrm{s}$ \\
\hline \hline
\end{tabular}


The maximum allowable roll angle for the dynamic model is $\phi_{\max }=\frac{\pi}{4}$. Therefore $\bar{n}=1.4142$ for turning flight. Whereas, $\bar{n}=\frac{\bar{L}}{W}=3.0352$ gives the load factor due to maximum lift. Thus with assumption that 1.4142 is the maximum allowable load factor, the minimum pull-up radius, $R_{\min }^{P} \approx 35.44$ meter, and the minimum turning radius, $R_{m i n}^{T} \approx 14.68$ meter. For finding the maximum and the minimum limits of $\omega_{\gamma}$ and $\omega_{\psi}$, the first two inequalities in equation (4.5) are used. Thus the maximum limits on $\omega_{\gamma}$ and $\omega_{\psi}$ can be written as $\frac{\bar{v}}{R_{\min }^{P}}$ and $\frac{\bar{v}}{R_{\min }^{T}}$ respectively, whereas the minimum limits are the negative of the maximum limits respectively. Similarly, the maximum, and the minimum limits on acceleration $a$ are obtained as described in section 4.2.2, where, $\bar{a}$ and $\underline{a}$ are the maximum and the minimum allowable acceleration. Table 4.3 shows the limits on the state and the control variables for the kinematic model. Also, Table 4.4 shows the value of the penalty parameters of the cost function (4.14) for both examples. The penalty parameters $\alpha, \beta$ and $\eta$ are chosen as constant, where $\alpha=\beta=0.5$, and $\eta=0.0001$ and $w$ is chosen according to (4.9) with $q=1, r_{a}=2.25$, and $r_{b}=4.0$ for a stationary obstacle and $r_{b}=8.0$ for moving obstacle. To provide the UAV with more ability to react to moving obstacles compared to stationary obstacles, the value of $r_{b}$ is chosen higher for moving obstacles. A low values is chosen for $\eta$ because the value of the term $\left(x(t)-x_{a}\right)^{2}$ is very large when $x_{a}$ is a distant point and a high penalty associated with that term may affect other objectives. The value of 0.5 for both the $\alpha$ and $\beta$ contribute equally to the cost function for deviation from the reference line along both the axes. Since $w=1$ when a UAV goes near an obstacle the cost due to robustness grows exponentially compared to cost due to other factors $\left(\alpha y^{2}\right.$, and $\left.\beta\left(z-z_{d}\right)^{2}\right)$. Conversely, the $w=0$ when a UAV is far from obstacles, during which the terms with $\alpha, \beta$ and $\eta$ contribute more to the cost function.

Having defined the problem parameters, the original problem of trajectory generation is converted to the discrete model predictive control problem as in (4.17) with the prediction horizon of $N=24$ time-steps at a sampling time of 0.2 seconds, with a control horizon of 1 time-step. Thus, the control update frequency is $5 \mathrm{~Hz}$. After transforming the trajectory generation problem using the dynamic model to the standard quadratic programming (QP) problem as in (2.12), the problem formulation has 7187 non-zero terms in the Karush-Kuhn-Tucker (KKT) matrix. Whereas, the problem with the kinematic model has 7097 non-zero. The number of non-zeros for the kinematic model is slightly lower by approximately $1.3 \%$ compared to the dynamic model, and the number of float- 
Table 4.3: Bounds on states and control variables for kinematic model.

\begin{tabular}{lcc}
\hline \hline States/Controls & Lower Bound (unit) & Upper Bound (unit) \\
\hline Flight path angle $(\gamma)$ & $-\frac{\pi}{4} \mathrm{rad}$ & $\frac{\pi}{4} \mathrm{rad}$ \\
Yaw angle $(\psi)$ & $-\pi \mathrm{rad}$ & $\pi \mathrm{rad}$ \\
Velocity $(v)$ & $5 \mathrm{~m} / \mathrm{s}$ & $12 \mathrm{~m} / \mathrm{s}$ \\
Rate of change of flight path angle $\left(\omega_{\gamma}\right)$ & $-0.3386 \mathrm{rad} / \mathrm{s}$ & $0.3386 \mathrm{rad} / \mathrm{s}$ \\
Rate of change of heading angle $\left(\omega_{\psi}\right)$ & $-0.8175 \mathrm{rad} / \mathrm{s}$ & $0.8175 \mathrm{rad} / \mathrm{s}$ \\
Acceleration $(a)$ & $0 \mathrm{~m} / \mathrm{s}^{2}$ & $9.81 \mathrm{~m} / \mathrm{s}^{2}$ \\
$\omega_{\gamma_{r}}\left(\right.$ Assume $\left.0.5^{*} \omega_{\gamma}\right)$ & $-0.1693 \mathrm{rad} / \mathrm{s}^{2}$ & $0.1693 \mathrm{rad} / \mathrm{s}^{2}$ \\
$\omega_{\psi_{r}}$ (Assume $\left.0.5^{*} \omega_{\psi}\right)$ & $-0.4088 \mathrm{rad} / \mathrm{s}^{2}$ & $0.4088 \mathrm{rad} / \mathrm{s}^{2}$ \\
$a_{r}\left(\right.$ Assume $\left.0.5^{*} a\right)$ & $-4.905 \mathrm{~m} / \mathrm{s}^{3}$ & $4.905 \mathrm{~m} / \mathrm{s}^{3}$ \\
\hline \hline
\end{tabular}

Table 4.4: Penalty parameters to the cost function (4.14)

\begin{tabular}{lc}
\hline \hline$\alpha=0.5$ & $\beta=0.5$ \\
$\eta=1 e-4$ & $w$ as in $(4.9)$ where $q=1$ \\
& $r_{a}=2.25$ as in $(4.11)$ \\
& $r_{b}=4.0$ for stationary obstacle, \\
& and $r_{b}=8.0$ for moving obstacle as in $(4.11)$ \\
\hline \hline
\end{tabular}

ing point operations required is approximately similar ([62] Appendix C.1). Therefore, both the problem formulations have approximately similar computational complexity.

Now, the trajectory generated between the kinematic and the dynamic problem formulations are discussed. Figures 4.3 and 4.4 show the resulting flight trajectories. It can be seen that in both the formulations they stay close to the reference path while avoiding the obstacles. Figure 4.4 is the top view of Figure 4.3. In the figures a black color arrow shows the direction of flight. Figures 4.5 and 4.6 show close up views of the trajectories as they encounter the stationary obstacle, and the moving obstacle and pass through the wind field respectively. In Figures $4.5 \mathrm{a}$ and $4.5 \mathrm{~b}$ the trajectory generated using the kinematic model deviates more than the trajectory generated using the dynamic model despite the kinematic model having the turning and the pull-up radii lower than the obstacle radius. This is mainly due to the choice of the length of finite horizon. However, even in such case the dynamic model performs better. A longer finite horizon for the kinematic model may result in a better trajectory, but with longer the prediction horizon (finite horizon) for a fixed sampling time, the problem becomes larger 
and more computationally intensive, and thus is undesirable. In Figure 4.6a at the point, $x=700$ meter it can be observed that the trajectory are disturbed which is due to the encounter of wind velocity of $20 \mathrm{~km} / \mathrm{h}$. The trajectory generated using the nonlinear model predictive control (MPC) cannot completely compensate for the disturbance due to the constant wind; however, in comparison, the dynamic model is less affected by the disturbance than the kinematic counterpart (see Figure 4.6a). Moreover, from Figure 4.6 , it can be seen that the trajectory using the kinematic model deviates more from the reference path compared to the trajectory using the dynamic model during obstacle avoidance.

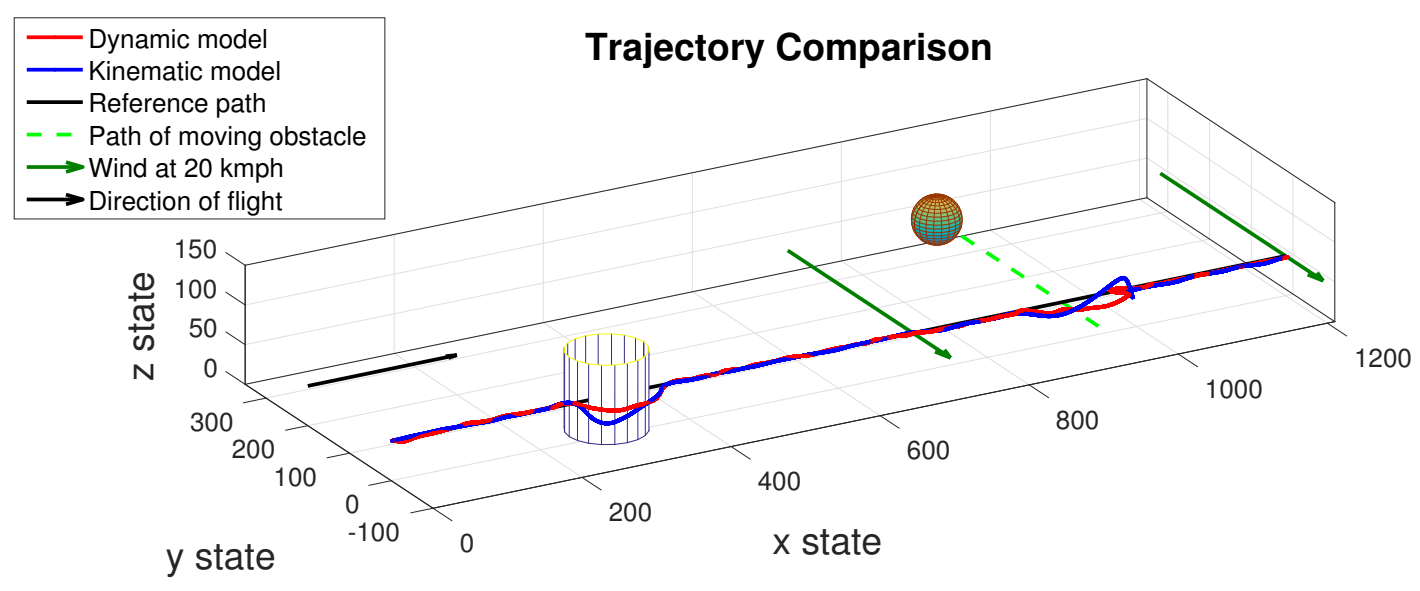

Figure 4.3: Flight trajectory of the Fixed-Wing UAV, 3D view

\section{Trajectory Comparison}

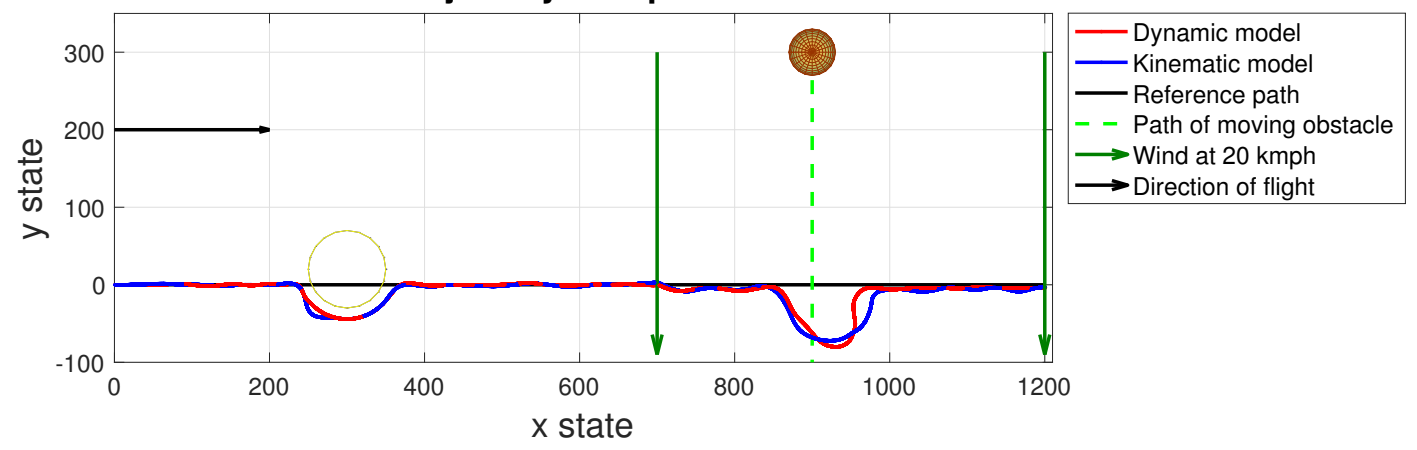

Figure 4.4: Flight trajectory of the Fixed-Wing UAV, XY view

In order to compare the trajectory in terms of deviation from the reference path, the 


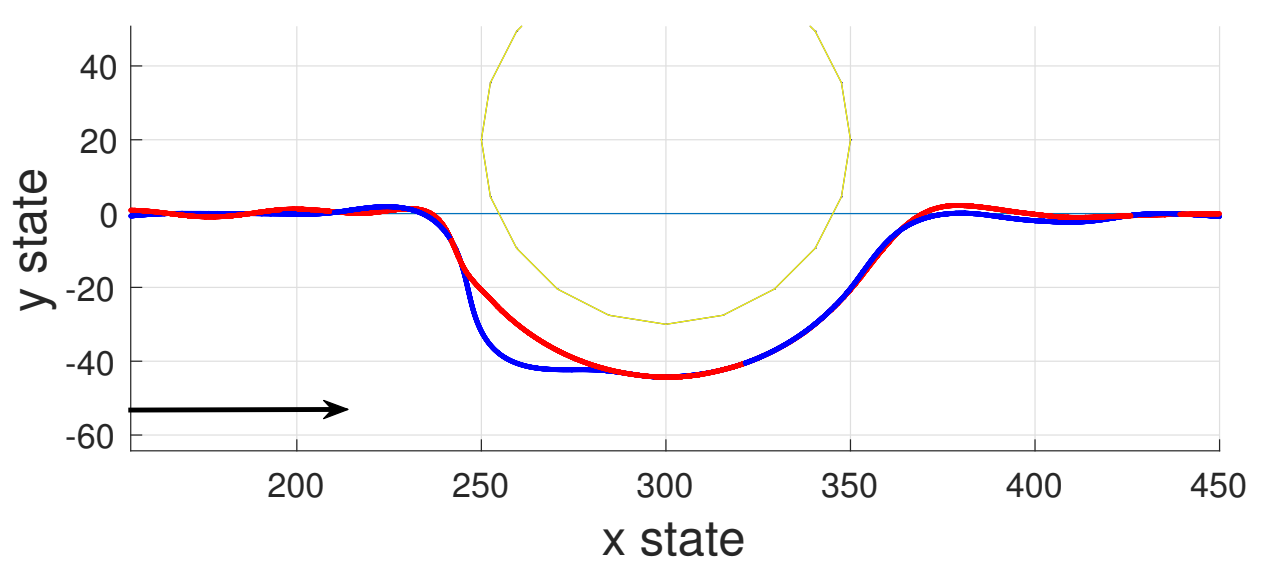

(a) $\mathrm{XY}$ view.

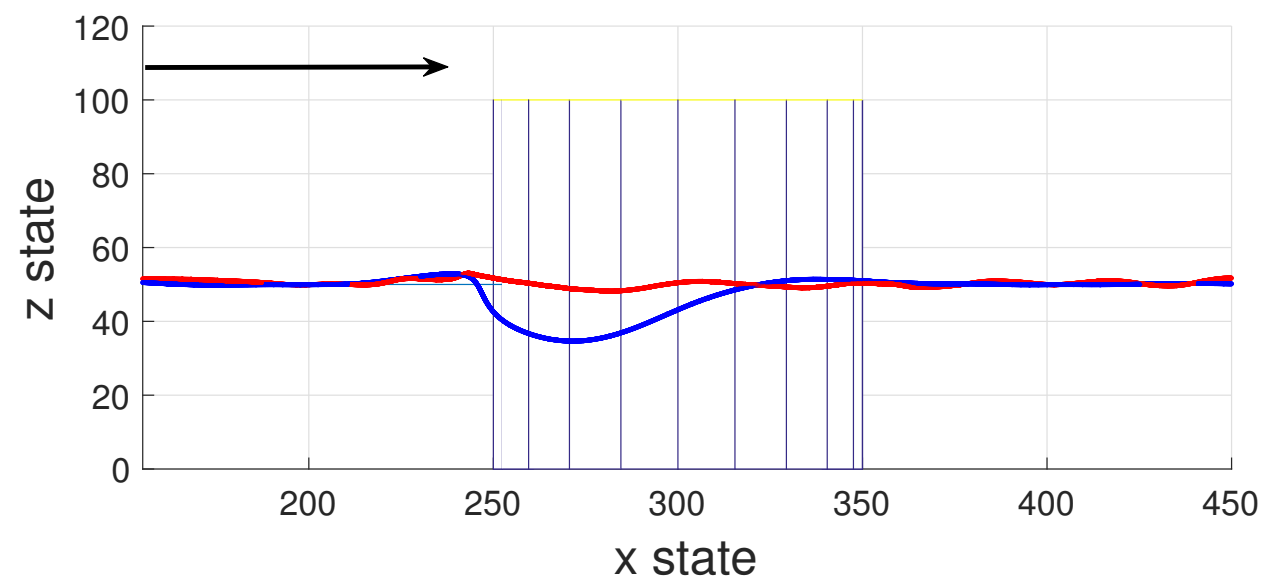

(b) XZ view.

Figure 4.5: Flight trajectory of the Fixed-Wing UAV; zoomed near the stationary obstacle.

trajectory is denoted by 1200 points approximately equispaced (along the trajectory), and sum the deviation of each point from the reference path and normalize the sum by dividing it with 100 to signify it as the total cost of the flight trajectory. From the calculation, the cost are obtained as 193.2 units and 223.9 units using the dynamic and 


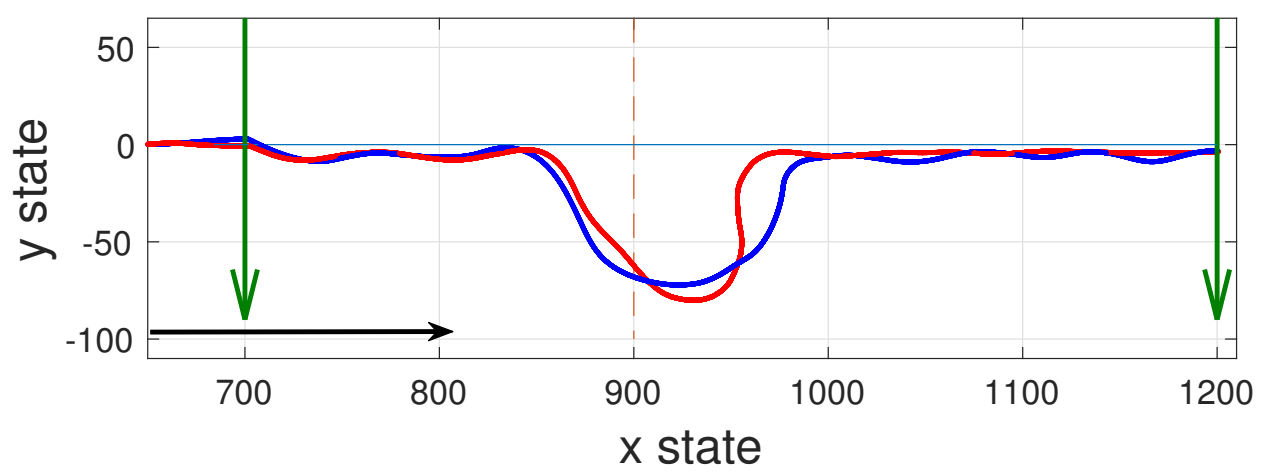

(a) XY view

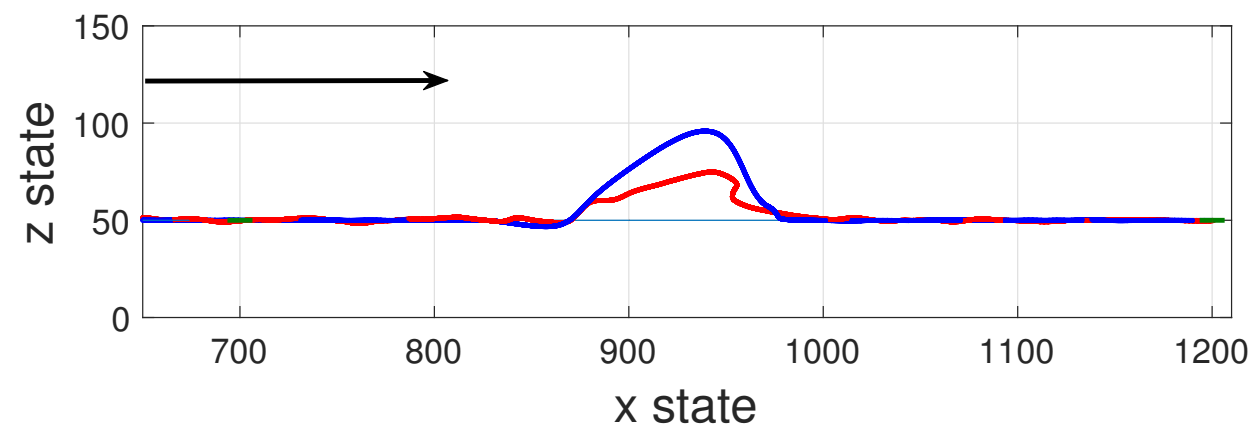

(b) $\mathrm{XZ}$ view

Figure 4.6: Flight trajectory of the Fixed-Wing UAV; zoomed at the constant wind region.

the kinematic model respectively.

Figure 4.7 shows the computation time per model predictive control (MPC) update, where the legend of the figure shows the time to solve the discrete optimal control problem at a given instant for the kinematic and the dynamic based formulation respectively. For the example simulation, a laptop (Macbook Air 2012) with a $1.8 \mathrm{GHz}$ processor and $4 G B$ of $R A M$ is used unless otherwise stated. In the figure, it can be seen that the computation time taken to solve (4.16) at a given time instance is within 4.82 to 12.81 
milliseconds using the custom solver in Matlab environment whereas the sample period is 200 milliseconds. Therefore, the actual computation time is well suited for real-time implementation for the given problem. Although the maximum solution time is important in terms of real-time implementation which is 12.81 milliseconds for the problem using the dynamic model and 12.73 milliseconds for the problem using the kinematic model, in terms of average computation time it is 6.76 and 6.35 milliseconds for the problem using the dynamic and the kinematic model respectively. For the example case, the flight time to reach the point $x=1200$ meters along the reference path using the dynamic model is slightly more than its kinematic counterpart. It takes 125.8 seconds for the dynamic model and 120.0 seconds for the kinematic model to complete the flight to the same point along the desired reference path.

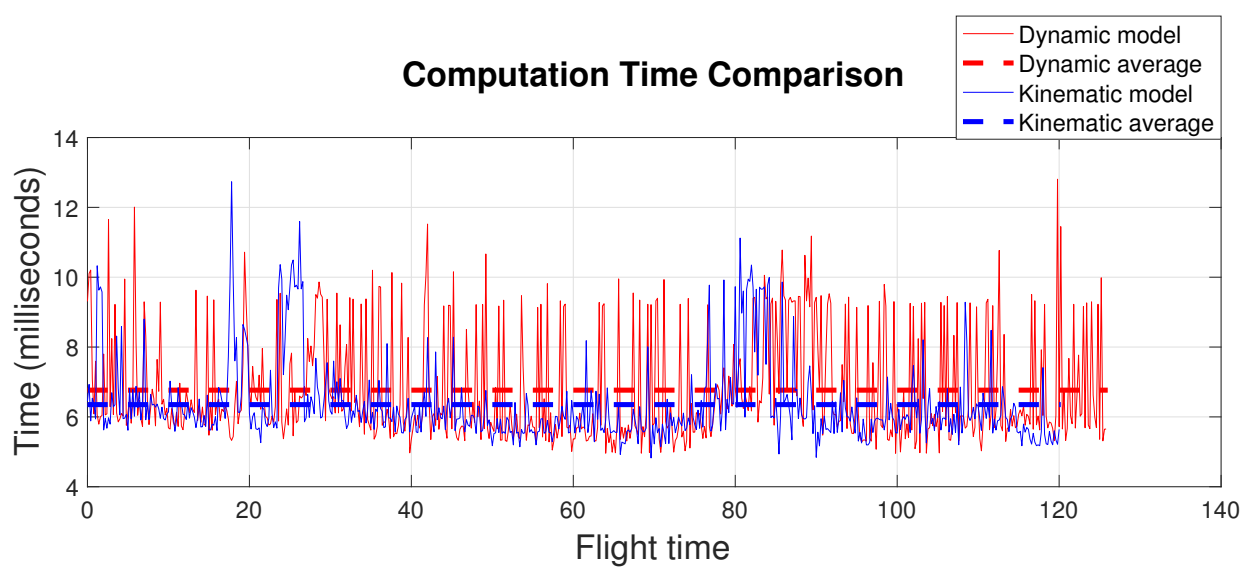

Figure 4.7: Computation time per instance (0.2 second) for online simulation using an Intel processor.

Next, the computation times on a less capable processor is examined, such as would be available on a UAV. Figure 4.8 shows the computation time when the problem are solved on a Raspberry PI 3B computer [95]. Raspberry PI 3B is an ARM (Advanced RISC Machines, RISC: reduced instruction set computer)-based platform with $1.2 \mathrm{GHz} \mathrm{CPU}$ and $1 G B$ of RAM. Although there are other ARM-based platform which are more capable then Raspberry PI 3B, the aim here is to present that a low solution time is possible in such a platform using the approach that utilizes custom solver. In our experience, the computation time in Raspberry PI 3B is highly affected by factors such as programs running in the background, temperature of the CPU and even a slight cursor (mouse) 
movement. The computation time to solve (4.16) at a given time instance using a custom solver in the computer is within 71.24 to 192.1 milliseconds. In the figure, an average computation time per instance is shown by dashed lines for both the problems using the dynamic and the kinematic model and it is 152.1 and 120.5 milliseconds respectively. Also, the computer may not always produce such a low solution time if there are more obstacles to be considered.

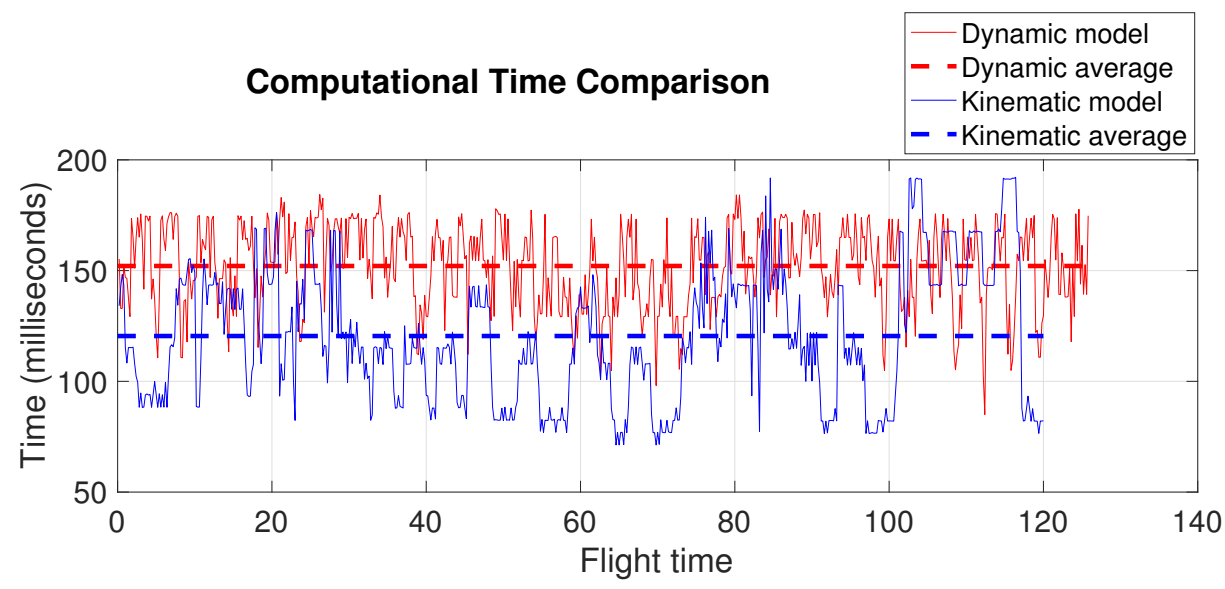

Figure 4.8: Computation time per instance for online simulation using an $A R M$ processor.

While customization of a quadratic programming (QP) solver aims to use it in an embedded platform, it also eliminates the unnecessary computational overhead used in platforms such as Matlab. A comparison between the dynamic and the kinematic model for trajectory generation shows that the trajectory generated by the dynamic model can stay closer to the desired or the reference path while avoiding obstacles in the presence of the disturbance compared to its kinematic counterpart while taking similar computation time per instances. Hence, it can be said that the customization of the solver to solve a standard quadratic programming (QP) problem and the problem formulation adopted, jointly have real-time implementation usage.

\section{Comparison with ECOS and IPOPT}

In the following, the custom solver is compared with ECOS and IPOPT for solving the problem scenario in this Section 4.4.1. For the fair comparison, the custom solver 
is replaced with ECOS and IPOPT for solving the above example scenario within the program file. All the problem defining parameters for this comparison are exactly the same as for the above scenario except for the value for $\eta$. Since with $\eta=1 e-4$ the trajectory generated with IPOPT could not reach the terminating point, therefore, $\eta$ is set to 0.005 for all three solvers. In the following cost and computation time are compared.

Table 4.5 shows the comparison between IPOPT, ECOS and the custom solver (referred as CS) for the scenario using the Kinematic model for fixed-wing UAV. With regard to the value of cost the trajectory using IPOPT and CS are closer compared to with ECOS. However, in terms of average computation time IPOPT is more than ten times more computationally intensive while ECOS is more than twice slower than CS.

Table 4.5: Comparison of the custom solver (CS) with IPOPT and ECOS: For trajectory with Kinematic model.

\begin{tabular}{cccc}
\hline \hline & Cost & Average computation time $(\mathrm{ms})$ & Maximum computation time (ms) \\
\hline IPOPT & 185.8 & 215.1 & 991.1 \\
\hline ECOS & 205.3 & 24.8 & 62.1 \\
\hline CS & 187.1 & 7.2 & 13.4 \\
\hline \hline
\end{tabular}

Similarly, Table 4.6 shows the comparison between the solvers for the scenario using the Dynamic model for fixed-wing UAV. In terms of the value of cost, IPOPT performs better than CS. In among the trajectories with the solvers, ECOS deviates the most for the case of Dynamic model. In terms of computation time, CS solves the problem with the maximum computation time of 12.1 milliseconds, while IPOPT is slowest among them with 870.7 milliseconds, and ECOS is at least four times slower than CS.

Table 4.6: Comparison of the custom solver (CS) with IPOPT and ECOS: For trajectory with Dynamic model

\begin{tabular}{cccc}
\hline \hline & Cost & Average computation time (ms) & Max computation time (ms) \\
\hline IPOPT & 166.0 & 134.9 & 870.7 \\
\hline ECOS & 178.5 & 21.7 & 56.8 \\
\hline CS & 172.5 & 6.8 & 12.1 \\
\hline \hline
\end{tabular}




\subsubsection{Study on the Choice of Time Step, $\Delta t$ for Trajectory Gen- eration}

The time step $\Delta t$ is used to approximate the system dynamics or kinematics on a discretized time-scale (with fixed-time intervals). A linear approximation to the system dynamics or kinematics at fixed-time intervals are made. The choice of $\Delta t$ determines the accuracy of system approximation [117]. While discretizing system dynamics or kinematics, its controls are discretized at the time step $\Delta t$ as well; therefore, it also determines the controls resolution. Moreover, for a finite horizon problem with fixed final time, the choice of time step determines the problem size. The aim of this trajectory generation is a real-time application, for which the problem needs a regular update from the environment (update on obstacle locations). The trajectory generation used is a high-level controller that generates an avoidance trajectory. Therefore, the rate at which environmental information has to be updated to the problem can also be a determining factor for the choice of $\Delta t$. Thus, a tradeoff between the environmental update rate, the length of the finite horizon and problem size to be solved in a particular hardware system collectively can determine the choice of $\Delta t$ for this type of problem. The increase in the length of finite horizon $H_{p}$ with a given $\Delta t$ increases the problem size, or with the decrease in time step $\Delta t$ for a given $H_{p}$, the problem size still increases. Moreover, a coarse approximation of the system equation is obtained by the increase in the value of $\Delta t$. Figure 4.9 and 4.10 present a brief numerical study on trajectories generated with the change in $\Delta t$ using the kinematic and the dynamic models respectively for a fixed $H_{p}$.

Figure 4.9 presents trajectories using the kinematic model with $\Delta t=0.5,1,1.5,2$ seconds for the fixed $H_{p}=6$ seconds. In this study for varying $\Delta t$, a single stationary obstacle is considered for simplicity. Also, the length of time step $\Delta t$ is chosen higher (compared to the example in 4.4.1) in order to analyze the trajectory with a higher sampling time, as higher the $\Delta t$ the lower is the number of steps $(N)$ for the fixed prediction horizon. To minimize the update rate of the model predictive control (MPC), it is clearly beneficial to find the largest possible Delta t, still providing a good trajectory. The effect of $\Delta t$ on the trajectory can be observed from Figures 4.9 and 4.10 and also from the example in 4.4.1. Since only a single obstacle is considered in this section, an observation can be made at the first part of the trajectory in section 4.4.1, and this is 
reasonable, since the trajectory is not affected by the second obstacle or the wind field until the UAV is well past the first obstacle. The robustness function guarantees that the second obstacle does not affect the trajectory generated passing around the first obstacle. It can be seen in both the figures 4.9 and 4.10 that with a high $\Delta t=2000$ and $\Delta t=1500$ milliseconds, the trajectory generated is unsafe for a UAV to avoid a stationary obstacle, and from the example scenario considered in section 4.4.1, with $\Delta t=200$ milliseconds the UAV gets to avoid even a moving obstacle. All the parameters (UAV parameters and penalty parameters) used in this subsection are exactly the same to the case of section 4.4.1. Brief on results from this subsection are discussed below.

Figure 4.9 presents two subfigures, the XY and XZ views of the trajectories. In the figures the trajectories due to the problem with the kinematic system model are presented with varying $\Delta t$. The $\mathrm{XY}$ view of the plot shows a clear distinction between the nature of the trajectories generated with each $\Delta t$ chosen. The trajectories generated using $\Delta t=1000,1500$, and 2000 milliseconds deviate from the reference path almost from the beginning, where the trajectory with $\Delta t=1000$ milliseconds makes safe maneuver around the obstacle, however the trajectory does not remain as close to the reference path (see figure $4.9 \mathrm{~b}$ ) as the trajectory with $\Delta t=500$ milliseconds. Thus, in among the trajectories, the one with $\Delta t=500$ milliseconds can safely maneuver around the obstacle and after the avoidance it remains close to the reference flight path. In the figure, the trajectory with $\Delta t=2000$ milliseconds is considered as failed since it will hit the obstacle if allowed to further continue in simulation. While the trajectory with $\Delta t=1500$ milliseconds is unacceptable since it returns back towards the initial point and does not progress along the desired direction of flight (refer to subfigure 4.9a).

Similarly, figure 4.10 presents trajectories for the dynamic model with $\Delta t=500,1000$, 1500 and 2000 milliseconds. In the figure, two sub-figures present XY and XZ views of the trajectories. For $\Delta t=1000,1500$ and 2000 milliseconds, the trajectory generation problem using the dynamic model does not produce flyable trajectory for a UAV. For the case of $\Delta t=1000$, and 2000 milliseconds, the trajectories become unacceptable as they do not progress along the desired direction of flight, while the trajectory with $\Delta t=1500$ milliseconds fails since it will hit the obstacle if further allowed to continue in simulation (see figure 4.10a). For the problem with the dynamic model, $\Delta t=500$ milliseconds gives the finest trajectory among them.

In Figures 4.9 and 4.10 the lower the $\Delta t$, the trajectory is better. However, the 


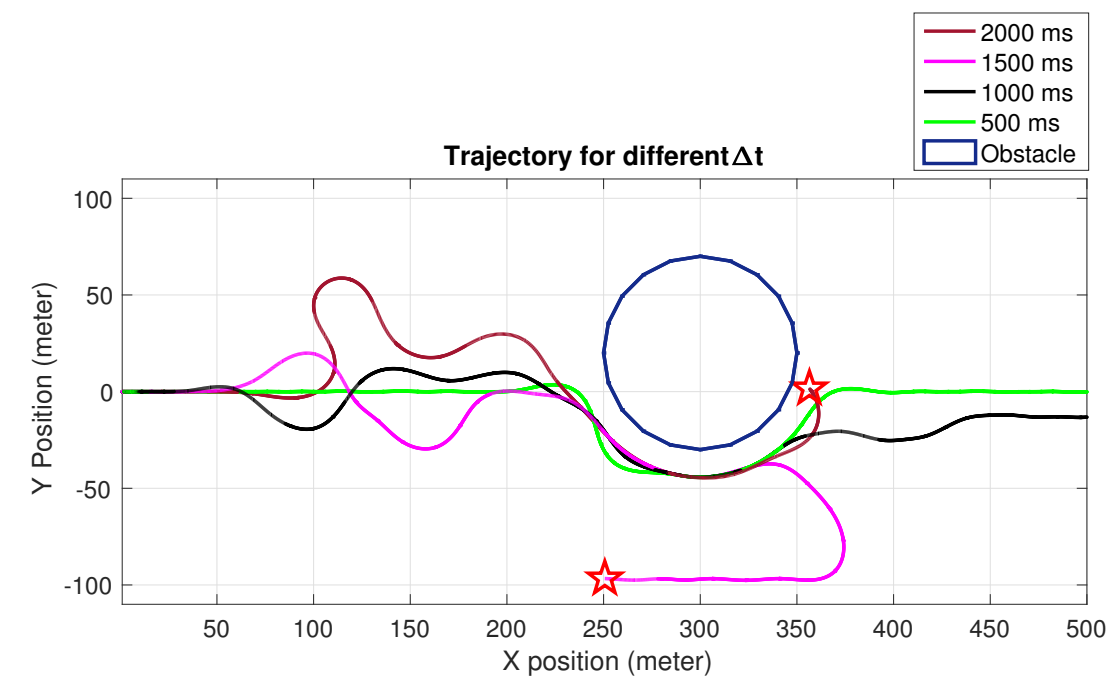

(a) XY view

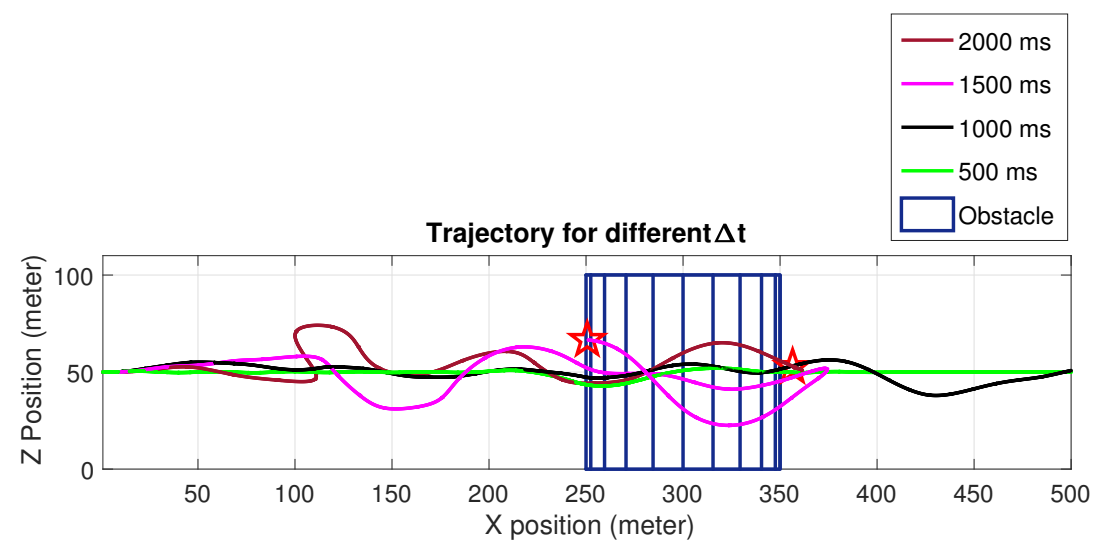

(b) XZ view

Figure 4.9: Trajectories using the kinematic model for different value of $\Delta t$ 


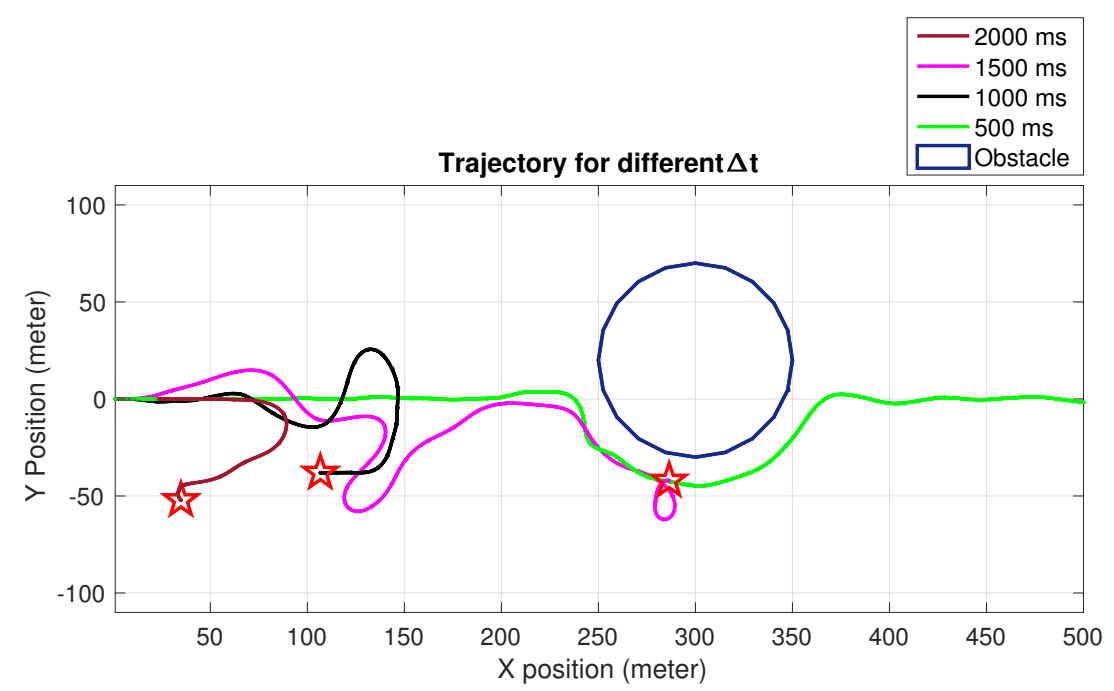

(a) XY view

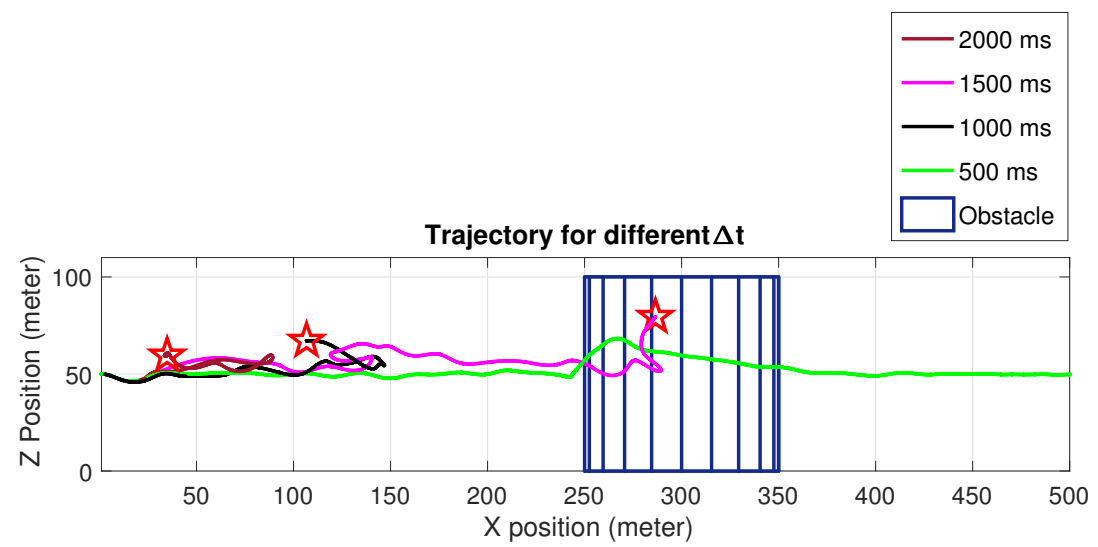

(b) XZ view

Figure 4.10: Trajectories using the dynamic model for different value of $\Delta t$ 
problem size increases with the decrease in $\Delta t$ for a fixed $H_{p}$, and with the increase in problem size the computation time increases. Therefore, the final choice for $\Delta t$ will need to be determined by the designer specifically for a given UAV and the expected operational environment. This may be done by numerical tuning with a high fidelity simulator.

\subsubsection{Monte-Carlo Test}

Apart from the numerical examples in sections 4.4.1 and 4.4.2, a Monte-Carlo test is performed, where the problem are initialized (using the kinematic and the dynamic model) with random nominal state-control pairs and random obstacle parameters (that defines its location, shape, and size using (4.7)). For the test, the number of obstacles ranges from 2 to 20, the obstacle sizes (which depends on $a_{i}, b_{i}$, and $c_{i}$ in (4.7)) range from 20 to 50 meter, the shapes of the obstacles range (determined by $p_{x}, p_{y}$, and $p_{z}$ in (4.7)) from 2 to 10, and the locations of the obstacles in 3D space as defined below are all uniformly distributed. Obstacles for the test are defined such that they are distributed over the space $100 \sim 1200$ meter along the positive $x$ - axis, $-400 \sim 400$ meter along the y-axis and $0 \sim 300$ meter along the z-axis. Such a distribution area is chosen so that the obstacles may fall in or along the desired reference path and as a consequence, it may be possible to observe an avoidance maneuver taken by a UAV. Obstacles in the space are initialized such that they range from $0 \sim 1200$ meter along the $x$-axis (however, obstacles will be initialized between $100 \sim 1200$ meter to avoid obstacles near the initial point), $-400 \sim 400$ meter along the $y$ - axis, and $0 \sim 300$ meter along the $z$ - axis. In any problem initialized there can be both stationary and moving obstacles. Moving obstacles are given velocity $V_{O}$, and angles $\psi_{O}$ and $\gamma_{O}$ are two angles about the positive $x-$ axis and above the $x-y$ plane respectively. These moving obstacle parameters are drawn from ranges $V_{O} \in[0.5,5.55] \mathrm{m} / \mathrm{s}, \psi_{O} \in[-\pi, \pi] \mathrm{rad}$, and $\psi_{O} \in[-\pi / 2, \pi / 2] \mathrm{rad}$. The velocity for moving obstacles are chosen such that it is lower than the UAV's minimum velocity. Thus, during the simulation, the position of a moving obstacle is obtained as follows, 


$$
\begin{gathered}
\dot{x}_{O}=V_{O} \cos \left(\psi_{O}\right) \cos \left(\gamma_{O}\right) \\
\dot{y}_{O}=V_{O} \sin \left(\psi_{O}\right) \cos \left(\gamma_{O}\right) \\
\dot{z}_{O}=V_{O} \sin \left(\gamma_{O}\right)
\end{gathered}
$$

For each scenario the number of obstacles are chosen with uniform distribution. There is at least one stationary and one moving obstacle, and at the maximum, there are ten stationary and ten moving obstacles. In addition, there is (constant) wind conditions (for each initialization which forms a scenario) where value of the wind velocity is taken by uniformly distribution over $0.5 \sim 5.55 \mathrm{~m} / \mathrm{s}$, in between $x=700 \sim 1200$ meters similar to Figure 4.3. Thus, with the same problem defining parameters (finite horizon, time step, UAV parameters, and penalty parameters (except $\eta$ which has been set to $\eta=0.01)$ ) used for the example in 4.4.1 100 random tests are performed. As stated in the introduction and in the problem formulation sections of this chapter, a goal point is not considered. However, in order to have a meaningful way to terminate the simulation and compare results between the kinematic and dynamic trajectories a point is assigned along the desired reference path where the simulation is stopped. As subsection 4.4.1, the simulation is stopped once a UAV reaches or crosses $x=1200 \mathrm{~m}$. The following presents a summary of the results.

The UAV parameters and constraints used in the example 4.4.1 for the case of dynamic and kinematic models are used for the simulation as well. The UAV starts from the point $x=0, y=0 \mathrm{~m}$ at the height of $z=50 \mathrm{~m}$ and flies towards the desired direction. Figure 4.11 presents the total flight time per scenario in seconds, which shows that, it took $100 \sim 120$ seconds of flight time for $98 \%$ of scenarios for a UAV using the dynamic model, while it took the same flight time for $89 \%$ of scenarios using the kinematic model. Using the dynamic model only $1 \%$ of scenario took $120 \sim 140$ seconds of flight time, while using the kinematic model $10 \%$ of scenario took the same time. In all the scenarios the UAV reaches the terminating condition. It is always better to have a low flight time as it signifies that a UAV stayed closer to the reference path. Since the reference path is the shortest distance between the origin and the assumed goal point towards the direction of flight, the deviation from the path may increase the flight time.

Figure 4.12 shows the computation time taken by the custom solver to solve for the model predictive control (MPC) problem at that time instant (at a given time $t$ before moving to the next model predictive control (MPC) window) throughout all the 


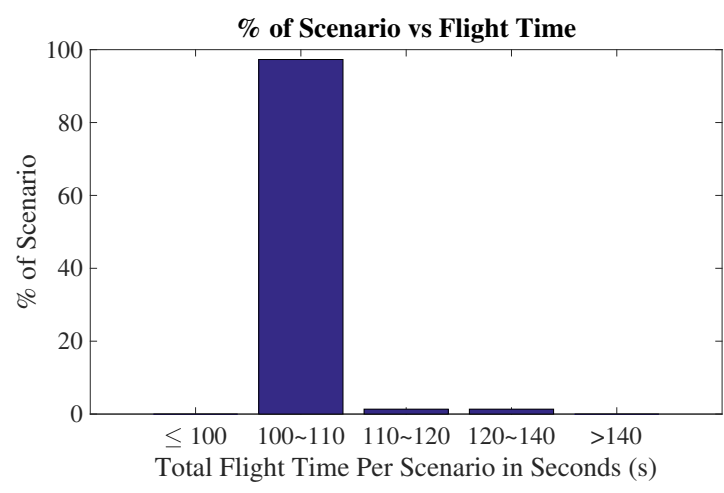

(a) The case of dynamic model

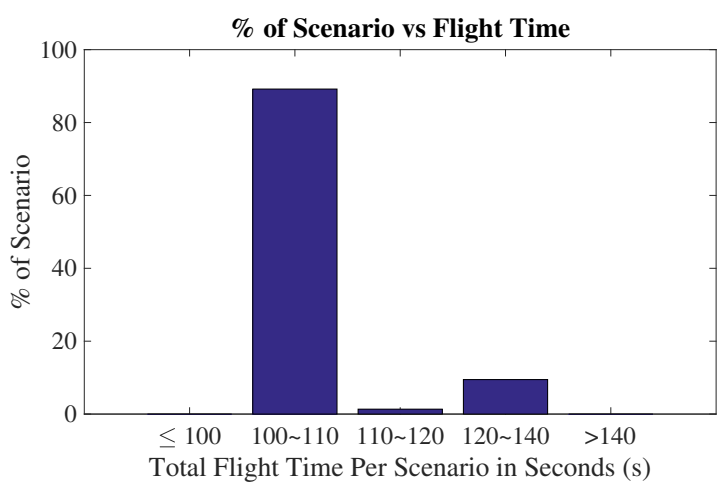

(b) The case of kinematic model

Figure 4.11: The total flight time per scenario

scenarios. It can be seen that for $100 \%$ of the instances it takes less then or equal to 15 milliseconds of computation time per model predictive control (MPC) update using the dynamic model of the UAV, with approximately $95 \%$ of them taking less than 10 milliseconds, whereas for the case of the kinematic model more than $76 \%$ of them take more than 10 milliseconds of the solution time. The solution time of $15 \sim 20$ milliseconds for about $3 \%$ of instances using the kinematic model signals that the problem with the dynamic model is relatively faster then the kinematic counterpart (using Macbook Air laptop with a $1.8 \mathrm{GHz}$ processor and $4 \mathrm{~GB}$ of $\mathrm{RAM}$ )

In order to measure the deviation of trajectories from the reference path, the expression in (4.20) is used, which finds the total value of the square of deviation for each Scenario at states history traced out by a UAV from the starting point.

$$
\text { Square of Deviation }\left(D_{s}\right)=\sum_{i}\left[\alpha y_{i}^{2}+\beta\left(z_{i}-z_{d}\right)^{2}\right]
$$

with $\alpha=1, \beta=1$ and $i$ is the number of state history traced out by a UAV during each scenario.

Figure 4.13 shows the value of the square of deviation. Figure 4.13a shows that more than $88 \%$ of the scenarios tend to remain close to the reference path with the square of deviation less than or equal to $2 \times 10^{5}$ using the dynamic model while $6 \%$ of scenarios for the case of kinematic model take the similar deviation value as seen in Figure 4.13b. 


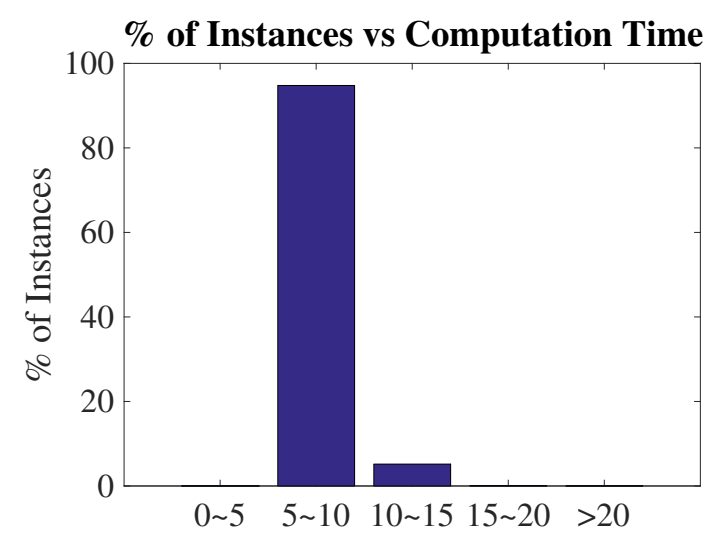

Computation Time Per Instances in milliseconds

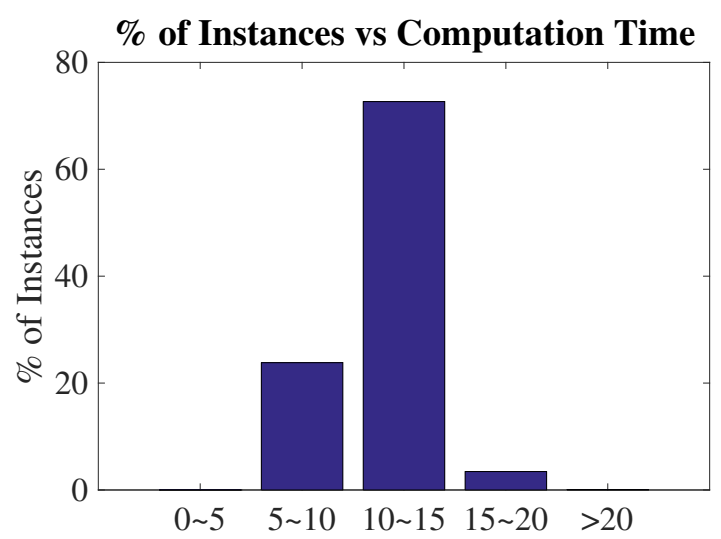

Computation Time Per Instances in milliseconds

(a) The case of dynamic model

(b) The case of kinematic model

Figure 4.12: Computation time per instant for all scenarios

There are $8 \%$ of scenarios (in the kinematic model) where a UAV finds a way to avoid obstacles ignoring the desire to remain with the reference path, in such cases the value of the deviation are much higher $\left(>8 \times 10^{5}\right)$ which is shown in figure $4.13 \mathrm{~b}$.

\subsection{Summary}

The chapter presented an approach for real-time generation of trajectory and autonomous obstacle avoidance for a fixed-wing UAV using the dynamic model with a custom solver, and comparison with its kinematic counterpart. The Monte-Carlo results show, the dynamic model performs better in terms of maneuverability, computation time and the total flight time compared to with the kinematic model. In addition, the trajectory generated using the dynamic model deviates less from the desired reference path compared to the kinematic model even in the presence of the wind disturbance, making the trajectory generation more robust compared to with kinematic model. Although the use of a non-linear model of a fixed-wing UAV for autonomous obstacle avoidance is thought to be computationally intensive, the issue has been tackled by customization of a quadratic programming (QP) solver at the code level. Moreover, from the availability of low-cost computing platforms like Raspberry PI 3B and many other credit-card sized computers with processing capability higher then the one used in the simulation, it is safe to say 


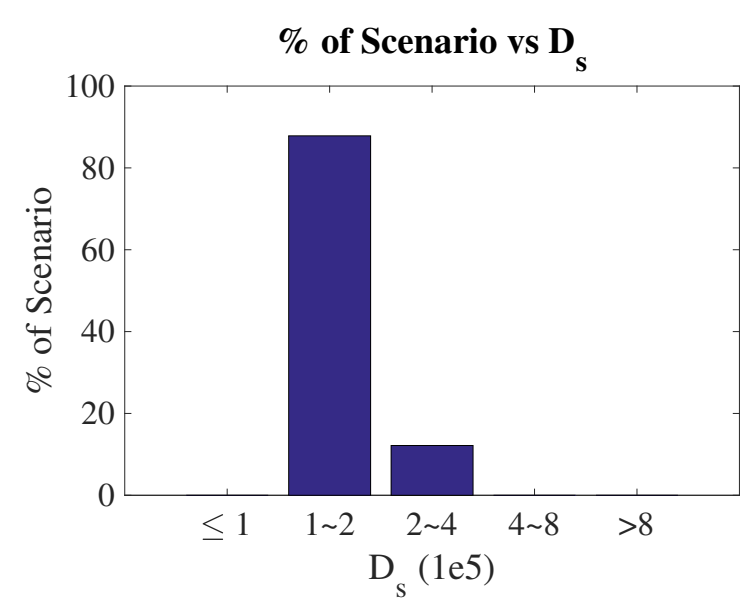

(a) The case of dynamic model

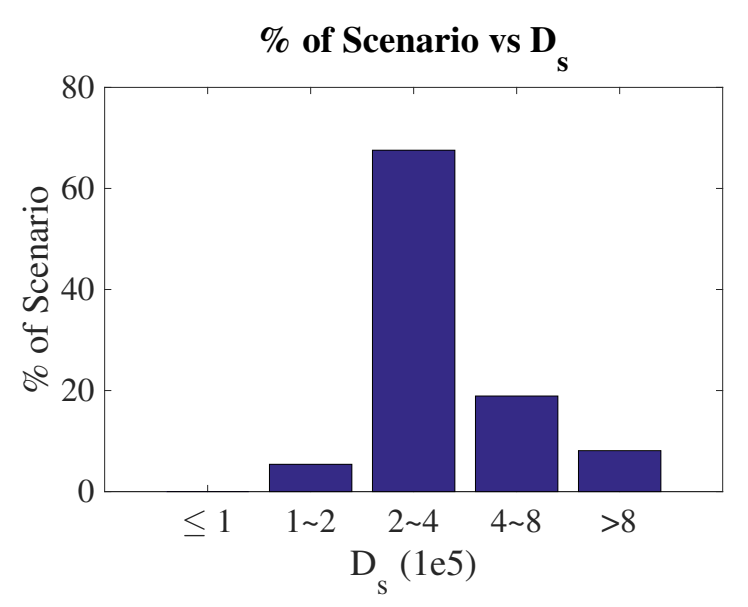

(b) The case of kinematic model

Figure 4.13: Total value of square of devation

that the method shown in this chapter is applicable for rapid generation of trajectory and autonomous obstacle avoidance. Finally, the Monte-Carlo test with the dynamic and the kinematic model presents the usability of the method. 


\section{Chapter 5}

\section{Real-Time Trajectory Generation for Quad-rotor UAVs}

The problem of trajectory generation and autonomous obstacle avoidance for fixed-wing UAVs was examined in Chapter 4 using the model predictive control (MPC) framework. Whereas, in this chapter, the method is adapted to the case of quad-rotor UAVs. Even though quad-rotors have limits on turning and pull-up rates constrained by the maximum power available, in contrast to the case of fixed-wing UAVs, they can stop mid air and perform maneuver around obstacles. Therefore, the rapid solvability of the problem for quad-rotor may not be as critical as for the fixed-wing UAVs. However, quad-rotors can have advantages in a fast and cluttered environment with a low solution time. Next, a dynamic model for quad-rotors is often used for the study of controls, however, for trajectory generation a kinematic model may be sufficient. Therefore, problems using a dynamic and a kinematic model for quad-rotor UAVs are formulated, and an approach to retrieve controls for the dynamic model using the control solution of the kinematic model is summarized. A comparative study between the problems using the dynamic and the kinematic model in a credit-card sized computer; Raspberry PI 3B, shows that the kinematic model is at least twice faster than the dynamic model. Also, in approximately $88 \%$ of the instances in the Monte-Carlo test the trajectories between these models have a similar value of the cost function. 


\section{$5.1 \quad$ Introduction}

Although the quad-rotors have the benefits of being able to hover at a point in contrast to the fixed-wing UAVs, the low solution time for a quad-rotor trajectory generation is beneficial when rapid obstacle avoidance is necessary. Moreover, the necessity for low computation time increases when quad-rotors are in a swarm; due to the increased complexity including collision avoidance among quad-rotors. Therefore, rapid trajectory generation for a swarm of quad-rotor UAVs with obstacle and collision avoidance is the topic of ongoing research and it gets complex with an increased number of swarm agents. Thus, to understand the computability of a swarm problem, it is necessary to study the complexity of rapid trajectory generation associated with a quad-rotor UAV. Therefore, as similar to the comparative study for a fixed-wing UAV in Chapter 4, the comparison between a dynamic and a kinematic model for quad-rotor UAVs are studied so that the study can pave a way for a swarm application, which will be dealt with in Chapter 6 of this thesis. In order to do so, a dynamic and a kinematic model for a quad-rotor are defined, and then an approach is shown where solutions to the kinematic model are used to retrieve the actual control parameters for the dynamic model. Although a slight variation of the approach also appears in [118] where the author used it for calculation of command inputs for trajectory tracking, the derivation shown in this thesis is in reduced form tailored for the nature of our problem. Since, our concern is on trajectory generation and not on replacing an on-board low level flight controller, the purpose of control calculation is to realize a trajectory during simulation by integrating controls into a system model. Thus, with an approximate computational complexity associated with a kinematic model controls for a quad-rotor dynamic model could be obtained. As similar to the case of a fixed-wing UAV, a control problem is defined, which is then solved by the method of finite horizon model predictive control as outlined in Section 4.3. The custom solver from Chapter 2 is then applied to obtain rapid solution times. The Monte-Carlo study shows that the kinematic model performs computationally better compared to the dynamic model, while the dynamic model slightly outperforms the kinematic model in fulfilling the performance index of the control problem.

This chapter is organized as follows. Section 5.2 presents problem formulation using two different models for a quad-rotor. Section 5.3 presents numerical examples. In the numerical example section, the effect of sample time on the quad-rotor models is studied 
by solving the problem under consideration. Trajectory generation using the dynamic and kinematic models are compared, and a Monte-Carlo study is presented. Finally, Section 5.4 summarizes the chapter.

\subsection{Problem Formulation}

\subsubsection{Quad-rotor System Equations}

\section{A Dynamic Model}

Let us consider a dynamic model for a quad-rotor UAV used for simulations in [119], given by

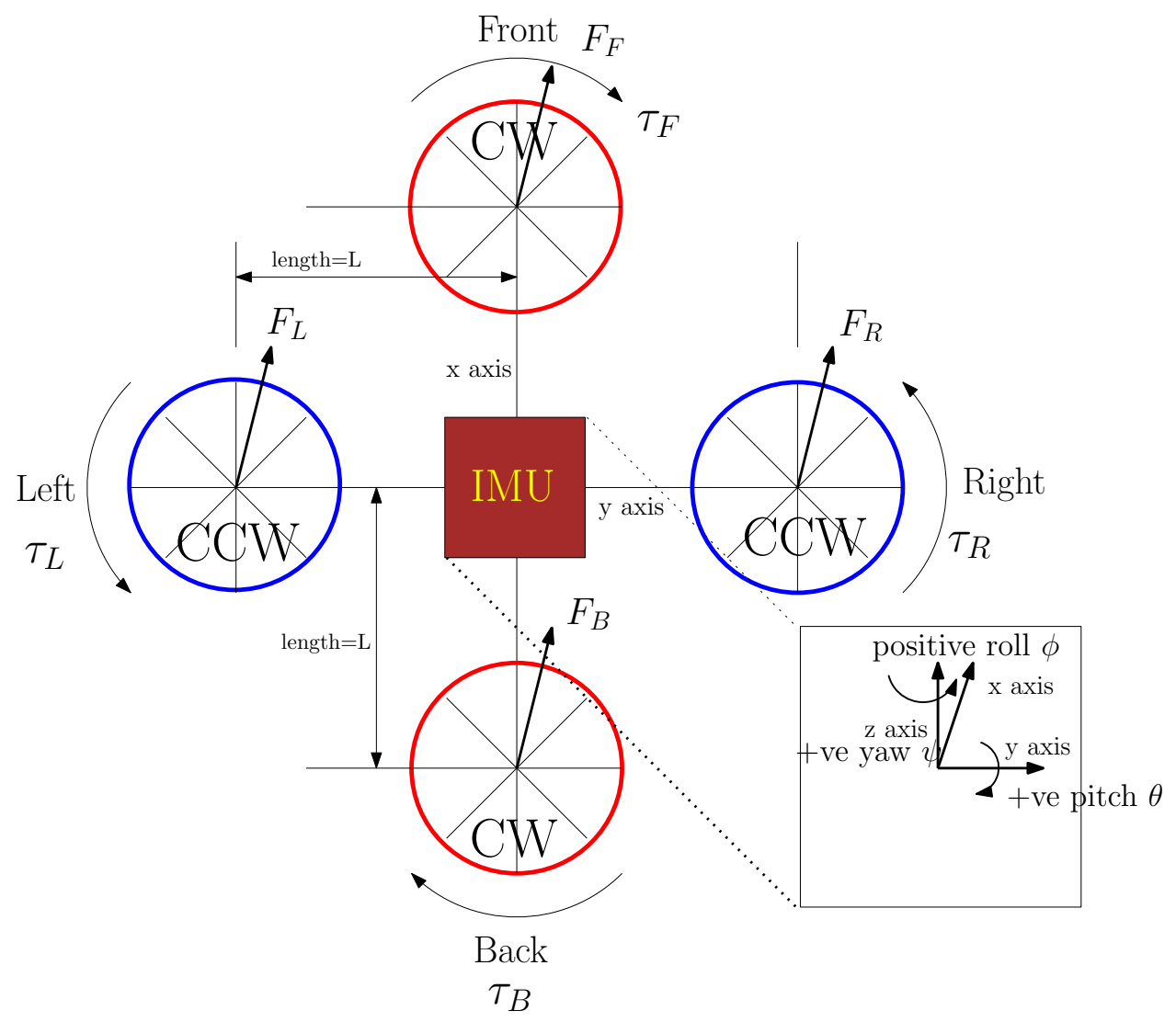

Figure 5.1: Quad-rotor model 


$$
\left[\begin{array}{c}
\ddot{x} \\
\ddot{y} \\
\ddot{z} \\
\ddot{\phi} \\
\ddot{\theta} \\
\ddot{\psi}
\end{array}\right]=\left[\begin{array}{c}
(\cos (\phi) \sin (\theta) \cos (\psi)+\sin (\phi) \sin (\psi)) \frac{T}{M}-\frac{1}{M} k_{d x} \dot{x} \\
(\cos (\phi) \sin (\theta) \sin (\psi)-\sin (\phi) \cos (\psi)) \frac{T}{M}-\frac{1}{M} k_{d y} \dot{y} \\
-g+\cos (\phi) \cos (\theta) \frac{T}{M}-\frac{1}{M} k_{d z} \dot{z} \\
\frac{\tau_{\phi}}{I_{x}} \\
\frac{\tau_{\theta}}{I_{y}} \\
\frac{\tau_{\psi}}{I_{z}}
\end{array}\right]
$$

where $(x, y, z)$ represents the position of a quad-rotor in three dimensional space. Euler angles $(\phi, \theta, \psi)$ represent the angle of rotation about the $x, y$ and $z$ axes respectively in an inertial frame in the anti-clockwise direction. $T$ represents the total thrust due to four rotors in a quad-rotor, where $T=F_{F}+F_{R}+F_{B}+F_{L}=k \sum_{i=1}^{4} \omega_{i}^{2} . F_{F}, F_{B}, F_{R}, F_{L}$ represent force due to rotors, $k$ is a force constant and $\omega_{i}$ refers to the angular velocity of motor $i . k_{d_{x}}, k_{d_{y}}$ and $k_{d_{z}}$ represent aerodynamic drag constants due to velocity along the $x, y$ and $z$ axes respectively. $\tau_{\phi}, \tau_{\theta}$ and $\tau_{\psi}$ are the torques about each rotational axis due to thrust from the propellers, where $\tau_{\phi}=L\left(F_{L}-F_{R}\right)=L k\left(\omega_{1}^{2}-\omega_{3}^{2}\right), \tau_{\theta}=L\left(F_{F}-F_{B}\right)=$ $L k\left(\omega_{2}^{2}-\omega_{4}^{2}\right)$ and $\tau_{\psi}=\tau_{R}+\tau_{L}-\tau_{F}-\tau_{B}=b\left(\omega_{1}^{2}-\omega_{2}^{2}+\omega_{3}^{3}-\omega_{4}^{2}\right) . L$ is half the distance between two opposite rotors, $m$ is mass of the quadcopter and $b$ is the drag constant due to propeller. The moments of inertia of the quadcopter about each respective axis are represented by $I_{x}, I_{y}, I_{z}$. A similar model has been used for the study of controls in [120] but without the aerodynamic forces. Let us represent the states and the controls for a quad-rotor by $X=[x, y, z, \phi, \theta, \psi]^{T}$ and $U=\left[\omega_{1}, \omega_{2}, \omega_{3}, \omega_{4}\right]^{T}$ respectively.

As the angular velocities of the rotors in quad-rotors have saturation point, a limitation in thrust is defined.

$$
T \leq T_{\max }
$$

where $T$ is as defined above, and $T_{\max }>0$.

As suggested by Feldman [87], a point mass model is the most detail model needed for a flight performance problem, as the motion of a vehicle is of interest. And, for our study of trajectory generation for a quad-rotor and its swarm, the interest in on their motion, irrespective of their heading angle. Thus, in order to work with a simple system equation for quad-rotor the complexity of (5.1) was reduced by eliminating the need for heading angle. In the following, the model reduction for a quad-rotor UAV is introduced. 


\section{A reduced dynamic model}

For the problem of trajectory generation for a quad-rotor, an absence of heading angle $\psi$ does not constraint a nature of flight trajectory it could take in $3 D$ space, while $\theta$ and $\phi$ angle are available. As such, Hehn et al. in [41] have suggested to set the rotational rates along $z$-axis to be zero in order to compute controls for their model. Thus, conceivably for the purpose of trajectory generation there is no limitation on the position in space where a quad-rotor could reach by setting the $\psi$ angle and its time derivative to be zero. Therefore, the following assumptions are made,

- Assumption 1: $\psi=0$

- Assumption 2: $\dot{\psi}=0$

- Assumption 3: $\ddot{\psi}=0$

Then using the assumptions, the reduced model for a quad-rotor becomes,

$$
\left[\begin{array}{c}
\ddot{x} \\
\ddot{y} \\
\ddot{z} \\
\ddot{\phi} \\
\ddot{\theta}
\end{array}\right]=\left[\begin{array}{c}
\cos (\phi) \sin (\theta) \frac{T}{M}-\frac{1}{M} k_{d x} \dot{x} \\
-\sin (\phi) \frac{T}{M}-\frac{1}{M} k_{d y} \dot{y} \\
-g+\cos (\phi) \cos (\theta) \frac{T}{M}-\frac{1}{M} k_{d z} \dot{z} \\
\frac{\tau_{\phi}}{I_{x}} \\
\frac{\tau_{\theta}}{I_{y}}
\end{array}\right]
$$

From this reduced model for a quad-rotor there is a possibility to further simplify a system equation for the UAV as indicated in [118], where working with a simpler model for the quad-rotor, there is a possibility to calculate controls to the dynamic model (5.1). In the following a kinematic model is introduced, and an approach to compute controls to the dynamic equation (5.1) using the states and the controls to the kinematic equation is summarized. As stated in the introduction, the purpose of the control calculation is to realize a trajectory during simulation by integrating them in a system model.

\section{A kinematic model}

Let us define a kinematic model for a quad-rotor as follows, 


$$
\left[\begin{array}{c}
\dot{x} \\
\dot{v}_{x} \\
\dot{y} \\
\dot{v}_{y} \\
\dot{z} \\
\dot{v}_{z}
\end{array}\right]=\left[\begin{array}{c}
v_{x} \\
a_{x} \\
v_{y} \\
a_{y} \\
v_{z} \\
a_{z}
\end{array}\right]
$$

where $x, y, z$ are positions in space as defined above, $v_{x}, v_{y}, v_{z}$ are velocities and $a_{x}, a_{y}, a_{z}$ are accelerations along each axes $x, y, z$ respectively. For the kinematic model, $U_{k}=$ $\left[a_{x}, a_{y}, a_{z}\right]$ are control, whereas $X_{k}=\left[x, v_{x}, y, v_{y}, z, v_{z}\right]$ are states variables.

\section{Generation of controls to the dynamic model}

Let us assume a trajectory generation problem is solved for a quad-rotor using kinematic model (5.4). Then, the solution to the kinematic model (5.4), $a_{x}, a_{y}, a_{z}$ as controls and $x, v_{x}, y, v_{y}, z, v_{z}$ as states, are available. Now, let us take the first three equations of (5.3) and re-arrange as follows.

$$
\begin{gathered}
\ddot{x}+\frac{1}{M} k_{d x} \dot{x}=\cos (\phi) \sin (\theta) \frac{T}{M} \\
\ddot{y}+\frac{1}{M} k_{d y} \dot{y}=-\sin (\phi) \frac{T}{M} \\
\ddot{z}+\frac{1}{M} k_{d z} \dot{z}+g=\cos (\phi) \cos (\theta) \frac{T}{M}
\end{gathered}
$$

Since, $\ddot{x}$ and $a_{x}$ both represent acceleration and $\dot{x}$ and $v_{x}$ both represent velocity along $x$-axis, the assumptions $\ddot{x}=a_{x}$, and $\dot{x}=v_{x}$ are made. Similarly along the $y$ and $z$ axes the similar assumption are made resulting in $\ddot{y}=a_{y}, \ddot{z}=a_{z}$ and $\dot{y}=v_{y}, \dot{z}=v_{z}$. Let us define,

$$
\begin{aligned}
& A_{x}=\ddot{x}+\frac{1}{M} k_{d x} \dot{x}=a_{x}+\frac{1}{M} k_{d x} v_{x} \\
& A_{y}=\ddot{y}+\frac{1}{M} k_{d y} \dot{y}=a_{y}+\frac{1}{M} k_{d y} v_{y}
\end{aligned}
$$




$$
A_{z}=\ddot{z}+\frac{1}{M} k_{d z} \dot{z}=a_{z}+\frac{1}{M} k_{d z} v_{z}
$$

Then, (5.5), (5.6) and (5.7) can be written as,

$$
\begin{gathered}
A_{x}=\cos (\phi) \sin (\theta) \frac{T}{M} \\
A_{y}=-\sin (\phi) \frac{T}{M} \\
A_{z}+g=\cos (\phi) \cos (\theta) \frac{T}{M}
\end{gathered}
$$

Now, to calculate the total thrust, both the sides of equations (5.11), (5.12) and (5.13) are squared and added together. After simplification it becomes,

$$
T=M \sqrt{A_{x}^{2}+A_{y}^{2}+\left(A_{z}+g\right)^{2}}
$$

From equation (5.12), $\phi$ can be solved as,

$$
\phi=\arcsin \left(\frac{-A_{y}}{\sqrt{A_{x}^{2}+A_{y}^{2}+\left(A_{z}+g\right)^{2}}}\right)
$$

And, from equations (5.11) and (5.13), $\theta$ can be solved as,

$$
\theta=\arctan \left(\frac{A_{x}}{A_{z}+g}\right)
$$

Although a slight variation of expressions for $T, \phi$ and $\theta$ are also derived in [118] for the problem of trajectory tracking, our derivation can be considered as a reduced expression for the problem of our nature. Now, since $\theta$ and $\phi$ are available, $\ddot{\theta}$ and $\ddot{\phi}$ can be numerically computed by taking time derivative as,

$$
\begin{aligned}
& \dot{\theta}=\frac{d \theta}{d t} \text { and } \ddot{\theta}=\frac{d \dot{\theta}}{d t} \\
& \dot{\phi}=\frac{d \phi}{d t} \text { and } \ddot{\phi}=\frac{d \dot{\phi}}{d t}
\end{aligned}
$$


Since, $\theta$ and $\phi$ are computed from the trajectory (using the kinematic model), its time derivative depends on the trajectory as well. As of now, thrust $(T), \ddot{\theta}$ and $\ddot{\phi}$ are available and $\ddot{\psi}=0$ (by Assumptions 1,2 and 3). The last three equations of (5.1) can be written as,

$$
\left[\begin{array}{c}
\tau_{\phi} \\
\tau_{\theta} \\
\tau_{\psi}
\end{array}\right]=\left[\begin{array}{c}
\ddot{\phi} I_{x} \\
\ddot{\theta} I_{y} \\
\ddot{\psi} I_{z}
\end{array}\right]
$$

Now, the expressions for thrust, and torques can be written in terms of the control inputs $w_{i}^{2}$ where $i=1,2,3,4$ as,

$$
\left[\begin{array}{c}
\omega_{1}^{2}+\omega_{2}^{2}+\omega_{3}^{2}+\omega_{4}^{2} \\
\omega_{1}^{2}-\omega_{3}^{2} \\
\omega_{2}^{2}-\omega_{4}^{2} \\
\omega_{1}^{2}-\omega_{2}^{2}+\omega_{3}^{3}-\omega_{4}^{2}
\end{array}\right]=\left[\begin{array}{c}
\frac{T}{k} \\
\frac{\tau_{\phi}}{L k} \\
\frac{\tau \theta}{L k} \\
\frac{\tau_{\psi}}{b}
\end{array}\right]
$$

After simplification the expression for the control inputs to the dynamic model can be written as follows [119].

$$
\begin{aligned}
& \omega_{1}^{2}=\frac{T}{4 k}+\frac{\tau_{\phi}}{2 L k}+\frac{\tau_{\psi}}{4 b} \\
& \omega_{2}^{2}=\frac{T}{4 k}+\frac{\tau_{\theta}}{2 L k}-\frac{\tau_{\psi}}{4 b} \\
& \omega_{3}^{2}=\frac{T}{4 k}-\frac{\tau_{\phi}}{2 L k}+\frac{\tau_{\psi}}{4 b} \\
& \omega_{4}^{2}=\frac{T}{4 k}-\frac{\tau_{\theta}}{2 L k}-\frac{\tau_{\psi}}{4 b}
\end{aligned}
$$

Hence, once the trajectory generation problem using the kinematic model is solved, its solution is used to find the controls for the dynamic model using equations (5.8)(5.21). The rotors speed are used as inputs to the quad-rotor dynamic model (5.1) for Runge-Kutta integration in order to realize a trajectory during simulation. Although, a trajectory generated from a trajectory generation problem could directly be used as input to an autopilot like system so that a low-level controller within the system may track 
the trajectory. The control inputs to the model (5.1) which are rotors speed may as well be directly used for implementation which becomes a model predictive control (MPC) onboard controller, but it may arise a concern with robustness depending on the speed of the model predictive control (MPC) controller. However, this thesis is only concern with a high level trajectory generator, and the low level flight controller is beyond the scope of this thesis.

\subsubsection{Problem Definition}

In order to compare trajectories between the use of the kinematic and the dynamic model for a quad-rotor UAV a trajectory generation problem is defined. This problem aims to compare the nature of trajectories generated and their associated computation times between these two models, (5.3) and (5.4). Since, the solution time, the cost of a trajectory generated, and the avoidance of obstacles play vital roles in deciding the quality and safety of a quad-rotor UAV, the comparison between the kinematic and the dynamic models is made under the same grounds; solution time and the cost of a trajectory with obstacle avoidance.

The problem of trajectory generation considered is similar to the problem definition as in Chapter 4, with the same cost function but with constraints for a quad-rotor. Thus, as similar to the cost function from Chapter 4 that measures deviation from a reference path, robustness from obstacles, and a term that measures progress along the desired direction of flight, the cost function is rewritten as,

$$
J(X(\cdot), U(\cdot))=\int_{\tau_{0}}^{\tau_{f}}\left[\alpha\left(y(t)-y_{d}\right)^{2}+\beta\left(z(t)-z_{d}\right)^{2}+\eta\left(x(t)-x_{a}\right)^{2}+\bar{r}(X(\cdot))\right] d t
$$

where all the terms are as stated in Section 4.2.3.

Now, a trajectory generation problem using the dynamic model is defined as, given an initial condition $\left(x_{0}, y_{0}, z_{0}\right)$ at time $\tau_{0}$, find the control-state pair $(X(t), U(t))$ to minimize the cost (5.22) subject to 


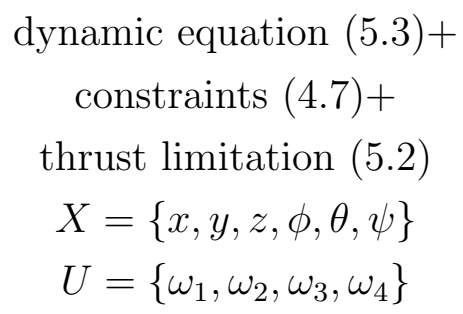

Similarly, a trajectory generation problem using a kinematic model is defined as, given an initial condition $\left(x_{0}, y_{0}, z_{0}\right)$ at time $\tau_{0}$, find the control-state pair $\left(X_{k}(t), U_{k}(t)\right)$ to minimize the cost (5.22) subject to

$$
\begin{gathered}
\text { kinematic equation }(5.4)+ \\
\text { constraints }(4.7)+ \\
M \sqrt{A_{x}^{2}+A_{y}^{2}+\left(A_{z}+g\right)^{2}} \leq T_{\max } \\
X_{k}=\left\{x, y, z, v_{x}, v_{y}, v_{z}\right\} \\
U_{k}=\left\{a_{x}, a_{y}, a_{z}\right\}
\end{gathered}
$$

where the constraint $T \leq T_{\max }$ in (5.2) has been replaced by $M \sqrt{A_{x}^{2}+A_{y}^{2}+\left(A_{z}+g\right)^{2}} \leq$ $T_{\max }$ in the kinematic model, where $T$ for the kinematic model has been defined in (5.14) ([41] used similar constraint to limit the acceleration of a quad-rotor UAV). Once the problem (5.24) is solved, equations (5.8)-(5.21) are used to compute controls to the dynamic model.

The above problems in this chapter are solved by the method of finite horizon model predictive control as in Section 4.3. In order to solve the problems, the method of sequential convex programming ( $\mathrm{SCP}$ ) as in Section 2.2 is used, where a custom solver from Section 2.3 is used to solve a quadratic programming (QP) problem within the sequential convex programming (SCP) approach.

\subsection{Numerical Examples}

\subsubsection{Choice of $\Delta t$}

The time-step $\Delta t$ is used to approximate the system dynamics at discrete time. The choice of $\Delta t$ in a problem discretization determines the accuracy of system approximation 
[117], as well as the problem size if the finite horizon $\tau_{f}$ is fixed. Section 4.4 .2 provides a summary on the effect of $\Delta t$ on the problem size and the accuracy of solution.

As similar to Section 4.4.2, the trajectories of a quad-rotor with $\Delta t=300,500,1500$ milliseconds between the problem using the kinematic and the dynamic model are compared. For the comparison a scenario with a cuboid shaped stationary obstacle is considered, which has square base with 20 meters edge and centered at $(60,5)$ meter in the $x-y$ plane, and the height of 30 meters. A quad-rotor UAV starts from $(0,0,20)$ meters, with its initial velocities, pitch and roll angles as zero, and is desired to fly along the positive $x$-axis at the height of 20 meters, with $x_{a}$ as 240 meters, thus $y_{d}=0$ meter and $z_{d}=20$ meters. All the parameters for the UAV are given in the Appendix 4.1 where applicable and the remaining problem parameters are given in Table 5.1.

Table 5.1: Problem parameters

\begin{tabular}{cccc}
\hline \hline Parameter & Value (unit) & Parameter & Value (unit) \\
\hline$\alpha, \beta$ & $0.5,0.5$ & $\Delta t$ & $(0.3,0.5,1.5)$ (second) \\
$\eta$ & 0.1 & $x_{a}$ & 240 (meter) \\
{$\left[\tau_{0}, \tau_{f}\right]$} & {$[0,6]$ (second) } & $T_{\max }$ & $2 \cdot M \cdot g$ (newton) \\
\hline \hline
\end{tabular}

As shown in the table, the penalty parameters $\alpha$ and $\beta$ both have been set to 0.5 , and $\eta$ to 0.1 . Since the aim is to remain close to a reference path more weight is assigned to that, while for progressing towards the desired direction of flight, a low penalty weight is set. However, robustness for obstacle avoidance is also a priority, thus, the penalty associated with robustness function increases to maximum when a UAV approaches an obstacle and the penalty goes to zero when the UAV is far apart from obstacles. The problem is solved using the method of finite horizon model predictive control as outlined in 4.3, using either (5.23) or (5.24) as the finite horizon optimal control problem. Using the approach, the problems are solved with a finite horizon $\left(\tau_{f}\right)$ of 6 seconds with various sampling time (given). Similarly, the maximum allowable thrust $\left(T_{\max }\right)$ has been set to $2 \times M \times g$ which is two times mass $(M)$ times acceleration due to gravity $(g)$.

Figure 5.2 shows the trajectories obtained from solving problem (5.23) within the model predictive control (MPC) framwork, with various $\Delta t$ and a fixed-finite horizon of $\tau_{f}=6$ seconds. As seen in the figure, trajectory gets better in terms of remaining close to the reference path with decreasing $\Delta t$. The trajectories as seen in the figure are the outcome of integrating control inputs into the system dynamics (5.1), where the inputs 
are generated by solving (5.23) within an model predictive control (MPC) framework, and the inputs up to the control horizon of $\Delta t$ are integrated in the system dynamics at each model predictive control (MPC) instant. It can be seen that the trajectories using the dynamic model get deviated from the reference path with $\Delta t$ of 1500 milliseconds in comparison with the remaining $\Delta t$. It is as expected, due to the fact that linearization of a model deviates from the actual dynamic model, especially when the original model is highly nonlinear. In the figure, the trajectory with $\Delta t=300$ milliseconds remains close to the reference path for almost all the time except while avoiding the obstacle. Although, the trajectory with $\Delta t=500$ milliseconds is close to the reference path as well, slight deviations can be seen for the trajectory when compared to the trajectory with $\Delta t=300$ milliseconds.

Figure 5.3 shows the trajectories obtained by integrating the solution from problem (5.24), to the dynamic model (5.1). Although, a quad-rotor can avoid an obstacle, and move forward along the desired direction of flight, the decrease in discretization time steps for the given finite horizon improves the trajectory in terms of avoiding obstacles and remaining close to the reference path. The figures in 5.3 shows that the trajectory with $\Delta t=300$ milliseconds remains close to the reference path as similar to the case with the dynamic model. However, trajectories from $\Delta t=500$ and $\Delta t=1500$ milliseconds deviate from the reference path.

In both Figures 5.2 and 5.3, trajectories with $\Delta t=300$ milliseconds seems virtually close and similar (except while avoiding obstacles, compare figures $5.2 \mathrm{~b}$ and $5.3 \mathrm{~b}$ ). In addition, to compare the trajectories around the obstacle, their deviation around the stationary obstacle is computed using equation (4.20) (from $x=20$ to $x=100 \mathrm{~m}$ ) and then average the cost by the number of sampling points in between $x=20$ to $x=100 \mathrm{~m}$. It was found that the average deviations for the problem using the kinematic model with $\Delta t=[300,500,1500]$ milliseconds are $[5.91,6.54,9.77]$ respectively, while for the case of the dynamic model they are $[5.95,6.35,7.70]$ respectively. Thus, from the deviation comparison it was found that the average deviations for $\Delta t=300$ milliseconds are close for both problems (5.23), and (5.24). Hence, from the average deviation comparison, and the visual inspection from Figures 5.2 and 5.3, the trajectories using the models for the UAV with $\Delta t=300$ milliseconds are found comparable. Therefore in the next subsection a comparative study between the problems defined in Section 5.2.2 is presented, where $\Delta t=300$ milliseconds is used(for Problems (5.23), and (5.24)). The reason such sampling 

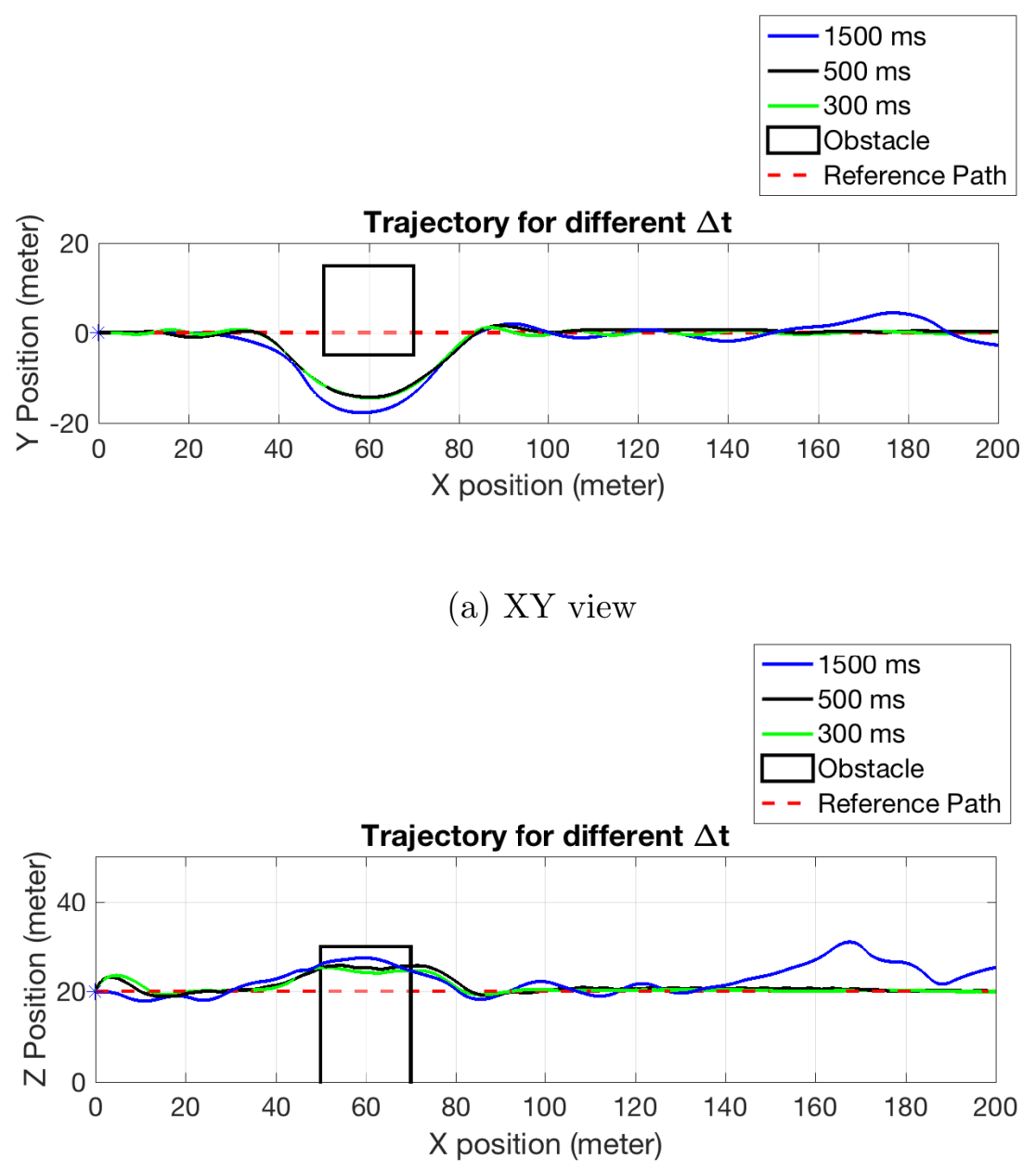

(b) XZ view

Figure 5.2: Trajectories using the dynamic model for different values of $\Delta t$ 

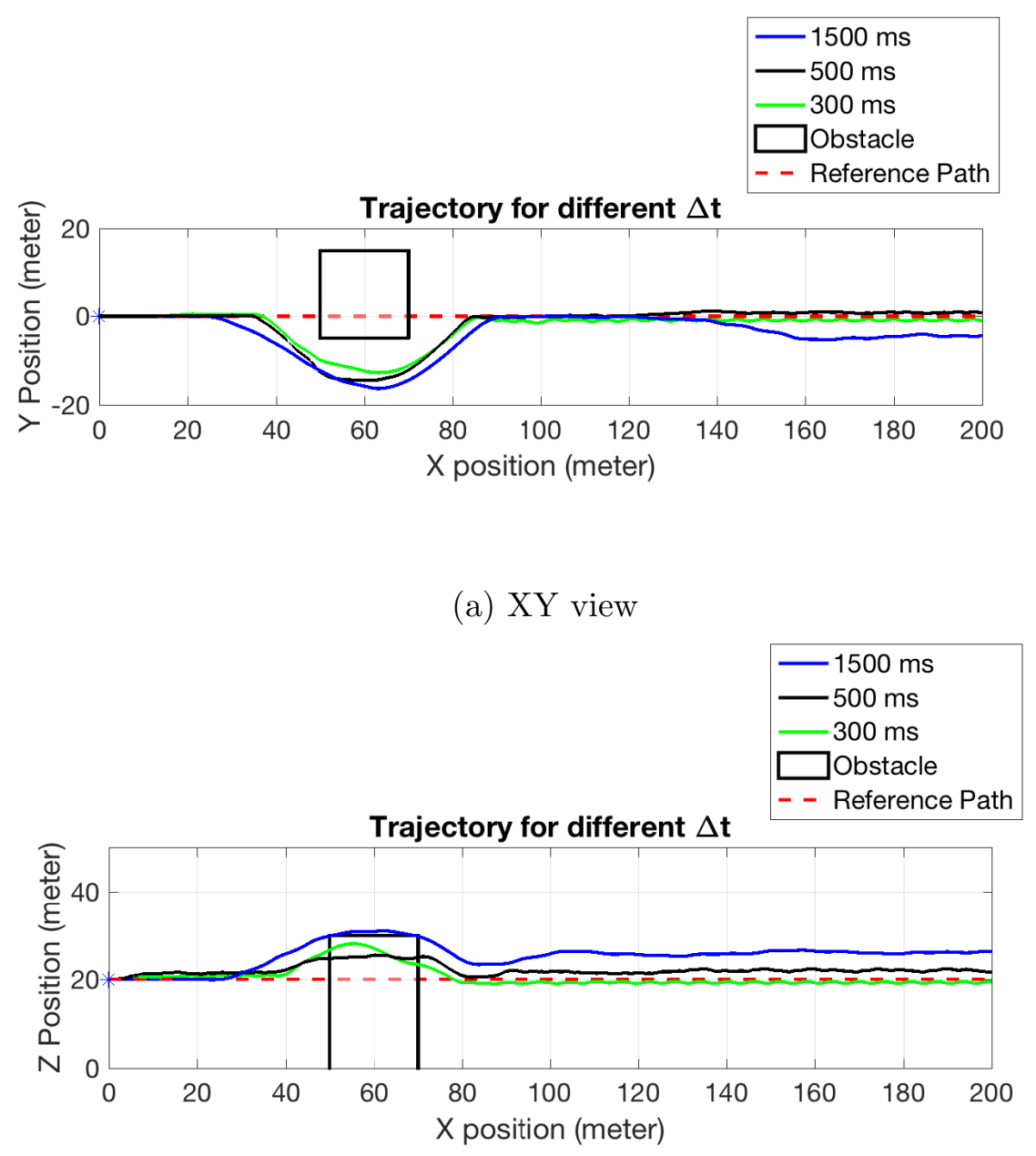

(b) XZ view

Figure 5.3: Trajectories using the kinematic model for different values of $\Delta t$ 
time is chosen for the problems is to compare over the similar quality of trajectory as seen from the above.

\subsubsection{Scenario with Obstacles}

In this section, trajectories from problems (5.23) and (5.24) are compared in terms of a quality of the trajectories (that remains close to a reference path and avoids obstacles) and the computation time. While the optimization parameters (the number of states and controls) for the problems vary, their aim is to solve for a trajectory that a quad-rotor takes from an initial point towards the desired direction of flight by avoiding obstacles while remaining close to a reference path.

For both the problems, the quad-rotor parameters are taken from the Appendix 4.1, bounds on optimization variables as stated in Appendix 4.2, and obstacle parameters from the table in Appendix 4.4. For these problems seven obstacles are used among which two of them are moving obstacles (refer to (4.7) for obstacle constraint). The first moving obstacle starts from $(130,0,20)$ meter and goes along the positive $y$ direction with the velocity of $6 \mathrm{~m} / \mathrm{s}$, while the second starts at $(100,300,20)$ meters and heads towards the negative $y$ - axis with velocity $-7.5 \mathrm{~m} / \mathrm{s}$. Table 5.1 has the additional parameters that are used for the comparative study, such as $\alpha, \beta, \eta, x_{a}, T_{\max }$, and the finite horizon length, $\tau_{f}$. Thus, using the $\tau_{f}=6$ seconds, and sampling time $(\Delta t)$ of 300 milliseconds for the Problems, the problem will have $N=20$ discretization points. An initial point for the quad-rotor $\operatorname{UAV}\left(x_{0}, y_{0}, z_{0}\right)$ is set at $(0,150,20)$ meter, with initial velocities, roll and pitch angles as zero, along with $y_{d}$ as 150 meters, $z_{d}$ as 20 meters. Finally, the trajectory generation is terminated once the UAV reaches $x=200$ meters.

The problems are solved using the method of Finite Horizon Model Predictive Control as outlined in 4.3, using either (5.23) or (5.24) as the finite horizon optimal control problem solved at each model predictive control (MPC) update. As mentioned, a custom quadratic programming (QP) solver is used to solve the quadratic programming (QP) sub-problems. The trajectory generation problem with the dynamic model has 8206 nonzero entries in the Karush-Kuhn-Tucker (KKT) matrix within its quadratic programming (QP) subproblem whereas for the case of the kinematic model the number of nonzero entries in its quadratic programming (QP) subproblem is 4757. Consequently, the number of floating point operations required between these two problem formulation 
differs that leads to the difference in the computational complexity ([62] Appendix C.1), and it should be clearly reflected in the solution time which is presented below.

Figure 5.4 compares the trajectories obtained by solving problems (5.23) and (5.24). In the figure, two moving obstacles are shown with their velocities along side and their direction of flight. Since, the problems in comparison have different optimization parameters they may not necessarily have exactly the same trajectory, however, the trajectories are very close.

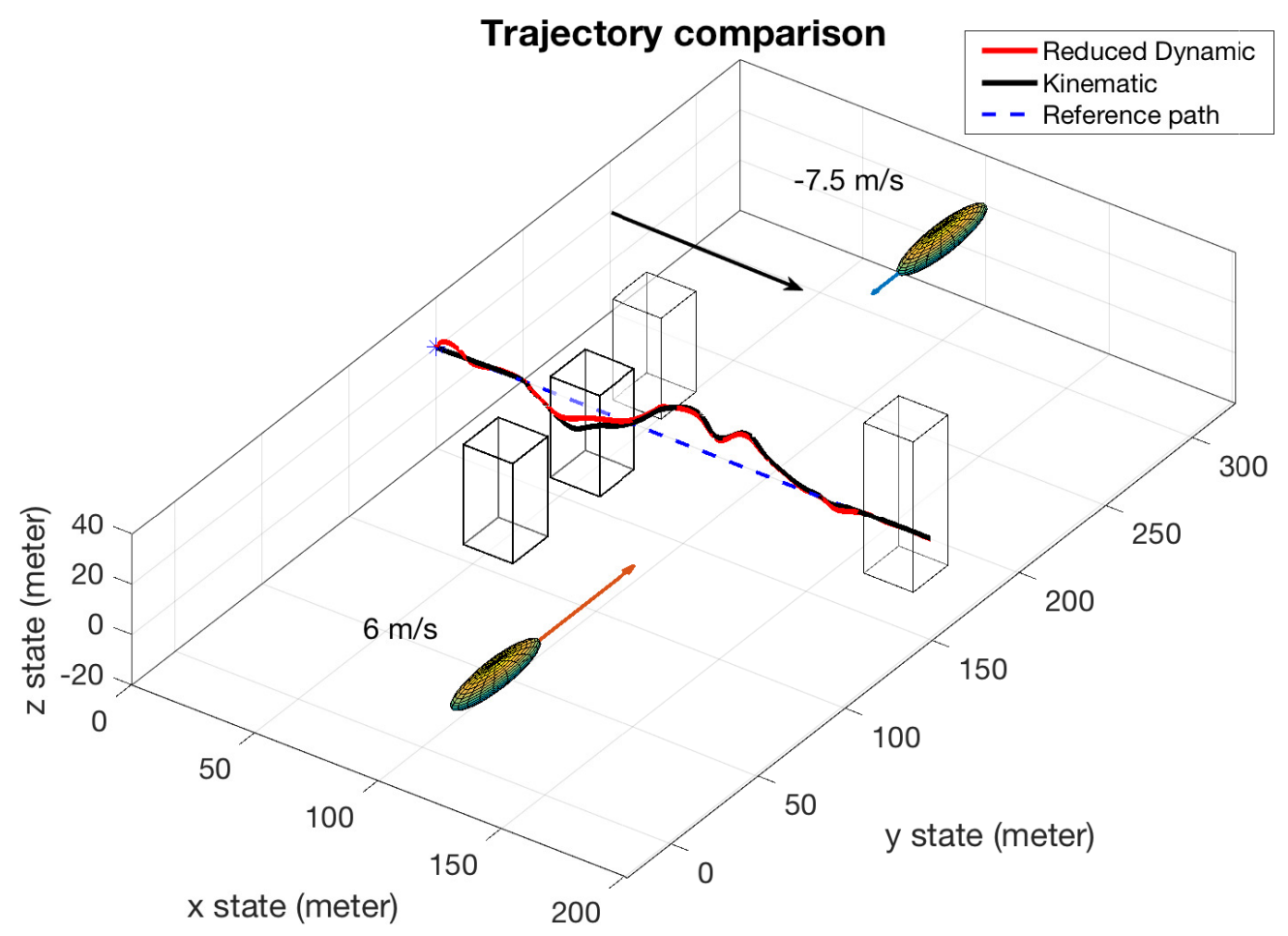

Figure 5.4: Trajectory comparison, 3D view

Likewise, Figure 5.5 shows the XY view of the figure 5.4 in which both the trajectories appear to be overlapped except at around $x=160$ meters, however the deviation between them is not much significant. In the XY view, moving obstacles are hidden and the view is zoomed so as to see the deviation of the trajectory from the reference path.

Figure 5.6 shows the XZ view of the figure 5.4 which is zoomed at around moving obstacles. As similar to above figures in this subsection, the trajectories appear very 


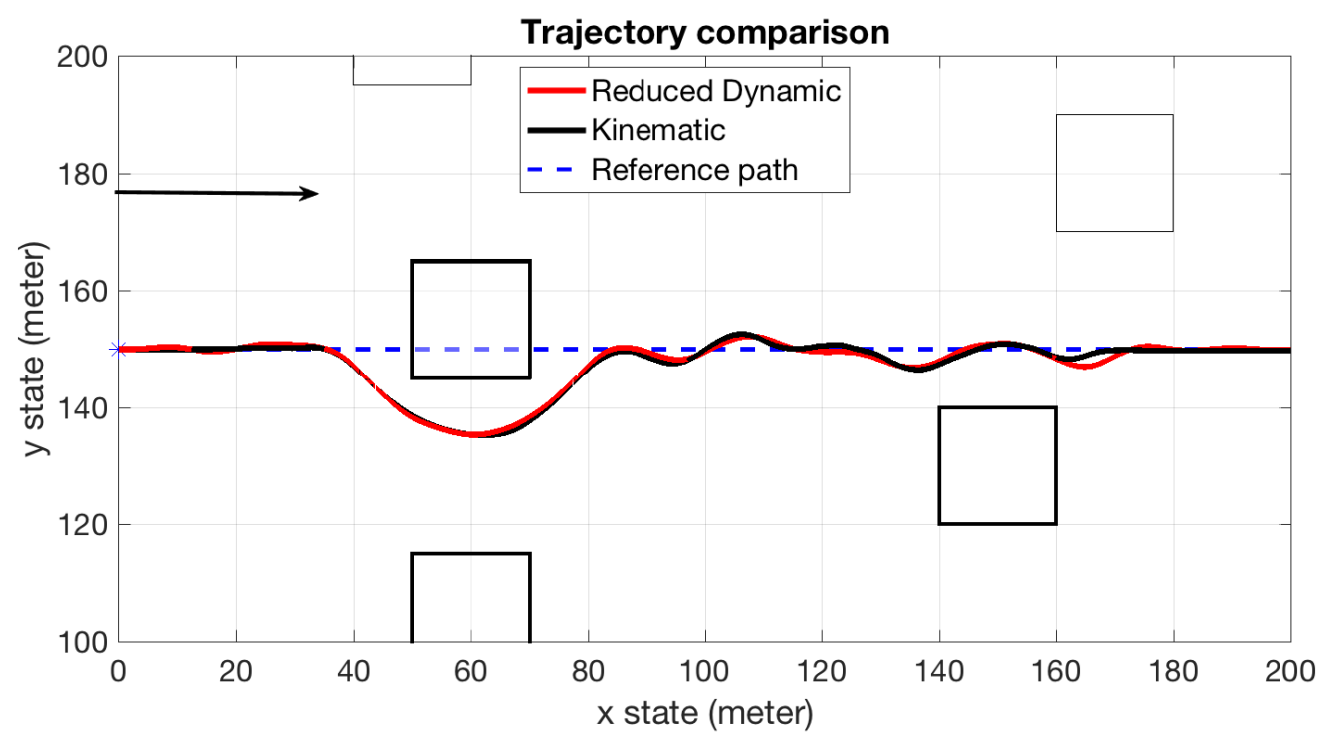

Figure 5.5: Trajectory comparison, $\mathrm{XY}$ view

close to each other.

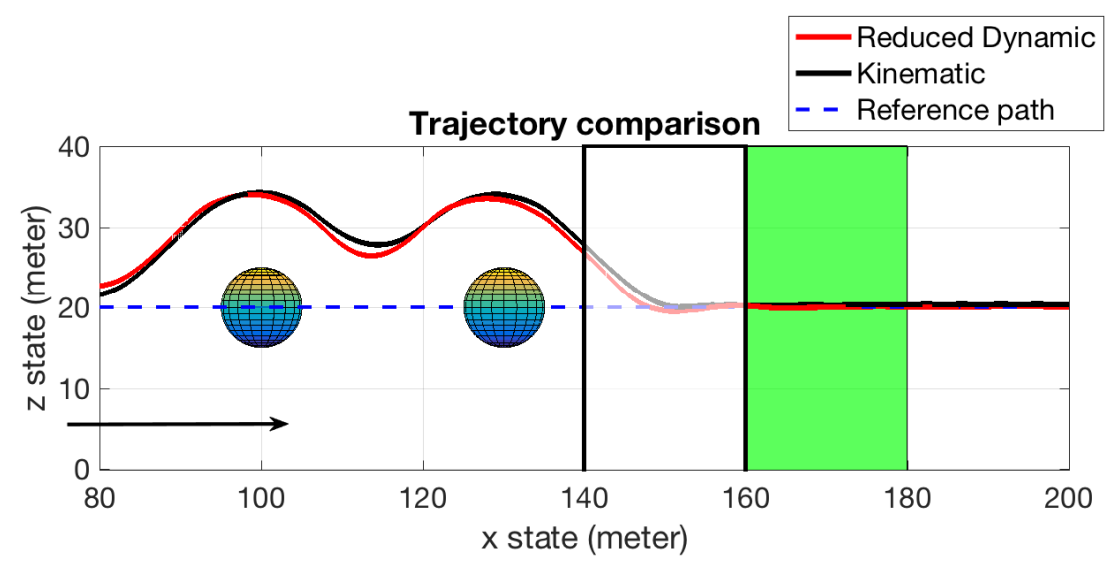

Figure 5.6: Trajectory comparison around moving obstacles, XZ view

After the problems (5.23) and (5.24) are solved using a custom solver (using MEX-file from Matlab in a laptop computer with $1.8 \mathrm{GHz}$ processor and 4GB RAM), Figure 5.7 shows the computation time for both the problems. As expected, the computation time for the kinematic model is always lower while compared to the problem with the dynamic model. It is due to the fact that the kinematic model has less number of optimization 
parameters compared to the dynamic model. The figure shows the computation time in logarithm plot along $y$-axis for clear comparison. The maximum computation time using the kinematic model is 6.7 milliseconds while the minimum computation time using the dynamic model is 11.57 milliseconds. Likewise, the maximum computation time using the dynamic model is 43.24 milliseconds. Similarly, if an average solution time is to be compared then the reduced dynamic model takes 36.5 milliseconds shown by red dashed line, while the kinematic model takes 3.3 milliseconds shown by black dashed line.

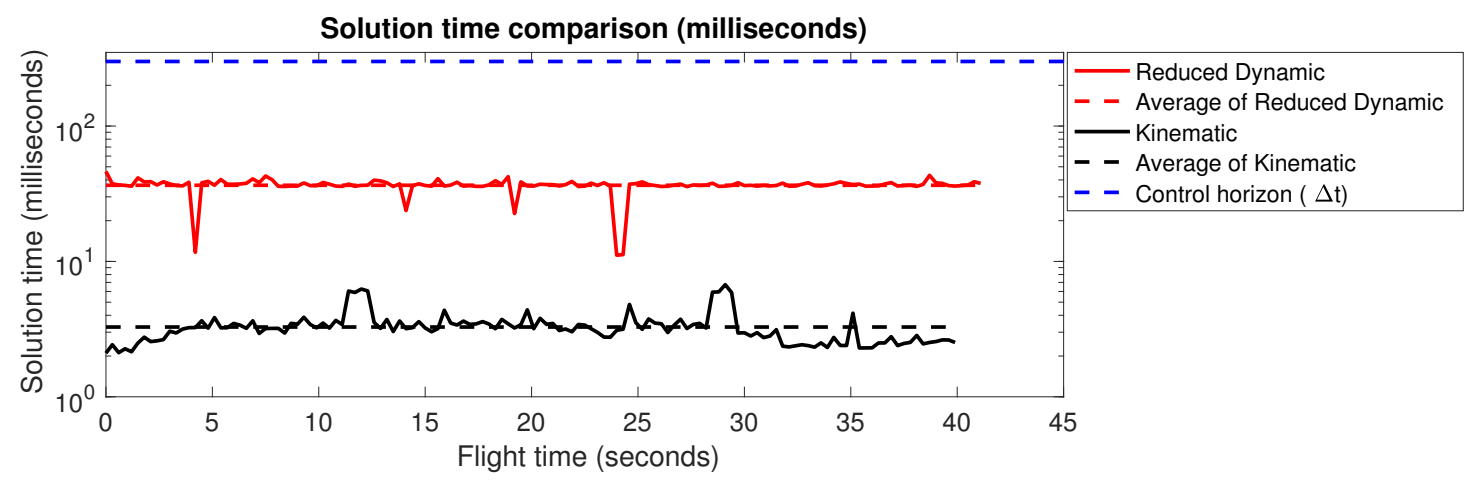

Figure 5.7: Computation time comparison

Next, the computation time on a less capable processor is examined, such as would be available on a UAV. Figure 5.8 shows the computation time for the problems on a Raspberry PI 3B computer (1.2GHz Broadcom CPU and 1GB RAM) [95] which uses an embedded solver. The maximum and the minimum computation time to solve (5.23) at a given time instance in the embedded platform are 229.92 milliseconds and 77.25 milliseconds respectively, whereas the maximum and the minimum computation time to solve (5.24) at a given time instance in the platform are 40.82 milliseconds and 29.96 milliseconds respectively. Although, the maximum solution time using the dynamic model is within the chosen sample time which may be suitable (accounting other factors such as communicating with an autopilot) for real-time implementation according to the sample period of 300 milliseconds, the kinematic model offers approximately twice the lower solution time than the dynamic model. Hence, the kinematic model offers more margin of safety in terms of solution time which is 7.5 times lower than the chosen sample period of 300 milliseconds. However, solution time in the computer can be hugely affected by the change in problem parameters including the number of obstacles in a problem, temperature of the CPU and other system program initiated by the operating system within 
the computer. Moreover, the solution time is not the hard-real time. In Figure 5.8, the jump in the solution time is seen after 15 seconds of flight time which becomes constant afterward as there is a fixed number of floating point operation to be performed using custom solver. In terms of average time, the reduced dynamic model takes 176.02 milliseconds shown by red dashed line, while the kinematic model takes 31.24 milliseconds shown by black dashed line, using the ARM-based computer.

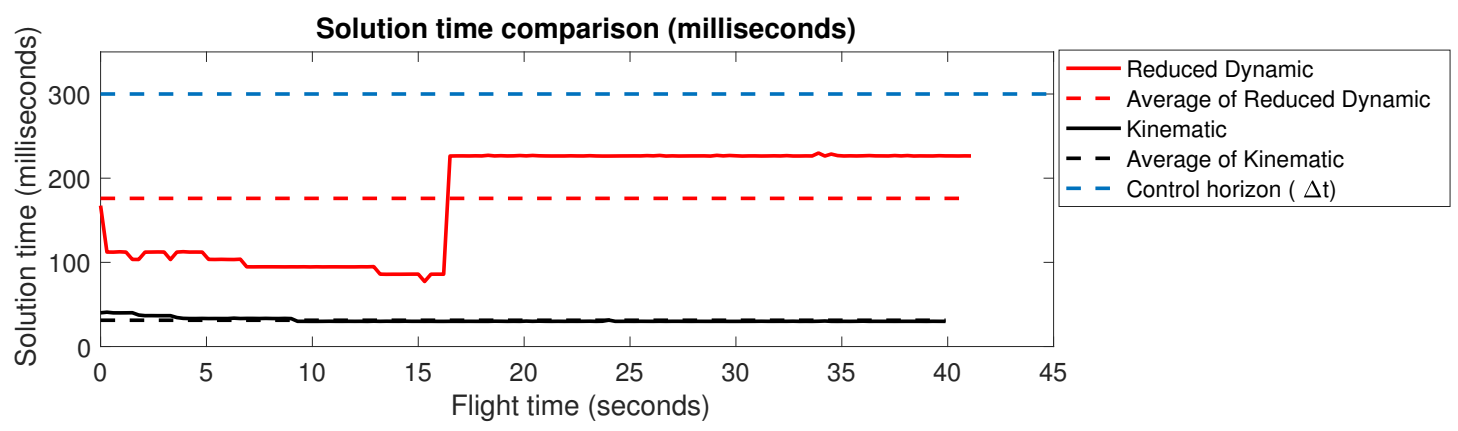

Figure 5.8: Computation time comparison in Raspberry PI 3B

Figure 5.9 shows the thrust calculated by both the problems (5.23) and (5.24). In the figure, the blue dashed line shows the limit as defined in the table 5.1, where the blue dashed line represent $2 \cdot M \cdot g$, which is the maximum allowable thrust for both models. The thrust limitation constraint is given by (5.2). From the figure, it can be seen that both the problems take similar ranges of thrust. However, on average the reduced dynamic model uses 5.75 newton thrust shown by red dashed line, while the kinematic model needs 5.11 newton thrust shown in the figure by black dashed line.

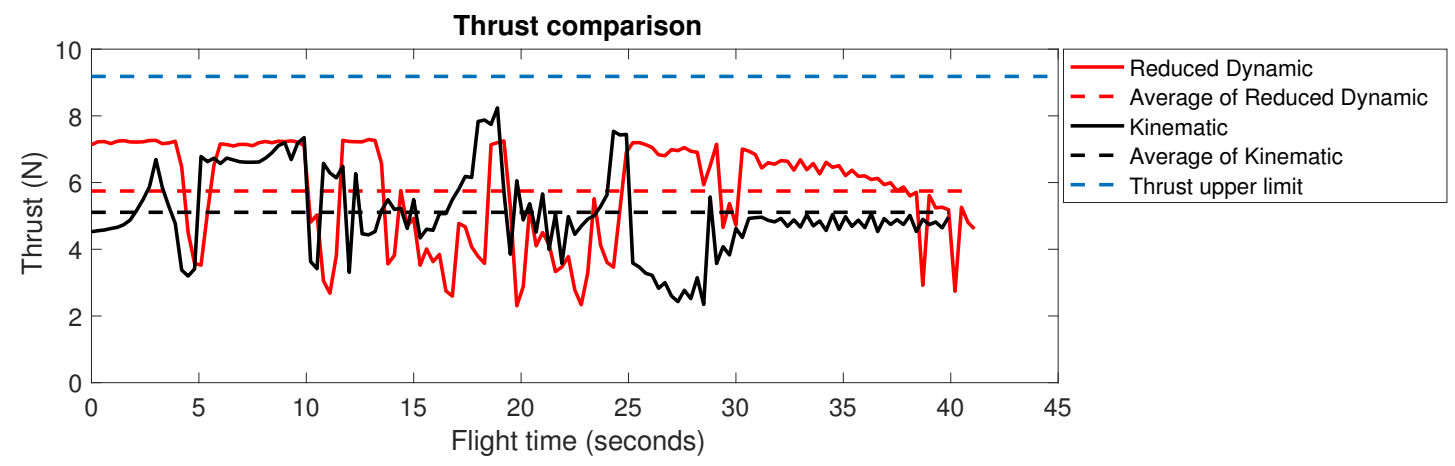

Figure 5.9: Thrust required for trajectories

Hence, from the fact that the problem with the kinematic model has fewer optimiza- 
tion parameters, the problem takes less computation time (see figure 5.7), and thus has more chances of real-time usability, however, both the problems provide similar quality of trajectories for similar problem parameters. In the subsection below, a further study is performed on the difference between the use of the kinematic and the dynamic model for quad-rotor trajectory generation by the Monte-Carlo test.

\section{Comparison with ECOS and IPOPT}

As similar to Section 4.4.1, the trajectory generation with other solvers (ECOS and IPOPT) is compared for the scenario in Section 5.3.2. The problems of trajectory generation using the Kinematic and the Dynamic model for quadrotor are compared in terms of cost and the computation time.

Table 5.2 shows the comparison between the solvers for the scenario above using the Kinematic model for the quad-rotor UAV. In the comparison, the CS solver not only solves fastest but also takes the least deviation among other solvers. Although, IPOPT takes the maximum computation time among the solvers, it's value of cost is very close to that of CS, while ECOS deviates more than other two.

Table 5.2: Comparison of the custom solver (CS) with IPOPT and ECOS: For trajectory with Kinematic model.

\begin{tabular}{cccc}
\hline \hline & Cost & Average computation time $(\mathrm{ms})$ & Maximum computation time (ms) \\
\hline IPOPT & 11.7 & 378.7 & 990.4 \\
\hline ECOS & 15.2 & 13.3 & 21.2 \\
\hline CS & 11.6 & 3.3 & 6.7 \\
\hline \hline
\end{tabular}

Table 5.3 shows the comparison between the solvers for the scenario using the Dynamic model for the quad-rotor UAV. As similar to the above case, the CS deviates the least with the lowest maximum computation time. Nevertheless, ECOS takes the lowest average computation time. Although the cost from IPOPT is similar to that of CS, but the solver is computationally the most intensive among the three.

\subsubsection{Monte-Carlo Test}

The above example for a quad-rotor UAV may not be sufficient to compare the models for real-time trajectory generation and autonomous obstacle avoidance. Therefore, in this 
Table 5.3: Comparison of the custom solver (CS) with IPOPT and ECOS: For trajectory with Dynamic model

\begin{tabular}{cccc}
\hline \hline & Cost & Average computation time $(\mathrm{ms})$ & Max computation time $(\mathrm{ms})$ \\
\hline IPOPT & 13.4 & 451.1 & 1947.8 \\
\hline ECOS & 16.0 & 25.8 & 76.2 \\
\hline CS & 12.7 & 36.5 & 43.2 \\
\hline \hline
\end{tabular}

subsection Monte-Carlo test is performed with the same problem defining parameters as the above example, but with various obstacle scenarios. As similar to the Monte-Carlo example in Section 4.4.3, 100 tests are performed in which obstacles and their parameters (parameters define obstacle's location, shape, and size) are randomly initialized with uniform distribution. In the test, the number of obstacle range from $2 \sim 20$, the parameters that define an obstacle size in terms of centre distance to surface $\left(a_{i}, b_{i}\right.$, and $c_{i}$ in (4.7)) range from $20 \sim 50$ meters, and the parameters that define an obstacle shape $\left(p_{x}, p_{y}\right.$, and $p_{z}$ in (4.7)) range from $2 \sim 10$ (all of these parameters randomly initialized with uniform distribution). For a scenario, obstacles are distributed in the space between $100 \sim 700$ meters along the positive $x$-axis (not at 0 so as to avoid obstacles near the initial point), $-300 \sim 300$ meters along the y-axis and $0 \sim 400$ meters along the z-axis. In any scenario, at least a stationary and a moving obstacles are initialized with maximum of 20 obstacles. The parameters defining the nature of moving obstacles are the same as in Section 4.4.3. For any scenario, a quad-rotor UAV starts from the point $x=0, y=150$ meters at the height of $z=20$ meters and flies along the positive $x$-axis. In order to have a meaningful way to terminate the simulation a point is assigned along the desired reference path where the simulation is stopped. As similar to the use of $x_{a}$ in Section 5.3 .2 , the simulation is stopped once the UAV reaches $x=750$ meters (but use $x_{a}=800$ meters for the Monte-Carlo test). The results are summarized in the followings.

Figure 5.10 shows the solution time taken by the custom solver to solve for the model predictive control (MPC) problem at each model predictive control (MPC) time instant (at a given time $\mathrm{t}$ before moving to the next model predictive control (MPC) window) throughout all the scenarios. For more than $95 \%$ of the instances it takes less than or equal to 5 milliseconds of solution time for the kinematic model, where as for the case of the dynamic model the model predictive control (MPC) problem takes about 15 to 20 milliseconds solution time for approximately $93 \%$ of the instances. There are less 
than 5\% of instances where the kinematic model takes 5-10 milliseconds solution time, whereas for the case of dynamic model approximately $2.5 \%$ of instances takes more than 20 milliseconds of solution time. However, the problem with the dynamic model always takes less than or equal to 25 milliseconds of solution time.

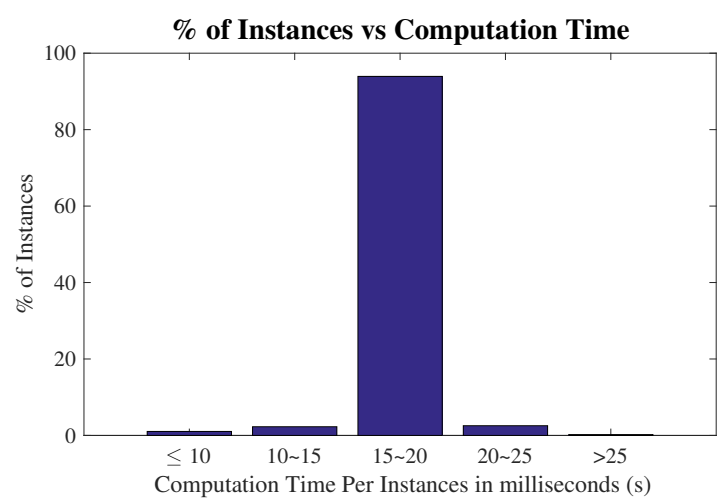

(a) The case of dynamic model

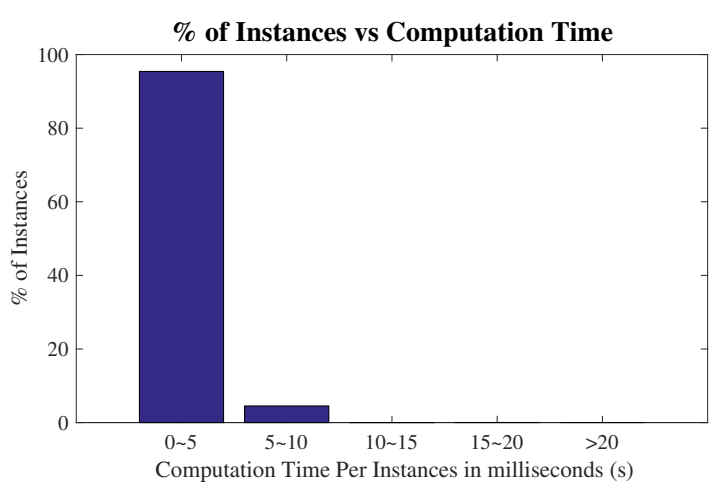

(b) The case of kinematic model

Figure 5.10: Computation time per instant for all scenarios

In order to measure the deviation of trajectories from the reference path, the expression in (4.20) is used, that finds the total value of the square of deviation for each scenario at states history traced out by the quad-rotor. The obstacles are distributed between $x=100 \sim 700$ meters, therefore, only the part of the trajectory that requires avoidance of obstacles is measured. Since, an obstacle could have the maximum radius of 50 meters, therefore, the square of deviation is measured between $x=50 \sim 750$ meters. The figures in 5.11 show the value of the square of deviation. From figures 5.11a and $5.11 \mathrm{~b}$, it can be observed that both the dynamic and the kinematic models for almost the same percentages of scenarios take similar ranges of values for the square of deviation until $10 \times 10^{5}$ which accumulates to $95 \%$ of scenarios. For the deviation range of $10 \times 10^{5}$ to $20 \times 10^{5}$ the dynamic model has $1 \%$ of scenarios while the kinematic model has $2 \%$ of scenarios. However, for the deviation range above $20 \times 10^{5}$ the kinematic model has $2 \%$ of scenarios less than the dynamic model. 


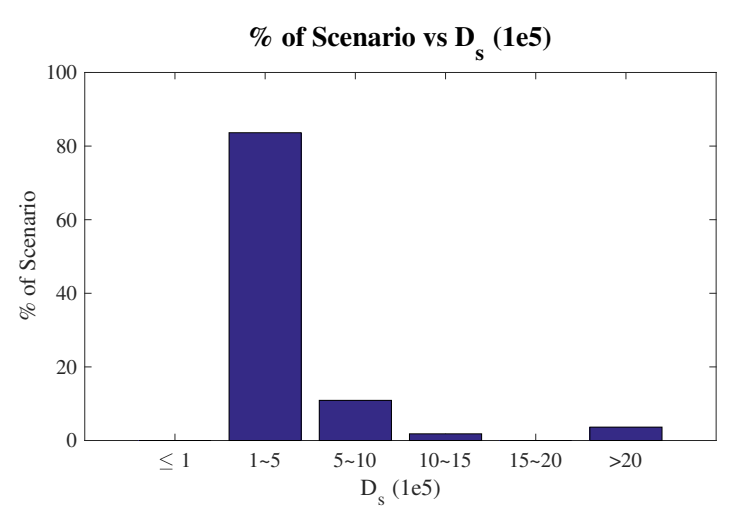

(a) The case of dynamic model

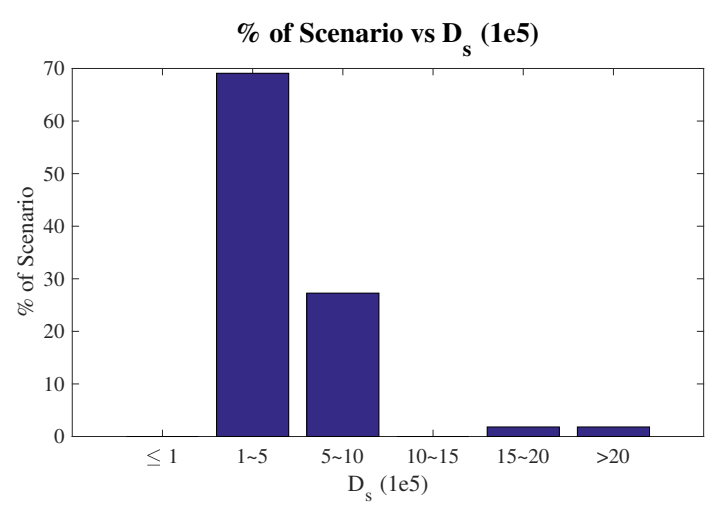

(b) The case of kinematic model

Figure 5.11: Total value of square of deviation

\subsection{Summary}

This chapter presented the use of finite horizon model predictive control for the case of a quad-rotor for real-time trajectory generation and autonomous obstacle avoidance while remaining close to a reference path. A comparable study between the problems with the kinematic and the dynamic model for a quad-rotor UAV is shown that includes the study with the change in $\Delta t$. From the fact that the problem with the kinematic model has fewer optimization variables compared to the dynamic model, the problem with the kinematic model takes less computation time, which is apparent from the Monte-Carlo test as well. From the study of $\Delta t$, and a numerical example for trajectory generation, it can be seen that the problems with the kinematic model and the dynamic model can have similar quality of trajectory with $\Delta t=300$ milliseconds. While from the MonteCarlo test, it is clear that the solution time for the problem with the kinematic model is significantly lower compared to the problem with the dynamic model. Although, the square of deviation test shows that the problem with the dynamic model performs slightly better than the kinematic counterpart, but the low solution time offered by the kinematic model is more attractive than the slight benefits in deviation with the dynamic counterpart. Therefore, in contrast to the case of a fixed-wing UAV, the quad-rotor UAV with the kinematic model performs reasonable in comparison to the quad-rotor dynamic model with the benefits of the solution time. 


\section{Chapter 6}

\section{Quad-rotor Swarm Application}

Chapter 5 studied trajectory generation for the case of a single quad-rotor using the model predictive control (MPC) framework and the custom solver. Results from Chapter 5 indicate that the kinematic model for a quad-rotor may be sufficient for trajectory generation whereas a trajectory can be generated using the dynamic model indirectly using the solution of kinematic model with the added advantage of significantly lower solution time. While a low solution time for a quad-rotor may help in a fast-paced environment, it also helps when quad-rotors are in a swarm. Thus, this chapter focuses on the use of the approach for a swarm of quad-rotors. In particular, a centralized approach is utilized, and dynamical, and control constraints are accommodated. However, computational complexity is one of the challenges that constrain the centralized approach for swarm scalability. Therefore, this chapter examines the computational aspect of swarm

problems. Two swarm problems are defined. The first problem is the same as the one studied in Chapter 5 but extended to a swarm of quad-rotors with the aim of studying the computational complexity for the problem with an increased number of UAVs for a swarm. The second problem has the same problem defining parameters as the first, besides it has a moving target point, where a target point is formed by the intersection of a reference path with a target plane. Apart from studying the computational complexity associated with this problem, it can represent complex scenarios where a moving target point may sometime be inside an obstacle. The second problem with the moving target point is solved in two ways, in an attempt to lower the solution time. In the first way, the optimal control problem in the model predictive control (MPC) framework is solved 
by considering the entire swarm at once. In the second way, it is solved iteratively for a UAV by considering trajectories from other UAVs as fixed. A numerical example shows that solving iteratively for a UAV at a time is almost four to seven times computationally faster than solving the swarm problem at once. However, this comes with the price that the found solution may not be completely optimal (may not be the same). In addition, using the same problem formulation a swarm problem with 32 UAVs were solved in 194.24 milliseconds in the laptop computer.

\subsection{Introduction}

In this chapter, an extended use of the custom solver from Chapter 2, and the kinematic model for a quad-rotor utilized in Chapter 5 to quad-rotor swarm application is presented. As it was observed, the computational advantage that the kinematic model exhibits over the dynamic counterpart in Chapter 5 , it is reasonable to expect that the swarm problem has the same benefit as well. However, the increase in the number of quad-rotors in a swarm increases the problem size which eventually makes the computability of the swarm problem questionable for real-time applications. Hence, to lower the computation time as needed in real-time trajectory generation for a swarm of quad-rotors a custom solver from Chapter 2 is used, that avoids unnecessary computations with zeros with the least possible memory requirement.

In this chapter, other ways are sought by which the problem could be solved to further reduce computation time. Specifically, a comparison is made between trajectories generated by a trajectory generation problem that solves for the entire swarm at once and considering a single agent at a time. In order to constrain from the broad topic of trajectory generation for a swarm of quad-rotors, only the method of trajectory generation using the central controller approach is investigated. The central controller is a central computational system that computes trajectory for all the UAVs in a swarm rather than the trajectories being generated locally onboard each UAV. One main assumption of using the central controller is the knowledge of the position of all the UAVs in a swarm. In this approach, the central controller generates collision-free trajectories for all the UAVs (quad-rotors) where the controller acts as a high level trajectory planner, and the respective UAV tracks the given trajectory. 
At first, a comparison is made between a problem size between the use of the kinematic and the dynamic model for a quad-rotor, where the models are introduced in Chapter 5. Then, a swarm problem is defined and solved which is an extension to the problem with the kinematic model in Chapter (5), but for a swarm of quad-rotors. The objective of solving the swarm problem is to understand the complexity and computability of the approach. Next, a swarm problem is defined which is the same as the previous swarm problem but with a slight different cost function. In this case, the cost function has moving targets for each UAVs. In the cost function defined in Chapters 4 and 5 , the term that helps UAVs progress along the desired direction of flight, along with a reference path can represet a point. Hence, it may sometimes be named as a target point. Thus, the target point is the same as the term used for advancing UAVs along the reference path in the desired direction of flight in Chapters 4 and 5 which are kept far enough and fixed such that a UAV may never reach there in finite time under the model predictive control (MPC) window, but in the current case the location of the target changes with time, hence the term moving (moving target) and a UAV may reach the target within the model predictive control (MPC) window. The reason for using the term to advance UAVs along the desired flight direction has been discussed in Section 4.2.3. In brief, the purpose of using the target point in the cost function is to drive/fly quad-rotors along the desired direction, rather than a hard constraint as the end-point constraint. One significant advantage of replacing a definitive goal point with the cost due to a target point is, even if the target point is inside an obstacle the problem still becomes end-point feasible, as such there is no hard constraint on target point. The problem serves applications that require a swarm of UAVs to progress along their flight direction with certain progression in the desired direction of flight, where the speed of moving targets can dictate the progress in the direction of flight for the swarm. Such a scenario could arise in an aerial survey that uses a swarm of UAVs in obstacle-rich environment where each UAVs in the swarm may have different progression requirement. Also, a scenario such as a swarm chasing suspects in city like environment fits into the problem formulation. Finally, the above swarm problem with the moving targets is solved in a different way, such that the swarm problem is solved iteratively for a UAV in a model predictive control (MPC) time instant considering the trajectories from other UAVs as fixed.

The framework that is used in this Chapter to solve the problems is the finite horizon 
model predictive control (MPC) approach as outlined in Section 4.3, with the same constraints and the cost function as the original problems. Even though, there are moving targets in the problems, it is not a hard constraint but a factor that drives UAVs along its direction by minimizing the distance between UAVs and target points as cost function, as used in the problems of Chapter 4 and Chapter 5. In order to solve the model predictive control (MPC) problem the sequential convex programming (SCP) approach as outlined in Section 2.2 is used, where the maximum number of sequential convex programming (SCP) iterations is limited to five. The sequential convex programming (SCP) approach outlined solves sequences of quadratic programming (QP) sub-problem within it, for which a custom solver from Chapter 2 is used. The problems will be computed in Matlab environment using MEX-file in a laptop computer with $1.8 \mathrm{GHz}$ processor and 4 GB RAM.

The contents in this chapter are organized as follows. Section 6.2 introduces a swarm problem and compares the problem size between the use of the kinematic and the dynamic model, where models for a quad-rotor are introduced in Chapter 5. The swarm problem which is an extension to the problem with kinematic model in Chapter 5 is then defined and a numerical example is presented in Section 6.3. In Section 6.4 a swarm problem is defined which is the same as the problem in Section 6.3 but with a slight different cost function. It has moving targets, a numerical example is presented. Then, in Section 6.5 the problem in Section 6.4 is solved with different approach, a numerical example, Monte-Carlo study and scalability study are presented. Finally, Section 6.6 summarizes the chapter.

\subsection{Problem Size for a Swarm}

Let us define a swarm problem as similar to (5.24) in order to study its problem size. Let there be $N_{s}$ number of quad-rotor UAVs in a swarm, and suppose $\dot{X}^{j}=f\left(X^{j}, U^{j}\right)$ represents a system model as similar to (5.4) for the $j^{\text {th }}$ quad-rotor, and $J(X(\cdot), U(\cdot))$ represents a performance index as similar to (5.22) but for a swarm, where $X=\left[X^{1 T}, \ldots, X^{N_{s} T}\right]^{T}$, $U=\left[U^{1 T}, \ldots, U^{N_{s} T}\right]^{T}$, and $j=1,2, \ldots, N_{s}$. Let us define an initial point for the $j^{\text {th }}$ quadrotor to be $X_{0}^{j}=\left(x_{0}^{j}, y_{0}^{j}, z_{0}^{j}\right)$, while the aim of each quad-rotor is to fly along a desired direction of flight while remaining close to a reference path. Let the collision avoidance 
constraint among the UAVs in a swarm be as in [24],

$$
\left\|\left[\begin{array}{c}
x^{k}-x^{j} \\
y^{k}-y^{j} \\
z^{k}-z^{j}
\end{array}\right]\right\| \geq R_{C A}, \forall k \neq j, k, j \in\left\{1, \ldots, N_{s}\right\}
$$

where $k$ and $j$ refer to two different UAVs in a swarm, and $R_{C A}$ is the radius of collision avoidance which also represents the centre distance between two UAVs.

Now a trajectory generation problem for a swarm of quad-rotors is defined as, given the initial conditions for each UAV in the swarm at time $\tau_{0}$, find the state-control pair $(X(t), U(t))$ to minimize the cost $J(X(\cdot), U(\cdot))$ subject to,

$$
\begin{gathered}
\dot{X}^{j}=f\left(X^{j}, U^{j}\right) \\
h_{i}^{j}(x, y, z)=\ln \left[\left|\left(\frac{x^{j}-x_{\{c, i\}}}{a_{i}}\right)^{p_{x_{i}}}\right|+\left|\left(\frac{y^{j}-y_{\{c, i\}}}{b_{i}}\right)^{p_{y_{i}}}\right|+\left|\left(\frac{z^{j}-z_{\{c, i\}}}{c_{i}}\right)^{p_{z_{i}}}\right|\right] \geq \epsilon>0 \\
\left\|\left[x^{k}-x^{j}, y^{k}-y^{j}, z^{k}-z^{j}\right]\right\| \geq R_{C A}, \forall k \neq j \\
T^{j} \leq T_{\max } \\
X=\left[X^{1 T}, \ldots, X^{N_{s} T}\right]^{T} \\
U=\left[U^{1 T}, \ldots, U^{N_{s} T}\right]^{T} \\
j, k=1, \ldots, N_{s}
\end{gathered}
$$

where $h_{i}^{j}(x, y, z)$ represents the obstacle avoidance constraint for each quad-rotor $j$ with each obstacle $i$, and $T^{j} \leq T_{\max }$ represents the total thrust limitation for a quad-rotor $j$ as in (5.24).

\subsubsection{Problem Size Comparison}

In Chapter 5 it was found that a trajectory generation problem with the kinematic model for a quad-rotor has fewer optimization variables compared to a trajectory generation problem with the dynamic model. However, the issue is examined for a swarm of quadrotors. Although a ground based computer could have significant greater computational capability compared to an on-board computer, for real-time control of a swarm of quadrotors, computational demands cannot be overlooked, and the ground based computer may also be restricted to the capability to compute the trajectory in real-time. As the 
number of quad-rotors in a swarm increase, the constraints and optimization variables increase also. This increases the computational burden to solve for a swarm problem.

Let us consider the swarm problem (6.2), where the problem is solved using a finite horizon approach within Model Predictive Control framework as in Chapter 5. Let us suppose there are $N_{s}$ number of UAVs in a swarm with $S$ number of states and $C$ number of controls per UAV. Let us consider that the swarm problem is discretized to $N$ number of nodes and that there are $O$ number of obstacles. Then, expressions are derived that give the number of optimization variables and constraints for the swarm problem in the following.

Since the problem (6.2) will be solved within the model predictive control (MPC) framework (from Section 4.3) and the problem has $N$ discrete horizon, it will have $N-1$ control horizon. Thus, the problem will have $N_{s} N S$ number of optimization variables related to the state of UAVs whereas, $N_{s}(N-1) C$ number of optimization variables related to the control of UAVs. Therefore, the total number of optimization variables for the problem wil be,

$$
\text { Number of Optimization variables }=N_{s}(S N+C(N-1))
$$

Likewise, the problem will have equality constraints from the UAVs system equation which will sum up to $N_{s} S N$ for $N_{s}$ number of UAVs with $S$ number of states in $N$ number of nodes. Since, the inequality constraint due to obstacle are for all the UAVs in the swarm, thus it results in $N_{s} O N$ number of constraints. The collision avoidance constraint for UAVs results in $N N_{s}\left(N_{s}-1\right) / 2$ constraints as a UAV requires avoidance with every other UAVs. The inequality on the thrust results in $N N_{s}$ constraints. Finally, there are box constraints (the lower and the upper bounds) for optimization variables which result in $2\left(N_{s} N S+N_{s}(N-1) C\right)$ constraints. Therefore, altogether the number of constraints will be,

$$
\text { Number of Constraints }=N_{s}\left[N\left(3 S+O+1+\frac{N_{s}-1}{2}\right)+2(N-1) C\right]
$$

In the following a comparison is made between the size of the swarm problem (6.2), between the use of the UAV model (5.3) and the kinematic model (5.4). 


\section{A Swarm Problem with the Dynamic Model}

For the problem with the UAV model (5.3), the number of states is $S=10$ and the number of controls $C=4$. Let there be 7 obstacles, $O=7$ and the number of nodes used for the problem be $N=20$. Table 6.1 shows the size of the swarm problem with increasing number of UAVs $\left(N_{s}\right)$ in a swarm, where the number of optimization variables are calculated from (6.3), and the number of constraints are computed from (6.4).

Table 6.1: Size of the swarm problem using the UAV model (5.3)

\begin{tabular}{ccc}
\hline \hline$N_{s}$ & No. of Optimization Variables & No. of Constraints \\
\hline 1 & 276 & 912 \\
6 & 1656 & 5772 \\
16 & 4416 & 16992 \\
32 & 8832 & 39104 \\
64 & 17664 & 98688 \\
128 & 35328 & 279296 \\
256 & 70656 & 886272 \\
\hline \hline
\end{tabular}

\section{A Swarm Problem with the Kinematic Model}

Similarly, for the problem with the kinematic model (5.4), the number of states is $S=6$ and the number of control is $C=3$. The same number of obstacle and the number of node $N$ are used, as above. Table 6.2 presents the size of the swarm problem with different number of UAVs $\left(N_{s}\right)$ in the swarm.

Table 6.2: Size of the swarm problem using the UAV model (5.4)

\begin{tabular}{ccc}
\hline \hline$N_{s}$ & No. of Optimization Variables & No. of Constraints \\
\hline 1 & 177 & 634 \\
6 & 1062 & 4104 \\
16 & 2832 & 12544 \\
32 & 5664 & 30208 \\
64 & 11328 & 80896 \\
128 & 22656 & 243712 \\
256 & 45312 & 815104 \\
\hline \hline
\end{tabular}

Figure 6.1 shows the visual comparison of the number of optimization variables, and 
the number of constraints, in the swarm problem from table 6.1 and 6.2. From the figure, it can be seen that the kinematic model offers less computational complexity for the swarm problem with increase in a swarm size compared to the use of the dynamic model. Hence, for a swarm application of quad-rotors the kinematic model is used.

The number of variables and constraints comparison

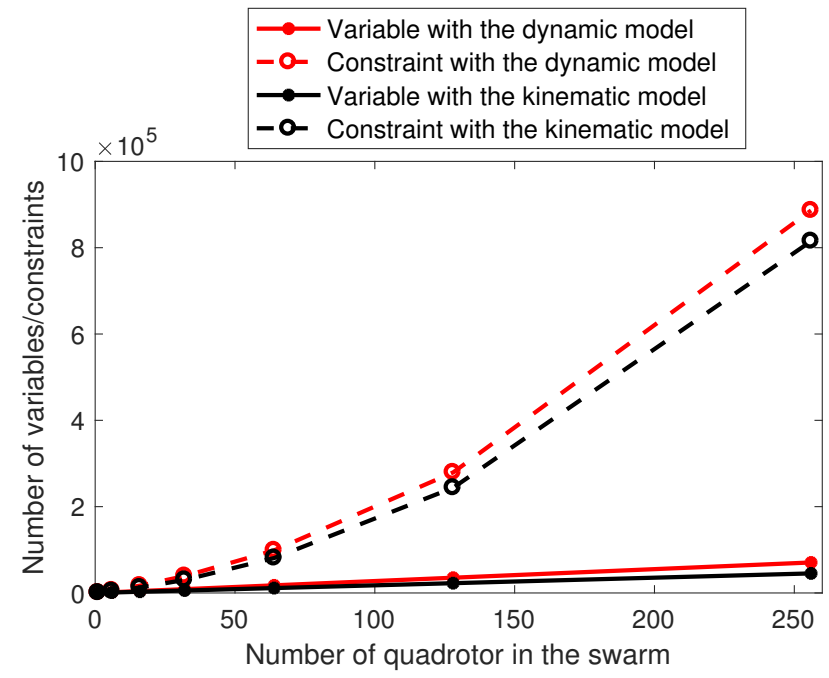

Figure 6.1: Problem size comparison between the kinematic and the dynamic model

\subsection{Swarm Problem with Reference Paths}

Consistent with the scenarios considered in Chapters 3 and 4, a swarm problem is considered where the swarm is required to fly along a desired flight path, without necessarily having a final goal point. The problems considered in Chapter 5 are problems without a goal point, even though they have a term that drives a UAV along the desired direction. Thus, in this section the Problem (5.24) is extended to a swarm application. Figure 6.2 shows the representation where three quad-rotors have their respective reference paths for each of them to fly along in the desired direction. The quad-rotors while flying close to their reference path have to avoid obstacles which may be a stationary or moving, and also avoid collision among each other. 


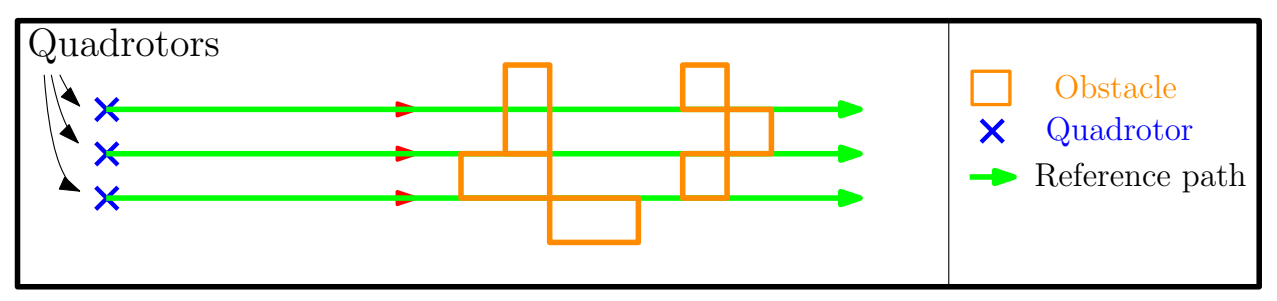

Figure 6.2: Swarm problem 2

\subsubsection{Problem Definition}

Let us suppose that there are $N_{s}$ number of quad-rotors in a swarm. Let $y^{j}(t)$ and $z^{j}(t)$ represent the position of a $j^{\text {th }}$ quad-rotor along $y$ and $z$ axes at time $t$. As in Chapter 4 and 5, without loss of generality it is assumed that the desired flight direction is parallel to the $\mathrm{x}$-axis, with $\mathrm{y}$ and $\mathrm{z}$ coordinates given by constant $y_{d}^{j}$ and $z_{d}^{j}$ respectively for the $j^{\text {th }}$ quad-rotor. The $x_{a}^{j}$ is chosen large enough such that a quad-rotor $j$ can never reach the point $x_{a}^{j}$ at time $\tau_{f}$. In addition, the robustness function is added for UAVs in the swarm associated with obstacles. Furthermore, to minimize the chances of jerky controls, the square of the rate of change of controls is added to the running cost as $\left(\dot{U}^{j}(t)\right)^{2}$ (In Chapter 5, to perform a comparative study between the models which have different control variables between them, such cost was not added.) Thus, the cost function for the trajectory generation problem for a swarm of quad-rotors to avoid obstacles and remain close to the reference path while flying from $\left(x_{0}^{j}, y_{0}^{j}, z_{0}^{j}\right)$ at time $\tau_{0}$, up to final time $\tau_{f}$ can be written as,

$$
\begin{gathered}
J(X(\cdot), U(\cdot))=\sum_{j=1}^{N_{s}} \int_{\tau_{0}}^{\tau_{f}}\left[\alpha\left(y^{j}(t)-y_{d}^{j}\right)^{2}+\beta\left(z^{j}(t)-z_{d}^{j}\right)^{2}+\eta\left(x^{j}(t)-x_{a}^{j}\right)^{2}\right. \\
\left.+\bar{r}\left(X^{j}(\cdot)\right)+\zeta\left[\dot{U}^{j}(t)\right]^{T}\left[\dot{U}^{j}(t)\right]\right] d t
\end{gathered}
$$

where $\zeta>0$ is the penalty term associated with the square of the rate of change of controls, besides that all the terms are as stated in Section 4.2.3 .

Since, all the problem defining parameters have already been discussed in Section 6.2 of this chapter, the problem is defined as, 


\section{Problem SP1:}

Given an initial condition $\left(x_{0}^{j}, y_{0}^{j}, z_{0}^{j}\right)$ for a $j^{\text {th }}$ quad-rotor at time $\tau_{0}$, find the control-state pair $(X(\cdot), U(\cdot))$ to minimize the cost (6.5) subject to

$$
\begin{aligned}
& \text { UAV model (5.4) as, } \quad \dot{X}^{j}=f\left(X^{j}, U^{j}\right) \\
& \text { Obstacle constraint in (6.2) as, } \quad h_{i}^{j}(x, y, z) \geq \epsilon, \forall i=1, \ldots, O \\
& \text { Collision avoidance constraint (6.1) } \\
& \text { Thrust constraint ( } T \text { as (5.14)), } \quad T^{j} \leq T_{\max } \\
& \qquad \begin{array}{c}
X=\left[X^{1 T}, \ldots, X^{N_{s} T}\right]^{T} \\
U=\left[U^{1 T}, \ldots, U^{N_{s} T}\right]^{T} \\
\\
\qquad, k=1, \ldots, N_{s}
\end{array}
\end{aligned}
$$

As similar to the problems in Chapters 4 and 5 the swarm problem is solved using the finite horizon model predictive control (MPC) approach as outlined in Section 4.3, with the same constraints and the cost function as the original problem, where the problem is discretized at the sampling time up to the finite horizon. Then, the discretized problem is solved using sequential convex programming (SCP) method as presented in Section 2.2 where a custom solver from Chapter 2 is used to solve a sub-problem within it.

\subsubsection{Numerical Example}

As an example, a scenario is defined where a swarm of six quad-rotors flies along with their reference paths along the positive $x$ - axis. The starting point for each quad-rotor in the swarm, along with their respective reference path is given in Table 6.3. All other problem defining parameters for this example are as given in Table 5.1 where the sampling time of 300 milliseconds is used, and the point $x_{a}$ for all the quad-rotors have been set to 240 meters as shown in the table. The penalty term $\zeta$ associated with the rate of change of controls from (6.5) has been set to 0.2. The obstacles under consideration for this example case are given in Appendix 4.5. The parameters for each quad-rotor are taken from Appendix 4.1, and the collision avoidance distance, $R_{C A}$, among quad-rotors has been set to 20 meters.

Figure 6.3 shows the complete trajectory for the swarm with drones starting from their respective initial points. The quad-rotors are arranged at the distance of 100 meters along 
Table 6.3: Starting points and desired paths for quad-rotors

\begin{tabular}{cccc}
\hline \hline Quad-rotor $(j)$ & Initial Point $\left(x_{0}^{j}, y_{0}^{j}, z_{0}^{j}\right) m$ & $y_{d}^{j} m$ & $z_{d}^{j} m$ \\
\hline 1 & $(0,50,20)$ & 50 & 20 \\
2 & $(0,50,60)$ & 50 & 60 \\
3 & $(0,150,20)$ & 150 & 20 \\
4 & $(0,150,60)$ & 150 & 60 \\
5 & $(0,250,20)$ & 250 & 20 \\
6 & $(0,250,60)$ & 250 & 60 \\
\hline \hline
\end{tabular}

the $y$-axis and 40 meters along the $z$-axis. As the collision avoidance distance among quad-rotors have been set to $R_{C A}=20$ meters, the trajectories of the quad-rotors at the height of 60 meters are completely unaffected by all UAVs, and also the obstacles at the lower height which we can see in Figure 6.5.

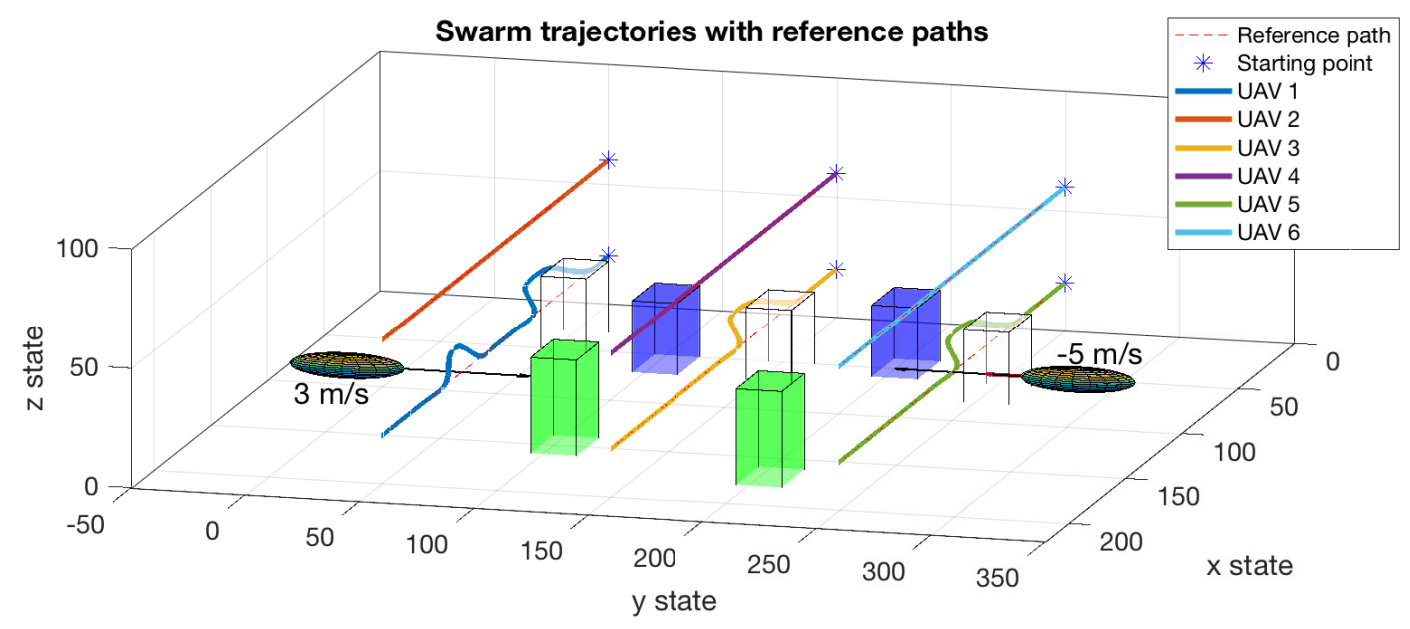

Figure 6.3: 3D view for the swarm example

In Figure 6.4 it can be seen that the UAVs at the lower heights avoid the obstacles safely. One of the moving obstacles that start at 300 meters along the $y$-axis does not have an impact on the trajectory of quad-rotors at the lower heights as the obstacle passes the intersection point early for the UAV 5, and later for the UAV 3 and UAV 1. However, the second moving obstacle that starts at 0 meter along the $y$ - axis impacts the trajectory of UAV 1 and does not alter the trajectories of other UAVs.

In Figure 6.6, trajectories of UAVs at time $t=25$ seconds can be seen, where UAV 1 moves along $z$-axis to avoid the moving obstacle, and Figure 6.7 shows the top view of 


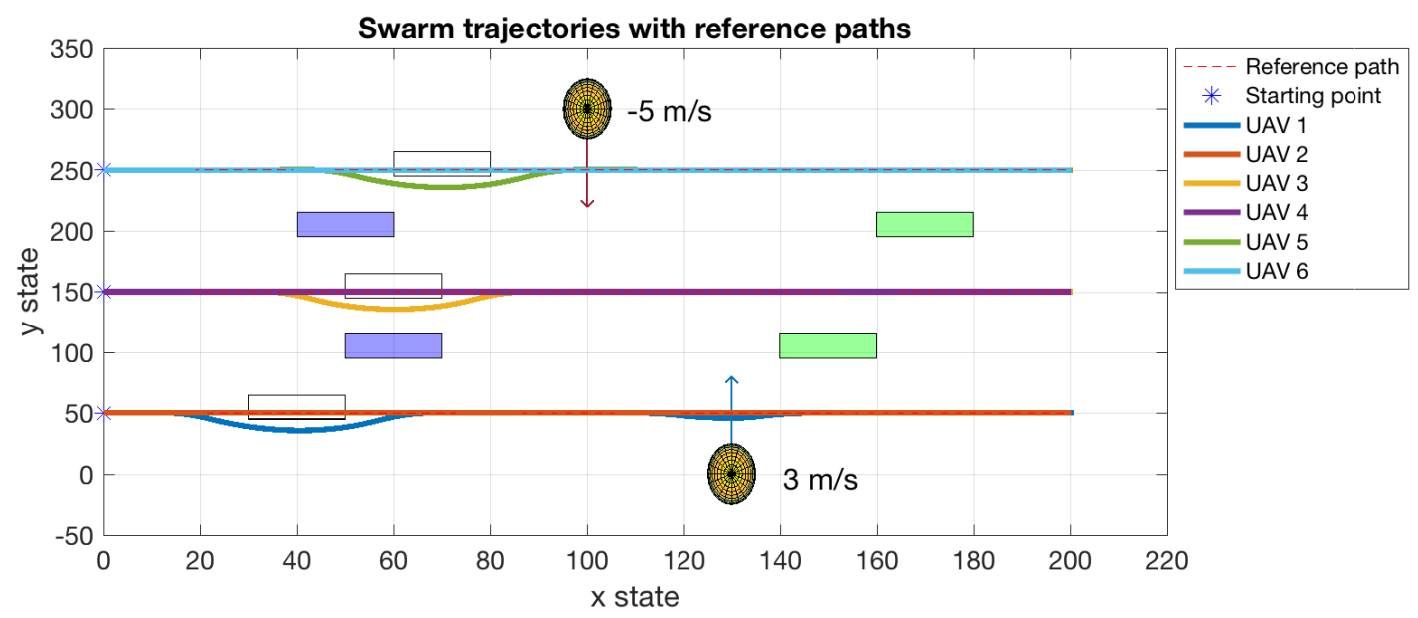

Figure 6.4: XY view for the swarm example

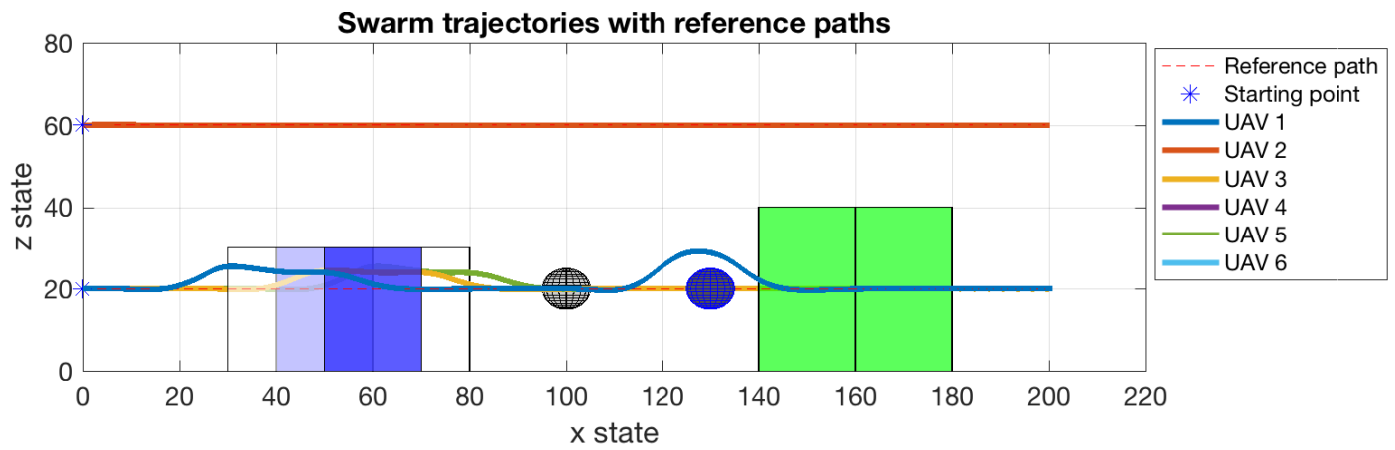

Figure 6.5: XZ view for the swarm example

the scenario at time $t=26$ seconds where the UAV comes close to the moving obstacle but performs an avoidance maneuver by slightly deviating from the reference path. The reason $t=25$ and $t=26$ seconds are shown is because of a visual clarity in the avoidance maneuver. From both the figures, it can be seen that UAV 3 has already passed the intersection point with the obstacle coming towards it from $y=300$ meters. Similarly, UAV 1 flies above the moving obstacle going towards the positive $y$-axis in order to avoid the collision.

Figure 6.8 and 6.9 shows the location of UAVs at time $t=40$ seconds with $x-y$ and $x-z$ view respectively. It is almost the same as figures 6.4 and 6.5 , except that all the UAVs progress the same distance along $x$ - axis despite obstacle avoidance.

Figure 6.10 shows the computation time for solving the swarm problem, at each model 


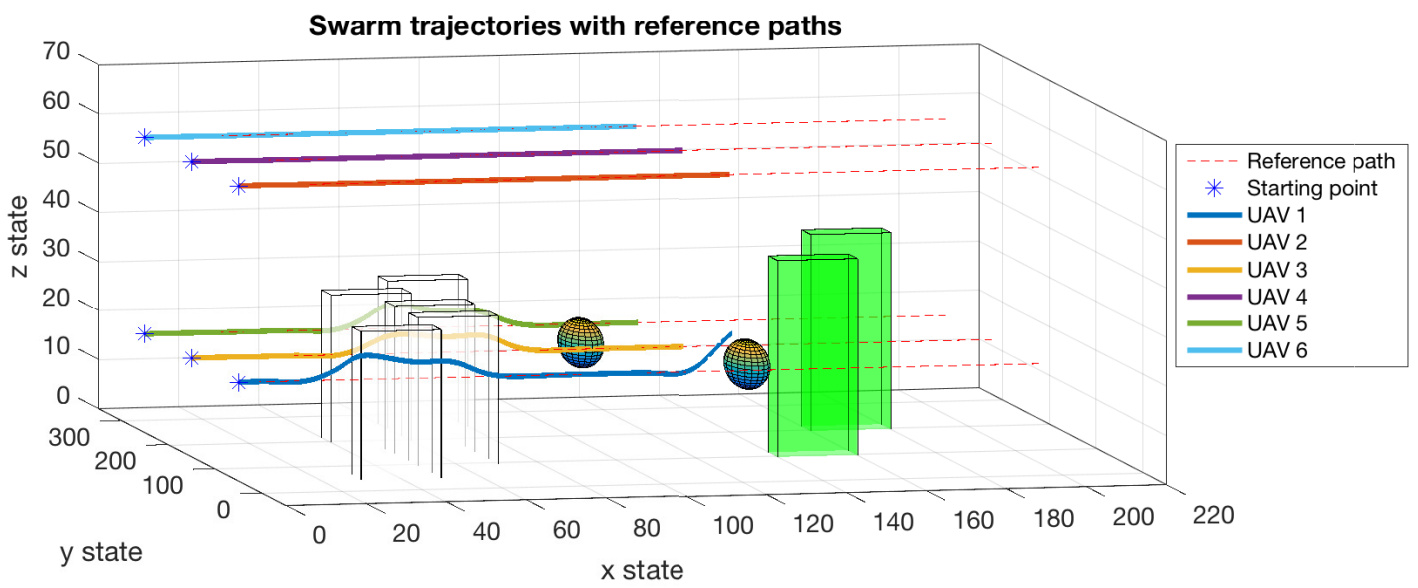

Figure 6.6: The swarm scenario at $t=25$ seconds

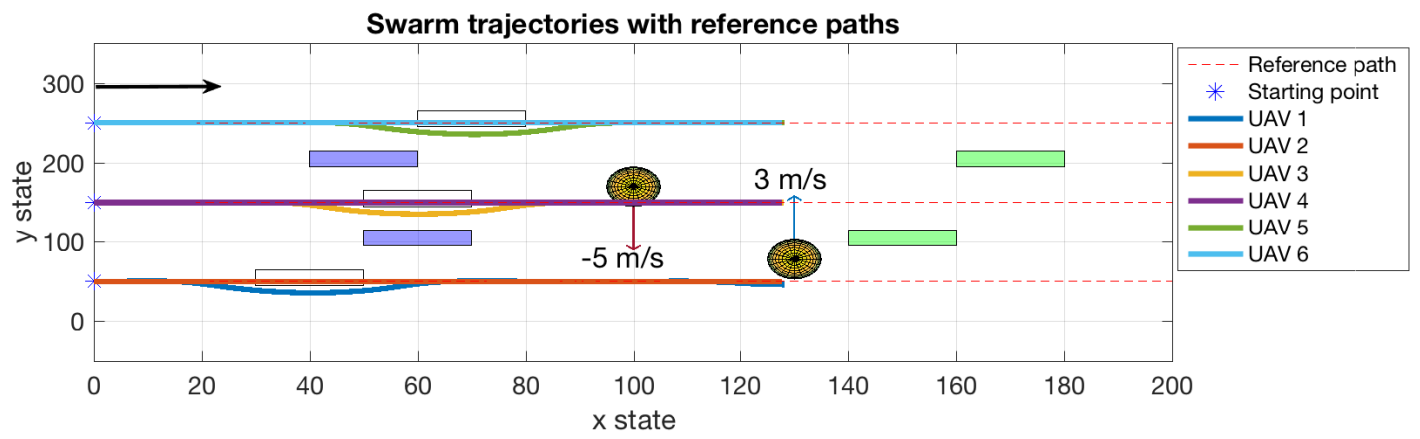

Figure 6.7: The swarm scenario at $t=26$ seconds

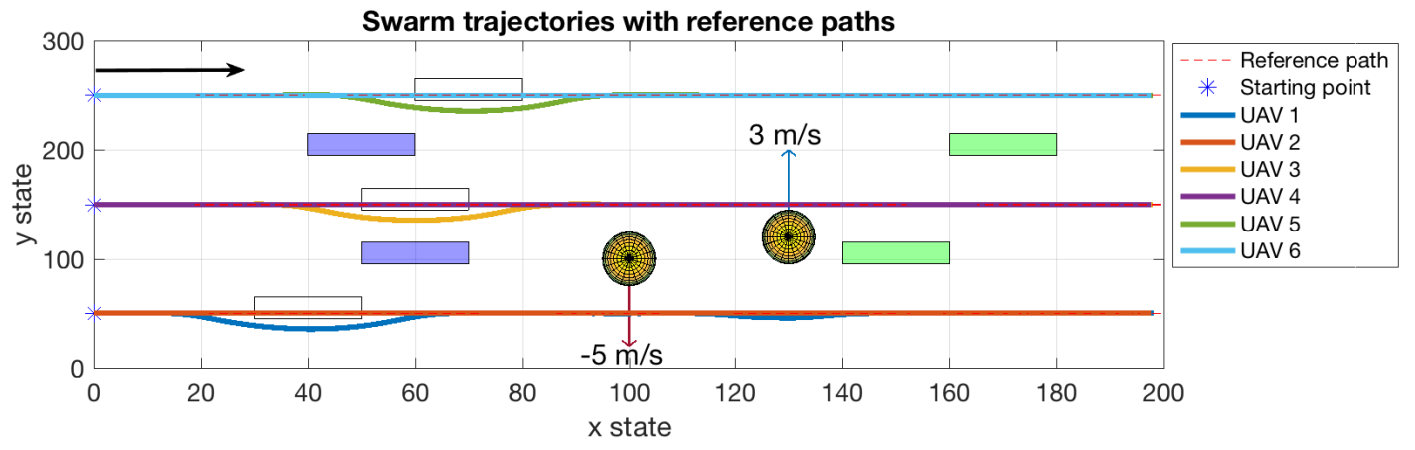

Figure 6.8: XY view of the swarm scenario at $t=39.9$ seconds

predictive control (MPC) time instant $t$ using the custom solver from Chapter 2. It takes 40.2 seconds of flight time for the swarm to reach the point $x=200$ meters, where the 


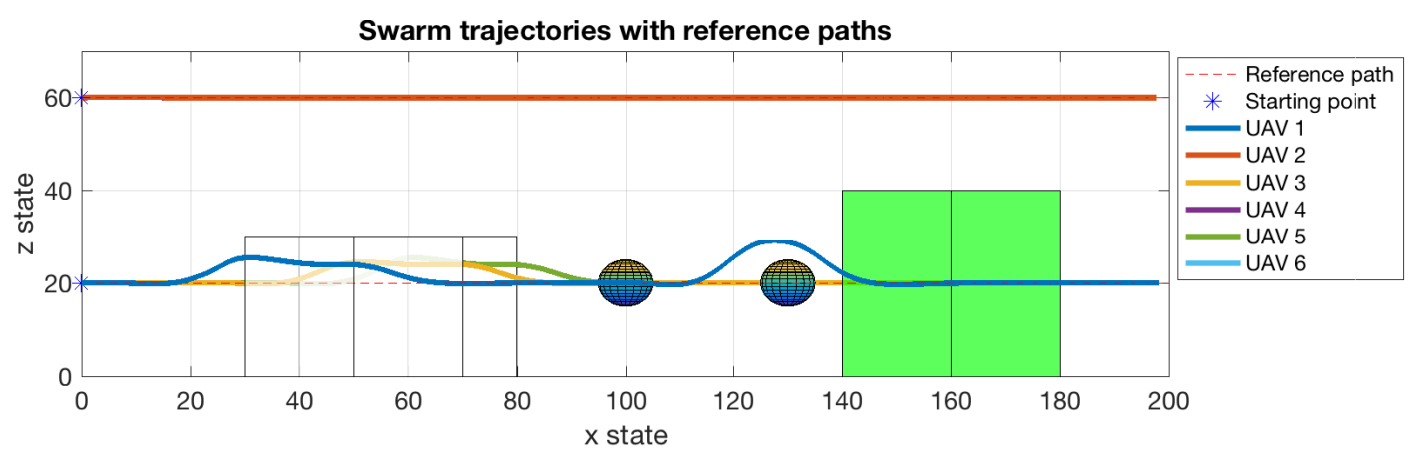

Figure 6.9: XZ view of the swarm scenario at $t=39.9$ seconds

simulation is stopped. The maximum solution time for the swarm is 181.24 milliseconds, while the minimum solution time is 93.26 milliseconds. The solution time reflects the amount of computational complexity. The problem has 36570 nonzero entries in the Karush-Kuhn-Tucker (KKT) matrix of its quadratic programming (QP) sub-problem. In addition, the figure compares the solution time between the problem with a quadrotor UAV in Chapter 5 which is (5.24), and the swarm problem of this section which is SP1. The comparison shows that with just the 6 UAVs the solution time to the swarm problem grows significantly towards the control horizon $(\Delta t)$. If we are to compare between the average solution time in between these problems then the average solution time for Problem (5.24) is 2.9561 milliseconds shown by black dashed line, while for Problem SP1 an average solution time is 118.3161 milliseconds shown by red dashed line.

\section{Comparison with ECOS and IPOPT}

Similar to Section 5.3.2, the trajectory generation in the above scenario using CS is compared to with ECOS and IPOPT in terms of cost and computation time. While there are six quad-rotors in the swarm with six different reference path, the cost of all of the UAVs with respect to their respective reference paths are added together to signify as total cost value. In the comparison, CS has the least value of cost with the least computation time followed by IPOPT with approximately similar cost but with the moderate maximum computation time (compared to with ECOS). In this case, ECOS is not only expensive in terms of the value of cost, but also has the highest computation time. 


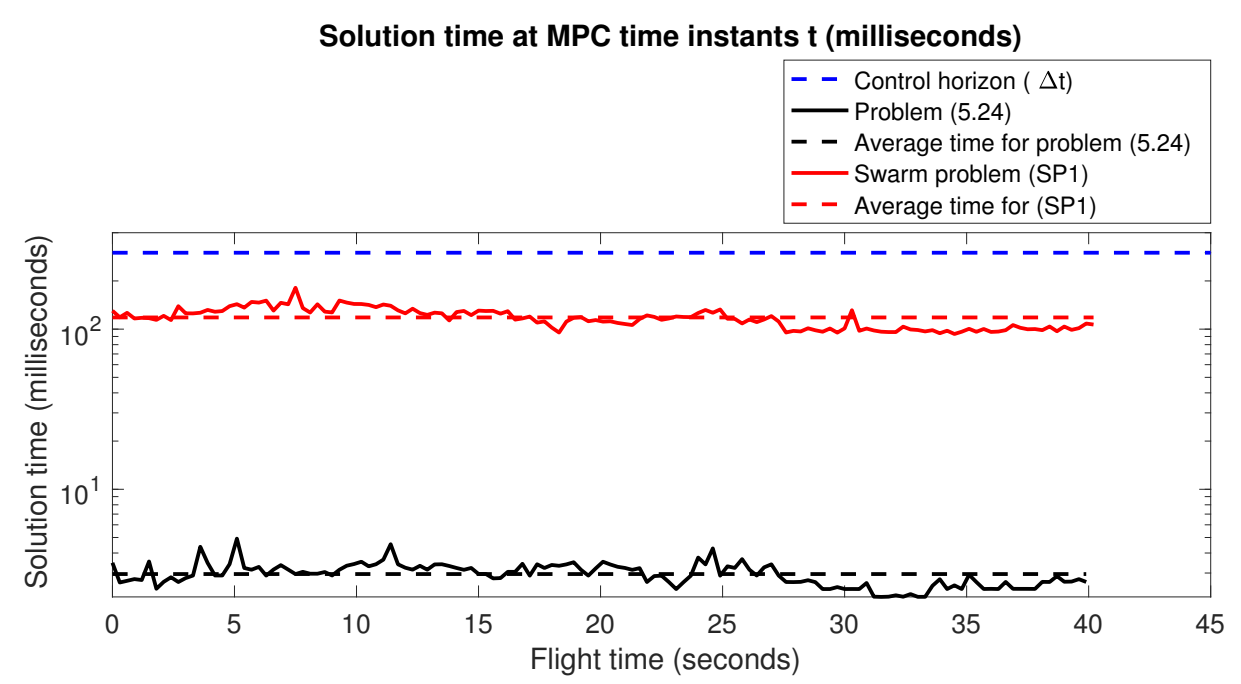

Figure 6.10: Solution time for the swarm problem at time $t$

Table 6.4: Comparison of custom solver (CS) with IPOPT and ECOS.

\begin{tabular}{cccc}
\hline \hline & Cost & Average computation time $(\mathrm{ms})$ & Maximum computation time (ms) \\
\hline IPOPT & 16.05 & 642.0 & 949.9 \\
\hline ECOS & 57.90 & 889.5 & 3916.9 \\
\hline CS & 16.04 & 118.3 & 181.2 \\
\hline \hline
\end{tabular}

\subsection{Swarm Problem with Moving Targets}

The trajectory generation problem that is considered next is the one with moving targets. As stated in the introduction, the target points are merely the terms in the cost function that help UAVs to advance along the desired direction. Figure 6.11 shows a representation for this type of scenario where two quad-rotors aim to reach their respective moving targets while flying along their reference paths in the desired direction of flight, while avoiding obstacles and collision among themselves. Thus, given initial points (starting points) for a swarm of quad-rotor the focus is on the trajectory generation problem where each quad-rotor has a reference path to remain close to while all the quad-rotors in the swarm aim to reach their respective moving targets by avoiding collision among themselves and maneuvering around obstacles with robustness, and satisfying states and controls constraints. 


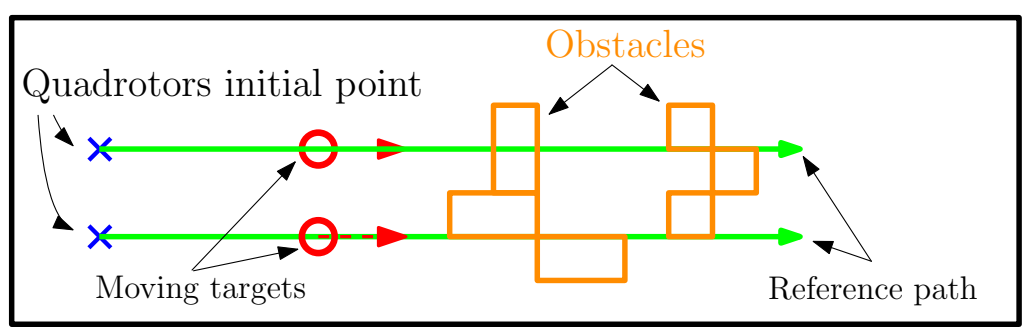

Figure 6.11: Swarm problem with moving targets

\subsubsection{Problem Definition}

The problem definition for this case differs from Problem SP1 only in the cost function, besides that, all the constraints are the same. Let us define

$$
X_{m}^{j}(t)=\left[x(t)_{m}^{j}, \dot{x}(t)_{m}^{j}, y(t)_{m}^{j}, \dot{y}(t)_{m}^{j}, z(t)_{m}^{j}, \dot{z}(t)_{m}^{j}\right]^{T} \text { as the state of the moving }
$$
target at time $t$ associated with a quad-rotor $j$. Then, the cost function is written for the trajectory generation problem for a swarm of quad-rotors with moving targets to avoid obstacles and remain close to their respective reference path while flying from $\left(x_{0}^{j}, y_{0}^{j}, z_{0}^{j}\right)$ at time $\tau_{0}$, up to final time $\tau_{f}$ as,

$$
J(X(\cdot), U(\cdot))=\sum_{j=1}^{N_{s}}\left[\int_{\tau_{0}}^{\tau_{f}} F\left(X^{j}(t), U^{j}(t), t\right) d t\right]
$$

where

$$
F\left(X^{j}(t), U^{j}(t), t\right)=\left(\begin{array}{c}
\alpha\left(y^{j}(t)-y_{d}^{j}\right)^{2}+\beta\left(z^{j}(t)-z_{d}^{j}\right)^{2}+ \\
\eta\left[X^{j}(t)-X_{m}^{j}(t)\right]^{T}\left[X^{j}(t)-X_{m}^{j}(t)\right]+w\left(\bar{r}\left(X^{j}(\cdot)\right)\right)+ \\
\zeta\left[\dot{U}^{j}(t)\right]^{T}\left[\dot{U}^{j}(t)\right]
\end{array}\right)
$$

Besides all the terms that have been defined in Chapter 4 and $5, \eta>0$ is associated with the cost that minimizes the square of difference between moving targets and UAVs, and $\zeta>0$ is associated with the cost that minimizes the square of the rate of change of controls.

The trajectory generation problem for a swarm with moving targets considered is, 


\section{Problem SP2-a:}

Given an initial condition $\left(x_{0}^{j}, y_{0}^{j}, z_{0}^{j}\right)$ for a $j^{\text {th }}$ quad-rotor at time $\tau_{0}$, find the control-state pair $(X(t), U(t))$ to minimize the cost (6.6) subject to

$$
\begin{aligned}
& \text { UAV model (5.4) as, } \quad \dot{X}^{j}=f\left(X^{j}, U^{j}\right) \\
& \text { Obstacle constraint in (6.2) as, } \quad h_{i}^{j}(x, y, z) \geq \epsilon, \forall i=1, \ldots, O \\
& \text { Collision avoidance constraint (6.1) } \\
& \text { Thrust constraint }\left(T \text { as (5.14)), } \quad T^{j} \leq T_{\max }\right. \\
& \qquad \begin{array}{c}
X=\left[X^{1 T}, \ldots, X^{N_{s} T}\right]^{T} \\
U=\left[U^{1 T}, \ldots, U^{N_{s} T}\right]^{T} \\
\\
\qquad, k=1, \ldots, N_{s}
\end{array}
\end{aligned}
$$

As stated in the introduction, to solve the swarm problem SP2-a the model predictive control (MPC) framework is utilized, where at each model predictive control (MPC) time instant a problem is initialized the problem as outlined in 4.3, and use sequential convex programming (SCP) approach from Chapter 2 to solve it.

\subsubsection{Numerical Example}

An example with six quad-rotors are considered in a swarm that fly along their respective reference paths in the positive $x$ - axis, while the aim is to reach a moving target per UAV (for all the quad-rotors). The starting points for each quad-rotor in the swarm, along with their respective reference paths are given in Table 6.5. In this example UAV 1 and UAV 2 start 20 meters apart (similarly for UAVs 3 and 4, and UAVs 5 and 6) therefore the collision avoidance distance, RCA, cannot be exactly 20 meters so as to avoid infeasible problem at the start (for numerical solving). Thus, the RCA has been set to 19 meters between all the UAVs.

The parameters for this example are taken from Table 5.1 with the sampling time of 300 milliseconds. The penalty term $\zeta$ associated with the rate of change of controls from (6.7) has been set to 0.2. The list of obstacles for this scenario is given in Appendix 4.5. The parameters for each quad-rotor are taken from Appendix 4.1. The moving targets for each UAV start on the reference paths at $x=20$ meters and moves along the positive $x$-axis on reference paths with the velocity vector of $(3,0,0) \mathrm{m} / \mathrm{s}$. As stated 
in the introduction the swarm scenario is solved in the model predictive control (MPC) framwork outlined in Section 4.3, therefore the initilization method is the same, as used in Chapters 4 and 5. So as to terminate the trajectory generation, the simulation is stopped once the moving target point reaches $x=300$ meters.

Table 6.5: Starting points for quad-rotors (units in meter)

\begin{tabular}{cccc}
\hline \hline Quad-rotor $(j)$ & Initial Point $\left(x_{0}^{j}, y_{0}^{j}, z_{0}^{j}\right)$ & $y_{d}^{j}$ & $z_{d}^{j}$ \\
\hline 1 & $(0,100,20)$ & 100 & 20 \\
2 & $(0,100,40)$ & 100 & 40 \\
3 & $(0,150,20)$ & 150 & 20 \\
4 & $(0,150,40)$ & 150 & 40 \\
5 & $(0,200,20)$ & 200 & 20 \\
6 & $(0,200,40)$ & 200 & 40 \\
\hline \hline
\end{tabular}

Figure 6.12 shows the complete trajectories for the example problem. In the figure two moving obstacles are shown at their respective initial locations. In among the moving obstacles, the one that goes from $y=0$ meters along positive $y$-axis causes UAV 1 to perform maneuver about its reference trajectory, and this obstacle as well affect UAV 3 at the same height ( $z=20$ meters), however UAV 5 at the same height already crosses the interception line with the obstacle. The moving obstacle that comes from $y=300$ meters causes all only the UAV 3 at the height of 20 meters to perform avoidance maneuver.

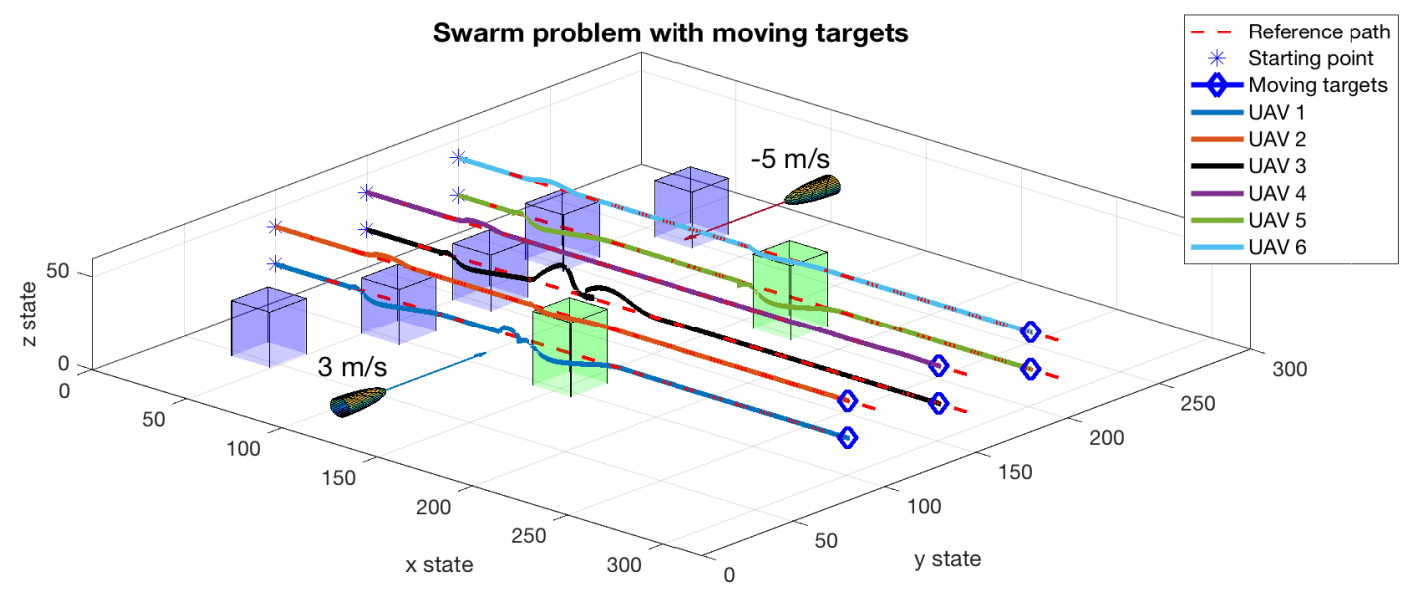

Figure 6.12: 3D view of the swarm with moving targets

Figure 6.13 shows the XY view of Figure 6.12, in which moving obstacles are shown 
at their respective initial positions. The figure shows that the trajectories remain close to their reference paths once obstacles are avoided.

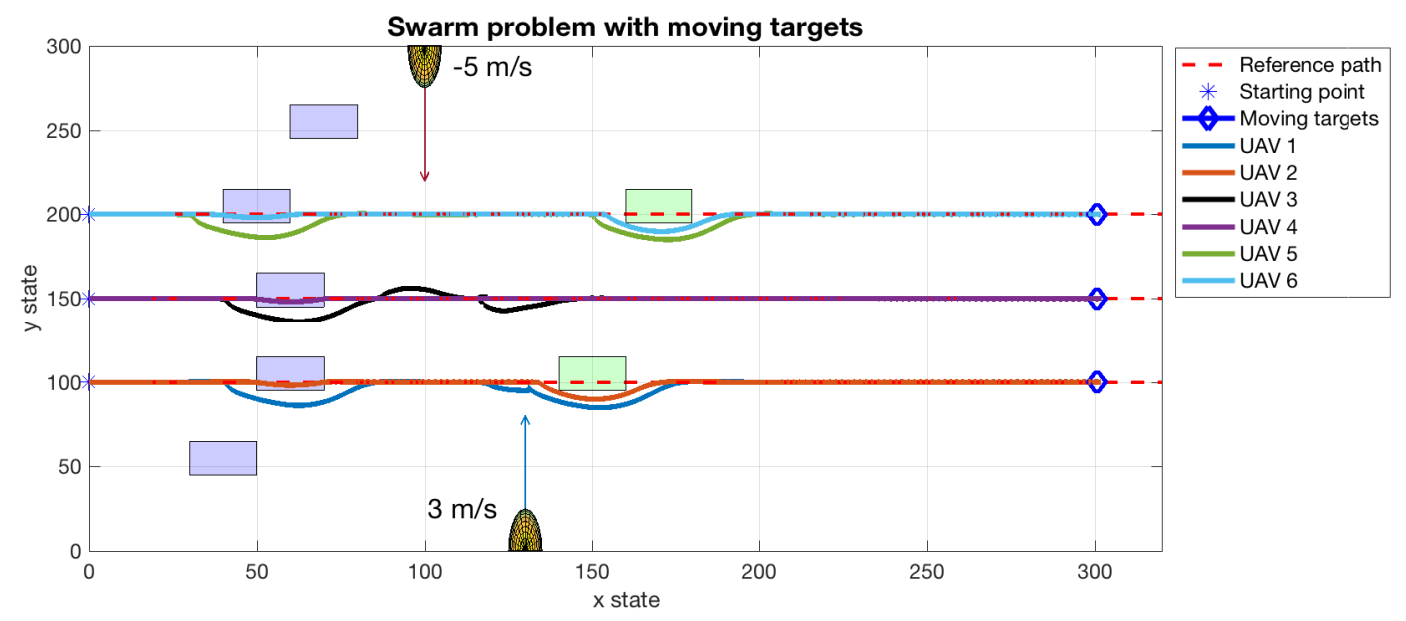

Figure 6.13: XY view of the swarm with moving targets

The Figure 6.14 shows the XZ view of the Figure 6.12, in which it can be seen that the quad-rotors at the height of 40 meters tend to fly over their reference paths at around $x=50$ meters although there is no such obstacle at the height. One of the reasons is due to the robustness function and the penalty parameter that have been chosen (refer to (4.8) and its associated parameter $a_{r e g}=9 / 4$, and $b_{r e g}=4$ from Section 4.4). Besides that, all the UAVs remain close to their reference flight path once obstacles have been avoided. Moreover, from Figures 6.12 to 6.14 it can be seen that, all the UAVs reach very close to the moving targets.

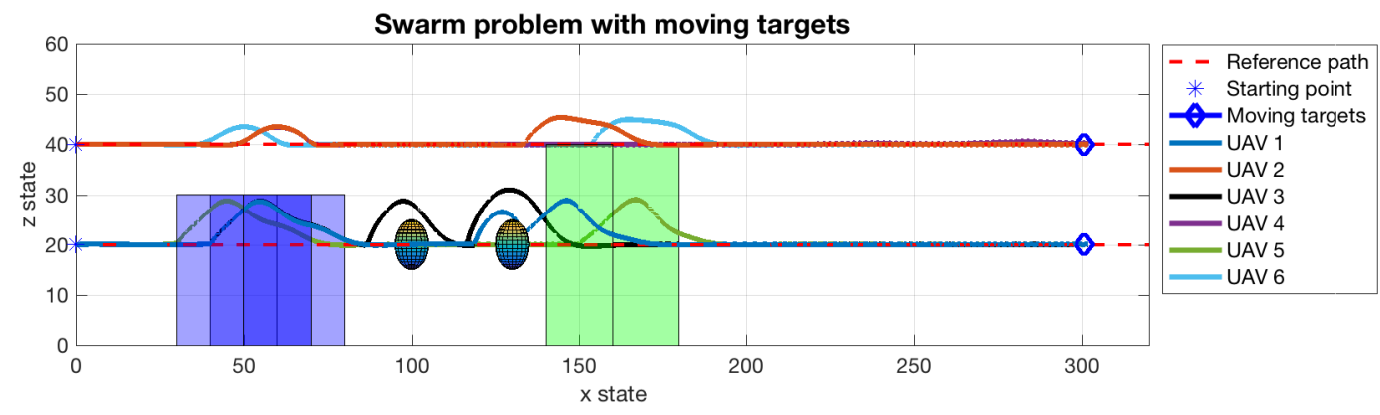

Figure 6.14: XZ view of the swarm with moving targets

Figure 6.15 shows the flight instance at $t=53.6$ seconds, where the UAV 1 and 3 are 
lagging behind compared to other UAVs due to appearance of obstacles, which requires avoidance maneuver. In among all the UAVs, the UAV 4 is very close to its moving target. Also, the UAVs at the top are slightly affected by the robustness function due to stationary obstacles at around $x=50$ meters.

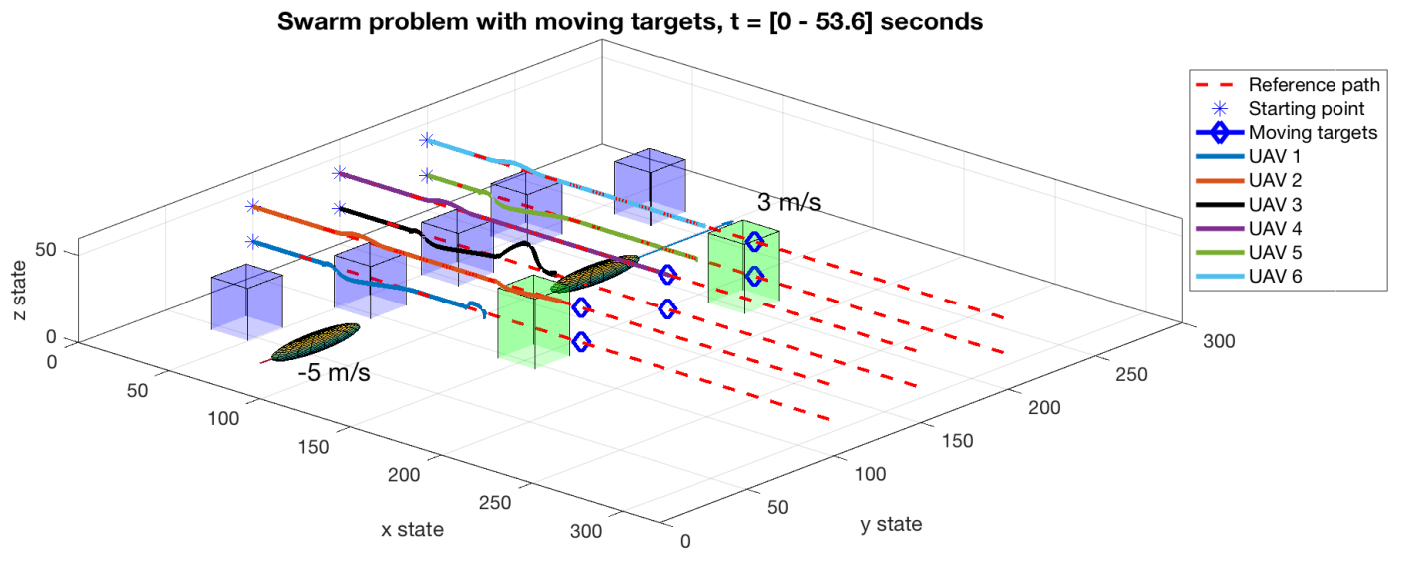

Figure 6.15: The swarm at flight time $t=53.6$ seconds

Similarly, Figure 6.16 shows the trajectories traced by the UAVs from $t=0$ to $t=73.6$ seconds, in which the UAVs at the height of 40 meters reach close to their respective moving targets, whereas, UAV 3 still lags behind.

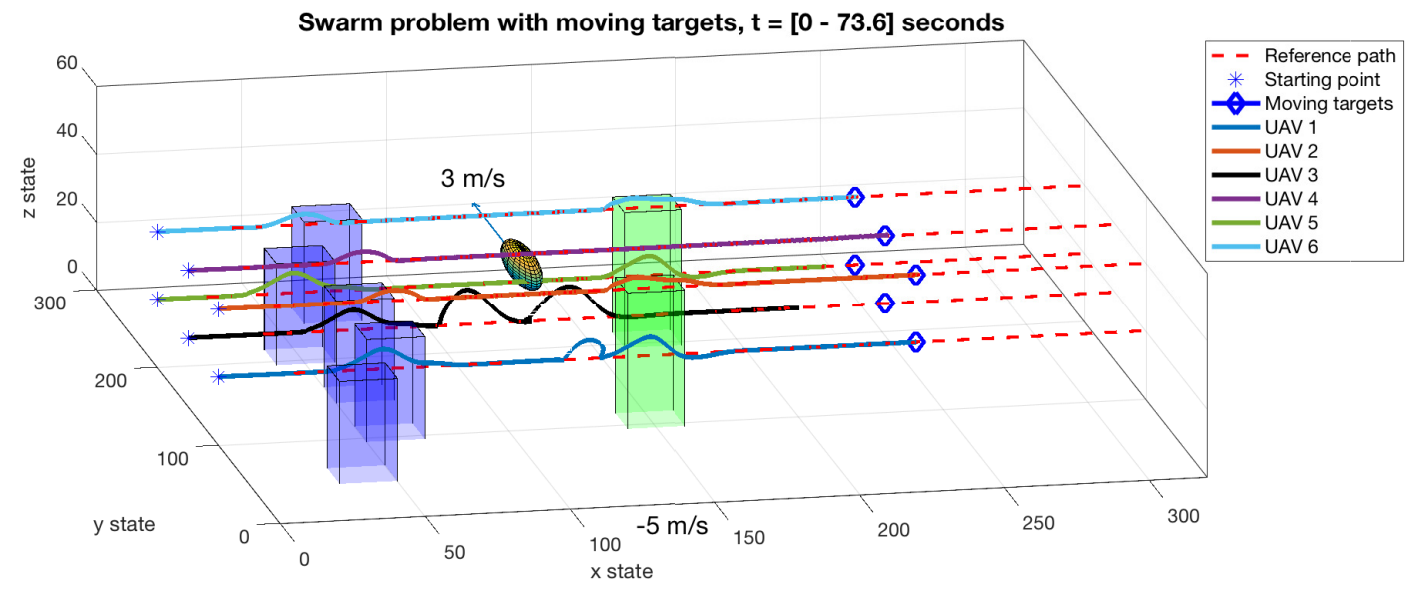

Figure 6.16: The swarm at flight time $t=73.6$ seconds

Finally, Figure 6.17 shows the scenario at the flight time $t=93.6$ seconds, where all the UAVs are very close to their respective moving targets. While, depending on a nature 
of trajectory a UAV takes to avoid obstacles, its flight time is affected, therefore, UAVs facing different obstacles may not progress equally along a desired direction of flight in a swarm. Hence, for a swarm the use of moving targets with their moving velocity slower than UAVs may be helpful in progressing UAVs equally in a desired direction of flight.

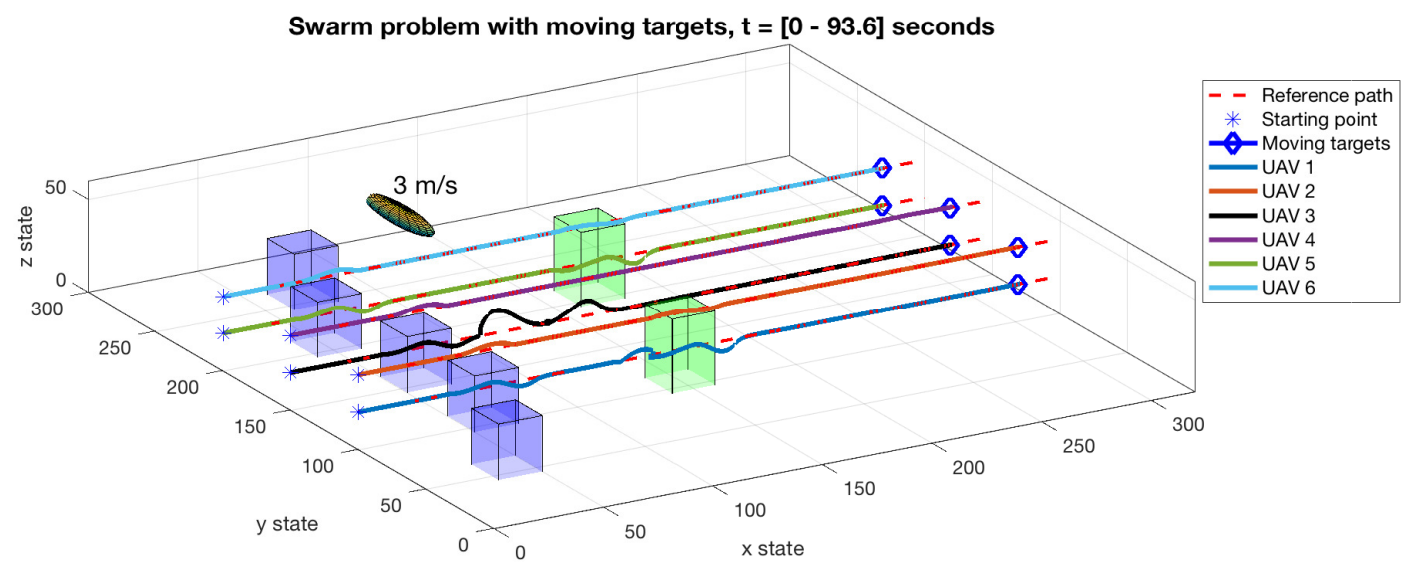

Figure 6.17: The swarm at flight time $t=93.6$ seconds

Figure 6.18 presents the solution time for the swarm example at each model predictive control (MPC) time instant $t$; using a custom solver. It takes 93.6 seconds of flight time for the moving target to reach $x=300$ meters where the simulation is stopped, and by the time all the UAVs almost reach around $x=300$ meters. The maximum solution time for the swarm problem is 295.01 milliseconds while the minimum solution time is 92.61 milliseconds. The solution time for this case of the swarm problem is roughly the same, as that for the case in Section 6.3. This is mainly due to the similar computational complexity between these two cases as the number of nonzeros in their Karush-KuhnTucker (KKT) matrices of their respective quadratic programming (QP)s differ by just $1.57 \%$.

\subsection{Iterative Trajectory Generation for a Swarm with Moving Targets}

Trajectory generation for a swarm with 6 quad-rotors has been shown in Section 6.4 where entire swarm problem is solved at each model predictive control (MPC) time instant. The 


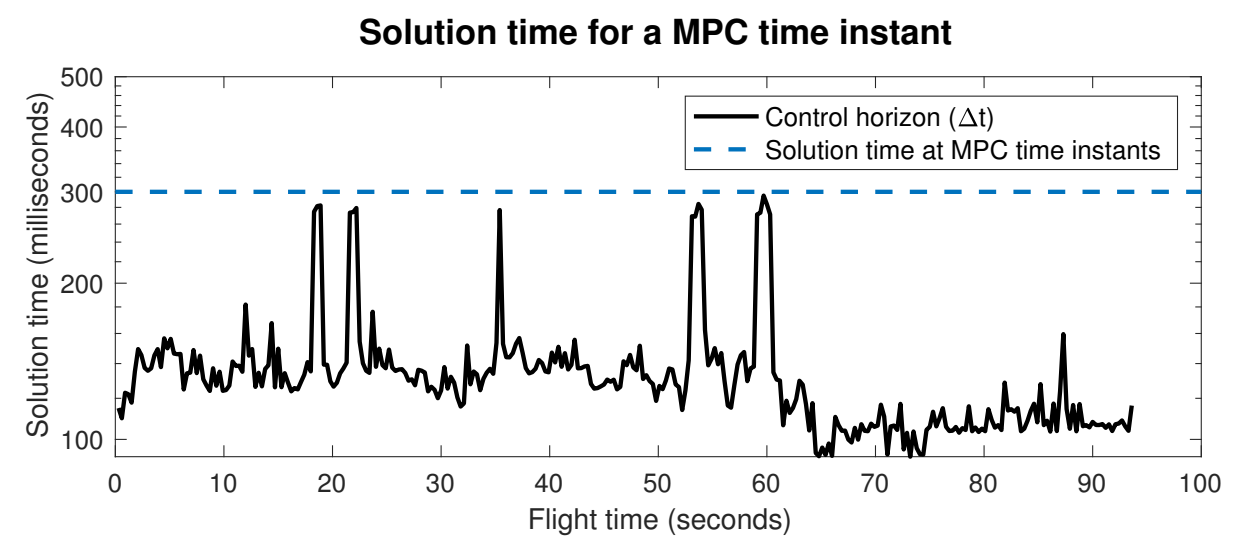

Figure 6.18: Solution time for a MPC time instant for the swarm problem with moving targets

swarm problem SP2-a had all the states, and controls of UAVs as optimization variables in a single problem. The solution time for the case is significantly high, and it may be expected due to the analysis done in Table 6.2. Using the approach, it is expected to reduce the computation time by solving the swarm problem differently. Instead of solving Problem SP2-a directly as in Section 6.4, now an approach to solve the Problem SP2-a is detailed below, where the trajectory generation problem is solved for the swarm, solving iteratively for a UAV in a model predictive control (MPC) time instant considering the trajectories from other UAVs as fixed.

\subsubsection{Problem Definition}

Since most of the required components of the problem definition are already detailed above, a brief definition to the additional component are discussed in this section, which is the solution approach. The solution approach brings a slight changes to the cost function considered. Therefore, the cost for the $j^{\text {th }}$ quad-rotor is defined as,

$$
J\left(X^{j}(\cdot), U^{j}(\cdot)\right)=\int_{\tau_{0}}^{\tau_{f}} F\left(X^{j}(t), U^{j}(t), t\right) d t
$$

where the expression and definition of $F\left(X^{j}(t), U^{j}(t), t\right)$ are from (6.7).

The problem for this case is defined as, 


\section{Problem APj:}

Given an initial condition $\left(x_{0}^{j}, y_{0}^{j}, z_{0}^{j}\right)$ for a $j^{\text {th }}$ quad-rotor at time $\tau_{0}$, find the control-state pair $(X(t), U(t))$ for the swarm, to minimize the cost (6.8) for a $j^{\text {th }}$ quad-rotor while trajectories of other UAVs in the swarm are fixed, subject to

$$
\begin{array}{ll}
\text { UAV model (5.4) as, } & \dot{X}^{j}=f\left(X^{j}, U^{j}\right) \\
\text { Obstacle constraint in (6.2) as, } & h_{i}^{j}(x, y, z) \geq \epsilon, \forall i=1, \ldots, O \\
\text { Collision avoidance constraint (6.1) } & \text { for } j^{\text {th } \mathrm{UAV}} \\
\text { Thrust constraint (T as (5.14)) } & T^{j} \leq T_{\max } \\
X^{j} \in X & \\
U^{j} \in U &
\end{array}
$$

Once APj is defined, the swarm problem is solved as follows,

\section{Problem SP2-b:}

$$
\text { for } \begin{aligned}
j & =1: N_{s} \\
& \text { Solve } A P j \\
& \text { Update } X, U
\end{aligned}
$$

end

Using a model predictive control (MPC) framework the swarm problem SP2-b is solved, in which $X^{j}$ and $U^{j}$ are design variables for iteration $j$, while the trajectories for the other UAVs are considered fixed. At each model predictive control (MPC) window, $\mathrm{APj}$ is solved iteratively for each UAV where the problem is initialized at the solution of previous model predictive control (MPC) window as outlined in Section 4.3. The sequential convex programming ( $\mathrm{SCP}$ ) approach from Chapter 2 is used to solve $\mathrm{APj}$ where the maximum number of sequential convex programming (SCP) iteration have been set to 5 .

\subsubsection{Numerical Example}

The same example scenario is considered with exactly the same problem parameters as used in Section 6.4.2. Figure 6.19 shows the resulting trajectories for the swarm scenario when trajectories where computed iteratively for UAVs at each model predictive control 
(MPC) time instant in the swarm. In the figure, two moving obstacles are shown in their initial position.

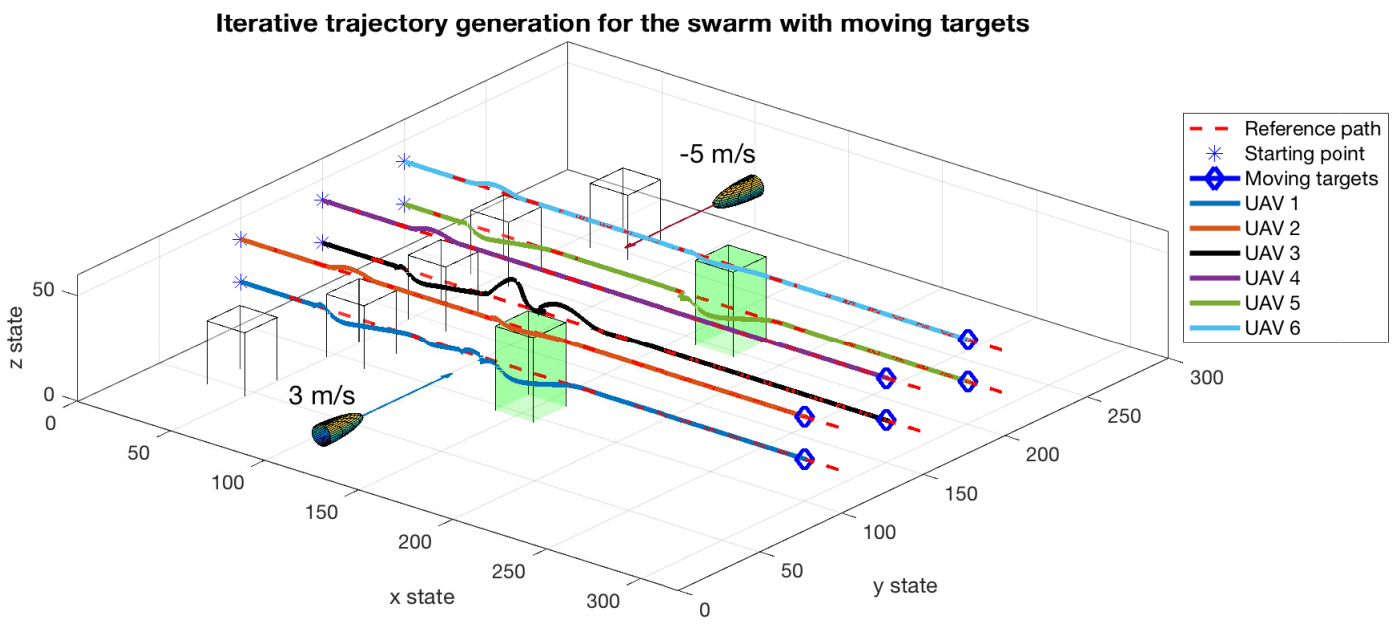

Figure 6.19: 3D view for the swarm with moving targets

While both of the problems SP2-a and SP2-b solve the same swarm scenario, their resulting trajectories differ. Although, the flight time and behaviour of UAVs in terms of keeping with moving targets remains almost the same, their trajectories do not match exactly the same. In Figures 6.20 and 6.21, the trajectories Magenta in colour is the result of solving Problem SP2-a while the black in colour is from Problem SP2-b.

While some UAVs may have almost the same trajectories between the problems, some do not, as it can be seen in Figure 6.21, and this align with the propositions from [121] where in the case of obstacles the trajectories between the problems may not be the same.

Figure 6.22 shows the distance maintained between UAVs at the height of 20 and 40 meters respectively for the numerical example of SP2-b. Since, UAVs along $y$ - axis are 50 meters apart along with their reference path, they do not come as close as 20 meters. However, UAVs along $z$ - axis are initiated 20 meters apart, therefore, a maneuver by a UAV should impact other UAV along $z$-axis because of the minimum distance they have to maintain which is 19 meters. In the figure, the distance between UAV:1 $\sim 2$, $\mathrm{UAV}: 3 \sim 4$, and UAV:5 $\sim 6$ throughout the fight are plotted. Although, from Figure 6.21 we may feel that the UAVs along the $z$-axis might be closer than 19 meters in between $x=0 \sim 200$ meters, it is definitely not the case which is clear from Figure 6.22. In 


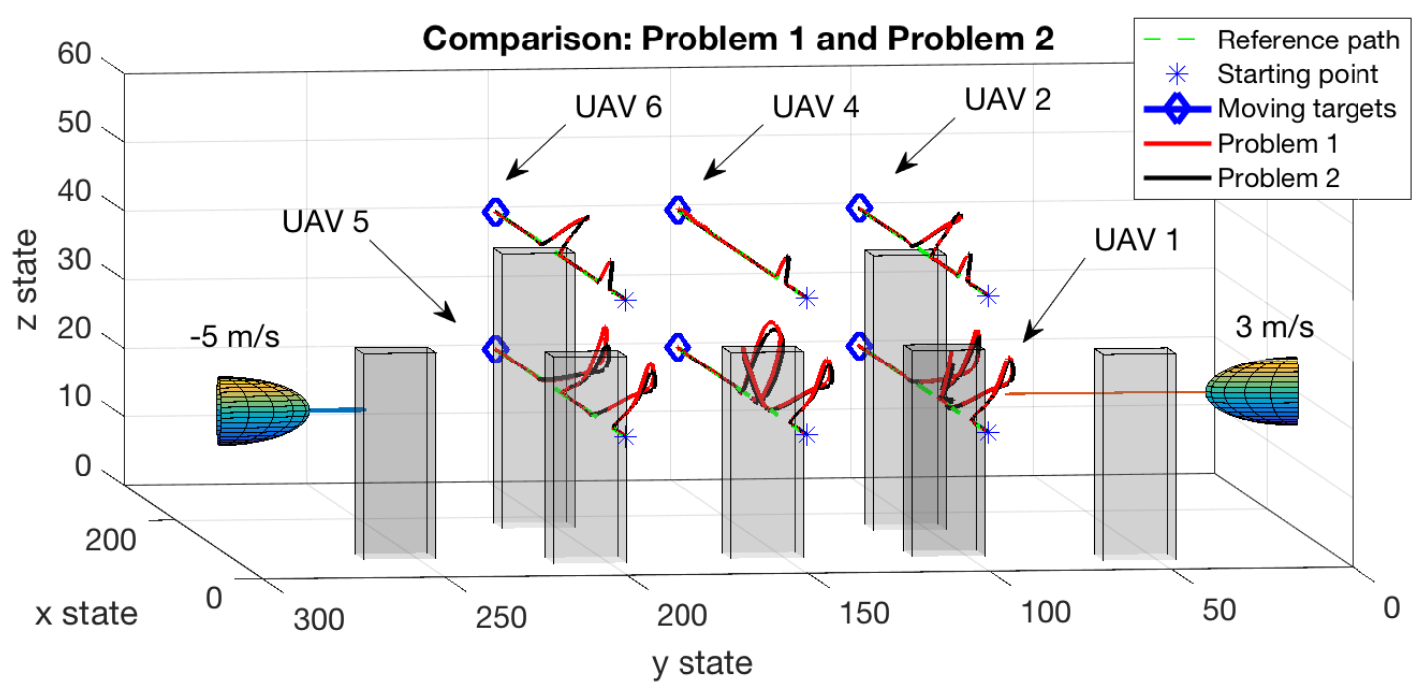

Figure 6.20: 3D view for the swarm with moving targets

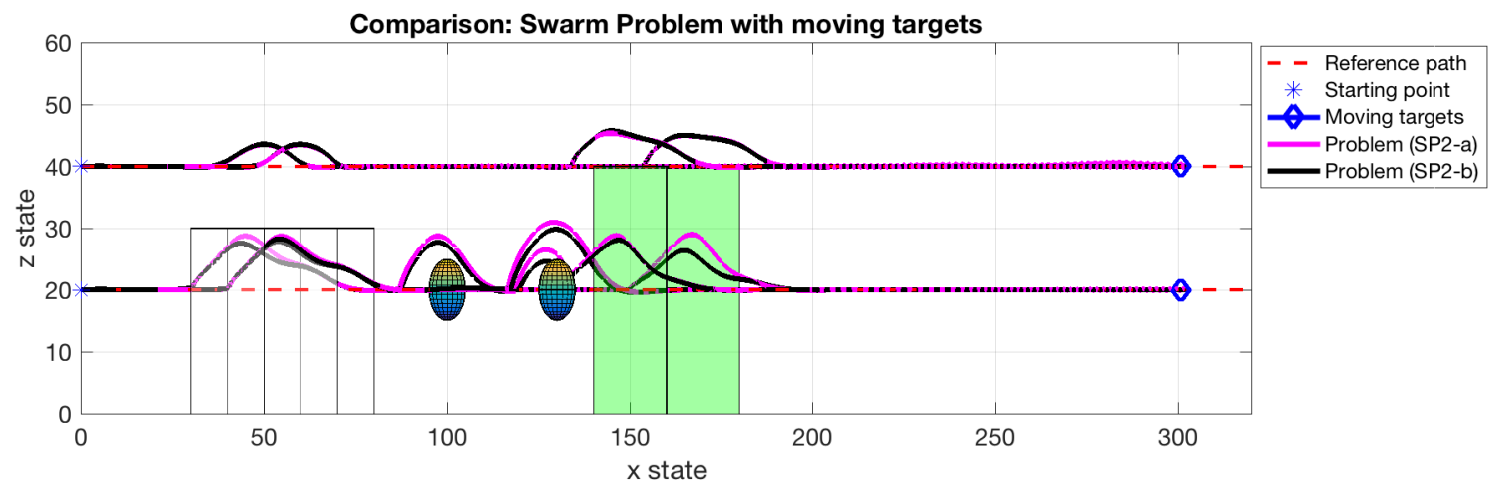

Figure 6.21: XZ view for the swarm with moving targets

contrast to the plots from Problem SP1 where UAVs progress equally along the direction of flight (because UAVs in the case are far apart and even with maneuver required they do not come as close as 20 meters), in this current case the UAVs do not progress equally along the desired direction of flight, specially in the presence of obstacles which is clear from figures 6.16 and 6.15 , and one of the reasons is to maintain the minimum distance of 19 meters between UAVs along $z$-axis.

Figure 6.23 compares the solution time for problems SP2-a, APj and SP2-b for their respective example simulation. In the figure, it could be seen that the solution time for a UAV at model predictive control (MPC) time instant for Problem APj is approximately 


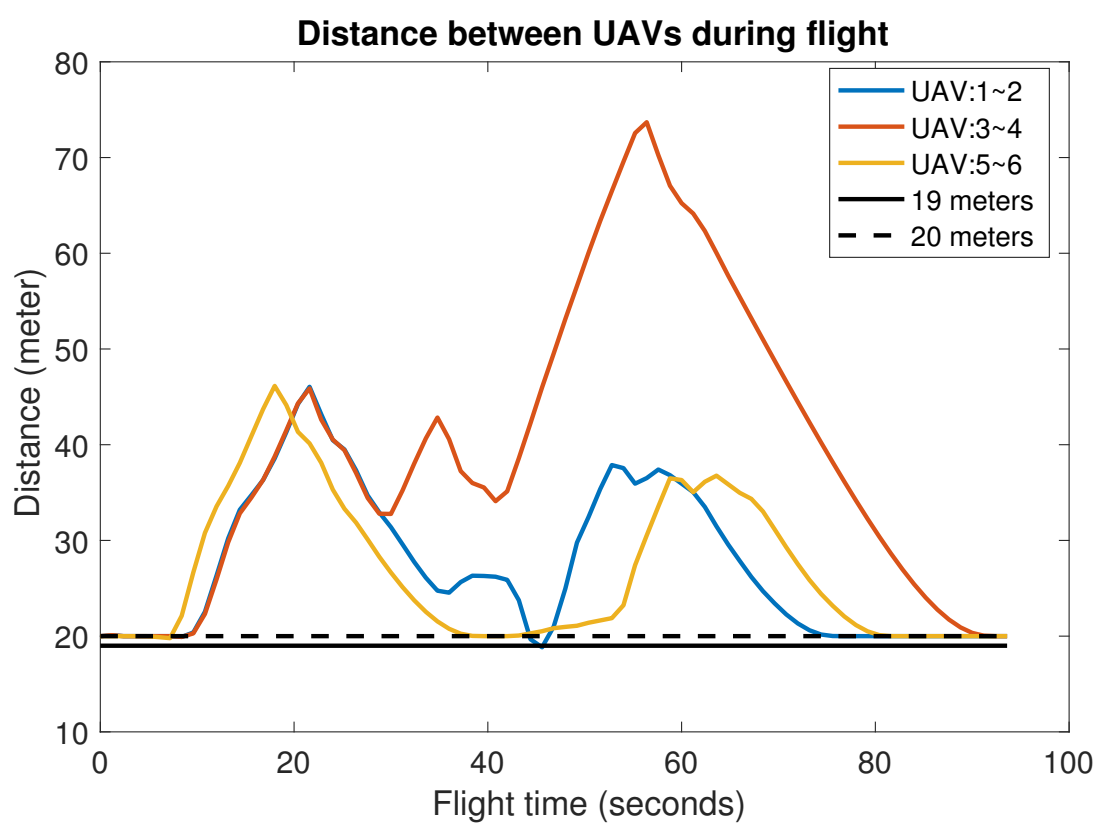

Figure 6.22: Distance bewteen UAVs

4 milliseconds in average with maximum of 10.5 milliseconds. Thus the total solution time for all the UAVs sums to 26.40 milliseconds at the maximum at a model predictive control (MPC) time instant for Problem SP2-b, and 15.93 milliseconds at the minimum. This is due to the reduction in the computational complexity with total nonzero of 6189 in the Karush-Kuhn-Tucker (KKT) matrix of its quadratic programming (QP) compared to the case of SP2-a where the solution time is more than 92,61 milliseconds for all the instances, with the maximum of 295.01 milliseconds.

From the comparison in the solution time in Figure 6.23, it can be seen that the solution time for the swarm problem that iteratively solves for a UAV at a time in a model predictive control (MPC) time instant is much faster compared to the problem that solves for the entire UAVs at once. While both problems SP2-a and SP2-b solve the same swarm scenario they do not obtain the same solution, however the formulation for Problem SP2-b has an advantage in terms of solution time. In terms of average solution time, Problem SP2-b with the average solution time of 19.6 milliseconds is more than seven times faster than the Problem SP2-a with the average solution time of 144.3 milliseconds. 


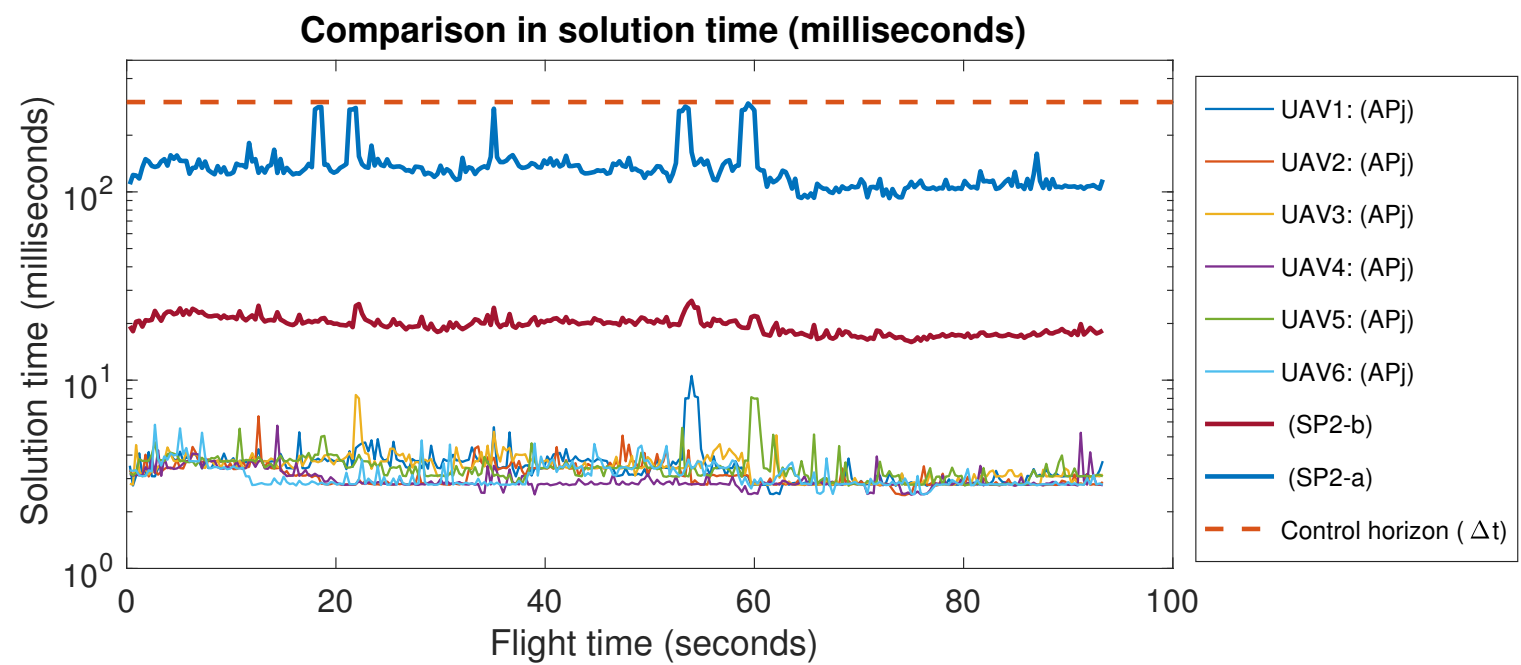

Figure 6.23: Solution time comparison between swarm problems with moving targets

\section{Comparison with ECOS and IPOPT}

As similar to the Section 5.3.2, the trajectory generation (from Section 6.5.2) for the swarm is solved with ECOS and IPOPT, and then compared in terms of the value of cost and the computation time. Since, the trajectory generation is for the swarm, the value of cost for all the UAVs are added together to signify as the total cost. In the comparison, CS and IPOPT stands very close in terms of the value of the cost, while the ECOS produces trajectory with more deviation. In terms of the computation time, CS is at least five times faster than ECOS and IPOPT. While the ECOS is faster than IPOPT, its value for the cost is nearly four times higher than that of IPOPT.

Table 6.6: Comparison of custom solver (CS) with IPOPT and ECOS.

\begin{tabular}{cccc}
\hline \hline & Cost & Average computation time $(\mathrm{ms})$ & Maximum computation time (ms) \\
\hline IPOPT & 36.2 & 1076.7 & 1183.7 \\
\hline ECOS & 144.4 & 111.3 & 186.3 \\
\hline CS & 36.0 & 19.6 & 26.4 \\
\hline \hline
\end{tabular}




\subsubsection{Monte-Carlo Test}

The above swarm example with moving targets may not be a sufficient demonstration of real-time computability of the swarm problem. Therefore, in this section a MonteCarlo simulation is performed, with uniformly distributed random obstacles but with the same problem definition as in this Section, that includes quad-rotor parameters as in Appendix 4.1, bounds on optimization variables as in Appendix 4.3, and the additional parameters from Table 5.1 where necessary. As similar to the Monte-Carlo example in Section 5.3.3, 100 random test are performed where the locations of the obstacles and their parameters (that defines its location, shape, and size) are randomly initialized with uniform distribution as in Section 5.3.3. Likewise, the simulation is terminated once moving targets reach $x=800$ meters. In the following the results are summarized.

In Figure 6.24 it can be observed that the solution time for a quad-rotor is much lower than the sampling time. Although the solution time for a swarm is much more important than for a UAV, but from the approach that has been undertaken, the solution time for a swarm is approximately at the multiples of UAVs, as the problem size that are solved iteratively for a UAV is the same for all UAVs in the swarm. Figure 6.24 shows that for a little over $20 \%$ of the instances in the Monte-Carlo test, custom solver takes 2-4 milliseconds of solution time for a quad-rotor, while for approximately $76 \%$ of the instances the solver takes from $4 \sim 6$ milliseconds of solution time. The number of nonzero entries in the Karush-Kuhn-Tucker (KKT) matrix of a quadratic programming (QP) problem posed in this test is 8279, as such the computational complexity of this problem seems roughly as similar to $\mathrm{APj}$ with the variation of $1 \sim 2$ milliseconds solution time.

Finally, to understand the solution time offered by the custom solver for the swarm in the Monte-Carlo, the results are plotted in Figure 6.25. The figure shows that, all the instances in the test are solvable within 30 milliseconds using the custom solver in the laptop computer. As similar to the result from Figure 6.23, the solution time remains almost consistent using the approach for the same number of UAVs.

\subsubsection{Scalability of the Swarm Problem}

Encouraged by the solution time of the approach in SP2-b, its scalability is studied with different number of UAVs in the swarm. For this study the same obstacle configuration 


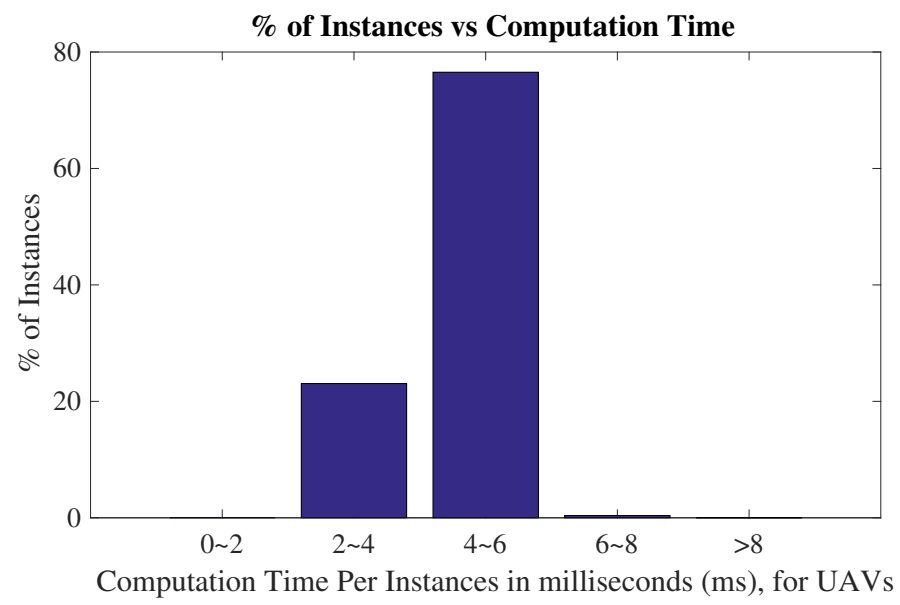

Figure 6.24: Computation time per instances a UAV

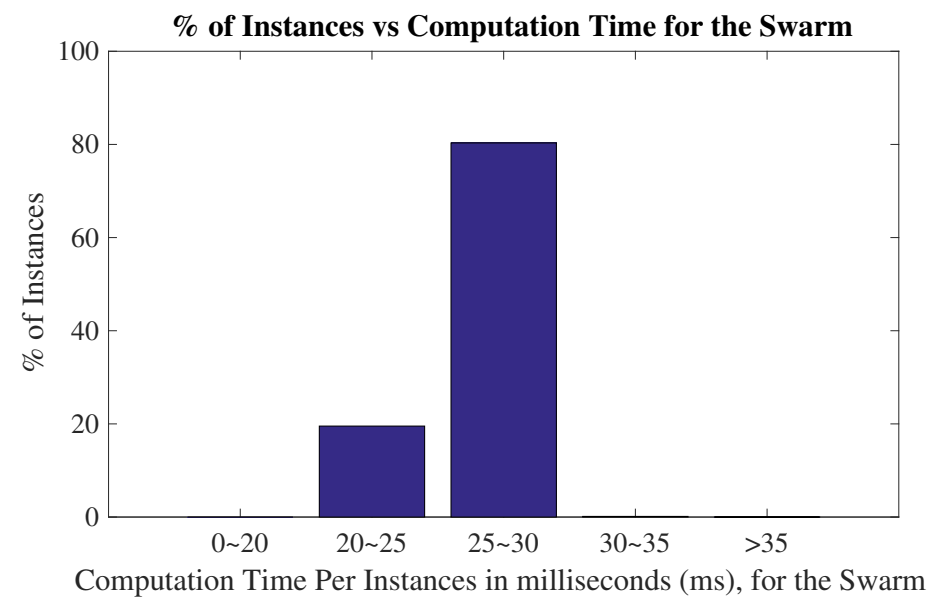

Figure 6.25: Computation time per instances for UAVs

as in Section 6.4.2 is used, whereas the starting point for UAVs are distributed along $y$-axis and $z$-axis at $x=0$ meters with equal distances between them. Moreover, a reference path for each UAV is defined such that it extends parallel from the starting point of each UAV along the $x$-axis. In addition to the swarm study with 6 quadrotors already done, the swarm problems with 16,32 and 64 quad-rotors are solved and compared in terms of the solution time. For the case of $16 \mathrm{UAVs}$, the swarm is initialized such that they are 30 meters apart along $y$ and $z$-axes with the first one at $(0,30,30)$ meters, while for the swarm problem with 32 and 64 quad-rotors, the are initialized 20 
meters apart along $y$ and $z$-axes with the first one at $(0,20,20)$ meters. As similar to the example in Section 6.4.2, a simulation is terminated once moving targets reach $x=300$ meters.

Figure 6.26 shows the solution time for the swarm problems. While the solution time for the swarm increases with the increasing number of UAVs, it is notable from Table 6.7 that the average solution time for APj does not scale linearly with the increasing number of UAVs in the swarm. From the study the following results are noted, the maximum solution time for a model predictive control (MPC) time instance to solve the swarm problems with 6, 16, 32 and $64 \mathrm{UAVs}$ are 26.40, 73.16, 194.24, and 556.13 milliseconds respectively. It is also notable that the laptop computer is at least able to generate realtime trajectories for up to 32 quad-rotor UAVs within the allocated sampling time which is set to 300 milliseconds.

\section{Comparison in solution time (milliseconds)}

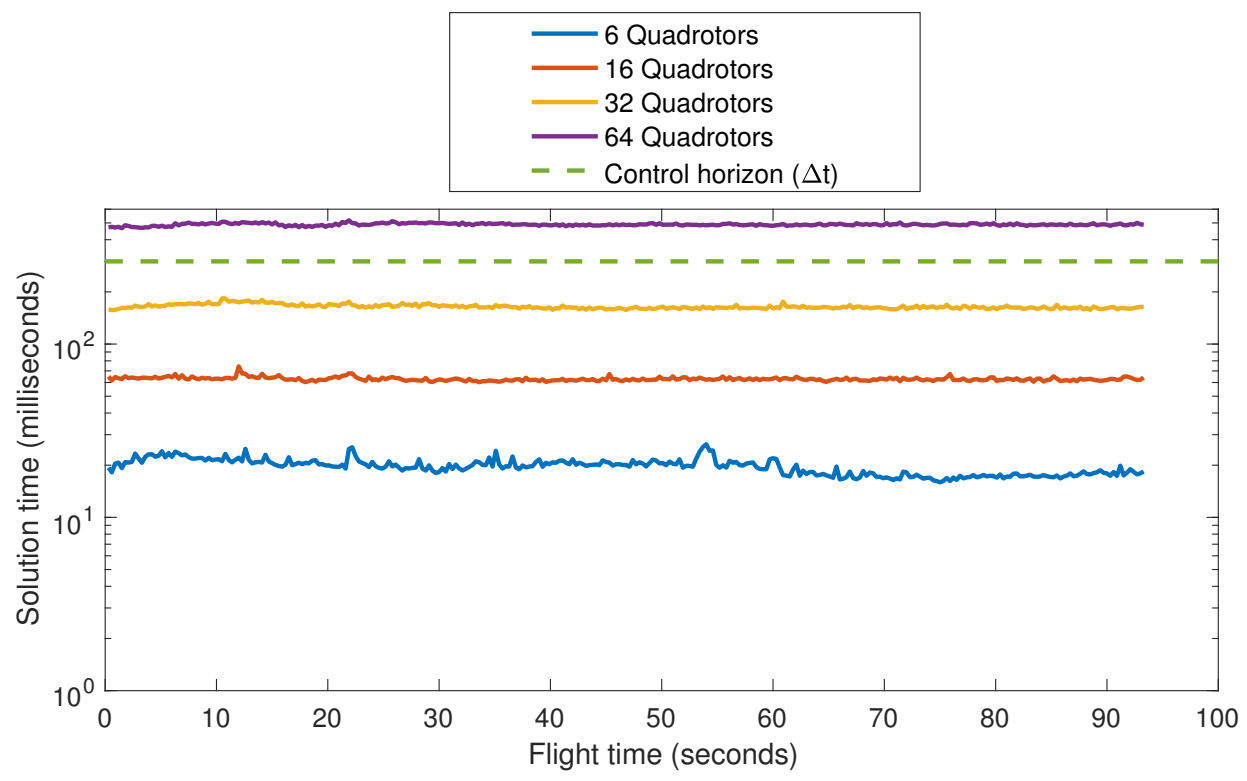

Figure 6.26: Computation time for various swarm size

Table 6.7 presents the average solution times along with problem complexity for comparison. In the table, $A v g_{t q}$ represents the average solution time for $\mathrm{APj}, A v g_{t s w}$ refers to the average solution time for Problem SP2-b, and $N n z_{\text {problem }}$ is the number of non-zero in the Karush-Kuhn-Tucker (KKT) system of a quadratic programming (QP) sub-problem with the $\mathrm{APj}$, this also represents the problems computational complexity 
as the solution time substantially depends on the amount of non-zero in the KarushKuhn-Tucker (KKT) system. In the table, it is observable that the $A v g_{t q}$ does not grow linearly with UAVs, as such it is directly related to $N n z_{\text {problem }}$, this means that the problem size does not double up at doubling the number of quad-rotors in the swarm. However, $A v g_{t s w}$ grows little over the linear rate with the number of UAVs.

Table 6.7: Swarm problems with their average solution time

\begin{tabular}{cccc}
\hline \hline Quad-rotors & $A v g_{t q}$ & $A v g_{\text {tsw }}$ & $N n z_{\text {problem }}$ \\
\hline 6 & 3.49 & 20.94 & 6189 \\
16 & 3.94 & 63.01 & 7937 \\
32 & 5.17 & 165.48 & 10977 \\
64 & 7.65 & 489.75 & 17057 \\
\hline \hline
\end{tabular}

\subsection{Summary}

The chapter presented a study on trajectory generation for a swarm of quad-rotor UAVs with two different attributes. The first, trajectory generation for a swarm of quadrotor without a goal point. The next, trajectory generation for a swarm of quad-rotors with moving targets. The problems defined can represent scenarios for various obstaclerich environments. In order to reduce the computation time for trajectory generation problems for a swarm, a custom solver is used. In this chapter, the solution time between the swarm problems with moving targets are compared, in which a problem is solved iteratively for a UAV at a time while keeping the trajectories from other UAVs fixed, versus the same problem where in a model predictive control (MPC) time window an entire swarm problem is at once. Also, it was seen that the swarm problem that solves iteratively for a UAV at a time in a model predictive control (MPC) window is much faster than the problem that solves for all UAVs at once at that window. From the Monte-Carlo test for the swarm with $6 \mathrm{UAVs}$, the consistency in the solution time was noted, where the swarm problem with the approach is solvable within 30 milliseconds. Results from the scalability study show that the swarm problem with 32 quad-rotors are solvable within the sampling time in the laptop computer. 


\section{Chapter 7}

\section{Conclusion and Future Works}

This dissertation presented methods for real-time trajectory generation and autonomous obstacle avoidance for UAVs and the extension of approaches for swarm application of quad-rotors. The dissertation concludes the following.

\subsection{Conclusion}

The first and the second methods for customization of solver offer an efficient approach to solve a KKT system of a small and a large size quadratic programming (QP) problem with the positive semi-definite Hessian respectively. These approaches outlined in Chapter 2 offer a rapid solution to quadratic programming (QP) problems in Chapters 3 to 6 .

An approach for rapid feasible trajectory generation for a fixed-wing UAV is presented in Chapter 3 whereby the dynamic equation of a fixed-wing UAV is transformed to a simplified equation from which the bounds on the simplified controls are derived. The use of a custom solver in conjunction with the method reduced the solution time to tens of milliseconds for a laptop computer, making the approach applicable for rapid feasible trajectory generation. Moreover, the method can be used to find a feasible starting point for solving the original problem, thus reducing the total computation time.

The rapid generation of trajectory and autonomous obstacle avoidance for a fixedwing UAV is shown in Chapter 4, where a comparative study between the kinematic and the dynamic model for the rapid generation of avoidance trajectory is presented. From the comparisons shown, the dynamic model performs better in terms of maneuverability, 
computation time and the total flight time compared to the kinematic model, where a custom solver solves the problems. The trajectory generated using the dynamic model deviates less from the desired reference path compared to the kinematic model even in the presence of the wind disturbance, making the trajectory generation more robust compared to with kinematic counterpart. Also, the solution time in a computing platform like Raspberry PI-3B implies that the method is applicable for rapid avoidance trajectory generation in a credit-card sized computer.

In Chapter 5, real-time trajectory generation and autonomous obstacle avoidance using the finite horizon model predictive control for the case of quad-rotor UAVs was examined. A comparable study between the use of the kinematic and the dynamic model for a quad-rotor UAV shows that the dynamic model performs slightly better than the kinematic model while both of them have approximately the same value of deviation for 95\% of instances in Monte-Carlo test, whereas the kinematic model takes almost half the computation time than the dynamic model. Therefore, in contrast to the case of a fixedwing UAV, the quad-rotor UAV with the kinematic model performs reasonably better in comparison to with the quad-rotor dynamic model with the benefits of the solution time, using custom solver.

An application of a swarm of quad-rotors using the finite horizon model predictive control and a custom solver was studied in Chapter 6. The swarm problem with moving targets was studied in two sub-problems; the first approach, solve the entire swarm problem at once in a model predictive control (MPC) window, and the second approach, solve iteratively for UAVs at a time within a model predictive control (MPC) window. The comparative study between the sub-problems indicate, solving by the second approach is almost four to seven times computationally cheaper than solving for the entire swarm at once. From the Monte-Carlo test for the swarm with 6 UAVs, it is easier to node the consistency in the solution time where the swarm problem is solvable within 30 milliseconds (using the second approach). Results from the scalability study show that the swarm problem with 32 quad-rotors are solvable within the sampling time in the laptop computer. 


\subsection{Future Works}

The methods developed for the application in this dissertation are ready for implementation as long as the computer onboard the UAV is as computationally capable as the Raspberry PI 3B computer or better. However, there may arise unforeseen challenges with implementation on a complete UAV system. Since implementation in a UAV system would take a generated trajectory from the methods in this dissertation and feed that into an autopilot, the autopilot would be responsible for trajectory tracking. Therefore, an obviously expected work is to determine the UAVs flight constraints in which it will be implemented. Regarding an implementation of the method for trajectory generation for a swarm quad-rotors, the communication between a central computer and quad-rotors may be crucial. The following are potential future works for the integration of the trajectory generation methods developed in this dissertation to a complete UAV system.

- Hardware implementation of rapid feasible trajectory generation for fixed-wing UAVs in obstacle environment.

- Embed the finite horizon model predictive control method for trajectory generation along with a custom solver presented in this dissertation for experimental validation of the problem formulations.

- Experimental validation of the methods for trajectory generation for a swarm of quad-rotors.

- Study high fidelity model for UAVs in order to generate aggressive trajectory for real-time application using the custom solver (within model predictive control framework.) 
Appendices 


\section{Appendix 1}

\section{Customization of Solver, Examples}

\subsection{Custom Factorization Example: First Approach}

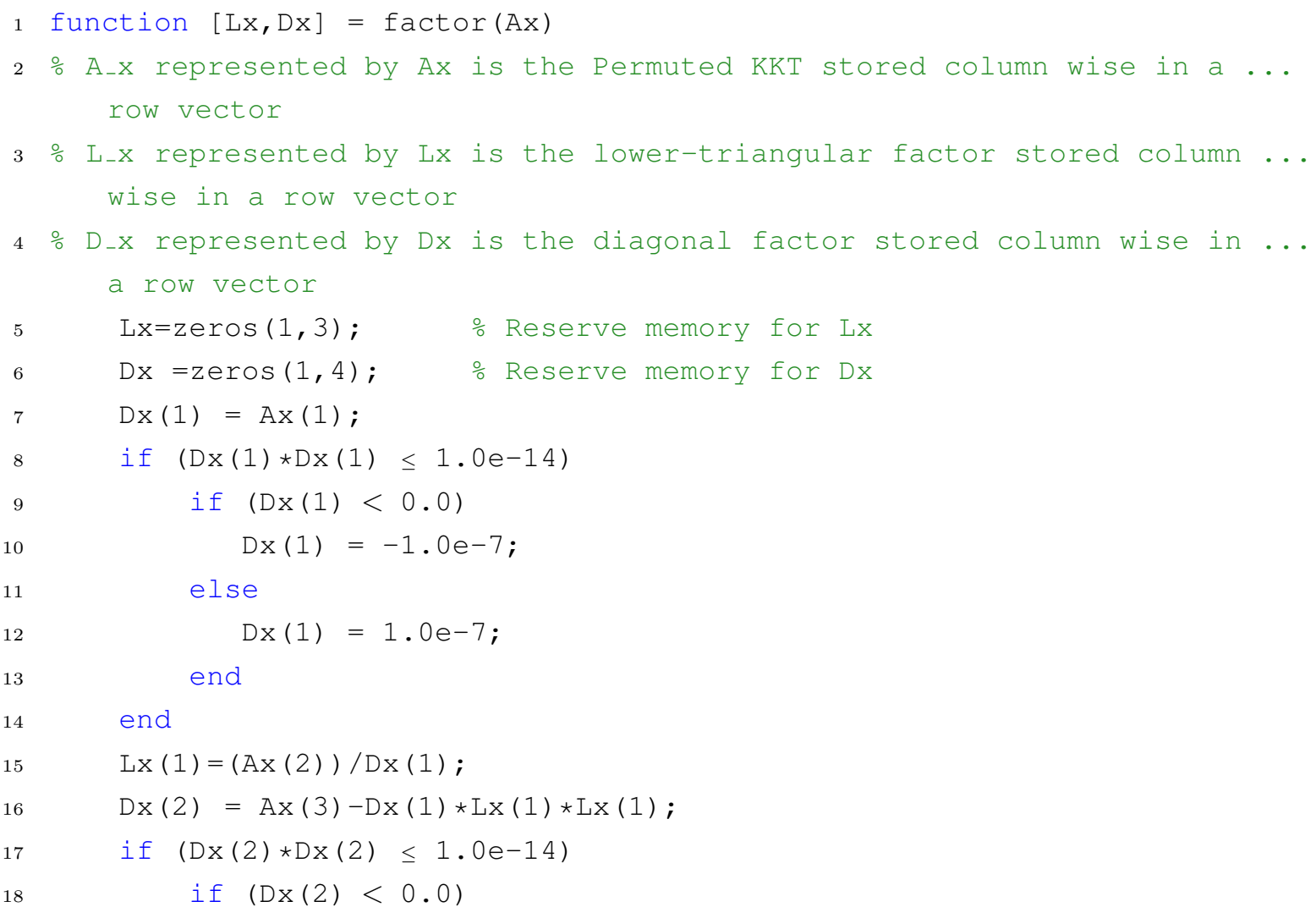




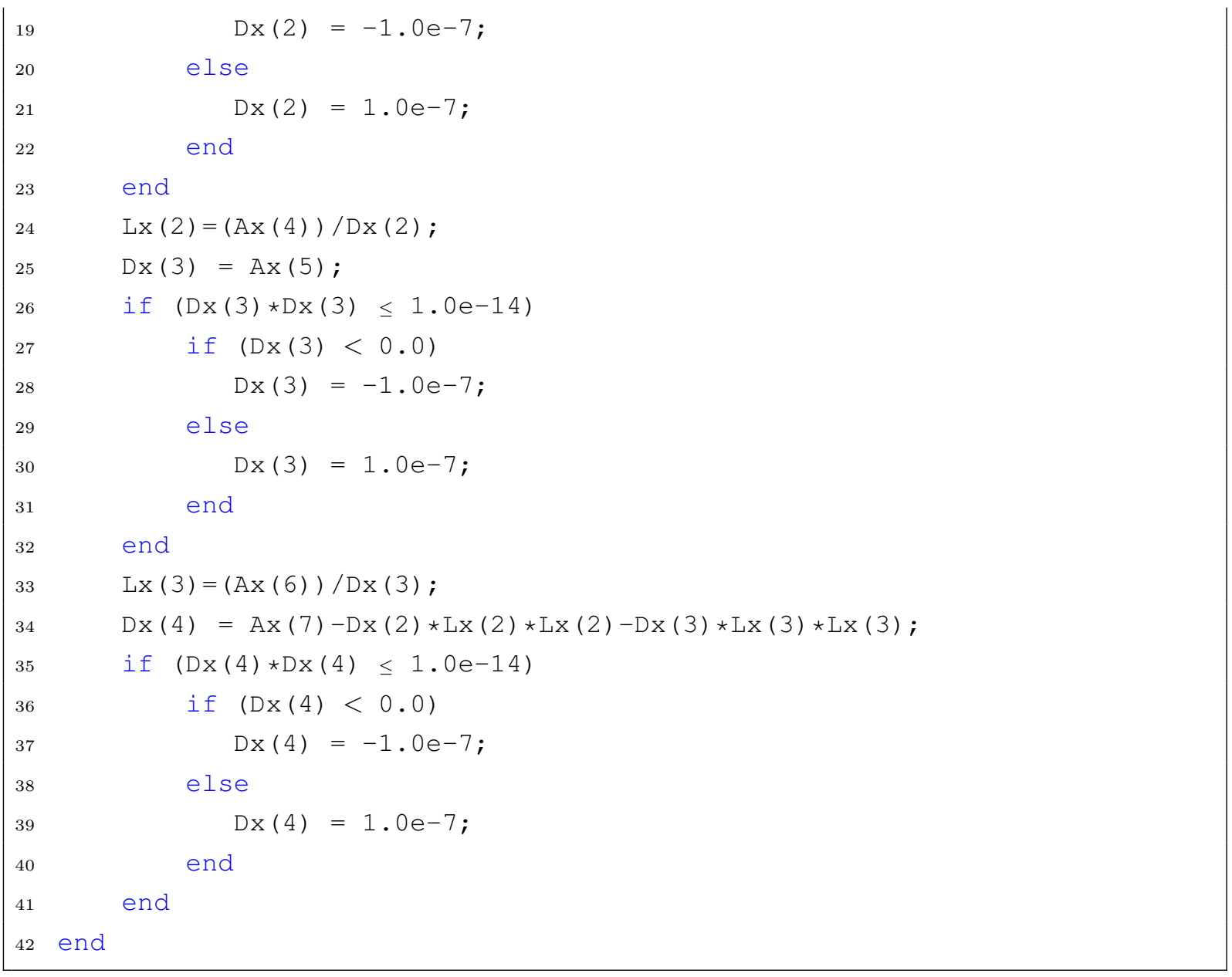

\subsection{Custom Solve Example: First Approach}

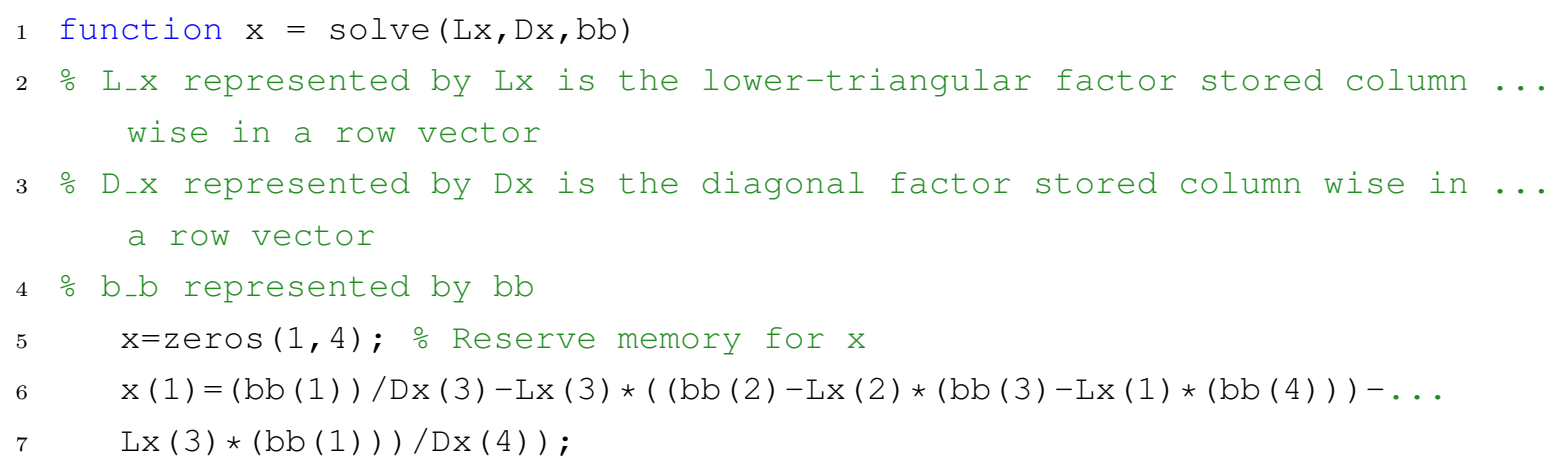




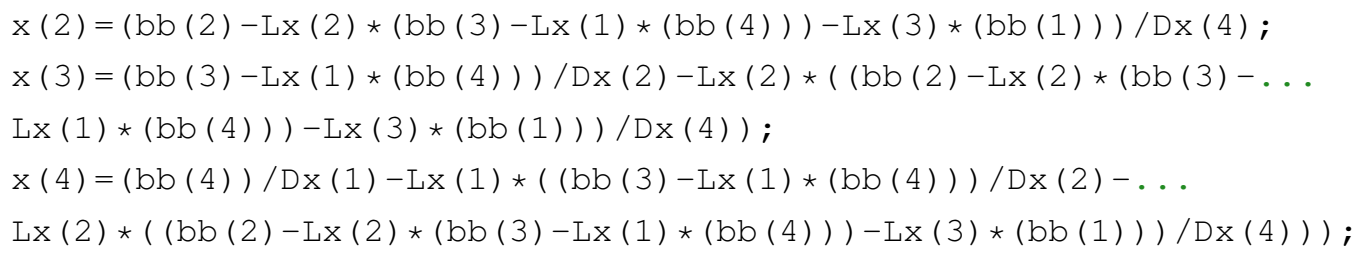

\subsection{Custom Factorization Example: Second Approach}

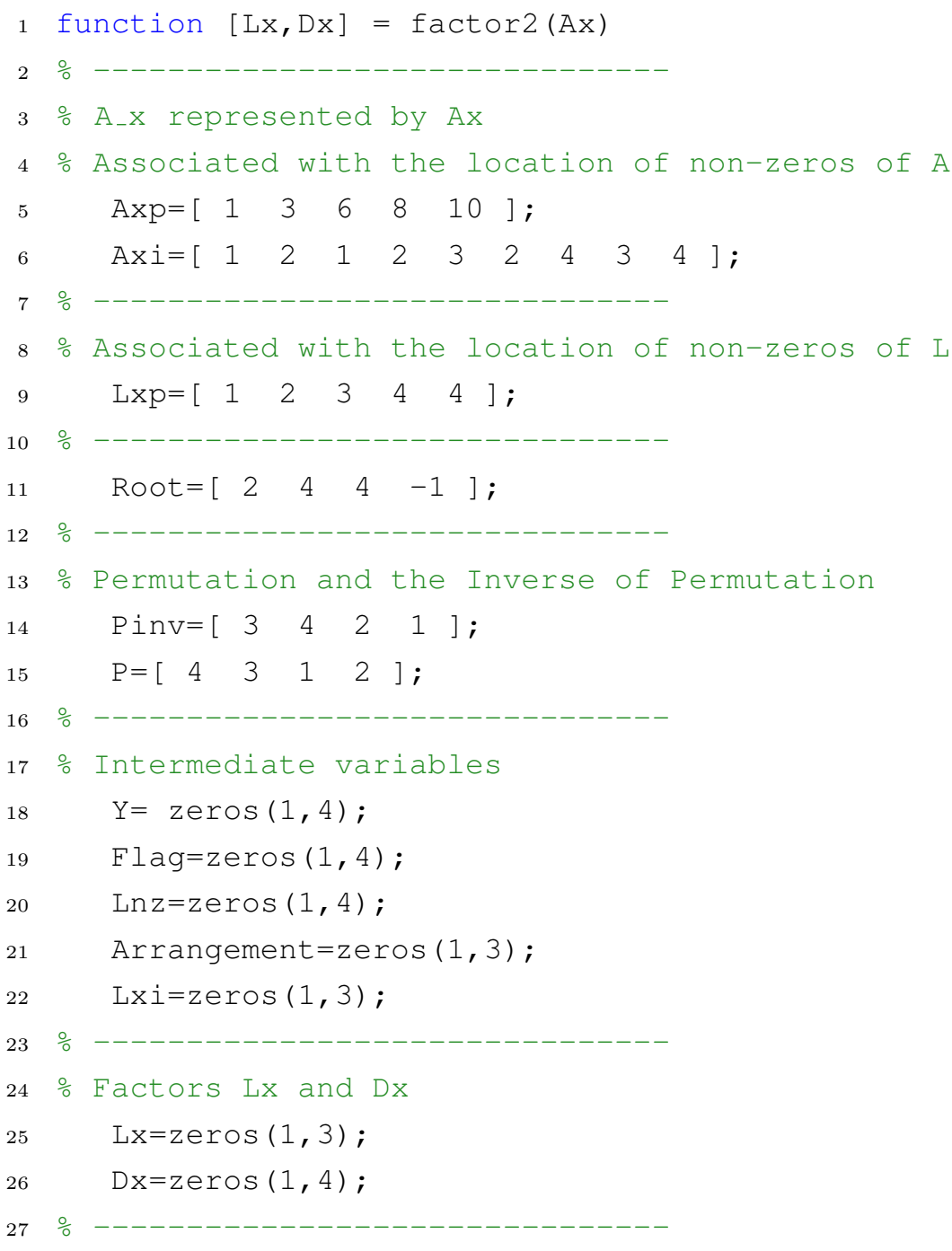




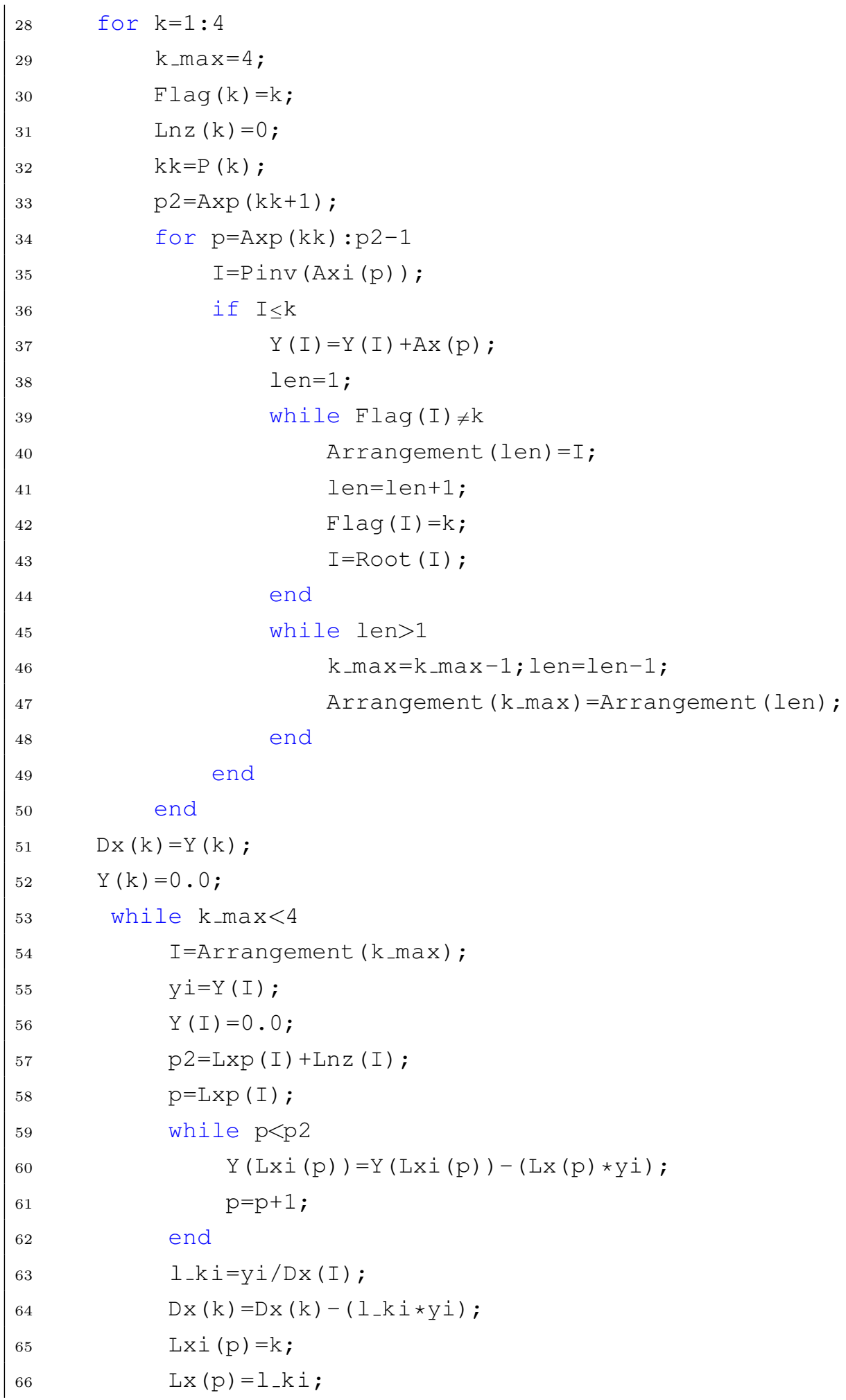




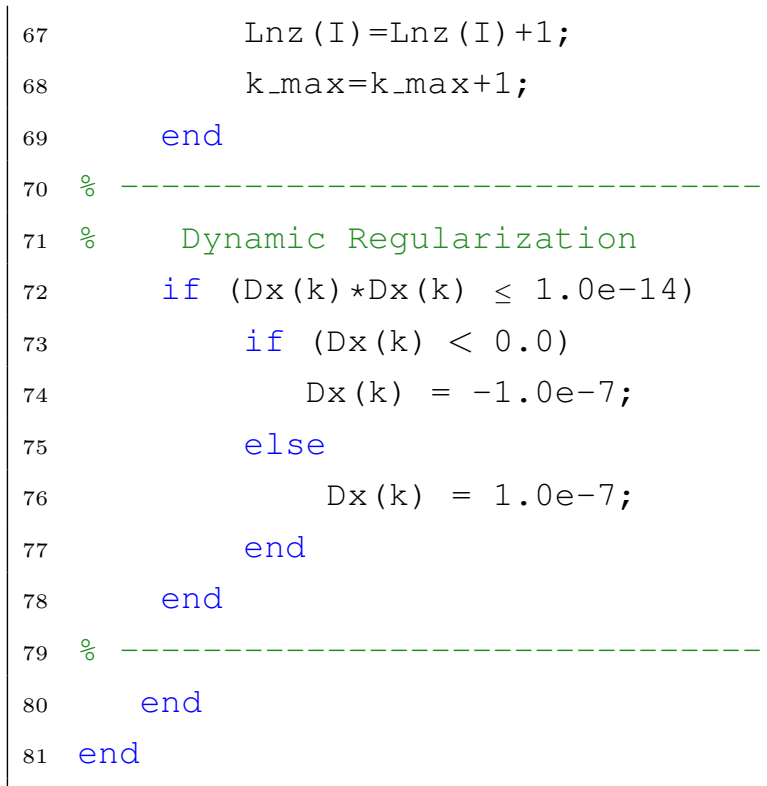

\subsection{Custom Solve Example: Second Approach}

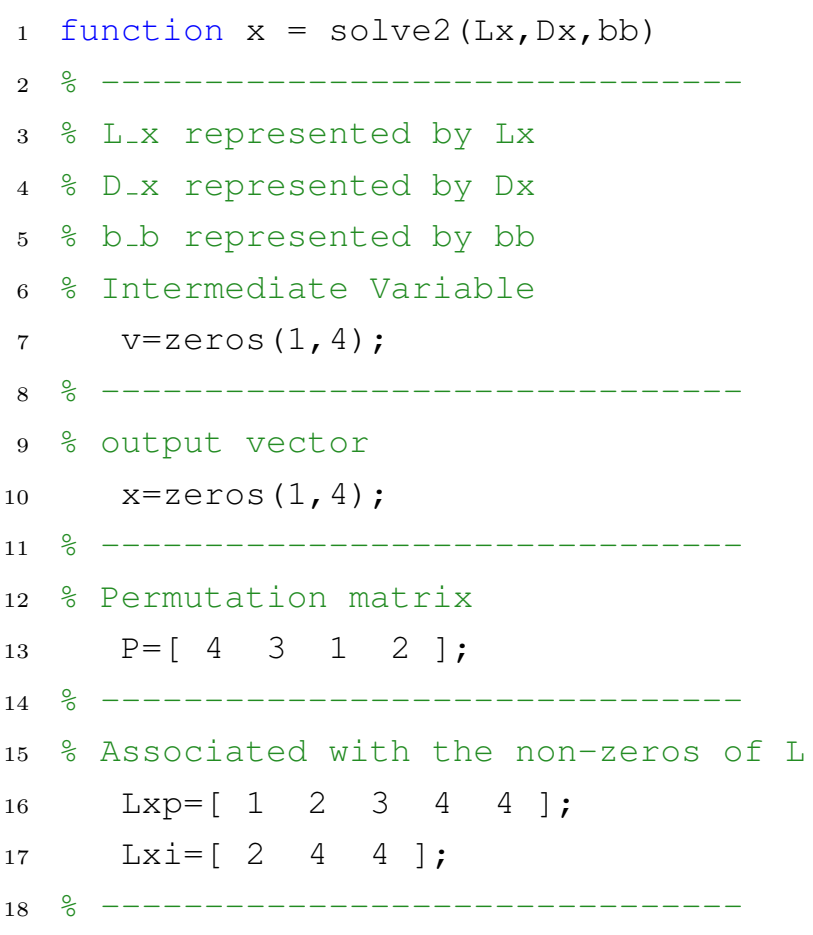




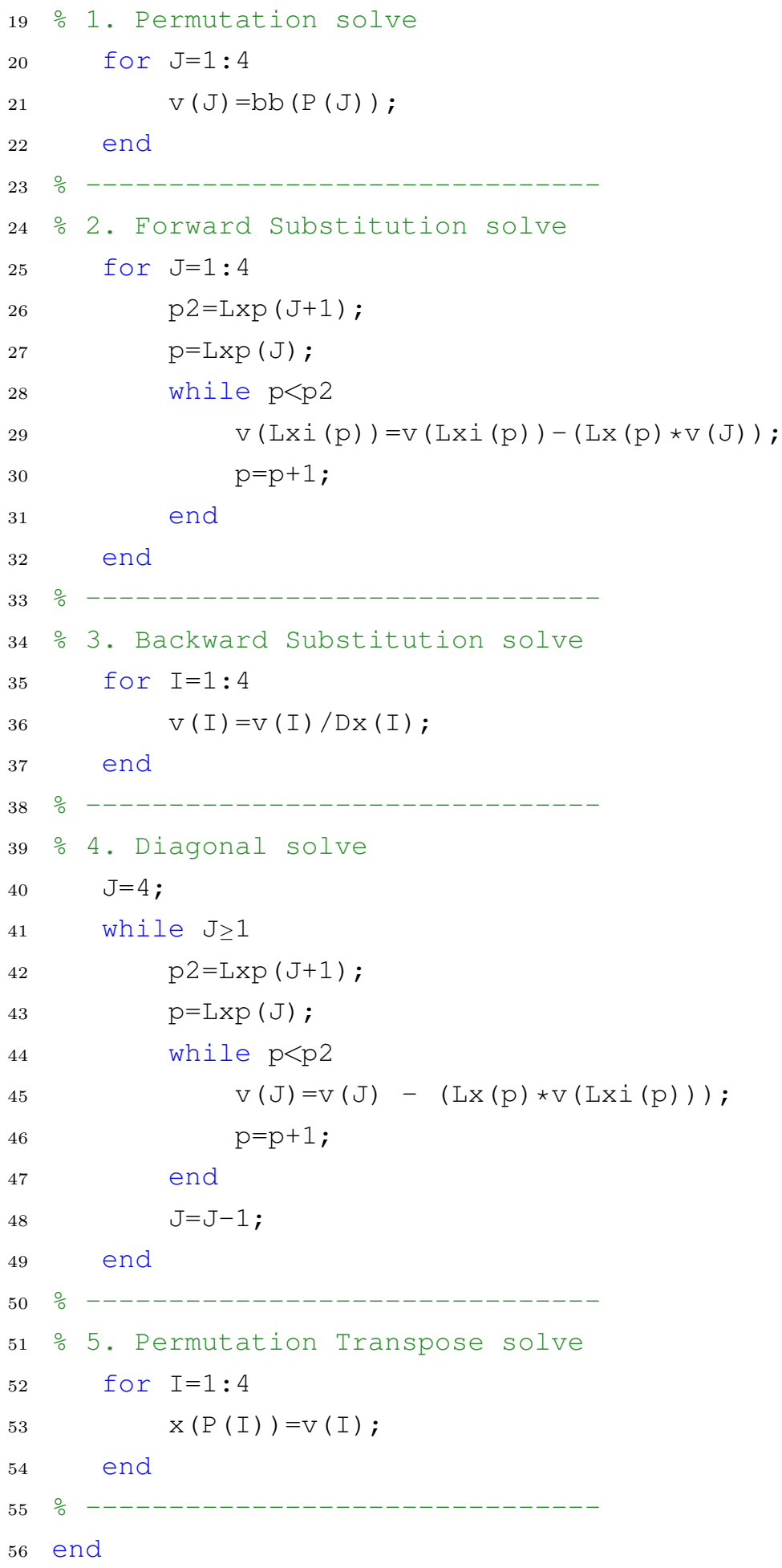




\section{Appendix 2}

\section{Bounds on the Kinematic Constraints}

$$
\begin{array}{lc}
\alpha: & \underline{\alpha} \leq a \leq \bar{\alpha} \\
\phi: & \underline{\phi} \leq \phi \leq \bar{\phi} \\
T: & 0 \leq \underline{T} \leq T \leq \bar{T}
\end{array}
$$

Given bounds (2.1), we need to find bounds on $U_{s}=\left(a, \omega_{\gamma}, \omega_{\psi}\right)$ such that bounds on $U_{s}$ satisfy $(2.2),(2.3)$, and (2.4).

$$
\begin{gathered}
T=M a+\frac{1}{2} \rho v^{2} S\left(C_{D_{0}}+K C_{L_{\alpha}}^{2} \alpha^{2}\right)+M g \sin (\gamma) \\
\phi=\arctan \left(\frac{v \cos (\gamma) \omega_{\psi}}{v \omega_{\gamma}+g \cos (\gamma)}\right) \\
\alpha=\left(\frac{M v \omega_{\gamma}+M g \cos (\gamma)}{\cos (\phi)\left(\frac{1}{2} \rho v^{2} S C_{L_{\alpha}}+T\right)}\right)
\end{gathered}
$$

Assumptions:

1. $\underline{T} \geq 0 N, \underline{\phi}=-\bar{\phi}, 0<\bar{\phi}<\frac{\pi}{2} \mathrm{rad}$, and $\bar{\alpha}>0 \mathrm{rad}$

2. $v>0 \mathrm{~m} / \mathrm{s}, \cos \gamma>0 \Longrightarrow\left(|\gamma|<\frac{\pi}{2} \mathrm{rad}\right.$ ), and $\cos \phi>0$ (by assumption 1).

\section{- Bounds on a:}

- Lower bounds $\underline{a}$ : From (2.2), we require 


$$
\underline{T} \leq M a+\frac{1}{2} \rho v^{2} S\left(C_{D_{0}}+K C_{L_{\alpha}}^{2} \alpha^{2}\right)+M g \sin (\gamma)
$$

So we want

$$
\frac{1}{M}\left[\underline{T}-\frac{1}{2} \rho v^{2} S\left(C_{D_{0}}+K C_{L_{\alpha}}^{2} \alpha^{2}\right)-M g \sin (\gamma)\right] \leq a,
$$

To find lower bound on $a$, guaranteeing this, we maximize the left-hand-side of (2.6). There are two cases:

* Case 1: $\underline{\alpha} \leq 0 \mathrm{rad}$.

In this case, we maximize the left-hand-side by minimizing $\alpha$ which is set to 0 due to its quadratic nature. Thus, we set

$$
\underline{a}=\frac{1}{M}\left[\underline{T}-\frac{1}{2} \rho v^{2} S C_{D_{0}}-M g \sin (\gamma)\right]
$$

Check: $\underline{a} \leq a \Longrightarrow \frac{1}{M}\left[\underline{T}-\frac{1}{2} \rho v^{2} S C_{D_{0}}-M g \sin (\gamma)\right] \leq a$

$$
\Longrightarrow \underline{T} \leq M a+\frac{1}{2} \rho v^{2} S C_{D_{0}}+M g \sin (\gamma) \leq T
$$

* Case 2: $\underline{\alpha}>0 \mathrm{rad}$.

In this case, we maximize the left-hand-side by setting the minimum value of $\alpha$, which is $\underline{\alpha}>0$, and set

$$
\underline{a}=\frac{1}{M}\left[\underline{T}-\frac{1}{2} \rho v^{2} S\left(C_{D_{0}}+K C_{L_{\alpha}}^{2} \underline{\alpha}^{2}\right)-M g \sin (\gamma)\right]
$$

Check: $\underline{a} \leq a \Longrightarrow \frac{1}{M}\left[\underline{T}-\frac{1}{2} \rho v^{2} S\left(C_{D_{0}}+K C_{L_{\alpha}}^{2} \underline{\alpha}^{2}\right)-M g \sin (\gamma)\right] \leq a$

$\Longrightarrow \underline{T} \leq M a+\frac{1}{2} \rho v^{2} S\left(C_{D_{0}}+K C_{L_{\alpha}}^{2} \underline{\alpha}^{2}\right)+M g \sin (\gamma) \leq T$

- Upper bounds $\bar{a}$ : Again from (2.2), we require

$$
\bar{T} \geq M a+\frac{1}{2} \rho v^{2} S\left(C_{D_{0}}+K C_{L_{\alpha}}^{2} \alpha^{2}\right)+M g \sin (\gamma)
$$


So we want,

$$
a \leq \frac{1}{M}\left[\bar{T}-\frac{1}{2} \rho v^{2} S\left(C_{D_{0}}+K C_{L_{\alpha}}^{2} \alpha^{2}\right)-M g \sin (\gamma)\right],
$$

To find an upper-bound on $a$, we minimize the right-hand-side of (2.10). Since $\bar{\alpha}>0 \mathrm{rad}$, we use the maximum value of $\alpha$ and set,

$$
\bar{a}=\frac{1}{M}\left[\bar{T}-\frac{1}{2} \rho v^{2} S\left(C_{D_{0}}+K C_{L_{\alpha}}^{2} \bar{\alpha}^{2}\right)-M g \sin (\gamma)\right] .
$$

Check: $\bar{a} \geq a \Longrightarrow \frac{1}{M}\left[\bar{T}-\frac{1}{2} \rho v^{2} S\left(C_{D_{0}}+K C_{L_{\alpha}}^{2} \bar{\alpha}^{2}\right)-M g \sin (\gamma)\right] \geq a$

$$
\Longrightarrow \bar{T} \geq M a+\frac{1}{2} \rho v^{2} S\left(C_{D_{0}}+K C_{L_{\alpha}}^{2} \bar{\alpha}^{2}\right)+M g \sin (\gamma) \geq T
$$

These guarantee $\underline{a} \leq a \leq \bar{a} \Longrightarrow \underline{T} \leq T \leq \bar{T}$.

- Thus to summarize:

$$
\begin{gathered}
\underline{a}=\left\{\begin{array}{cc}
\frac{1}{M}\left[\underline{T}-\frac{1}{2} \rho v^{2} S C_{D_{0}}-M g \sin (\gamma)\right], & \underline{\alpha} \leq 0 \\
\frac{1}{M}\left[\underline{T}-\frac{1}{2} \rho v^{2} S\left(C_{D_{0}}+K C_{L_{\alpha}}^{2} \underline{\alpha}^{2}\right)-M g \sin (\gamma)\right] & \underline{\alpha}>0
\end{array}\right. \\
\bar{a}=\frac{1}{M}\left[\bar{T}-\frac{1}{2} \rho v^{2} S\left(C_{D_{0}}+K C_{L_{\alpha}}^{2} \bar{\alpha}^{2}\right)-M g \sin (\gamma)\right] .
\end{gathered}
$$

\section{- Bounds on $\omega_{\gamma}$ :}

- Lower bounds $\underline{\omega_{\gamma}}$ : First, by assumption 1, we require $|\phi| \leq \frac{\pi}{2} \mathrm{rad}$, so that by (2.3), we require

$$
\begin{gathered}
v \omega_{\gamma}+g \cos (\gamma)>0 \\
\Longrightarrow \omega_{\gamma}>-\frac{g \cos (\gamma)}{v}
\end{gathered}
$$

This is satisfied if 


$$
\underline{\omega_{\gamma}}=-\frac{g \cos (\gamma)}{v}+\epsilon_{c}, \text { for any } \epsilon_{c}>0
$$

Next, from (2.4), we want

$$
\underline{\alpha} \leq \frac{m v \omega_{\gamma}+m g \cos (\gamma)}{A}
$$

where $A=\cos (\phi)\left(\frac{1}{2} \rho v^{2} S C_{l_{\alpha}}+T\right)>0$ by assumption 1 and 2 .

So, we want

$$
\frac{\underline{\alpha} A-m g \cos (\gamma)}{m v} \leq \omega_{\gamma}
$$

To find a lower bound on $\omega_{\gamma}$, guaranteeing this, we maximize the left-hand-side of (2.16). There are two cases:

* Case 1: $\underline{\alpha}<0 \mathrm{rad}$. In this case, we minimize $A$ and set

$$
\underline{\omega_{\gamma}}=\frac{\underline{\alpha} \cos (\bar{\phi})\left(\frac{1}{2} \rho v^{2} S C_{l_{\alpha}}+\underline{T}\right)-m g \cos (\gamma)}{m v} .
$$

The assumption $\underline{\phi}=-\bar{\phi}$ has been used.

Note that

$$
\begin{gathered}
\cos (\bar{\phi})\left(\frac{1}{2} \rho v^{2} S C_{l_{\alpha}}+\underline{T}\right) \leq A \\
\Longrightarrow \underline{\alpha} \cos (\bar{\phi})\left(\frac{1}{2} \rho v^{2} S C_{l_{\alpha}}+\underline{T}\right) \geq \underline{\alpha} A .
\end{gathered}
$$

We can also check if $\omega_{\gamma} \geq \underline{\omega_{\gamma}} \Longrightarrow \alpha \geq \underline{\alpha}$

$$
\begin{gathered}
\text { Since, } \frac{\underline{\alpha} A-m g \cos (\gamma)}{m v} \leq \frac{\underline{\alpha} \cos (\bar{\phi})\left(\frac{1}{2} \rho v^{2} S C_{L_{\alpha}}+\underline{T}\right)-m g \cos (\gamma)}{m v}=\underline{\omega_{\gamma}} \leq \omega_{\gamma} \\
\Longrightarrow \underline{\alpha} \leq \frac{m v \omega_{\gamma}+m g \cos (\gamma)}{A}=\alpha, \text { as required }
\end{gathered}
$$


* Case 2: $\underline{\alpha} \geq 0 \mathrm{rad}$. In this case, we maximize $A$ and set

$$
\underline{\omega_{\gamma}}=\frac{\alpha\left(\frac{1}{2} \rho v^{2} S C_{l_{\alpha}}+\bar{T}\right)-m g \cos (\gamma)}{m v} .
$$

Note that we now have $\underline{\alpha} A \leq \underline{\alpha}\left(\frac{1}{2} \rho v^{2} S C_{l_{\alpha}}+\bar{T}\right)$. Again we can check if $\omega_{\gamma} \geq$ $\underline{\omega_{\gamma}} \Longrightarrow \alpha \geq \underline{\alpha}$. It follows the same procedure as above.

$$
\begin{gathered}
\frac{\underline{\alpha} A-m g \cos (\gamma)}{m v} \leq \frac{\underline{\alpha}\left(\frac{1}{2} \rho v^{2} S C_{L_{\alpha}}+\bar{T}\right)-m g \cos (\gamma)}{m v}=\underline{\omega_{\gamma}} \leq \omega_{\gamma} \\
\Longrightarrow \underline{\alpha} \leq \frac{m v \omega_{\gamma}+m g \cos (\gamma)}{A}=\alpha .
\end{gathered}
$$

- So, given (2.14), (2.17) and (2.18), we take $\omega_{\gamma}$ as follows.

* Case 1: $\underline{\alpha}<0$ rad.

From (2.17),

$$
\underline{\omega_{\gamma}}=\frac{\underline{\alpha} \cos (\bar{\phi})\left(\frac{1}{2} \rho v^{2} S C_{L_{\alpha}}+\underline{T}\right)}{m v}-\frac{g \cos (\gamma)}{v}
$$

where

$$
\frac{\underline{\alpha} \cos (\bar{\phi})\left(\frac{1}{2} \rho v^{2} S C_{L_{\alpha}}+\underline{T}\right)}{m v}<0 .
$$

Thus, since (2.14) also needs to be satisfied, we take

$$
\underline{\omega_{\gamma}}=-\frac{g \cos (\gamma)}{v}+\epsilon_{c}, \text { for some } \epsilon_{c} \dot{\iota} 0 \text {. }
$$

* Case 2: $\underline{\alpha} \geq 0 \mathrm{rad}$.

From (2.18),

$$
\underline{\omega_{\gamma}}=\frac{\underline{\alpha}\left(\frac{1}{2} \rho v^{2} S C_{L_{\alpha}}+\bar{T}\right)}{m v}-\frac{g \cos (\gamma)}{v}
$$

where 


$$
\frac{\underline{\alpha}\left(\frac{1}{2} \rho v^{2} S C_{L_{\alpha}}+\bar{T}\right)}{m v} \geq 0
$$

If $\underline{\alpha}>0 \mathrm{rad}$, we take (2.20). Otherwise, if $\underline{\alpha}=0 \mathrm{rad}$, we take (2.19).

- Upper bound $\bar{\omega}_{\gamma}$ : From (2.4), we want

$$
\begin{aligned}
& \bar{\alpha} \geq \frac{m v \omega_{\gamma}+m g \cos (\gamma)}{A} \\
& \Longrightarrow \omega_{\gamma} \leq \frac{\bar{\alpha} A-m g \cos (\gamma)}{m v}
\end{aligned}
$$

To find an upper-bound on $\omega_{\gamma}$, we minimize the right-hand-side of (2.21). Since $\bar{\alpha}>0 \mathrm{rad}$ (by assumption 1 ), we minimize $A$. Hence, we set

$$
\bar{\omega}_{\gamma} \leq \frac{\bar{\alpha} \cos (\bar{\phi})\left(\frac{1}{2} \rho v^{2} S C_{L_{\alpha}}+\underline{T}\right)-m g \cos (\gamma)}{m v}
$$

Check:

$$
\text { Since, } \begin{gathered}
\frac{\bar{\alpha} A-m g \cos (\gamma)}{m v} \geq \frac{\bar{\alpha} \cos (\bar{\phi})\left(\frac{1}{2} \rho v^{2} S C_{L_{\alpha}}+\underline{T}\right)-m g \cos (\gamma)}{m v}=\bar{\omega}_{\gamma} \geq \omega_{\gamma} \\
\Longrightarrow \bar{\alpha} \geq \frac{m v \omega_{\gamma}+m g \cos (\gamma)}{A}=\alpha
\end{gathered}
$$

as required.

- Thus to summarize:

$$
\begin{aligned}
& \bar{\omega}_{\gamma}=\frac{\bar{\alpha} \cos (\bar{\phi})\left(\frac{1}{2} \rho v^{2} S C_{L_{\alpha}}+\underline{T}\right)-m g \cos (\gamma)}{m v} \\
& \underline{\omega_{\gamma}}=\left\{\begin{array}{cl}
-\frac{g \cos (\gamma)}{v}+\epsilon_{c}, & \underline{\alpha} \leq 0 \\
\frac{\alpha\left(\frac{1}{2} \rho v^{2} S C_{L_{\alpha}}+\bar{T}\right)}{m v}-\frac{g \cos (\gamma)}{v} & \underline{\alpha}>0
\end{array} \text { where } \epsilon_{c}>0\right.
\end{aligned}
$$




\section{- Bounds on $\omega_{\psi}$ :}

- Lower bound $\underline{\omega_{\psi}}$ :

From (2.3), we want

$$
\tan (\underline{\phi}) \leq \frac{v \cos (\gamma) \omega_{\psi}}{v \omega_{\gamma}+g \cos (\gamma)}
$$

By the definition of $\underline{\omega_{\gamma}}$ above, $v \omega_{\gamma}+g \cos (\gamma)>0$

$$
\Longrightarrow \text { we want, } \omega_{\psi} \geq \frac{\tan (\underline{\phi})\left(v \omega_{\gamma}+g \cos (\gamma)\right)}{v \cos (\gamma)}
$$

To find $\omega_{\psi}$, we maximize the right-hand-side of (2.24).

By assumption $1, \tan (\underline{\phi})<0$. Hence, we minimize $v \omega_{\gamma}+g \cos (\gamma)$ and take

$$
\underline{\omega_{\psi}}=\frac{\tan (\underline{\phi})\left(v \underline{\omega_{\gamma}}+g \cos (\gamma)\right)}{v \cos (\gamma)}
$$

Check:

$$
\begin{gathered}
\frac{\tan (\underline{\phi})\left(v \omega_{\gamma}+g \cos (\gamma)\right)}{v \cos (\gamma)} \leq \frac{\tan (\underline{\phi})\left(v \underline{\omega_{\gamma}}+g \cos (\gamma)\right)}{v \cos (\gamma)}=\underline{\omega}_{\psi} \leq \omega_{\psi} \\
\Longrightarrow \tan (\underline{\phi}) \leq \frac{v \cos (\gamma) \omega_{\psi}}{v \omega_{\gamma}+g \cos (\gamma)}=\tan (\phi), \text { as required }
\end{gathered}
$$

- Upper bound $\bar{\omega}_{\psi}$ :

From (2.3), we want

$$
\begin{gathered}
\tan (\bar{\phi}) \geq \frac{v \cos (\gamma) \omega_{\psi}}{v \omega_{\gamma}+g \cos (\gamma)} \\
\Longrightarrow \frac{\tan (\bar{\phi})\left(v \omega_{\gamma}+g \cos (\gamma)\right)}{v \cos (\gamma)} \geq \omega_{\psi}
\end{gathered}
$$


To find $\bar{\omega}_{\psi}$, we minimize left-hand-side of (2.26). Take

$$
\bar{\omega}_{\psi}=\frac{\tan (\bar{\phi})\left(v \underline{\omega} \omega_{\gamma}+g \cos (\gamma)\right)}{v \cos (\gamma)}
$$

Check:

$$
\begin{gathered}
\frac{\tan (\bar{\phi})\left(v \omega_{\gamma}+g \cos (\gamma)\right)}{v \cos (\gamma)} \geq \frac{\tan (\bar{\phi})\left(v \underline{\omega_{\gamma}}+g \cos (\gamma)\right)}{v \cos (\gamma)}=\bar{\omega}_{\psi} \geq \omega_{\psi} \\
\Longrightarrow \tan (\bar{\phi}) \geq \frac{v \cos (\gamma) \omega_{\psi}}{v \omega_{\gamma}+g \cos (\gamma)}=\tan (\phi), \text { as required }
\end{gathered}
$$

- In summary:

$$
\begin{aligned}
\underline{\omega_{\psi}} & =\frac{\tan (\underline{\phi})\left(v \underline{\omega_{\gamma}}+g \cos (\gamma)\right)}{v \cos (\gamma)} \\
\bar{\omega}_{\psi} & =\frac{\tan (\bar{\phi})\left(v \underline{\omega_{\gamma}}+g \cos (\gamma)\right)}{v \cos (\gamma)}
\end{aligned}
$$

Note: By assumption 1, $\underline{\phi}=-\bar{\phi}$, so $\underline{\omega_{\psi}}=-\bar{\omega}_{\psi}$, which is correct. 


\section{Appendix 3}

\section{A Brief Note on the Convexification}

\section{Cost Function}

Given the continuous cost function $J(Z)=\int_{t}^{t+H_{p}} F(Z) d t$, consider $\tilde{Z}^{0}=\left(X_{t}^{0}, U_{t}^{0}, X_{t+\Delta t}^{0}, U_{t+\Delta t}^{0}, \ldots, U_{t+(N-1) \Delta t}^{0}, X_{t+N \Delta t}^{0}\right)$ are the discrete nominal points around which we will approximate the cost function by the second order Taylor approximation and

$\tilde{Z}=\left(X_{t}, U_{t}, X_{t+\Delta t}, U_{t+\Delta t}, \ldots, U_{t+(N-1) \Delta t}, X_{t+N \Delta t}\right)$ are the predictive solution except $X_{t}$ ( $X_{t}$ is the current state), $\tau \in\{t, t+\Delta t, \ldots, t+N \Delta t\}$ and $N$ is the number of predictive points. Then, the discrete cost approximation can be written as

$$
J(\tilde{Z}) \approx F\left(\tilde{Z}^{0}\right)+F^{\prime}\left(\tilde{Z}^{0}\right)\left(\tilde{Z}-\tilde{Z}^{0}\right)+\frac{1}{2 !} F^{\prime \prime}\left(\tilde{Z}^{0}\right)\left(\tilde{Z}-\tilde{Z}^{0}\right)^{2}+H . O \cdot T
$$

The approximate cost (3.1) after neglecting Higher Order Terms (H.O.T), it can be written as,

$$
\tilde{J}(\tilde{Z})=\frac{1}{2}\left(\tilde{Z}-\tilde{Z}^{0}\right)^{T} H\left(\tilde{Z}^{0}\right)\left(\tilde{Z}-\tilde{Z}^{0}\right)+R\left(\tilde{Z}^{0}\right)^{T}\left(\tilde{Z}-\tilde{Z}^{0}\right)+F\left(\tilde{Z}^{0}\right)
$$

where, $H\left(\tilde{Z}^{0}\right)=F^{\prime \prime}\left(\tilde{Z}^{0}\right)$ refers to the Hessian and $R\left(\tilde{Z}^{0}\right)=F^{\prime}\left(\tilde{Z}^{0}\right)$ refers to the gradient. 


\section{System Equation}

Given the system dynamics as $\dot{X}(\tau)=f(X(\tau), U(\tau))$, it can be approximated by the first order Taylor approximation at the discrete nominal point $\left(X_{\tau}^{0}, U_{\tau}^{0}\right) \in \tilde{Z}^{0}$ as,

$$
\left.\dot{X}(\tau) \approx f(X(\tau), U(\tau))\right|_{\left(X_{\tau}^{0}, U_{\tau}^{0}\right)}+\left.f^{\prime}(X(\tau), U(\tau))\right|_{\left(X_{\tau}^{0}, U_{\tau}^{0}\right)}\left(\begin{array}{c}
X(\tau)-X_{\tau}^{0} \\
U(\tau)-U_{\tau}^{0}
\end{array}\right)+\text { H.O.T }
$$

The linearized system equation (3.3) after neglecting Higher Order Terms, we write as,

$$
\dot{X}(\tau)=\left.f(X(\tau), U(\tau))\right|_{\left(X_{\tau}^{0}, U_{\tau}^{0}\right)}+A_{c}(\tau)\left(X(\tau)-X_{\tau}^{0}\right)+B_{c}(\tau)\left(U(\tau)-U_{\tau}^{0}\right)
$$

which can be further written as,

$$
\dot{X}(\tau)=A_{c}(\tau) X(\tau)+B_{c}(\tau) U(\tau)+g_{c}(\tau)
$$

where,

$$
\begin{gathered}
A_{c}(\tau):=\left.\frac{\partial f}{\partial X(\tau)}\right|_{\left(X_{\tau}^{0}, U_{\tau}^{0}\right)} \\
B_{c}(\tau):=\left.\frac{\partial f}{\partial U(\tau)}\right|_{\left(X_{\tau}^{0}, U_{\tau}^{0}\right)} \\
g_{c}(\tau):=\left.f(X(\tau), U(\tau))\right|_{\left(X_{\tau}^{0}, U_{\tau}^{0}\right)}-A_{c}(\tau) X_{\tau}^{0}-B_{c}(\tau) U_{\tau}^{0}
\end{gathered}
$$

Since equilibrium point is not set, above problem is affine. We get discrete affine system using sampling time $\Delta t$ as [117],

$$
X(\tau+\Delta t)=A_{d}^{\tau} X(\tau)+B_{d}^{\tau} U(\tau)+g_{d}^{\tau}
$$

where $\tau \in\{t, t+\Delta t, \ldots, t+N \Delta t\}$ and the discrete matrix can be computed as, 


$$
\exp \left(\begin{array}{c|ccc}
A_{c}(\tau) \cdot \Delta t & B_{c}(\tau) \cdot \Delta t & & g_{c}(\tau) \cdot \Delta t \\
0 & & 0 &
\end{array}\right)=\left(\begin{array}{c|ccc}
A_{d}^{\tau} & B_{d}^{\tau} & & g_{d}^{\tau} \\
0 & & 1
\end{array}\right)
$$




\section{Appendix 4}

\section{Quad-rotor Characteristics and Problems Parameters}

\subsection{Quad-rotor Characteristics}

\begin{tabular}{|c|c|c|c|}
\hline 1 & \multicolumn{3}{|c|}{ 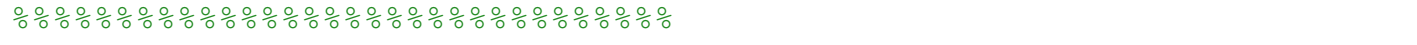 } \\
\hline 2 & \multicolumn{3}{|c|}{$\%$ Quadcopter Characteristics $\%$} \\
\hline 3 & \multicolumn{3}{|c|}{ 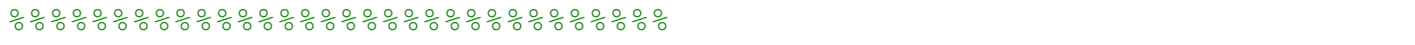 } \\
\hline 4 & 9 & $=9.81 ;$ & Acceleration due to gravity \\
\hline 5 & m & $=0.468 ;$ & Mass of the quadcopter \\
\hline 6 & $\mathrm{~L}$ & $=0.225 ;$ & Half the distance between two opposite motor \\
\hline 7 & $\mathrm{k}$ & $=1.14 * 1 \mathrm{e}-7$; & Lift constant \\
\hline 8 & $\mathrm{~b}$ & $=2.98 * 1 e-6$ & Drag constant \\
\hline 9 & Ix & $=4.856 * 1 e-3 ;$ & Moment of Inertia along $x$-axis \\
\hline 10 & IY & $=4.856 * 1 e-3 ;$ & Moment of Inertia along $y$-axis \\
\hline 1 & Iz & $=8.801 * 1 e-3 ;$ & Moment of Inertia along z-axis \\
\hline 2 & $\mathrm{Kdx}$ & $=0.25$ & Drag force coefficient $x$-axis \\
\hline 13 & Kdy & $=0.25 ;$ & Drag force coefficient y-axis \\
\hline 14 & $\mathrm{Kdz}$ & $=0.25 ;$ & Drag force coefficient $z$-axis \\
\hline
\end{tabular}

\subsection{Bounds on Optimization Variables}




\begin{tabular}{|c|c|c|c|c|}
\hline \multicolumn{5}{|c|}{ 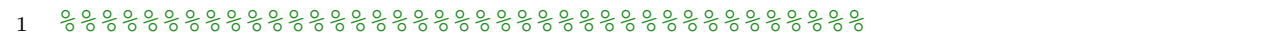 } \\
\hline \multicolumn{5}{|c|}{2 응 Bounds On Optimization Parameters $\frac{\circ}{0}$} \\
\hline 3 & \multicolumn{4}{|c|}{ 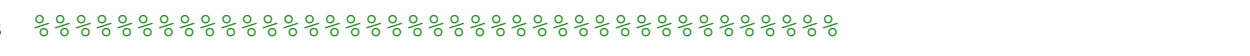 } \\
\hline 4 & \multirow{2}{*}{\multicolumn{4}{|c|}{$\begin{array}{l}\text { suffix: L-Lower bounds, U-Upper bounds } \\
\frac{\circ}{0} \circ \text { Bounds on States Variables } \frac{0}{0}\end{array}$}} \\
\hline 5 & & & & \\
\hline 6 & $\mathrm{xL}$ & $=-1$ & $x U=350$ & $\mathrm{x}-1+2$ \\
\hline 7 & VxL & $=-5$; & $\mathrm{VxU}=5 ;$ & velocity along $x$ \\
\hline 8 & $\mathrm{yL}$ & $=-50$; & $\mathrm{yU}=350$ & $\mathrm{y}$ \\
\hline 9 & VyL & $=-5$; & $\mathrm{VyU}=5 ;$ & velocity along y \\
\hline 10 & zL & $=0 ;$ & $\mathrm{zU}=150$ & $\mathrm{z}$ \\
\hline 11 & VzL & $=-5$; & $\mathrm{VzU}=5$ & velocity along $\mathrm{z}$ \\
\hline 12 & psiL $=$ & $=-p i / 3 ;$ & $\mathrm{psiU}=\mathrm{pi} / 3$ & roll angle \\
\hline 13 & O_phiL = & $=-p i / 6$ & O_phiU = pi/6; & rate of roll angle \\
\hline 14 & ThetaL & $=-p i / 3 ;$ & Thetau $=\mathrm{pi} / 3$; & pitch angle \\
\hline 15 & O_TheL $=$ & $=-\mathrm{pi} / 6 ;$ & O_TheU $=\mathrm{pi} / 6$; & rate of pitch angle \\
\hline 16 & KsiL $=$ & $=0$ & $\mathrm{KSiU}=0$ & yaw angle \\
\hline 17 & O_KsiL $=$ & $=0 ;$ & O_KsiU $=0$; & rate of pitch angle \\
\hline 18 & & & & \\
\hline 19 & $\div$ Boun & ids on Controls & Variables 응 & \\
\hline 20 & $\mathrm{~W} 1 \mathrm{~L}=$ & $=0 ; \quad W 1 \mathrm{U}$ & $=5000$ & RPM of Motor 1 \\
\hline 21 & W2L & $\mathrm{W} 2 \mathrm{U}$ & $=5000 ;$ & RPM of Motor 2 \\
\hline 22 & W3L & W3U & $=5000 ;$ & RPM of Motor 3 \\
\hline 23 & W4L & W4U & $=5000 ;$ & RPM of Motor 4 \\
\hline
\end{tabular}

\subsection{Bounds on Optimization Variables for Monte- Carlo Test}

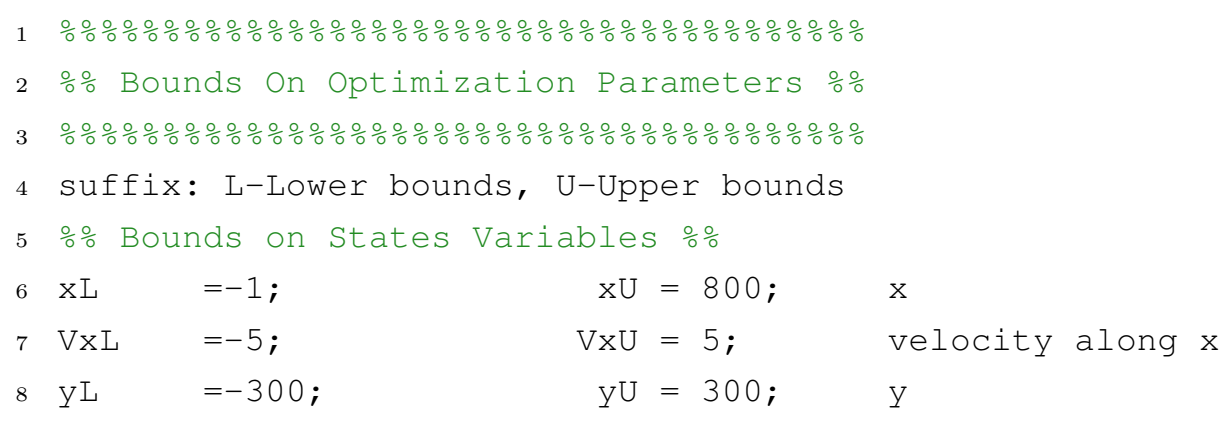




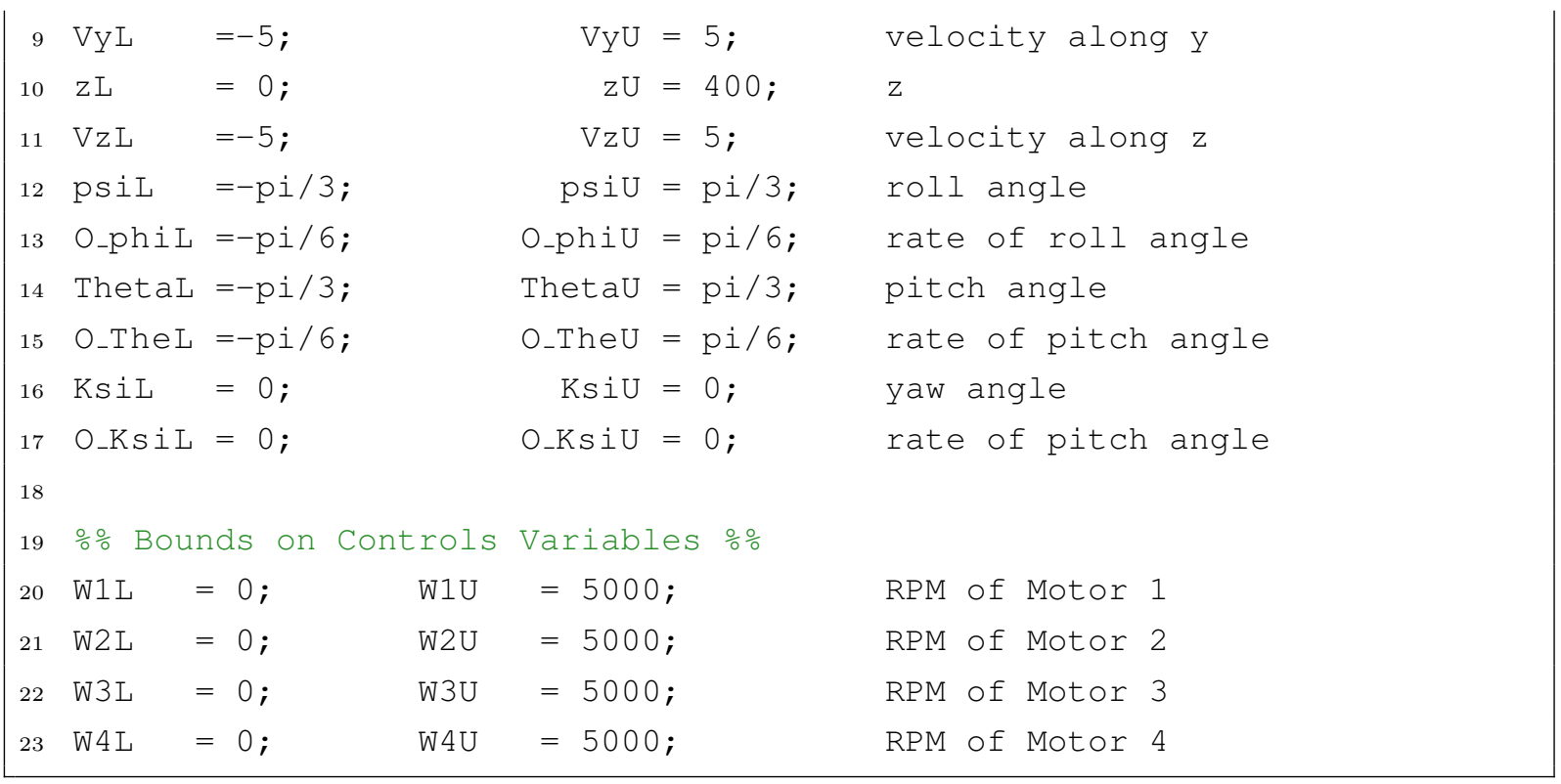

\subsection{Problem Scenario One}

\begin{tabular}{|cllllllllllll|}
\hline 1 & $x_{\{c, i\}}$ & $y_{\{c, i\}}$ & $z_{\{c, i\}}$ & $a_{i}$ & $b_{i}$ & $c_{i}$ & $p_{x_{i}}$ & $p_{y_{i}}$ & $p_{z_{i}}$ & $v_{x_{o}}$ & $v_{y_{o}}$ & $v_{z_{o}}$ \\
2 & 60 & 105 & 10 & 10 & 10 & 20 & 8 & 8 & 8 & 0 & 0 & 0 \\
3 & 60 & 155 & 10 & 10 & 10 & 20 & 8 & 8 & 8 & 0 & 0 & 0 \\
4 & 50 & 205 & 10 & 10 & 10 & 20 & 8 & 8 & 8 & 0 & 0 & 0 \\
5 & 150 & 130 & 10 & 10 & 10 & 30 & 8 & 8 & 8 & 0 & 0 & 0 \\
6 & 170 & 180 & 10 & 10 & 10 & 30 & 8 & 8 & 8 & 0 & 0 & 0 \\
7 & 100 & 300 & 20 & 5 & 25 & 5 & 2 & 2 & 2 & 0 & -7.25 & 0 \\
8 & 130 & 0 & 20 & 5 & 25 & 5 & 2 & 2 & 2 & 0 & 6 & 0 \\
\hline
\end{tabular}

\subsection{Problem Scenario Two}

\begin{tabular}{|lllllllllllll|}
\hline 1 & $x_{\{c, i\}}$ & $y_{\{c, i\}}$ & $z_{\{c, i\}}$ & $a_{i}$ & $b_{i}$ & $c_{i}$ & $p_{x_{i}}$ & $p_{y_{i}}$ & $p_{z_{i}}$ & $v_{x_{o}}$ & $v_{y_{o}}$ & $v_{z_{o}}$ \\
2 & 40 & 55 & 10 & 10 & 10 & 20 & 8 & 8 & 8 & 0 & 0 & 0 \\
3 & 60 & 105 & 10 & 10 & 10 & 20 & 8 & 8 & 8 & 0 & 0 & 0 \\
4 & 60 & 155 & 10 & 10 & 10 & 20 & 8 & 8 & 8 & 0 & 0 & 0
\end{tabular}




\subsection{PROBLEM SCENARIO TWO}

\begin{tabular}{|lllllllllllll}
5 & 50 & 205 & 10 & 10 & 10 & 20 & 8 & 8 & 8 & 0 & 0 & 0 \\
6 & 70 & 255 & 10 & 10 & 10 & 20 & 8 & 8 & 8 & 0 & 0 & 0 \\
7 & 150 & 105 & 10 & 10 & 10 & 30 & 8 & 8 & 8 & 0 & 0 & 0 \\
8 & 170 & 205 & 10 & 10 & 10 & 30 & 8 & 8 & 8 & 0 & 0 & 0 \\
9 & 100 & 300 & 20 & 5 & 25 & 5 & 2 & 2 & 2 & 0 & -5 & 0 \\
10 & 130 & 0 & 20 & 5 & 25 & 5 & 2 & 2 & 2 & 0 & 3 & 0
\end{tabular}




\section{References}

[1] Michael S. Selig. Modeling full-envelope aerodynamics of small uavs in realtime. In AIAA Atmospheric Flight Mechanics 2010 Conference, Toronto, Ontario, Canada, August 2010. American Institute of Aeronautics and Astronautics. Inc.

[2] Chang bae Moon and Woojin Chung. Kinodynamic planner dual tree rrt (dt-rrt) for two-wheeled mobile robots using the rapidly exploring random tree. IEEE TRANSACTIONS ON INDUSTRIAL ELECTRONICS, 62(2):1080-1090, February 2015 .

[3] S. Owlia and A. de Ruiter. Autonomous obstacle avoidance for fixed-wing unmanned aerial vehicles. The Aeronautical Journal, 119(1221):1415-1436, November 2015.

[4] L. P. R. Lewis. Rapid motion planning and autonomous obstacles avoidance for unmanned vehicles. PhD thesis, Naval Postgraduate School, Dec 2006.

[5] Takeo K., Chuck T., and William W. Autonomous land vehicle project at cmu. Proceedings of the 1986 ACM fourteenth annual conference on Computer Science, Cincinnati, Ohio, USA, pages 71-80, 1986.

[6] Richard S. Wallace, Anthony Stentz, Charles E. Thorpe, Hans P. Moravec, William Whittaker, and Takeo Kanade. First results in robot road-following. JCAI'85 Proceedings of the 9th international joint conference on Artificial Intelligence, 1985.

[7] David Hyunchul Shim, Hoam Chung, H. Jin Kim, and Sastry Shankar. Autonomous exploration in unknown urban environments for unmanned aerial vehicles. AIAA Guidance, Navigation and Control Conference and Exhibit, San Francisco, California, 8:6381-6388, 15-18, August 2005. 
[8] LaValle S. M. Rapidly-Exploring Random Trees: A New Tool for Path Planning. $\mathrm{PhD}$ thesis, Iowa State University, Computer Science Department, Ames, Iowa., Oct. 1998.

[9] Amin J. N., Boskovic J. D., and Mehra R. K. A fast and efficient approach to path planning for unmanned vehicles. AIAA Guidance, Navigation and Control Conference and Exhibit, Keystone, Colorado, 21-24 August 2006.

[10] Steven M. Lavalle, James J. Kuffner, and Jr. Rapidly-Exploring Random Trees: Progress and Prospects. Algorithmic and Computational Robotics: New Directions. A K Peters, Wellesley, MA, $1^{\text {st }}$ edition, 2000.

[11] Kuffner J. J and LaValle S. M. Rrt-connect: An efficient approach to singlequery path planning. Proceedings of the International Conference on Robotics and Automation, IEEE, San Francisco, CA, 2:995-1001, 24-28 April 2000.

[12] Frazzoli E., Dahleh M. A., and Feron E. Real-time motion planning for agile autonomous vehicles. Journal of Guidance Control Dynamics, 25(1):116-129, 2002.

[13] S.S. GE and Y.J. CUI. Dynamic motion planning for mobile robots using potential field method. Autonomous Robots, 13, (3):207-222, November 2002.

[14] Chang D. E. and Marsden J. E. Gyroscopic forces and collision avoidance with convex obstacles. New Trends in Nonlinear Dynamics and Control and their Applications, LNCIS, 295:145-159, May 2004.

[15] Vissiere D. Guidance, Navigation and Control Solutions for Unmanned Heterogenous Vehicles in a Collaborative Mission. PhD thesis, MINES ParisTech, Paris, France, 2008.

[16] Vissiere D., Chang D. E., and Petit N. Experiments of trajectory generation and obstacle avoidance for a ugv. Proceedings of the 2007 American Control Conference, New York City, USA, pages 2828-2835, 9-13 July 2007.

[17] Bellman R. E. Dynamic Programming. PhD thesis, Princeton University, Princetion University Press, 1957. 
[18] Jr. Arthur E. Bryson and Yu-Chi Ho. Applied Optimal Control. Hemisphere Publishing Corporation, Washington, DC, 1975. ISBN-13: 978-0891162285, ISBN10: 9780891162285 .

[19] Stryk O. V. and Bulirsch R. Direct and indirect methods for trajectory optimization. Annual Operational Research, 37:335-354, 1992.

[20] I. M. Ross and Fariba Fahroo. Legendre Pseudospectral Approximations of Optimal Control Problems. Springer-Verlag, Lecture Notes, 2003.

[21] P. Falcone, M. Tufo, F. Borrelli, J. Asgari, and H.E. Tseng. A linear time varying model predictive control approach to the integrated vehicle dynamics control problem in autonomous systems. In Proceedings of the 46th IEEE Conference on Decision and Control, New Orleans, LA, USA, Dec. 2007.

[22] Philip E. Gill, Walter Murray, and Michael A. Saunders. Snopt: An sqp algorithm for large-scale constrained optimization. Society for Industrial and Applied Mathematics, 47(1):99-131, 2005.

[23] I. M. Ross. A begineer's guide to dido. A MATLAB Application Package for Solving Optimal Control Problems (Ver. 7.3), Elissar, LLC., 2007.

[24] Daniel Morgan, Soon-Jo Chung, and Fred Y. Hadaegh. Model predictive control of swarms of spacecraft using sequential convex programming. Journal of Guidance, Control, and Dynamics, 37(6), November-December 2014.

[25] M. A. Hurni, P. Sekhavat, and I. M. Ross. Autonomous trajectory planning using real-time information updates. AIAA Guidance, Navigation and Control Conference and Exhibit, Honolulu, Hawaii, August 18-21 2008.

[26] K. P. Bollino. High-fidelity real-time trajectory optimization for reusable launch vehicles. PhD thesis, Naval Postgraduate School, 2006.

[27] K. P. Bollino and L. R. Lewis. Collision-free multi-uav optimal path planning and cooperative control for tactical applications. AIAA Guidance, Navigation and Control, Honolulu, Hawaii, August 18-21 2008. 
[28] I. M. Ross and F. Fahroo. Issues in the real-time computation of optimal control. Mathematical and Computer Modelling, ELSEVIER, 43:1172-1188, May 42006.

[29] Camacho E. F. and Bordons C. Model predictive control. Springer, New York, 1996.

[30] Mayne D. Q., Rawlings J. B., Rao C. V., and Scokaert P. O. M. Constrained model predictive control: Stability and optimality. Automatica, 36(6):789-814, June 2000.

[31] Schittkowski K. and Zillober C. Sequential Convex Programming Methods. In: Marti K., Kall P. (eds) Stochastic Programming., volume 423, pp 123-141 of Lecture Notes in Economics and Mathematical Systems. Springer, Heidelberg, 1995. ISBN: 978-3-642-88272-2.

[32] Richards H. Bryd, Jean Charles Gilbert, and Jorge Nocedal. A trust region method based on interior point techniques for nonlinear programming. Mathematical Programming, 89(1):149-185, November 2000.

[33] Shim D. H. and Sastry S. An evasive maneuvering algorithm for uavs in see-andavoid situations. Proceedings of the 2007 American Control Conference, New York city, USA, pages 27-33, 9-13 July 2007.

[34] Wang X., Yadav V., and Balakrishanan S. N. Cooperative uav formation flying with obstacle/collision avoidance. IEEE Transactions on Control Systems Technology, 15(4):672-769, July 2007.

[35] Eirik Heggedal. Digital signal processing for uav wireless communication system. Thesis, Norwegian University of Science and Technology, June 2015.

[36] Bruce T. Clough. Metrics, schmetrics! how the heck do you determine a uav's autonomy anyway? August 2002. [Online: Accessed on 15 March 2019].

[37] Eric Kalvins and Valery Zagursky. Unmanned aerial vehicle movement trajectory detection in open environment. In Procedia Computer Science, volume 104, pages 400-407. ELSEVIER, 2017.

[38] Luca De Filippis. Advanced Path Planning and Collision Avoidance Algorithms for UAVs. PhD thesis, Politecnico di Torino, May 2012. 
[39] Babak Salamat and Andrea M. Tonello. Stochastic trajectory generation using particle swarm optimization for quadrotor unmanned aerial vehicles (uavs). MDPI: Aerospace, 4(27), May 2017.

[40] Ross Allen and Marco Pavone. A real-time framework for kinodynamic planning with application to quadrotor obstacle avoidance. In AIAA Guidance, Navigation, and Control Conference, Invited Session: Advances in Guidance and Control of Unmanned Air Vehicles, San Diego, California, USA, 4-8 January 2016.

[41] Markus Hehn and Raffaello D'Andrea. Quadrocopter trajectory generation and control. In 18th IFAC World Congress, Milano (Italy), August 28 - September 2 2011.

[42] Markus Hehn and Raffaello D'Andrea. Real-time trajectory generation for interception maneuvers with quadrocopters. In 2012 IEEE/RSJ International Conference on Intelligent Robots and Systems, pages 4979-4984, Vilamoura, Portugal, 7-12 October 2012. IEEE.

[43] Federico Augugliaro, Angela P. Schoellig, and Raffaello D'Andrea. Generation of collision-free trajectories for a quadrocopter fleet: A sequential convex programming approach. In 2012 IEEE/RSJ International Conference on Intelligent Robots and Systems, pages 1917-1922, Vilamoura, Portugal, 7-12 October 2012. IEEE.

[44] Carlos E. Luis and Angela P. Schoellig. Trajectory generation for multiagent pointto-point transistions via distributed model predictive control. In IEEE Robotics and Automation Letters, volume 4 (2), pages 375-382. IEEE, April 2019.

[45] Fei Gao, William Wu, Yi Lin, and Shaojie Shen. Online safe trajectory generation for quadrotors using fast marching method and bernstein basis polynomial. In 2018 IEEE International Conference on Robotics and Automation (ICRA), Brisbane, QLD, Australia, 21-25 May 2018. IEEE.

[46] Wolfgang Honig, James A. Preiss, T.K. Satish Kumar, Gaurav S. Sukhatme, and Nora Ayanian. Trajectory planning for quadrotor swarms. IEEE TRANSACTIONS ON ROBOTICS, 34 (4):856-869, August 2018. 
[47] Alex Kushleyev, Daniel Mellinger, and Vijay Kumar. Towards a swarm of agile micro quadrotors. Autonomous Robots, 35(4):287-300, July 2013.

[48] Myoung-Chul Park, Sung-Mo Kang, Jae-Gyeong Lee, Gwi-Han Ko, Koog-Hwan Oh, Hyo-Sung Ahn, Young-Cheol Choi, and Ji-Hwan Son. Realization of distributed formation flying using a group of autonomous quadcopters and application to visual performance show. In IEEE Transportation Electrification Conference and Expo, Asia-Pacific (ITEC), Busan, Korea, June 1-4 2016.

[49] Andrew J. Barry. High-Speed Autonomous Obstacle Avoidance with Pushbroom Stereo. PhD thesis, MASSACHUSETTS INSTITUTE OF TECHNOLOGY, February 2016.

[50] Adam Bry, Charles Richter, Abraham Bachrach, and Nicholas Roy. Aggressive flight of fixed-wing and quadrotor aircraft in dense indoor environments. The International Journal of Robotics Research, 37(2):969-1002, 2015.

[51] Stephen Nuske, Sanjiban Choudhury, Sezal Jain, Andrew Chambers, Luke Yoder, Sebastian Scherer, Lyle Chamberlain, Hugh Cover, and Sanjiv Singh. Autonomous exploration and motion planning for an unmanned aerial vehicle navigating rivers. Journal of Field Robotics, 32(8):1141-1162, 2015.

[52] Giuseppe Loianno, Chris Brunner, Gary McGrath, and Vijay Kumar. Estimation, control, and planning for aggressive flight with a small quadrotor with a single camera and imu. IEEE ROBOTICS AND AUTOMATION LETTERS, 2(2), 2017.

[53] Michael James Campobasso. Leader-follower trajectory generation and tracking for quadrotor swarms. Master's thesis, Embry-Riddle Aeronautical University, Daytona Beach, Florida, April 2017.

[54] Axel Bürkle, Florian Segor, and Matthias Kollmann. Towards autonomous micro uav swarms. Journal of Intelligent Robotic Systems, 61 (1-4):339-353, January 2011.

[55] James A. Preiss, Wolfgang Honig, Gaurav S. Sukhatme, and Nora Ayanian. Crazyswarm: A large nano-quadcopter swarm. In IEEE International Conference on Robotics and Automation (ICRA), Singapore, May 29 - June 32017. 
[56] Daniel Morgan, Giri P Subramanian, Soon-Jo Chung, and Fred Y Hadaegh. Swarm assignment and trajectory optimization using variable-swarm, distributed auction assignment and sequential convex programming. The International Journal of Robotics Research, 35(10):1261-1285, 2016.

[57] Jacob Mattingley and Stephen Boyd. Cvxgen: a code generator for embedded convex optimization. Optimization and Engineering, 13:1-27, March 2012.

[58] Jacob Mattingley. Cvxgen: a code generator for embedded convex optimization [webpage], [Online: Accessed on 31 October 2017].

[59] Alexandar Domahidi, Eric Chu, and Stephen Boyd. Ecos: An socp solver for embedded systems. In 2013 European Control Conference, pages 3071-3076, Zurich, Switzerland, 17-19 July 2013. ECC.

[60] Alexandar Domahidi, A. Zgraggen, M. Zeilinger, M. Morari, and C. Jones. Efficient interior point methods for multistage problems arising in receding horizon control. In Conference on Decision and Control, pages 668-674, Maui, HI, USA, Dec. 2012.

[61] Fabian Ullmann. Fiordos: A matlab toolbox for c-code generation for first order methods. Master's thesis, ETH Zurich, 2011.

[62] Stephen Boyd and Lieven Vandenberghe. Convex Optimization. Cambridge University Press, 2009.

[63] Philip E. Gill and Elizabeth Wong. Methods for convex and general quadratic programming. Math. Prog. Comp., Springer, 7:71-112, 2015.

[64] Elizabeth Wong. Active-Set Methods for Quadratic Programming. PhD thesis, UNIVERSITY OF CALIFORNIA, SAN DIEGO, 2011.

[65] Vandenberghe L. The cvxopt linear and quadratic cone program solvers [online: http://www.seas.ucla.edu/ vandenbe/publications/coneprog.pdf]. [Accessed: 15 March 2017], March 2010.

[66] Michael A. Saunders. Solution of sparse rectangular systems using lsqr and craig. BIT Numerical Mathematics, 35 (4):588-604, December 1995. 
[67] P. R. Amestoy, T. A. Davis, and I. S. Duff. Algorithm 837: Amd, an approximate minimum degree ordering algorithm. ACM Transactions on Mathematical Software (TOMS), 30 (3):381-388, September 2004.

[68] Duff IS, Erisman AM, and Reid JK. Direct methods for sparse matrices. Oxford University Press, London, 1989.

[69] Timothy A. Davis. User guide for ldl, a concise sparse cholesky package. Version 2.0.3, January 252011.

[70] C. Goerzen, Z. Kong, and B. Mettler. A survey of motion planning algorithms from the perspective of autonomous uav guidance. J Intell Robot Syst, 57 (1-4):65-100, 2010.

[71] Walton Pereira Coutinho, Maria Battarra, and Jorg Fliege. The unmanned aerial vehicle routing and trajectory optimization problem, a taxonomic review. Computer and Industrial Engineering: ELSEVIER, 120:116-128, 2018.

[72] Yijing Zhao, Zheng Zheng, and Yang Liu. Survey on computational-intelligencebased uav path planning. Knowledge-Based Systems: ELSEVIER, 158:54-64, 15 October 2018.

[73] Weiran Yao, Naiming Qi, and Yanfang Liu. Online trajectory generation with rendezvous for uavs using multistage path prediction. J. Aerosp. Eng., 3(30), 2017.

[74] A. Albert, F. S. Leira, and L. Imsland. Uav path planning using milp with experiments. Norwegian Society of Automatic Control: Modeling, Identification and Control, 38(1):21-32, 2017.

[75] Subbarao Kambhampati and Larry S. Davis. Multiresolution path planning for mobile robots. IEEE JOURNAL OF ROBOTICS AND AUTOMATION, RA2(3):135-145, September 1986.

[76] P. Chandler, S. Rasmussen, and M. Pachter. Uav cooperative path planning. In AIAA Guidance, Navigation, and Control Conference, pages 1301-1306, Denver. CO, AIAA-2000-4370, August 14-17 2000. 
[77] O. Khatib. Real time obstacle avoidance for manipulators and mobile robots. In Proc. IEEE Int. Conf. on Robotics and Automation, pages 500-505, St. Louis, MO, USA, 25-28 March 1985. IEEE.

[78] Zhu Lihua, Cheng Xianghong, and Yuan Fuh-Gwo. A 3d collision avoidance strategy for uav with physical constraints. Measurement: ELSEVIER, 77:40-49, January 2016.

[79] M. Jun and R. D'Andrea. Path planning for unmanned aerial vehicles in uncertain and adversarial environments. In Cooperative Control: Models, Applications and Algorithms, volume 1, pages 95-110, 2003.

[80] Yucong Lin and Srikanth Saripalli. Sampling-based path planning for uav collision avoidance. IEEE TRANSACTIONS ON INTELLIGENT TRANSPORTATION SYSTEMS, 18(11), 2017.

[81] Hongxing Zheng, Jifeng Guo, and Peng Yan. A hybrid trajectory planning algorithm for uavs in cluttered environments. In 2018 9th International Conference on Mechanical and Aerospace Engineering (ICMAE), pages 389-393, Budapest, Hungary, 10-13 July 2018. IEEE.

[82] John T. Betts. Survey of numerical methods for trajectory optimization. JOURNAL OF GUIDANCE, CONTROL AND DYNAMICS, 21(2):192-207, March-April 1998.

[83] Ghulam Farid, Hongwei Mo, Muhammad Irshad Zahoor, and Qu Liwei. Computationally efficient algorithm to generate a waypoints-based-trajectory for a quadrotor uav. In 2018 Chinese Control And Decision Conference (CCDC), pages 4414-4419, Shenyang, China, 9-11 June 2018. IEEE.

[84] Florin Stoican, Ionela Prodan, Dan Popescu, and Loretta Ichim. Constrained trajectory generation for uav systems using a b-spline parametrization. In 2017 25th Mediterranean Conference on Control and Automation (MED), pages 613-618, Valletta, Malta, 3-6 July 2017. IEEE. 
[85] Efstathios Bakolas, Yiming Zhao, and Panagiotis Tsiotras. Initial guess generation for aircraft landing trajectory optimization. In AIAA Guidance, Navigation, and Control Conference, Portland, Oregon, 8-11 August 2011. AIAA.

[86] Wolfgang Honig, James A. Preiss, T.K. Satish Kumar, Gaurav S. Sukhatme, and Nora Ayanian. Trajectory planning for quadrotors swarms. IEEE TRANSACTIONS ON ROBOTICS, 34(4):856-869, August 2018.

[87] Michael A. Feldman. Efficient low-speed flight in a wind field. Master thesis, Virginia Polytechnic Institute and State University, Blacksburg, Virginia, July 1996.

[88] Marcello R. Napolitano. Aircraft Dynamics: From Modeling to Simulation. John Wiley and Sons, Inc., 2012, ISBN: 978-1-118-21492-3.

[89] Jane-Wit Kampoon. WIND FIELD ESTIMATION AND ITS UTILIZATION IN TRAJECTORY AND INPUT PREDICTION. PhD thesis, The University of Texas at Arlington, December 2014.

[90] Mark Peters and Michael A. Konyak. The engineering analysis and design of the aircraft dynamics model for the faa target generation facility. Report, Air Traffic Engineering Co., LLC, 1027 Route 9 South, Palermo, NJ 08223, October 2012.

[91] Min Prasad Adhikari. Real-time autonomous collision avoidance for unmanned aerial vehicles. Master's thesis, Ryerson University, Toronto, Canada, June 2015.

[92] Barnes W. McCormick. Aerodynamics, Aeronautics, And Flight Mechanics. John Wiley and Sons, Inc., 1995.

[93] G.K. Ananda, P.P. Sukumar, and M.S. Selig. Measured aerodynamic characteristics of wings at low reynolds numbers. Aerospace Science and Technology, 42:392-406, April=May 2015.

[94] Diego Pardo, Lukas Moller, Michael Neunert, Alexander W. Winkler, and Jonas Buchli. Evaluating direct transcription and nonlinear optimization methods for robot motion planning. IEEE ROBOTICS AND AUTOMATION LETTERS, 1(2):946-953, July 2016. 
[95] Accessed: July 13, 2019, RASPBERRY PI 3 Model B, Raspberrypi.org, [https://www.raspberrypi.org/products/raspberry-pi-3-model-b/] 2019.

[96] Shahaboddin Owlia. Real-time autonomous obstacle avoidance for low-altitude fixed-wing aircraft. Master thesis, Carleton University, Ottawa, January 2013.

[97] Wei Ren and Ella Atkins. Nonlinear trajectory tracking for fixed wing uavs via backstepping and parameter adaptation. In AIAA Guidance, Navigation, and Control Conference and Exhibit, San Francisco, California, 15-18, August 2005.

[98] Liang Sun. Dynamic Modeling, Trajectory Generation and Tracking for Towed Cable Systems. PhD thesis, Brigham Young University - Provo, Utah, United States, December 2012.

[99] Francisco Gavilán Alfonso Valenzuela, Damián Rivas and Rafael Vázquez. Optimal trajectory generation for uavs using dynamic trajectory modeling. In Proc. of the 1st Workshop on Research, Development and Education on Unmanned Aerial Systems (RED-UAS 2011), pages 29-40, Seville, Spain, November 30th -December 1st 2011.

[100] James Keller and Vijay Kumar. Time-optimal uav trajectory planning for 3d urban structure coverage. In 2008 IEEE/RSJ International Conference on Intelligent Robots and Systems, pages 2750-2757, Nice, France, 22-26 Sept 2008. IEEE.

[101] You Dong Il, Jung Yeon Deuk, Cho Sung Wook, Shin Hee Min, Lee Sang Hyup, and David Hyunchul Shim. A guidance and control law design for precision automatic take-off and landing of fixed-wing uavs. In AIAA Guidance, Navigation, and Control Conference, Minneapolis, Minnesota, 13-16 August 2012. AIAA.

[102] Randal W. Beard, Derek Kingston, Morgan Quigley, Deryl Snyder, Reed Christiansen, Walt Johnson, Timothy McLain, and Michael Goodrich. Autonomous vehicle technologies for small fixed wing uavs. Journal of Aerospace Computing, Information and Communication, 2(1):92-108, January 2005.

[103] Jian Yang, Zhihua Qu, Jing Wang, and Richard A. Hull. Real-time optimized trajectory planning for a fixed-wing vehicle flying in a dynamic environment. JOURNAL OF AEROSPACE ENGINEERING, 22(4):331-341, October 2009. 
[104] Yiming Zhao and Panagiotis Tsiotras. Time-optimal parameterization of geometric paths for fixed-wing aircraft. In AIAA Infotech@Aerospace 2010, Atlanta, Georgia, 20-22 April 2010. AIAA.

[105] Yiming Zhao and Panagiotis Tsiotras. Time-optimal path following for fixed-wing aircraft. Journal of Guidance Control Dynamics, 36(1):83-95, January-February 2013.

[106] Reza Jamilnia and Abolghasem Naghash. Optimal guidance based on receding horizon control and online trajectory optimization. JOURNAL OF AEROSPACE ENGINEERING, 26(4):786-793, October 2013.

[107] Yao Zhao, Yongzhi Sheng, Xiangdong Liu, and Zhen Li. Analytic approach and landing guidance through a novel time-varying sliding mode control method. JOURNAL OF AEROSPACE ENGINEERING, 29(4), 2016.

[108] R. Zardashti, M. J. Yazdanpanah, and A. A. Nikkhah. Nonlinear multiobjective time-dependent $\mathrm{tf} / \mathrm{ta}$ trajectory planning using a network flow-based algorithm. JOURNAL OF AEROSPACE ENGINEERING, 29(2), 2016.

[109] Sheng Zhang, Michael I. Friswell, David J. Wagg, and Guo-Jin Tang. Rapid path planning for zero-propellant maneuvers. JOURNAL OF AEROSPACE ENGINEERING, 29(3), 2016.

[110] Ulrich Kiffmeier. Real-time implementation. CONTROL SYSTEMS, ROBOTICS, AND AUTOMATION, System Analysis and Control: Classical Approaches II, II:237-269, 2009.

[111] Alexandra Grancharova and Tor Arne Johansen. Explicit Nonlinear Model Predictive Control, Theory and Applications. Springer, Verlag Berlin Heidelberg, 2012. ISBN 978-3-642-28780-0.

[112] Bernard Etkin and Lloyd Duff Reid. Dynamics of Flight: Stability and Control. Wiley, 1995.

[113] Marcus Reble. Model Predictive Control for Nonlinear Continuous-Time Systems with and without Time-Delays. PhD thesis, Institut für Systemtheorie und Regelungstechnik, Universität Stuttgart, Stuttgart, February 2013. 
[114] Alexander Joos. Real-Time Predictive Motion Planning for Fixed-Wing Aerial Vehicles. Phd thesis, Universität Stuttgart, February 2014.

[115] Yang Wang and Stephen Boyd. Fast model predictive control using online optimization. IEEE TRANSACTIONS ON CONTROL SYSTEMS TECHNOLOGY, 18(2):267-278, March 2010.

[116] D. Lam, C. Manzie, and M. Good. Model predictive contouring control. In 49th IEEE Conference on Decision and Control (CDC), pages 6137-6142, Atlanta, GA, USA, 15-17 December 2010. IEEE.

[117] Zheng Zhang, Dong Un An, Hyongsuk Kim, and Kil To Chong. Comparative study of matrix exponential and taylor series discretization method for nonlinear odes. Simulation Modelling Practice and Theory, ELSEVIER, 17:471-484, February 2009 .

[118] Z. Zuo. Trajectory tracking control design with command-filtered compensation for a quadrotor. IET Control Theory and Applications, 4(11):2343-2355, 2010.

[119] Teppo Luukkonen. Modelling and control of quadcopter. Independent research project in applied mathematics, Aalto University- School of Science, Espoo, August 222011.

[120] P. Wang, Z. Man, Z. Cao, J. Zheng, and Y. Zhao. Dynamics modelling and linear control of quadcopter. In 2016 International Conference on Advanced Mechatronic Systems (ICAMechS), pages 498-503, Melbourne, Australia, November 30 -December 32016.

[121] Daniel Morgan, Soon-Jo Chung, and Fred Y. Hadaegh. Swarm assignment and trajectory optimization using variable-swarm, distributed auction assignment and model predictive control. In AIAA Guidance, Navigation and Control Conference, Advances in Guidance, Navigation, and Control of Multi-Agent Autonomous Systems, Kissimmee, Florida, 5-9 January 2015. AIAA. 